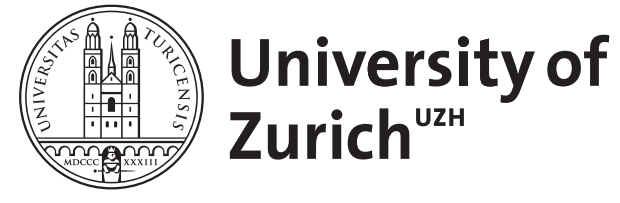

Zurich Open Repository and Archive

University of Zurich

University Library

Strickhofstrasse 39

CH-8057 Zurich

www.zora.uzh.ch

Year: 1986

\title{
Sleep homeostasis
}

Borbély, Alexander A

DOI: https://doi.org/10.1017/s0140525x00046239

Posted at the Zurich Open Repository and Archive, University of Zurich

ZORA URL: https://doi.org/10.5167/uzh-153993

Journal Article

Published Version

Originally published at:

Borbély, Alexander A (1986). Sleep homeostasis. Behavioral and Brain Sciences, 9(3):401.

DOI: https://doi.org/10.1017/s0140525x00046239 


\title{
Evolving concepts of sleep cycle generation: From brain centers to neuronal populations
}

\author{
J. A. Hobson, R. Lydic, and H. A. Baghdoyan \\ Laboratory of Neurophysiology, Harvard Medical School, Boston, Mass. \\ 02115
}

\begin{abstract}
As neurophysiological investigations of sleep cycle control have provided an increasingly detailed picture of events at the cellular level, the concept that the sleep cycle is generated by the interaction of multiple, anatomically distributed sets of neurons has gradually replaced the hypothesis that sleep is generated by a single, highly localized neuronal oscillator.

Cell groups that discharge during rapid-eye-movement (REM) sleep (REM-on) and neurons that slow or cease firing during REM sleep (REM-off) have long been thought to comprise at least two neurochemically distinct populations. The fact that putatively cholinoceptive and/or cholinergic (REM-on) and putatively aminergic (REM-off) cell populations discharge reciprocally over the sleep cycle suggests a causal interdependence.

In some brain stem areas these cell groups are not anatomically segregated and may instead be neurochemically mixed (interpenetrated). This finding raises important theoretical and practical issues not anticipated in the original reciprocal-interaction model. The electrophysiological evidence concerning the REM-on and REM-off cell groups suggests a gradient of sleep-dependent membrane excitability changes that may be a function of the connectivity strength within an anatomically distributed neuronal network. The connectivity strength may be influenced by the degree of neurochemical interpenetration between the REM-on and REM-off cells. Recognition of these complexities forces us to revise the reciprocal-interaction model and to seek new methods to test its tenets.

Cholinergic microinjection experiments indicate that some populations of REM-on cells can execute specific portions of the REM sleep syndrome or block the generation of REM sleep. This observation suggests that the order of activation within the anatomically distributed generator populations may be critical in determining behavioral outcome. Support for the cholinergic tenets of the reciprocal-interaction model has been reinforced by observations from sleep-disorders medicine.

Specific predictions of the reciprocal-interaction model and suggestions for testing these predictions are enumerated for future experimental programs that aim to understand the cellular and molecular basis of the mammalian sleep cycle.
\end{abstract}

Keywords: brain stem; cholinergic systems; monoamines; neuronal populations; oscillators; sleep; sleep disorders; ultradian rhythms

\section{From brain centers to neuronal populations: A scientific paradigm shift?}

Mammalian neurobiology seeks to identify the neurons that mediate behavioral acts and states and to specify the mechanisms by which the critical neuronal ensembles are activated and inactivated. Most studies of the neural control of behavior have focused upon specifiable motor acts (e.g., limb flexion or extension) and have ignored the sets of background conditions or behavioral states (e.g., sleep, wakefulness, or anesthesia) out of which the acts arise. The investigation of motor acts can offer the relative advantage of the reflex paradigm. For example, cellular studies of segmental reflex control can often be localized to restricted neuronal circuitry. By contrast, physiological studies of behavioral states such as sleep must contend with endogenous changes occurring simultaneously in a multiplicity of diffuse neuronal circuits. Hobson (1978) has discussed the evolution of the state concept and defined a behavioral state operationally as a set of numerical values that its component variables may be assigned at a given point in time.
Only since the 1950s has it been recognized that sleep is a heterogeneous behavioral state. Mammalian sleep is a bistable process consisting of continuous alterations between two different states. Information about the nomenclature (Czeisler, Borbely, Hume, Kobayashi, Kronaure, Schulz, Weitzman, Zimmerman \& Zulley 1980; Roffwarg 1979; Ursin \& Sterman 1981) and the temporal organization of sleep states has been presented in detail elsewhere (Kraemer, Hole \& Anders 1984; Lavie \& Kripke 1981; Schulz, Dirlich, Balteskonis \& Zulley 1980). Briefly, the two distinct sleep states have been given a variety of names, which are usually descriptors of bioelectric recordings used to characterize these two behavioral states. For example, one state of sleep is characterized by rapid eye movements (REM) but is also referred to as desynchronized, paradoxical, or active sleep. The other state of sleep is characterized by a synchronized electroencephalogram and is referred to as synchronized, slow-wave, quiet, or non-REM (NREM) sleep. The REM-and-NREM nomenclature will be used throughout this paper.

The important point, from a broader neurobiological 
perspective, is that REM sleep occurs as an endogenously generated rhythm in all terrestrial, placental mammals, including man (Tobler 1984). The REM-and-NREM sleep cycle exhibits species-specific period lengths that are always in the ultradian (much less than $24 \mathrm{hrs}$ ) range (Campbell \& Tobler 1984). Thus, these cyclic alterations in behavioral state represent a fundamental biological rhythm.

Until recently, the states of waking and sleep were studied within the paradigm of research on neural centers. The neural-centers concept is a special case of the cerebral-localizationist trend that dominated the physiological study of behavior in the 1930s and 1940s. Early exponents of the neural-centers concept were Hess (1931), who postulated waking and sleep centers in the diencephalon, and Bremer (1935), who first suggested that waking and sleep were regulated intrinsically, perhaps by the brain stem. The subsequent studies of arousal by Moruzzi and Magoun (1949), of NREM sleep by Moruzzi (1960), and of REM sleep by Jouvet (1962) all postulated specific brain regions or even particular nuclei involved in behavioral-state control. These hypotheses were heuristically useful because they focused attention on the physiological mechanisms underlying behavioral states. Such hypotheses were practically limited, however, since the lesion and electrical-stimulation techniques were incapable of determining the precise cellular and molecular mechanisms involved in triggering and maintaining behavioral states.

Gradually replacing the concept of discrete regulatory centers controlling sleep and wakefulness is the emerging concept of dynamic interaction between anatomically distributed neuronal populations. The notion of functionally distinct neuronal populations was foreshadowed by Hess's (1931) concept of trophotrophic (cholinergic) and ergotrophic (aminergic) centers in the hypothalamus. Applied to behavioral-state control, this idea was further concretized and extended in Jouvet's (1962) work on the pontine mechanisms of REM sleep, which first implicated a cholinergic trigger mechanism. Jouvet later incorporated the histofluorescent anatomical data of the Swedish school (Dahlstrom \& Fuxe 1965) when he suggested that each specific state was controlled by a specific aminergic cell group (Jouvet 1969). Since many of the chemically specific neurons were found in extended sets of brain stem nuclei (Morgane \& Stern 1974), the idea of an anatomically distributed system was thus clearly implied even in Jouvet's highly specified formulations of sleep cycle control.

The distributed-neuronal-population concept has also been reinforced by more recent evidence obtained when the electrical activity of individual neurons was recorded from behaving animals. The contrast between the braincenters concept and the neuronal-populations concept becomes particularly sharp when distinctive single-cell firing patterns are displayed by neurons concentrated in certain brain regions or localized in scattered, but chemically distinct, brain nuclei. Groups of cells may attract attention because of their unique behavioral-statespecific discharge patterns. This is what has been found in recent research on the brain stem mechanisms controlling the behavioral states of waking and sleep. For example, during REM sleep some neurons discharge more
(REM-on cells), and some neurons cease discharging (REM-off cells). (See Figure 2 caption for a quantitative definition of REM-on and REM-off cells.) The REM-on and REM-off discharge patterns will be discussed in detail to illustrate the interpretative and methodological problems of sleep neurobiology. Throughout this discussion we will distinguish between generator and modulator populations. Generator populations include neurons that may act as initiators or effectors of a state or the physiological or behavioral components of the state. Modulator neurons alter the postsynaptic response of a cell to some parallel presynaptic input. In so doing, they may alter the mode of neuronal operation, including the output of generator populations, thereby determining state properties.

It is the purpose of this paper to trace the conceptual and theoretical evolution of sleep neurophysiology in its transition from the neuronal-centers to the neuronalpopulations paradigm. In doing so, the present account will focus on evidence regarding the first cellular and mathematical model of mammalian behavioral-state control, that of reciprocal interaction between putatively cholinergic and aminergic brain stem neurons, which appeared 10 years ago (Hobson, McCarley \& Wyzinski 1975; McCarley \& Hobson 1975b). In the subsequent decade, the initial finding and concepts have stimulated discussion and controversy. The central goal of the present paper is to move beyond the debate about the significance of correlations (between cellular discharge patterns and sleep states) and to stimulate a new era of causal hypothesis testing by reformulating the reciprocalinteraction hypothesis in terms more amenable to unambiguous empirical evaluation.

\section{A. General features of the reciprocal-interaction model of sleep cycle control}

We believe it essential to preface our consideration of the relationship between empirical data and conceptual models with some discussion of the nature and utility of models in general and of the reciprocal-interaction concept in particular. Models are useful and perhaps even essential in the conceptual organization of data at critical points in the growth of science. With the growth in its data base, neurobiology has developed models of increasing complexity. For example, the neuron doctrine of Ramón y Cajal might be described as the fundamental first-order or structural model of modern neurobiology. In his sketches of linear neuronal interactions, Cajal anticipated Sherrington's second-order or dynamic-reflex model. The reflex concept made possible the detailed analysis of individual sensorimotor systems. Sherrington's coworker, Graham-Brown, added the neuronaloscillator concept to the dynamic-reflex model. This extension was based on Graham-Brown's (1914) observation that neuronal activity was not only responsive to external stimuli but also spontaneous, dynamic, and intrinsically periodic (Gallistel 1980).

The reciprocal-interaction model of sleep cycle control tries to account for neuronal discharge activity, which is characteristically spontaneous, continuous, and periodic. These formal properties of neuronal activity are precisely those which are likely to be of more fundamental rele- 
vance to the still-higher-order concept of behavioral state control. One way to make the conceptual transition from the reflex paradigm to the behavioral-state paradigm is to view a given behavioral state (e.g., REM sleep) as a constellation of temporally coordinated physiological traits, each of which manifests the reflexivity of individual sensorimotor systems.

While model building is essential for integrating facts, making predictions, and testing hypotheses, we also recognize two pitfalls in the use of any model. One is reification, which occurs when the abstract model (a hypothetical construct) is confused with the reality of the physiological system being modeled. The other pitfall is the untestability of a model so flexible that it can be bent to suit any datum. In offering this revision of the reciprocal-interaction model for open peer commentary, we appeal to our colleagues for help in avoiding either of these pitfalls.

In constructing this revision we have tried to clarify the present nature, value, and limitations of the reciprocalinteraction model. Because this model superficially resembles the Sherringtonian reflex model it has sometimes mistakenly been construed as positing a mere variant of simple reciprocal innervation (as in the crossedextension reflex). It is important to note that such a reading involves both a lexical mistake and a misunderstanding since the reciprocal-interaction model assumes - and begins to account for - the temporal organization of spontaneous fluctuations in the excitability levels of neurons that may be organized reflexively, or nonreflexively, throughout the brain. In contrast to the reflex concept, the reciprocal-interaction model of behavioral-state control is thus neither restricted to local circuits nor inputdependent. A patient and careful evaluation of these revised concepts can productively contribute to the continued development of sleep research. We therefore appeal to our readers - especially those serving as commentators - for a detailed assessment of the weaknesses as well as the strengths of these concepts. We believe that only through such a properly balanced evaluation can our dialogue contribute fresh perspectives and avoid sterile debate.

\section{B. Heuristic value of the reciprocal-interaction model: When should a model be altered and when should it be abandoned?}

When a model can be altered by a simple modification of its assumptions (without violating its essential or fundamental structure) it should be so altered and retained. When a model is demonstrated to be fundamentally wrong, or when it fails to generate testable hypotheses, it should be abandoned. As we hope to make clear, the testing of hypotheses derived from the reciprocal-interaction model has only begun, and the initial results strongly encourage further work.

A critical question about the usefulness of the model is: Can the reciprocal-interaction model be proved wrong? In other words, is it, in Popper's (1962; 1974) terms, a genuinely scientific hypothesis? The answer to this question is yes, as will be shown in Part V of this paper, where testable predictions are enumerated that could further confirm or unequivocally refute the model.

\section{The problem of correlation versus causation}

One problem fundamental to behavioral neurobiology, and indeed to all studies of regulatory physiology, is the distinction between correlation and causation. We believe that this conceptual issue is at the root of the interpretative differences that have divided investigators in the field of sleep neurophysiology.

The problem concerns the adequacy of evidence from single-cell recording or central-nervous-system (CNS) lesions as a basis for accepting or rejecting the hypothesis that a given brain region plays a causal role in generating a behavioral or physiological event. Data on behavioral state control will be discussed in detail using the concepts and interpretive logic that follow.

For cellular discharge data:

1. A strong positive or negative correlation between single-cell firing rate and a behavioral state is compatible with a hypothesis of cause but cannot be taken as more than suggestive evidence that the cellular activity causes the behavioral state. An example would be the high discharge rate of pyramidal tract motoneurons during REM sleep (Evarts 1964), a finding that, in and of itself, cannot be distinguished from similar results at the level of the brain stem (Steriade \& Hobson 1976). Which is cause, which is effect, and which a mere correlate?

2. A corollary of the first principle concerns the fact that most brain regions and their component nuclear groups have multiple regulatory roles. An example would be the putative role of serotonergic neurons in pain modulation, temperature control, and sleep regulation. It would thus be inappropriate to conclude that because such neurons mediate pain and temperature control they cannot mediate sleep. For example, serotonergic neurons cease firing during REM sleep (McGinty \& Harper 1976), temperature control is lost in REM sleep (Parmeggiani 1981), and pain sensation is rare in dream reports (McCarley \& Hoffman 1981). For any neurophysiological theory of sleep to be adequate it should aim to integrate observations from multiple physiological systems (Figure 1; Table 1).

3. Since neuronal populations commonly subserve many regulatory functions, and since the CNS exhibits considerable functional plasticity after experimental lesions, the relationships between neuronal discharge and a given behavioral state may not be immutable. After surgical or pharmacological manipulations, neuronal firing patterns may even be dissociated from a given behavioral state. An example of altered phase dependence would be the state-independent pontogeniculooccipital (PGO) waves that are produced after brain amine depletion with parachlorophenylalanine (Jouvet 1972) or with localized administration of cholinergic agonists (Vivaldi, McCarley \& Hobson 1980).

For data obtained from experimental lesions:

1. The loss of a behavioral state after a CNS lesion is compatible with the idea that the brain region may be involved in generating that behavioral state, but such evidence is no more than suggestive and may even be quite misleading. Numerous examples are discussed in Section A, Part II; the complexity of this point is also illustrated by the cessation of respiration or loss of sleep seen after midline knife cuts in the brain stem (Mancia 
1969). Should Mancia's finding be attributed to destruction of cell bodies (e.g., raphe neurons) or to destruction of decussating fibers? And would such fibers be of local or remote origin?

2. Persistence of a behavioral state following a lesion indicates only that the destroyed CNS structure is not necessary for the expression of the behavioral state; it does not prove that the brain region in question normally plays no physiological role in the generation of that behavioral state. The redundancy that is typical of most CNS control systems may explain, for example, the fact that animals with visual cortex ablations can still "see" with their colliculi (Sprague 1966) or that monkeys can still move after destruction of the pyramidal tract (Bucy, Ladpli \& Erlich 1966). One would not conclude from these data that the cortex has no role in vision or that the pyramidal tract has no role in movement (see also Campion, Latto \& Smith 1983).

The general conclusion emerging from single-cell recording and experimental lesion studies is that neither method, either alone or in combination with the other, provides evidence adequate for accepting or rejecting any hypothesis of causality with complete confidence. Given these severe methodological limitations, it is important to be both cautious in interpreting data and imaginative in developing additional ways to test hypotheses that a neuronal population causes a particular stage of sleep. The difficulties in obtaining definitive evidence for or against such causal hypotheses in the case of sleep cycle control will be discussed to illustrate (1) the strengths and problems of the neuronal-population approach and (2) the need for developing specific criteria with which to evaluate the hypothesis that a cellular population causes a behavioral state.

\section{A neuronal-population concept of behavioral-state control: Evolution of the reciprocal-interaction model}

Over the past decade, the initial formulation of the reciprocal-interaction model of mammalian sleep cycle control (Hobson et al. 1975; McCarley \& Hobson 1975b) has been revised in accordance with new evidence from single-unit recording and chemical microinjection experiments in animals and from human clinical studies (Hobson 1984; McCarley 1978; 1980; McCarley \& Massaquoi 1985). In its original form, the reciprocal-interaction model postulated that the behavioral states constituting the mammalian sleep cycle were a function of the out-ofphase discharge profiles displayed by two brain stem neuronal populations. The REM-on cells were first found in the gigantocellular tegmental field (FTG) of the pontine reticular formation and were postulated to play a cholinergically mediated role in generating REM sleep. The REM-off cells were first found in the locus coeruleus (LC) and were postulated to play a noradrenergic, permissive role by disinhibiting the REM-on cells. The model, which aimed to be synaptically explicit and mathematically precise in its attempt to account for the cycling of NREM and REM sleep, could also be extended to account for waking state features in terms of high levels of aminergic neuronal activity.

Replacing these original postulates of a REM sleep generator zone highly localized to the pontine FTG is the present concept of an anatomically distributed population of neurons (REM-on cells) in the brain stem whose activation is postulated to play a key role in generating REM sleep. Figure 1 schematizes the anatomically distributed nature of some of the neuronal populations studied in relation to behavioral-state control.

A note regarding the anatomical nomenclature in Figure 1 and throughout the text: In constructing this revision of the reciprocal-interaction model, we have continued to use the terminology of Berman (1968). Berman's stereotaxic atlas is a standard reference found in virtually every neurophysiology laboratory where the domestic cat is the animal studied. We appreciate the cytological heterogeneity of the brain stem reticular formation, and when we refer to the FTG of Berman (1968), unless otherwise stated, we are emphasizing that portion of the FTG defined by Brodal (1969) as the medial pontine reticular formation. The general features of the figure include:

1. The putatively cholinergic/cholinoceptive REM-on cell network is widespread, and its component neurons at the cortical, brain stem, and spinal cord levels are postulated to interact with motor-control systems during wakefulness, as well as during REM sleep.

2. The excitatory interconnections of the REM-on cell network are such that the REM-on activation process could theoretically start from several places within the network if the inhibitory aminergic input were removed uniformly. Once the REM-on discharge is started it is postulated to increase exponentially to some maximum level, reinforced by the excitatory synaptic interconnections.

3. Figure 1 distinguishes between two critically selective neuronal populations: (a) the aminergic (REM-off) cells, which are postulated to exert an inhibitory restrain during waking so as to release the REM-on neurons progressively to the point of autoactivation during REM sleep, and (b) the inhibitory pontomedullary neurons, which are postulated to actively block the somatic motor output during REM sleep.

4. The interaction between the REM-off (putative modulatory) and the REM-on (putative generator) networks is also anatomically widespread throughout the CNS (for details see Table 1B). Based on this diffuse anatomical distribution, any localization of a putative REM sleep generator even to the brain stem could, theoretically, be an illusion. Our reasoning in making this suggestion is the following:

(a) The concentration in the brain stem of aminergic cell bodies (see again Figure 1; and for details, Table 1B) would indicate that the aminergic portion of the putative REM sleep oscillator is localizable to the brain stem. The widespread distribution of the REM sleep generator population (Figure 1) suggests that the other, putatively cholinoceptive/cholinergic side of the proposed oscillator is much less likely to be so localized. The experimental evidence supporting these ideas is discussed in detail in Part IV and further illustrated in Figures 2 and 4.

(b) The putative effectors of some REM sleep phenomena (Figure 1) are localizable to the brain stem (e.g., those neurons generating rapid eye movements, PGO waves, and facial muscle twitches), but other effectors of REM sleep are not in the brain stem (e.g., the effector 


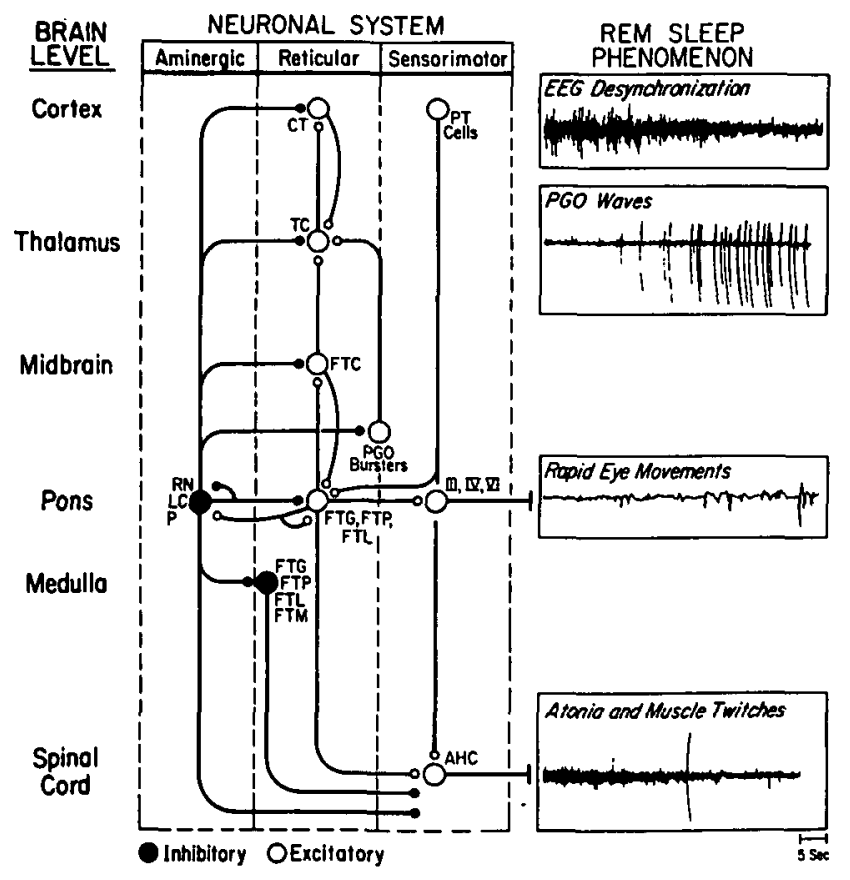

Figure 1. Schematic representation of the REM sleep generation process. A distributed network involves cells at many brain levels (left). The network is represented as comprising three neuronal systems (center) that mediate REM sleep electrographic phenomena (right). Postulated inhibitory connections are solid circles; postulated excitatory connections are open circles. In this diagram, no distinction is made between neurotransmission and neuromodulatory functions of the depicted neurons. Table $1 \mathrm{~A}$ gives a selective outline of the physiological evidence supporting this schematization, and the anatomical evidence on which the synaptic connections are based is outlined in Table 1B. We caution the reader that the precise synaptic signs of many of the aminergic and reticular pathways remain to be demonstrated. This is particularly true with respect to the possible state dependency of the postulated synaptic signs. In many cases, the neuronal architecture is also known to be far more complex than we have indicated (e.g., the thalamus and cortex).

There are postulated to be two additive effects of the marked diminution in firing by aminergic ncurons at REM sleep onset: disinhibition (through removal of negative restraint) and facilitation (through positive feedback). The net result is strong tonic and phasic activation of reticular and sensorimotor neurons.

REM sleep phenomena are postulated to be mediated as follows:

EEG desynchronization results from a net tonic increase in reticular, thalamocortical, and cortical neuronal firing.

PGO waves are the result of tonic disinhibition and phasic excitation of burst cells in the lateral pontomesencephalic tegmentum. (Note that no excitatory input is shown because none has yet been demonstrated.)

The rapid eye movements are the consequence of phasic firing by reticular and vestibular cells; the latter (not shown) directly excite the oculomotor neurons.

Muscular atonia is the consequence of tonic postsynaptic inhibition of the anterior horn cells by the pontomedullary reticular formation.

Muscle twitches occur when excitation by reticular and pyramidal tract motoneurons phasically overcomes the tonic inhibition of the anterior horn cells.

Anatomical abbreviations: $\mathrm{RN}=$ raphe nuclei; $\mathrm{LC}=$ locus coeruleus; $\mathrm{P}=$ peribrachial region; $\mathrm{FTG}=$ gigantocellular teginental field; FTC = central tegmental field; FTP = parvocellular tegmental field; FTM = magnocellular tegmental field; $\mathrm{TC}=$ thalamocortical; $\mathrm{CT}=$ cortical; $\mathrm{PT}$ cell $=$ pyramidal
Hobson, Lydic \& Baghdoyan: Sleep cycle generation

neurons of EEG desynchronization and alpha motoneurons effecting somatic muscular atonia).

(c) According to this concept, one could anticipate that extensive destruction of REM-on cells in the brain stem might be without effect on some of the tonic manifestations of REM sleep which are derived from extra-brainstem effectors.

(d) A key remaining question concerns the degree of dissociation of the physiological signs of REM sleep that could be effected by neuronal destruction. Part IV of this paper describes the dissociations of the physiological signs of REM sleep that can be systematically produced using chemical microinjection techniques.

The most essential neurophysiological difference between the waking and the REM sleep state now appears to be the level of discharge activity in the subpopulation of putatively modulatory REM-off cells located in several aminergic nuclei throughout the pontomesencephalic brain stem. These aminergic nuclei are widespread in the pontine tegmentum, display progressive decreases in firing rate during REM sleep, and may disinhibit the anatomically distributed REM-on neurons (Table 1A). Like the REM-on populations, these level-setting or modulatory REM-off populations are more widely distributed than was first supposed. They are currently known to include at least two chemically distinct groups: the noradrenergic and the serotonergic subpopulations; and they, are found to be concentrated in at least three brain stem regions: the raphe nuclei, the locus coeruleus, and the peribrachial region. Many brain stem nuclei contain cells of both the REM-on and the REM-off type, thus indicating that the REM sleep oscillator network is not only more widely distributed than previously believed but also neurochemically interpenetrated.

\section{A. The putative generator population}

1. Identity and characteristics of the REM-on cells. During REM sleep, most of the neurons recorded throughout the brain have been shown to be activated to levels at least as high as those seen in the waking state (Steriade \& Hobson 1976). The most conspicuous exception is the population of REM-off cells that will be discussed below (Part II, Section B) with respect to their proposed modulatory or level-setting interaction with the REM-on population.

Since so many neurons are of the REM-on type, it is logical to propose that a vast ensemble of cells constitutes the population that finally acts as effectors of the physiological signs of REM sleep, such as cortical desynchronization, muscular atonia, or the rapid eye movements themselves. A putative neuronal trigger zone that initiates the recruitment process leading to the coordinated physiological manifestations of REM sleep remains to be unequivocally identified.

The neuronal mechanisms to be accounted for in REM sleep generation ultimately include tonic and phasic excitation of sensory and motor neurons at many levels of the nervous system during sleep. From the tentative

cell; III = oculomotor; IV = trochlear; $\mathrm{V}=$ tirgeminal motor nuclei; $\mathrm{AHC}=$ anterior horn cell.

The time calibration for the four simultaneously recorded polygraphic records is 5 seconds. 
Hobson, Lydic \& Baghdoyan: Sleep cycle generation

Table 1A. Electrophysiological studies of the REM sleep generation process

\begin{tabular}{|c|c|c|}
\hline Brain Level & Reference & Conclusion \\
\hline Cortex & $\begin{array}{l}\text { Evarts } 1960 \\
\text { Evarts } 1964 ; 1965 \\
\text { Steriade \& Deschenes } 1974 \\
\text { Steriade et al. } 1974 \\
\text { Steriade et al. } 1978 \\
\text { Steriade et al. } 1979\end{array}$ & $\begin{array}{l}\text { Neurons in lateral gyrus of visual cortex are active in REM sleep. } \\
\text { Pyramidal tract neurons are active in REM sleep. } \\
\text { Pyramidal tract neurons and interneurons discharge at high levels in REM sleep. } \\
\text { Pyramidal cells and putative interneurons in cortical areas } 5 \text { and } 7 \text { discharge at high levels during REM sleep. } \\
\text { Neurons of parietal association cortex revealed similar processes of excitation and inhibition during W and REM } \\
\text { sleep. }\end{array}$ \\
\hline Thalamus & $\begin{array}{l}\text { Glenn et al. } 1982 \\
\text { Bizzi } 1966 \\
\text { Sakakura } 1968 \\
\text { Steriade et al. } 1969 \\
\text { Hirsch et al. } 1983\end{array}$ & $\begin{array}{l}\text { Ventromedial nucleus of thalamus provides depolarizing projections to layer J of anterior neocortex. } \\
\text { Single cells in lateral geniculate nucleus (LGN) fire in phase with PGO waves. } \\
\text { Discharge of LGN neurons is correlated with positive phase of PGO waves in REM sleep. } \\
\text { Responsiveness of thalamic ventrolateral nucleus to electrical stimulation is highest in REM sleep. } \\
\text { Intracellular recording of LGN neurons during REM sleep reveals depolarization. }\end{array}$ \\
\hline Midbrain & $\begin{array}{l}\text { Huttenlocher } 1961 \\
\text { Kasamatsu } 1970 \\
\text { Steriade \& Glenn } 1982 \\
\text { Steriade et al. } 1982\end{array}$ & $\begin{array}{l}\text { Mesencephalic reticular formation (MRF) units have greatest activity in REM sleep. } \\
\text { MRF projections excite intralaminar thalamic neurons projecting to the cortex. } \\
\text { MRF discharge contributes to thalamocortical activation leading to EEG desynchrony in W and REM sleep. }\end{array}$ \\
\hline Pons & $\begin{array}{l}\text { Chu \& Bloom 1974a } \\
\text { Aston-Jones \& Bloom } 1981 \\
\text { Foote et al. 1983 } \\
\text { McGinty \& Harper } 1976 \\
\text { Saito et al. 1977 } \\
\text { McCarley et al. } 1978 \\
\text { Mergner \& Pompeiano } 1978 \\
\text { Cespuglio et al. } 1981 \\
\text { Heym et al. 1982 } \\
\text { Hobson, McCarley et al. } \\
\text { 1983 } \\
\text { Lydic et al. 1984 } \\
\text { McCarley \& Ito } 1983 \\
\text { Ito \& McCarley } 1984\end{array}$ & $\begin{array}{l}\text { Dorsal raphe neurons have lowest discharge rate in REM sleep (REM-of). } \\
\text { Peribrachial pontine tegmental neurons have REM-off discharge. } \\
\text { Phasic increase in FTG cellular discharge phase leads PGO waves of REM sleep by } 900 \mathrm{~ms} . \\
\text { Vestibular neuronal firing phase locked to rapid eye movements in REM sleep. } \\
\text { Raphe magnus neurons have REM-off discharge pattern. } \\
\text { Raphe pontis neurons have REM-off discharge pattern. } \\
\text { Nucleus linearis centralis neurons have REM-off discharge pattern. } \\
\text { Dorsal raphe discharge rhythm phase-locked to ultradian sleep cycle. } \\
\text { Intracellular recording of medial pontine reticular formation (mPRF) neurons reveals a phasic depolarization cor- } \\
\text { related with PGO waves. } \\
\text { Intracellular recording of mPRF neurons shows a tonic depolarization of membrane potential prior to and } \\
\text { throughout REM sleep. } \\
\text { Stimulation of FTG evokes monosynaptic EPSPs in contralateral mPRF. }\end{array}$ \\
\hline Medulla & $\begin{array}{l}\text { Orem et al. } 1974 \\
\text { Netick et al. } 1977 \\
\text { Kanamori et al. } 1980 \\
\text { Chase et al. } 1981 \\
\text { Sakai et al. } 1983 \\
\text { Steriade et al. } 1984\end{array}$ & $\begin{array}{l}\text { Medullary respiratory neurons have decreased discharge during REM sleep. } \\
\text { Lateral and gigantocellular tegmental field neurons discharge selectively in REM sleep. } \\
\text { Medullary nucleus reticularis gigantocellularis contains REM-on cells. } \\
\text { Intracellular studies show that neurons of the nucleus reticularis gigantocellularis are depolarized during REM } \\
\text { sleep. } \\
\text { Raphe magnus and raphe pallidus contain REM-off cells. }\end{array}$ \\
\hline Spinal cord & $\begin{array}{l}\text { Gassel et al. } 1964 \\
\text { Kubota \& Kidokoro } 1965 \\
\text { Morrison \& Pompeiano } \\
\text { 1965a; 1965b; 1965c } \\
\text { Glenn \& Dement 198la; } \\
\text { 1981b } \\
\text { Morales \& Chase } 1978 \\
\text { Fung et al. } 1982 \\
\text { Chase \& Morales } 1983 \\
\text { Glenn \& Dement } 1985\end{array}$ & $\begin{array}{l}\text { Stimulation of nucleus reticularis pontis oralis induces a REM-specific, long-latency hyperpolarization in lumbar } \\
\text { motoneurons. } \\
\text { Alpha motoneurons are depolarized during the phasic myoclonic activation of REM sleep. }\end{array}$ \\
\hline
\end{tabular}

Note: This table provides selected references supporting the functional role of the schematized neuronal connections outlined in Figure 1.

scheme drawn in Figure 1 and documented in Tables 1A and $\mathrm{IB}$, it can be seen that the components of this vast network are mutually enhancing; it is therefore difficult to assert with certainty that one or another part of the system plays the role of trigger or prime mover for the excitation process initiating a REM sleep episode. The initial defense of the idea that FTG neurons were the executors of REM sleep helped to perpetuate a controversy which one hopes can be superseded by the more supple account of sleep cycle generation developed here.

Questions concerning the appropriateness of hierarchically arranged control models are not limited to REM-sleep-generating mechanisms. For example, since cortical, rubral, and pontine neurons may all contribute 
Table 1B. Anatomical substrates of REM sleep generation

\begin{tabular}{|c|c|c|}
\hline Brain level & Reference & Conclusion \\
\hline Cortex & $\begin{array}{l}\text { Lund et al. } 1975 \\
\text { Rossi \& Brodal } 1956 \\
\text { Wiesendanger } 1969\end{array}$ & $\begin{array}{l}\text { Neocortex projects to thalamus } \\
\text { PT cells project to pontine RF } \\
\text { PT cells project to spinal cord }\end{array}$ \\
\hline Thalamus & Jones \& Leavitt 1974; Hendry et al. 1979 & Thalamus projects to nencortex \\
\hline Midbrain & $\begin{array}{l}\text { Edwards \& DeOlmos } 1976 \\
\text { Edwards } 1975 \\
\text { Sakai } 1980 \text {; Jones } 1985 ; \text { Sofroniew et al. } 1985\end{array}$ & $\begin{array}{l}\text { Midbrain RF projects to thalamus } \\
\text { Midbrain RF projects to pontine RF } \\
\text { PGO burst cell zone projects to thalamus }\end{array}$ \\
\hline \multicolumn{3}{|l|}{ Pons } \\
\hline Raphe & $\begin{array}{l}\text { Azmitia \& Segal } 1978 \\
\text { Moore et al. } 1978 \\
\text { Sakai, Touret et al. } 1977 \\
\text { Conrad et al. 1974; Sakai, Touret et al. 1977; Gallager \& Pert } 1978 \\
\text { Mosco et al. 1977 } \\
\text { Taber-Pierce et al. 1976; Pickel et al. 1977; Sakai, Touret et al. 1977; Morgane \& Jacobs } 1979 \\
\text { Sakai, Touret et al. 1977 } \\
\text { Yezierski et al. } 1982 \\
\text { Tohyama, Sakai, Touret et al. } 1979 \\
\text { Martin et al. 1978; Bowker et al. } 1983\end{array}$ & $\begin{array}{l}\text { DRN projects to neocortex } \\
\text { DRN projects to thalamus } \\
\text { DRN projects to midbrain } R F \\
\text { DRN projects to pontine RF } \\
\text { DRN projects to DRN } \\
\text { DRN projects to LC } \\
\text { DRN projects to Pbl } \\
\text { DRN projects to medullary RF } \\
\text { DRN projects to spinal cord } \\
\text { NRM projects to spinal cord }\end{array}$ \\
\hline \multicolumn{3}{|l|}{ Pons } \\
\hline LC & $\begin{array}{l}\text { Foote et al. 1983; Jones \& Moore } 1977 \\
\text { Jones et al. } 1977 \\
\text { Jones \& Moore } 1977 \\
\text { Sakai } 1980 \\
\text { Scheibel \& Scheibel 1958; Sladek 1971; Maeda et al. 1973; Chu \& Bloom 1974b; Sakai, } \\
\text { Touret et al. 1977; Sakai et al. 1979 } \\
\text { Fuxe 1965; Chu \& Bloom 1974b; Sakai, Salvert et al. 1977 } \\
\text { Scheibel \& Scheibel 1958; Fuxe 1965; Swanson 1976; Sakai, Touret et al. } 1977 \\
\text { Sakai, Touret et al. 1977 } \\
\text { Tohyama, Sakai, Touret et al. 1979; Foote et al. } 1983\end{array}$ & $\begin{array}{l}\text { LC projects to neocortex } \\
\text { LC projects to thalamus } \\
\text { LC projects to midbrain RF } \\
\text { LC projects to PCO burst cell zone } \\
\text { LC projects to pontine RF } \\
\text { LC projects to DRN } \\
\text { LC projects to LC } \\
\text { LC projects to } \mathrm{Pbl} \\
\text { LC projects to spinal cord }\end{array}$ \\
\hline \multicolumn{3}{|c|}{ - } \\
\hline $\mathrm{Pbl}$ & $\begin{array}{l}\text { Sakai } 1980 \\
\text { Sakai } 1980 \\
\text { Sakai et al. } 1979 \\
\text { Sakai, Salvert et al. } 1977 \\
\text { Sakai, Touret et al. } 1977 \\
\text { Tohyama, Sakai, Touret et al. } 1979\end{array}$ & $\begin{array}{l}\text { Pbl projects to neocortex } \\
\mathrm{Pbl} \text { projects to thalamus } \\
\text { Pbl projects to PGO burst cell zone } \\
\text { Pbl projects to pontine } \mathrm{RF} \\
\mathrm{Pbl} \text { projects to DRN } \\
\mathrm{Pbl} \text { projects to LC } \\
\text { Pbl projects to spinal cord }\end{array}$ \\
\hline \multicolumn{3}{|l|}{ Pons } \\
\hline $\begin{array}{l}\text { Pontine } \\
\mathrm{RF}\end{array}$ & $\begin{array}{l}\text { Buttner-Ennever \& Henn } 1976 \\
\text { Peterson 1980; Pompeiano } 1980 \\
\text { Ramón y Cajal 1952; Scheihel \& Scheibel 1958; Walberg 1974: Graybiel } 1977 \\
\text { Nauta \& Kuypers 1958; Valverdi 1961; Aghajanian \& Wang 1977; Gallager \& Pert } 1978 \\
\text { Nauta \& Kuypers 1958; Scheibel \& Scheibel 1958 } \\
\text { Sakai et al. 1979; Jones 1985a } \\
\text { Tohyama, Sakai, Salvert et al. 1979; Jones 1985; Bowker et al. } 1983\end{array}$ & $\begin{array}{l}\text { Pontine RF projects to midbrain RF } \\
\text { Pontine RF projects to III, IV, VI } \\
\text { Pontine RF projects to pontine RF } \\
\text { Pontine RF projects to DRN } \\
\text { Pontine RF projects to LC } \\
\text { Pontine RF projects to medullary RF } \\
\text { Pontine RF projects to spinal cord }\end{array}$ \\
\hline Medulla & Toyhama, Sakai, Salvert et al. 1979; Sakai et al. 1981; Jones 1985a & Medullary RF projects to spinal cord \\
\hline
\end{tabular}

Note: This table provides selected references supporting the structural basis of the schematic model outlined in Figure 1.

to the phasic excitation of spinal motoneurons, it would be difficult to assign a principal role to any one of them in generating movement; the ensemble works as a whole, and motor action may be initiated at several points in the system.

a. The FTG generator hypothesis. Historically, the initial studies of cellular discharge during REM sleep suggested that the FTG might constitute a trigger zone for the initiation of REM sleep (Hobson, McCarley, Pivik \& Freedman 1974; Hobson, McCarley, Freedman \& Pivik 1974; McCarley \& Hobson 1975a). The hypothesis that the FTG constitutes the REM sleep generator is at the center of the discussion and debate stimulated by the reciprocal-interaction model.
It is now clear that the process of REM sleep generation is far too complex to be accounted for solely by the FTG generator hypothesis. First, it has been shown that the neuronal discharge selectivity of FTG neurons recorded from head-restrained animals is not limited to REM sleep. In freely moving animals these pontine cells, like cells elsewhere throughout the CNS, discharged as intensely in association with particular movements in waking as they did during REM sleep (Siegel \& McGinty 1977; Siegel, McGinty \& Breedlove 1977; Vertes 1977). It has also been shown that kainic acid lesions in the pontine reticular formation that destroy FTG cells do not prevent REM sleep (Drucker-Colin \& Bernal-Pedraza 1983; Sastre, Sakai \& Jouvet 1981). This finding reinforces the 
notion that the REM-on population is anatomically distributed and that only large lesions of what still appears to be the most critical portion for REM sleep, the pontine reticular formation, can eliminate all manifestations of REM sleep (Jones 1979).

The discovery that REM sleep discharge selectivity characterized FTG cells only in head-restrained animals overthrew the original assumption that the FTG population was the sole executor or even an essential generator of REM sleep. This finding also supported the idea that the FTG was only one of a set of upper motor neuronal systems to be activated in REM sleep. The relationship between FTG discharge and certain movements does not refute the more cautious suggestion that activation of the FTG and other motor neuronal systems during REM sleep could nonetheless be involved in the generation of many REM sleep events (e.g., the rapid eye movements themselves, the muscle twitches, and even the PGO waves).

Since all results agree that FTG cells are dramatically activated in REM sleep, numerous studies have indicated the importance of understanding how this activation is produced. Unfortunately, the cellular mechanisms of FTG activation during REM sleep are in no way explained by establishing a correlation between specific movements and waking-state FTG discharge. Hence, it is clearly premature to conclude that FTG activation either is entirely movement-related or has no significance in the normal triggering of REM sleep. Neither of these alternative hypotheses has been supported by detailed intracellular studies. Recent intracellular analyses of the membrane characteristics of FTG neurons have demonstrated that FTG cells are depolarized before and throughout REM sleep (Ito \& McCarley 1984; McCarley \& Ito 1983). McCarley (1978) has emphasized this point and coined the term "selectivity of modulation" to convey the idea that depolarization of the entire FTG cell population, not just subpopulations subserving particular movements, is a mechanistically critical and distinctive REM sleep phenomenon.

Although the kainic acid lesions clearly show that the FTG cells of the pontine reticular formation are neither necessary nor sufficient for initiating REM sleep, the results are at variance with the consistent finding that the rostral FTG, when pharmacologically stimulated, appears to function as a critical zone for triggering the electrographic signs of REM sleep (Baghdoyan, RodrigoAngulo, McCarley \& Hobson 1984; Drucker-Colin \& Bernal-Pedraza 1983). Furthermore, the lesion data do not rule out the possibility that the FTG might function as a REM sleep trigger zone under physiological conditions. There are numerous examples where lesions produce complex interpretative problems rather than supporting or refuting the hypothesis of a causal, regulatory role for a putative pattern generator. For example, selective lesions of the medullary dorsal and ventral respiratory nuclei do not eliminate breathing (Speck \& Feldman 1982), and lesions of the hypothalamic suprachiasmatic nuclei do not disrupt all circadian rhythms (Albers, Lydic, Gander \& Moore-Ede 1984; Fuller, Lydic, Sulzman, Albers, Tepper \& Moore-Ede 1981; Prosser, Kittrell \& Satinoff 1984; Szafarczyk, Ixart, Malaval, Nouguier-Soule \& Assenmacher 1979). In addition, surgical removal of well-defined epileptic foci does not always eliminate seizures in humans (Elazar \& Hobson 1985).

There are two important reasons for keeping these caveats in mind. One challenge to the interpretation that REM sleep is in part caused by FTG discharge was based on the finding that FTG neurons fire during waking movements and are thus not selectively active during REM sleep (Siegel \& McGinty 1977; Vertes 1977). A key observation, and one that tended to become lost in the discharge selectivity debate, is that the change in firing of FTG neurons precedes by several minutes the onset of REM sleep and all of its phasic phenomena. This long phase lead in FTG discharge prior to REM onset is difficult to explain as a mere correlate of phasic motor activity, since there is normally little or no motor activity during the early transition into REM sleep. No similar changes in FTG discharge have been reported during the transition from sleep to waking. On the contrary, after reaching an early peak the firing level of pontine reticular neurons falls progressively during the last half of each REM sleep episode and then decreases greatly as REM sleep gives way to wakefulness. Such a long tonic latency suggests that it would be useful to design experiments to explore the possibility of a change in FTG neuronal excitability level preceding REM onset (Ito \& McCarley 1984).

The second reason for continuing to consider the hypothesis that the rostral FTG is one of the regions from which REM sleep can be generated is that the anterodorsal pontine tegmentum is the only brain stem reticular site from which a behavioral state resembling REM sleep has been induced pharmacologically (Baghdoyan, Rodrigo-Angulo, McCarley \& Hobson 1982; Baghdoyan, Rodrigo-Angulo, McCarley \& Hobson 1986). These important data will be discussed in detail in Part IV of this paper, which explores the nature of the REM sleep activation process.

$b$. The cholinergic generator hypothesis. The postulated cholinergic identity of neurons has been confirmed in numerous brain stem areas from which REM-on cells have been recorded (Table 3D). In no case to date, however, has an electrophysiologically identified REMon cell also been confirmed to be cholinergic. Although it is not yet possible to demonstrate the presence of acetylcholine (ACh) in neurons, immunohistochemical identification of the synthetic enzyme choline acetyltransferase (ChAT) has been shown to be a reliable marker for cholinergic cells (Cuello \& Sofroniew 1984). The proposed cholinergic identity of pontine FTG cells has been supported (Kimura, McGeer, Peng \& McGeer 1981). This study showed that large cells in the rostral FTG stained heavily for ChAT, and Kimura et al. (1981) suggested that these cells are both cholinoceptive and cholinergic. However, the cholinergic nature of the pontine FTG cells remains to be confirmed using a monoclonal antibody for ChAT.

The density of ChAT-positive cells in the FTG and FTM has been found to be greater in the medullary than in the pontine extent of these reticular fields (FTG, FTL, FTM; Kimura et al. 1981). Sakai (1985c) has also demontrated that reticular cells in the ventromedial medulla (FTM) are ChAT immunopositive. These medullary cells (magnocellular or FTM of Berman) have recently been 
implicated in the generation of EEG desynchrony during REM sleep (Steriade, Sakai \& Jouvet 1984). Neurons in the FTM, interacting with pontine peri-LC alpha cells, have also been shown to play a role in generating the somatic muscular atonia of REM sleep (Sakai 1985c; Sakai, Sastre, Kanamori \& Jouvet 1981). Both the medullary FTM cells and the pontine peri-LC alpha neurons have been shown to be ChAT-immunopositive (Jones 1985; Sakai 1985c).

Of all the physiological signs of REM sleep, PGO waves have most convincingly been shown to be generated by cholinergic neurons. As discussed below, the cellular niechanisms responsible for generating PGO waves have been localized to the region surrounding the brachium conjunctivum near the junction of the pons and midbrain. Nuclei in this region consistently have been shown to contain cells that stain heavily for ChAT (Jones 1985; Mesulam, Mufson, Levey \& Wainer 1984; Sakai 1985c; Sofroniew, Priestley, Consolazione, Eckenstein \& Cuello 1985).

Finally, cholinergic neurons are known to play a role in generating the rapid eye movements of REM sleep. The ocular motor nuclei of the brain stem contain ChATpositive neurons (Cuello \& Sofroniew 1984; Kimura et al. 1981; Mesulam et al. 1984).

It thus seems justified to conclude that detailed physiological, pharmacological, and anatomical studies have substantiated many parts of this hypothetical picture of how REM sleep may be generated (Figures 1 and 3; Tables $1 \mathrm{~A}$ and $1 \mathrm{~B}$; Table 3 ). These results are consistent with the inference from the early transection studies (Jouvet 1962) that the pons plays a critical role during REM sleep in coordinating the activity of neurons in such widely separated sites as the cortex and the spinal cord.

\section{Neurons involved in generating the tonic and phasic} events of REM sleep. Superimposed upon the tonic (greater than $1 \mathrm{sec}$ ) background of a desynchronized electroencephalogram and postural atonia are the REM sleep phasic (less than $1 \mathrm{sec}$ ) events: the rapid eye movements themselves, the muscle twitches, and PGO waves recorded in the pontine reticular formation, the lateral geniculate bodies, and in the occipital cortices.

\section{a. The tonic events of REM sleep}

i. EEG desynchrony The original "paradox" of REM sleep was recorded by Jouvet and Michel (1959), who noted in a laboratory notebook: "The cat appears to be asleep but the EEG shows it to be awake!" Subsequent lesion, stimulation, and single-cell recording studies have indicated that this EEG desynchronization, which is characteristic of REM sleep but phenomenologically identical to that of wakefulness, is due to the following processes:

(1) Rostrally projecting neurons of the midbrain reticular formation are activated (Huttenlocher 196l; Kasamatsu 1970; Steriade 1981).

(2) These reticular neurons convey excitatory signals to the medial thalamus (Steriade, Ropert, Kitsikis \& Oakson 1980; Steriade \& Deschenes 1984) where

(3) the resulting depolarization of thalamic cells is relayed to cortical targets (Steriade 1981), including

(4) a wide variety of pyramidal and nonpyramidal cortical interneurons (Steriade 1978) and,
Hobson, Lydic \& Baghdoyan: Sleep cycle generation

(5) putatively excitatory and inhibitory neurons of the cortex (Steriade 1984).

Thus, at the level of extracellular recording, there is no visible difference in the mechanisms of cortical activation observed during REM sleep and waking. These findings vindicate and elegantly extend to the cellular level the concept of the ascending reticular activating system (Moruzzi \& Magoun 1949). The reciprocal-interaction model postulates that during REM sleep the reticulothalamocortical system is activated via the disinhibition associated with arrest of firing in the diffusely projecting (Azmitia \& Segal 1978) aminergic neurons, whereas during waking the cellular generators of EEG desynchronization must overcome aminergically mediated inhibitory restraint.

It has been shown that when parts of the midbrain reticular formation are microinjected with cholinergic agonists, the cortical EEG is desynchronized and the occurrence of the waking state is enhanced at the expense of time spent in REM sleep (Baghdoyan, RodrigoAngulo, McCarley \& Hobson 1984). It would thus be of particular interest to know how the aminergic neurons respond to cholinergic stimulation of the midbrain. The reciprocal-interaction model predicts no change or an increased aminergic cell discharge in association with midbrain cholinergic stimulation. Finding a significant reduction or a cessation of aminergic cell firing in such an experiment would weigh heavily against the reciprocalinteraction model.

ii. Muscular atonia During REM sleep in intact animals there is a powerful postsynaptic inhibition of the brain stem and final-common-path motoneurons that restrains all but the oculomotor movements, respiration, and phasic twitches of the skeletal musculature (Pompeiano 1967). Intracellular recordings from spinal motoneurons (Chandler, Chase \& Nakamura 1980; Glenn \& Dement 1981a; 1981b; Morales \& Chase 1978) have revealed tonic hyperpolarizations of up to $10 \mathrm{mV}$, as strong as the simultaneous depolarization of brain stem premotor effector neurons (Ito \& McCarley 1984). A likely suurce of such inhibition is the medullary reticular formation (Chase \& Morales 1984; Fung, Boxer, Morales \& Chase 1982), elements of which show the strongest tonic state-specific discharge yet observed (maximal discharge ratios for $\mathrm{REM} /$ waking of about 700/1; Netick, Orem \& Dement 1977). It has been proposed that these bulbar inhibitory neurons may be activated by the generalized disinhibition attributed to putatively aminergic REM-off cells and/or by specific excitatory projections from the anterodorsal pons (Sakai 1980). Compounding the obvious difficulty of performing long-term intracellular recordings in behaving animals, a major obstacle to obtaining critical evidence about the hypothesis of disinhibition is the difficulty of ascertaining distant effects as originating from the anatomically interpenetrated REM-on or REM-off populations (see Figures 1 and 2). b. The phasic events of REM sleep

$i$. PGO waves The PGO waves of REM sleep are associated with sequential phasic activation of cells in the medulla, pons, midbrain, thalamus, and cortex (Nelson, McCarley \& Hobson 1983; Orem 1980; Steriade \& Hobson 1976). The original descriptions and experimental analyses of PGO waves (Bizzi \& Brooks 1963; Brooks \& 
Bizzi 1963) suggested a strict temporal and spatial order of activation that has been substantiated by recent studies. For example, when the eyes move to the right during REM sleep, bursts of PGO-related cellular discharge occur in the right (and not the left) midbrain (Nelson et al. 1983), and the PGO waves of the right geniculate body and the right posterolateral cortex are larger in amplitude than those recorded from the left side of the brain (Monaco, Baghdoyan, Nelson \& Hobson 1984). Latency studies have revealed that the midbrain cellular discharge always precedes the ipsilateral geniculate wave onset by $11.0( \pm 2.45)$ milliseconds (Nelson et al. 1983).

The spatial and temporal specificity of PGO waves during REM sleep suggests that these patterns of electrical activity may represent an internal information system (Bowker 1985; Morrison 1979; Nelson et al. 1983) whose excitability is markedly enhanced during REM sleep. These findings are relevant to the efferent-copy hypothesis of von Holst and Mittelstaedt (1950) and of Sperry (1950). Both postulate an internal signal system by which the consequences of motor acts could be anticipated by sensorimotor systems whose inputs would necessarily be perturbed by the motor acts. [See also Berkinblit et al: "Adaptability of Innate Motor Patterns and Motor Control Mechanisms" 9(4) 1986.] 1985.)

ii. Eye movements The eye movements of REM sleep appear to be generated by the premotor neurons of the reticular formation (Henn, Buttner-Ennever \& Hepp 1982; Henn 1980; Henn, Hepp \& Buttner-Ennever 1982). These include pontine giant cells which have been shown to project remotely to the spinal cord and locally to oculomotor and vestibular neurons (Peterson 1980; Pompeiano 1980). These cells display the same range of firing patterns in REM sleep as in waking (i.e., short lead burst, long lead burst, and tonic discharge). However, this similar activation pattern does not guarantee identical mechanisms of activation or identical functional significance of these cells during REM sleep and wakefulness.

With respect to mechanisms activating the rapid eye movements, intracellular recording of pontine giant cells has revealed a powerful, tonic influence, with $10-\mathrm{mV}$ depolarizations in resting membrane potential reflecting excitation and/or disinhibition of these cells during REM sleep (Ito \& McCarley 1984). While the depolarization mechanism remains to be elucidated, disinhibition of the pontine giant cells by aminergic neurons is one possibility. This notion fits well with the state-specific cessation of midline aminergic neuronal activity during REM sleep (McGinty \& Harper 1976). This cessation of firing by aminergic neurons may provide a mechanism for REM-on cell activation that is analogous to the fundamental mechanism by which saccadic eye movements are generated in waking: in part through the cessation of midline omnipause neuron discharge (Keller 1974; Nelson et al. 1983).

iii. Muscle twitches The somatic musculature during REM sleep exhibits phasic twitches synchronized with bursts of eye movements. In the cat and in the human infant (Emde \& Metcalf 1970; Aaronson, Rashed, Biber \& Hobson 1982) the facial and digital limb and trunk muscles show this phasic activation to be in part patterned (in smiling, grimacing, and frowning) and in part sporadic (in clonic flexion and extension movements of whole body parts punctuating the REM sleep epoch). Recent investigations of the mechanisms that mediate these muscle twitches (Chase \& Morales 1983; Glenn \& Dement 1985) have focused on clarifying the relationship between descending excitatory input (Pompeiano 1967) and the hyperpolarization of alpha motoneurons, discussed above as a tonic event occurring throughout REM sleep. The intracellular studies suggest that during REM sleep alpha motoneurons are coactivated by both excitatory and inhibitor drives. Myoclonic muscle twitches during REM sleep are currently believed to result from brief episodes of synaptic excitation rather than the withdrawal of tonic inhibition.

The concept that central motor pattern generators are being activated during REM sleep (Pompeiano 1967) is supported by two further findings. One is the phasic firing of pyramidal tract, rubral, and brain stem motoneurons correlated with movement (Steriade \& Hobson 1976). The other finding is the dramatic expression of stereotyped and patterned motor acts by cats during a REM-sleep-like state that appears after bilateral lesions of the anterodorsal pontine tegmentum (Henley \& Morrison 1974; Jouvet \& Delorme 1965). During this REMsleep-like state, these lesioned cats walk and demonstrate sequences of stereotypic behavior similar to the defensive posturing and predatory stalking normally observed during wakefulness (Hendricks, Morrison \& Mann 1982; Morrison 1983).

In summary, the problems inherent in efforts to understand the cellular and molecular substrates underlying the tonic and phasic events of REM sleep (Vertes 1984) are not unique to neurophysiological investigations of behavioral-state control. For example, studies of respiratory rhythm generation show such complex interpenetration and overlapping of inspiratory and expiratory respiratory neurons that the discretely-defined-respiratorycenters concept has long been abandoned (Cohen 1979). In addition, recent reviews of the circadian-control system (Moore 1982; Groos 1984) report no data derived from single-unit recordings of the suprachiasmatic nucleus using intact, behaving animals. Thus, there is presently no empirical base to support cellular-level models of the otherwise extensively studied circadian system. For sleep neurophysiology, despite the foregoing limitations as to detail, the emerging picture is impressively coherent in suggesting that the sleep cycle is a function of dramatic and highly organized shifts in discharge activity of a widespread and complex neuronal network (Figures 1 and 5).

\section{B. The putative level-setting or modulatory population}

1. Identity and characteristics of the REM-off cells. In its original form the reciprocal-interaction model identified those cells of the LC with lowest activity levels in REM sleep as the highly localized, permissive component of the putative sleep cycle oscillator (Hobson et al. 1975; McCarley \& Hobson 1975b). These REM-off cells of the LC were postulated to exert a tonic restraint on the REMon cell population in the FTG by releasing the inhibitory neurotransmitter norepinephrine. Current revisions of 
the reciprocal-interaction hypothesis take account of new data indicating that ( 1 ) the REM-off cell populations are far more extensive than originally supposed (Figures 1 and 5; Table 1B); (2) at least one other aminergic neurotransmitter, serotonin, could jointly mediate the postulated modulatory action of the REM-off cells; and (3) the overlapping and interpenetration (Figure 2) of REM-on and REM-off cells seems to occur in many brain stem regions. The extent of the REM-on and REM-off interpenetration (Figures 1 and 2) may, therefore, serve to determine the discharge selectivity of a neuronal population during a given behavioral state.

REM-off cells were described in the dorsal raphe nucleus (DRN) (McGinty \& Harper 1976) at about the same time that they were discovered in the LC (Hobson, McCarley, Wyzinski \& Pivik 1973; Chu \& Bloom 1974a; 1974b). Since then REM-off cells have been found in numerous other brain regions. These include the putatively serotonergic nucleus linearis centralis and centralis inferior (Hobson, McCarley \& Nelson 1983), raphe pontis (Heyme, Steinfels \& Jacobs 1982), raphe pallidus (Sakai, Vanni-Mercier \& Jouvet 1983), raphe magnus (Cespuglio, Faradzi, Gomez \& Jouvet 1981), and the putatively noradrenergic peribrachial zone of the dorsolateral pontine tegmentum (Saito, Sakai \& Jouvet 1977; Sakai 1980). There is also a smattering of REM-off cells in the hypothalamus (Glotzbach \& Heller, personal communication, 1985), in the medullary reticular formation (Sakai et al. 1983) and in the central tegmental field of the pontine reticular formation (Hobson, McCarley \& Nelson 1983b), where the chemical identity of the REM-off cells is less certain than in those brain stem areas listed above.

The evidence concerning the aminergic identity of REM-off cells, though strong, is not yet absolutely conclusive. The assumption of aminergic identity is based on nuclear membership (Dahlstrom \& Fuxe 1965), distinctive spike train characteristics (McGinty \& Harper 1976; Aston-Jones \& Bloom 1981), proximity of REM-off cell recording sites to histochemically identified noradrenergic cells in the LC (Chu \& Bloom 1974a; 1974b), and arrest of firing after the administration of pharmacological agents that enhance serotonergic autoinhibition in the raphe (Gallager \& Aghajanian 1976). None of these points constitutes the certain, positive evidence of aminergic transmission that combined intracellular recording, dye staining, and/or immunocytochemistry could provide. Perhaps the most important piece of evidence in support of the noradrenergic nature of REMoff cells comes from the finding that the cells of the rat LC, an exclusively noradrenergic population, exhibit the REM-off firing pattern (Foote, Bloom \& Aston-Jones 1983).

The idea that the slowing and cessation of REM-off cell discharge before and during REM sleep may serve to release $\mathrm{REM}$-on neurons from aminergic restraint is supported by evidence suggesting that norepinephrine and serotonin are inhibitory neurotransmitters (Foote et al. 1983; Gallager \& Aghajanian 1976). However, the axonal projection patterns of many REM-off neurons do not indicate particularly dense innervation of the reticular regions in which the most highly selective REMon cells are found (Sakai 1980). In addition, as is well known, aminergic neurons project to regions remote

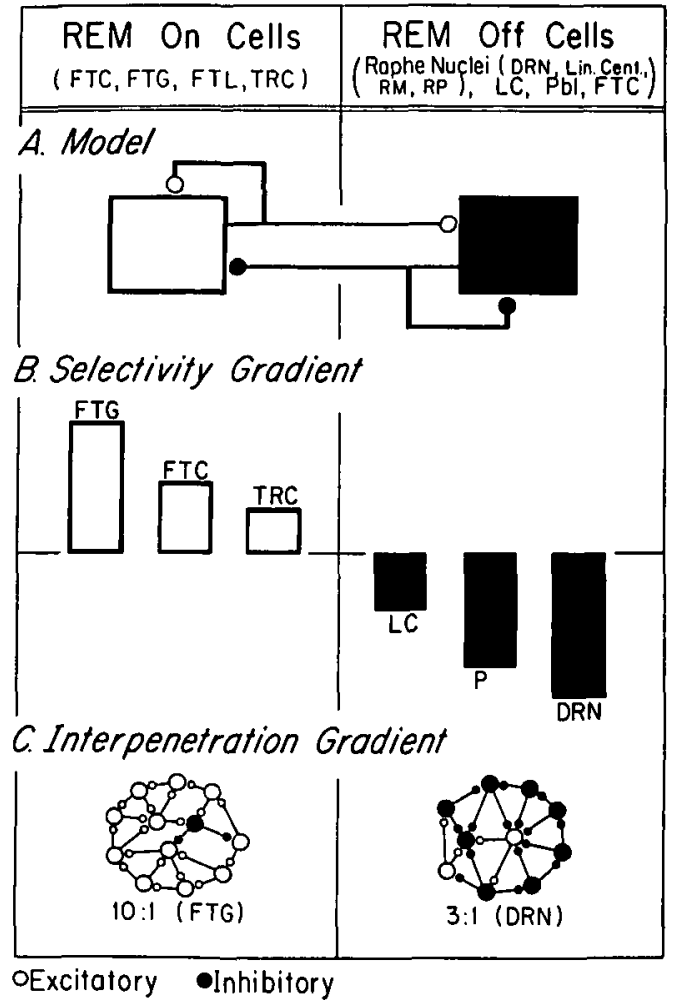

Figure 2. Relationship between interpenetration and selectivity. Schematic illustration of the hypothesized relationship between physiological selectivity (B) and anatomical interpenetration (C) of REM-on (left column) and REM-off (right column) cells whose reciprocal interconnections are modeled in A. REM-on cells are those neurons whose discharge rate in REM sleep is at least twice that of waking and NREM sleep. REM-off cells are those neurons whose discharge rate is at most one-half that of waking and NREM sleep. Inhibitory neurons are represented by filled circles; excitatory neurons are represented by open circles. The same caveats regarding connectivity and synaptic function advanced in Figure 1 apply to this figure.

The model (A) shows the REM-on cells as having feedforward and feedback (auto) excitation while the REM-off cells have feedforward and feedback (auto) inhibition. The neurotransmitters postulated to mediate these effects are shown in Figure 5C.

Selectivity (B) is defined as the ratio of the mean firing rate of a given population in REM sleep relative to the firing rate during waking without movement. The proportional strengths of the values (not given) are accurately represented. Note that the significantly positive selectivity values of the REM-on populations and the significantly negative values of the REM-off populations form a continuous gradient.

The interpenetration (C) of excitatory and inhibitory cells (or synapses) in a given nucleus may determine the selectivity gradient shown in B since the proportions of REM-on and REMoff cells in a nucleus correlates with the selectivity ratios. In the examples shown, REM-on cells exceed REM-off cells by 10 to 1 in the area with the greatest positive selectivity (e.g., the FTG) while REM-off cells conversely exceed REM-on cells by 3 to 1 in the nucleus with the greatest negative selectivity (e.g., the DRN).

Anatomical nomenclature following Berman (1968):

REM-on nuclei: FTG = gigantocellular tegmental field; FTC $=$ central tegmental field $;$ FTL $=$ lateral tegmental field; TRC $=$ tegmental reticular nucleus.

REM-off nuclei: DRN = dorsal raphe nucleus; Lin Cent $=$ linearis centralis; $\mathrm{RM}=$ raphe magnus; $\mathrm{RP}=$ raphe pontis; $\mathrm{LC}$ $=$ locus coeruleus; $\mathrm{P}=$ peribrachial region. 
from the brain stem, especially the cortex and spinal cord (Azmitia \& Segal 1978; Tohyama, Sakai, Salvert, Touret \& Jouvet 1979; Tohyama, Sakai, Touret, Salvert \& Jouvet 1979). The fact that REM-off cell projections are not exclusive to the REM-on cell zones indicates that some aspects of the postulated reciprocal interaction are anatomically distributed, not only at the level of the brain stem but also throughout the brain. The possibility of anatomically distributed (Figure 1) and histochemically interpenetrated (see Figure 2C) REM-off and REM-on cell populations gives the reciprocal-interaction concept an even more distributed field of action (Figure 2A) than the original discussion of the REM-on cell data had implied (Hobson et al. 1975; McCarley \& Hobson 1975b). This leads logically to the consideration that sleep cycle control may involve a system with both localized and remote cellular elements, both of which may play significant regulatory roles under physiological conditions.

One example will suffice to illustrate the functional implications of a reciprocal interaction between the anatomically distributed systems thought to be involved in sleep cycle control. As noted above, the eye movements of REM sleep are generated by premotor neurons in the brain stem reticular formation and by final-common-path oculomotor neurons whose own excitability may also be increased by disinhibition (Henn, Baloh \& Hepp 1984). However, the increase in firing that causes this system to generate bursts of saccades during REM sleep may derive from (1) remote excitatory inputs to the cortex and superior colliculi, (2) excitation from recurrent collaterals, and (3) local vestibular feedback. Thus, a variety of neurons related both locally and remotely to the saccade-generating cells may be rendered more excitable by the disinhibition postulated to result from the REM-off firing pattern of aminergic neurons.

The hypothesis that recruitment of the distributed REM-on population is centripetal (e.g., via corticopontine fibers) as well as centrifugal (e.g., via pontogeniculate and pontospinal pathways) may account for the important finding that the eye movements of REM sleep are less intense after lesions of the colliculi or visual and motor cortices (Jeannerod, Monet \& Jouvet 1965) and that electrical stimulation of the lateral geniculate body can trigger PGO waves and REM sleep if stimulation is delivered during the transition period from NREM to REM sleep (Nelson, McCarley \& Hobson 1978). In addition, it has been shown that REM latency can be decreased and REM duration can be increased by auditory stimulation, which increases PGO waves (DruckerColin, Bernal-Pedraza, Fernandez-Cancino \& Morrison 1983).

The relative ratio of interpenetration between $\mathrm{REM}$-on and REM-off cells in the brain stem of the cat (Figure 2C) may also be associated with the degree to which both of these populations reveal discharge patterns selective for a given behavioral state (Figure $2 \mathrm{~B}$ ). For example, the most impressive REM-off selectivity is seen in the DRN, where the proportion of cells that cease firing in REM sleep (rather than merely slow their discharge) is highest and REM-on cells have not been reported (Figure 2B, right). Conversely, REM-on selectivity in headrestrained animals is greatest in the FTG, where REM-off cells have not been recorded (Figure $2 \mathrm{~B}$, left). Finally, in regions where there is an admixture of $\mathrm{REM}$-on and REM-off cells (such as the FTC), both classes of neuron show a relatively weak tendency to discharge selectively in a given behavioral state.

A parsimonious explanation of this apparent gradient of discharge selectivities would be a parallel gradient in density of net excitatory versus inhibitor synaptic contacts (Figure 2C). According to this concept, the functional homogeneity of cell type (i.e., REM-on or REMoff) in a nucleus should enhance the tendency of that nucleus to discharge with either a REM-on or REM-off selectivity pattern (Figure $2 B$ ). Thus, REM-on and REMoff discharge selectivity in a brain stem region appears to be, in part, a function of local homogeneity. In other words, this local homogeneity may take the form of uniformly signed (i.e., excitatory or inhibitory) local projections that would tend to bias the balance of competing excitatory and inhibitory inputs of remote origin.

\section{Deactivation of REM-off cells: Inferences derived from} correlative data. According to the reciprocal-interaction concept, arrest of firing by aminergic REM-off cells disinhibits REM-on cells and this process is critical in determining the REM sleep state. Our continued tests of the foregoing hypotheses have recently focused upon the pontine DRN. The DRN represents an ideal site for testing these hypotheses since it has been shown (1) to contain the highest concentration of serotonin in the brain (Dahlstrom \& Fuxe 1965), (2) to be the source of most of the forebrain serotonin (Azmitia \& Segal 1978), and (3) to be a component of an extensive collection of monoaminergic nuclei that extend from the medulla to the mesencephalon. Furthermore, DRN neurons show a regular discharge during wakefulness, a slowing in NREM sleep, and a near cessation of firing during REM sleep.

So robust is the negative correlation between DRN discharge and REM sleep that the cessation of raphe discharge has consistently been observed in a variety of experimental preparations. For example, extracellular recordings of DRN discharge reveal a cessation of firing during the REM-sleep-like state induced by cooling the locus coeruleus (Cespuglio et al. 1981). A slowing of DRN firing was observed during the REM-sleep-like state without atonia produced by lesions of the pontine tegmentum (Trulson, Jacobs \& Morrison 1981). Pharmacological studies have revealed a REM-off discharge in the DRN during the REM-sleep-like state produced by pontine injections of carbachol (Steinfels, Heym, Strecker \& Jacobs 1983). Finally, the DRN has been shown to exhibit a REM-off firing pattern in recordings from oneday-old kittens, before the DRN has completed its biochemical and anatomical development (Adrien \& Lanfumey 1984).

Similarly, if one reviews the cellular studies of DRN discharge during physiologically occurring REM sleep, a slowing and cessation of DRN discharge has always been reported (Heym et al. 1982; Hobson, McCarley \& Nelson 1983; Lydic, McCarley \& Hobson 1984; McGinty \& Harper 1976; Trulson \& Jacobs 1979). There have been no exceptions to the observation of a REM-off discharge in studies using intact, undrugged adult animals. This decremental discharge pattern, therefore, appears to 
represent one of the most consistent findings in sleep neurophysiology: In the intact, undrugged animal, DRN discharge is always positively correlated with wakefulness and negatively correlated with REM sleep. Considered together, these data strongly support the working hypothesis that the DRN plays a causal role in modulating the temporal organization of REM sleep or in disinhibiting the generators of specific REM sleep signs, such as muscular atonia or PGO waves.

Nevertheless, the hypothesis that REM-off discharge patterns may be causally related to REM sleep was recently opposed by the notion that the REM-off firing pattern is only correlated with REM sleep. The latter suggestion arose from the finding that the arrest of firing by REM-off cells was significantly reduced by experimentally placed lesions in the pontine tegmentum (Trulson et al. 1981). One group of these lesions reduced the cessation of firing by DRN cells and produced a REM-sleeplike state without atonia. During this state, DRN cells were still observed to discharge less than in wakefulness but approximately four times as much as during physiological REM sleep. These findings led to a suggestion that the dorsal raphe's REM-off discharge pattern might be a consequence of somatic muscular atonia (Trulson et al. 1981).

Subsequent long-term recordings from the DRN of intact, undrugged cats demonstrated that the REM-off discharge pattern of the DRN was not monotonically related to the muscular atonia of REM sleep. (Lydic, McCarley \& Hobson 1983a) These data also showed that the activity profiles for motor atonia and DRN discharge are not uniformly parallel, as would be expected if feedback from muscle activity were a dominant or significant control mechanism regulating DRN discharge. There are no data concerning a possible direct relationship between DRN discharge and hyperpolarization of alpha motoneurons, but results of long-term recordings from REMoff cells raise the question whether the physiological mechanisms generating atonia are the same or different from the cellular mechanisms that regulate the timing of atonia (I,ydic, McCarley \& Hohson 1985).

For some time the causation-versus-correlation question with respect to $\mathrm{REM}$-off cells has focused on data describing the rate of neuronal discharge averaged within wakefulness or stages of sleep. Only recently have data become available concerning the time course of REM-off discharge displayed by DRN cells across a large sample of temporally unified sleep cycles (Lydic, McCarley \& Hobson 1986a; 1986b). The distinction between rate and time-course analyses is important since time-course profiles of cellular discharge provide information concerning the phase relationships between the putative regulating and regulated variables. Time-course analyses have revealed the phase relationships among DRN discharge, PGO-wave activity, and the atonia of REM sleep to be consistent with the hypothesis that the DRN plays a role in temporally coordinating the physiological events of REM sleep (Lydic et al. 1983a; 1984; 1985; 1986a; 1986b).

Forced locomotor activity (treadmill exercise) has recently been used to manipulate the temporal characteristics (period, amplitude, and phase) of the sleep cycle. These experiments examined the null hypothesis that there is no consistent phase relationship between the sleep cycle and the DRN discharge cycle. Three lines of evidence consistent with the hypothesis that the DRN is involved in sleep cycle modulation have emerged from these studies: (1) Although forced activity was capable of significantly altering the duration and percentage of wakefulness, NREM, and REM sleep, the time course of the ultradian sleep cycle could not be dissociated from the time course of DRN discharge (Lydic et al. 1984). (2) Spectral analyses of DRN discharge and simultaneously recorded $\mathrm{PGO}$ waves revealed that the period lengths of both PGO waves and DRN discharge were simultaneously shortened following exposure to forced locomotor activity (Lydic et al. 1985). This finding was consistent with evidence suggesting that DRN discharge levels modulate the timing of PGO waves. For example, PGO waves were increased by lesions that isolated the midline DRN from lateral brain stem areas (Simon, Gershon \& Brooks 1973). PGO waves were markedly increased by pharmacological manipulations that disrupted serotonin synthesis, storage, or neurotransmission (Jouvet 1972). (3) Time-course and regression analyses have both revealed that although DRN discharge is of a REM-off nature, there is a phase-dependent increase in DRN firing that occurs during the latter stages of REM sleep in anticipation of wakefulness (Lydic et al. 1984; 1985). Since a cause must precede its effect, these data strongly support the hypothesis that the DRN is causally involved in modulating the temporal organization of the ultradian sleep cycle and/or specific REM sleep events such as PGO waves or muscle tone.

By what cellular mechanisms do aminergic neurons, of which DRN cells are but one example, cease discharging before and during REM sleep? Pacemaker potentials in the DRN have recently been identified (Aghajanian \& Lakoski 1984), and their possible role in the REM-off discharge pattern remains to be explored. Several lines of evidence suggest a pontine reticular influence on DRN neurons. Long-latency inhibition of aminergic neurons was reported in anesthetized rats after electrical stimulation of the FTG component of the medial pontine reticular formation (Wang, Gallager \& Aghajanian 1976). It is known, however, that general anesthetics hyperpolarize some vertebrate neurons (Nicoll \& Madison 1982), and in the unanesthetized cat FTG stimulation actually enhanced DRN discharge (Heym, Steinfels \& Jacobs 1984). Furthermore, the ability of FTG stimulation to enhance DRN discharge was found to be statespecific in the unanesthetized cat (Lydic et al. 1983b). DRN discharge was maximally enhanced when the FTG was stimulated during wakefulness. Stimulation of FTG during NREM sleep was only moderately effective and DRN discharge was unchanged when the FTG stimulation was administered during REM sleep.

These observations all bear on the hypothesized excitatory action of the REM-on cells on the REM-off cells. Although they generally support this assumption, they also show that the efficacy of such excitability is not constant across the sleep cycle. As it stands, the reciprocal-interaction model has no way of accounting for the observed refractoriness of DRN cells to excitatory stimuli during REM sleep. Since disfacilitation is an unlikely explanation, we must consider as a strong possibility an active inhibitory mechanism arising in the 
raphe itself, or inhibition mediated by an as yet unidentified interneuron.

Pharmacological studies have shown that the activity levels of DRN discharge can be differentially modified by centrally and peripherally acting cholinomimetics (Steinfels et al. 1983). GABA-ergic mechanisms are also known to influence raphe activity (Gallager \& Aghajanian 1976; Nishikawa \& Scatton 1983) and may play some role in the REM-off mechanism. Finally, as noted below in Section D of Part V, numerous peptides have been jointly localized within known aminergic nuclei and may play some role in the REM-off discharge pattern. It is, therefore, critically important for future studies to determine the degree to which these various cellular and molecular mechanisms might mediate the REM-off discharge pattern and state-dependent alteration of DRN excitability.

\section{Comparison of the reciprocal-interaction model with other models of sleep cycle control}

In the preceding section we discussed in detail specific postulates of the 1975 version of the reciprocal-interaction model, which were subsequently shown to be incorrect and in need of revision or abandonment. Our reevaluation of the model has also included a review of the many functional (Table 1A) and structural (Table 1B) postulates of the model that have been supported. As a third step in this reevaluation process, we now turn to a comparison of the reciprocal-interaction model with other explicit formulations of sleep cycle control. Through this comparison we show that, despite some differences in detail, there is striking agreement regarding both the empirical base and the conceptual essence among all available models concerning the neural regulation of sleep (Figure 3 and Table 2). A comparison of Figures 1 and 2 with Figure 3 and Table 2 makes this point clear.

Component models (Figure 3A, Table 2) have been concerned with the physiological generation of specific components of REM sleep, such as rapid eye movements, PGO waves, or somatic muscular atonia. For example, Pompeiano and Valentinuzzi's (1976) vestibular transform model (Figure 3Al) schematized the processes believed to underlie the generation of rapid eye movements. The Pompeiano-and-Valentinuzzi model is similar to the reciprocal-interaction model in that it is based on cellular discharge data that have been mathematized in terms of excitatory-inhibitory interactions. A tonic excitatory drive is postulated to be cholinergic, to arise in the pontine reticular formation, and to be opposed by tonic inhibitory restraint originating from aminergic nuclei of the brain stem. According to this model, the tonic excitatory drive is converted into phasic pulses through the activation of well-known reticulovestibulooculomotor circuitry subserving the vestibulooculomotor reflex and nystagmus.

On the basis of lesion and pharmacological data, PGO waves were postulated by Simon et al. (1973) and RuchMonachon, Jalfre, and Haefely (1976) to be generated by paramedian pontine reticular neurons. During wakeful- ness, these pontine neurons were further postulated to be held in inhibitory restraint by serotonergic raphe and/or noradrenergic locus coeruleus "gating" mechanisms (Figure 3A2). We note that in the models of Simon and RuchMonachon the term "gate" implies the inhibitory restraint that the reciprocal-interaction model specifically ascribes to the firing of REM-off cells.

The models of REM sleep atonia (Figure 3A3) proposed by Chase (personal communication 1985) and Morrison (1983) share with the reciprocal-interaction model an acceptance of Pompeiano's (1967) original suggestion that the postsynaptic inhibition of anterior horn cells is mediated by descending fibers of brain stem origin. All the models in Table 2 that consider motor inhibition during REM sleep agree that the most likely source of such inhibition is the medullary reticular formation. Chase does not specify the workings of the "pontine gate" by which normally excitatory responses to stimulation are converted to inhibitory ones during REM sleep, but he sees the pontine reticular formation and locus coeruleus as somehow involved. Morrison's model schematizes brain stem systems that are hypothesized to inhibit movement during REM sleep. This model also postulates the pontine activation of a medullary inhibitory center and further proposes pontine inhibition of the facilitative lateral locomotor strip. The medullary inhibitory center inhibits alpha motoneurons, with the net result being atonia.

Integrative models (Figure 3B, Table 2) include those that, like the reciprocal-interaction model, attempt to account for the constellation of the physiological and behavioral events that occur during REM sleep. This integrative approach was pioneered in the late 1960s by Jouvet. Figure 3 illustrates Jouvet's (1978) conceptual scheme; its rich complexity need not obscure the important postulation of "pontine driver" neurons whose excitability is modulated by noradrenergic locus coeruleus and serotonergic raphe inputs. Jouvet illustrates the postulated relationship between hypothetical pacemaker neurons ( 1 in Jouvet's schema) and pontine driver interneurons (shown as 2 and 3 in Jouvet's illustration) which are "submitted to gating effects from the locus coeruleus or raphe nuclei or to modulatory influences from the frontal cortex or the vestibular nuclei" (p. 249).

The integrative model of Sakai (1984) postulates a paradoxical sleep (PS) "center" comprising PS-on and PSoff cells analogous to the REM-on and REM-off cells of the reciprocal-interaction model. These cells are hypothesized to be mutually excitatory and inhibitory, as in the reciprocal-interaction model, but with their synaptic signs reversed. The net effect is a model very similar to the reciprocal-interaction model (Figures 1 and 5), and the differences between these two models can be tested experimentally (see also Table 3 and Part IV).

Hybrid models such as Borbély's two-process model use specific concepts derived from a variety of data sources (Figure 3C, Table 2). These concepts have been eclectically combined to produce an explanatory model of sleep cycle control. Borbély's (1982) model is unique in its effort to incorporate data from sleep deprivation studies, data concerning the circadian rhythm of core body temperature, data concerning the temporal organization of sleep, and neurophysiological data derived from the 


\section{A. COMPONENT MODELS}

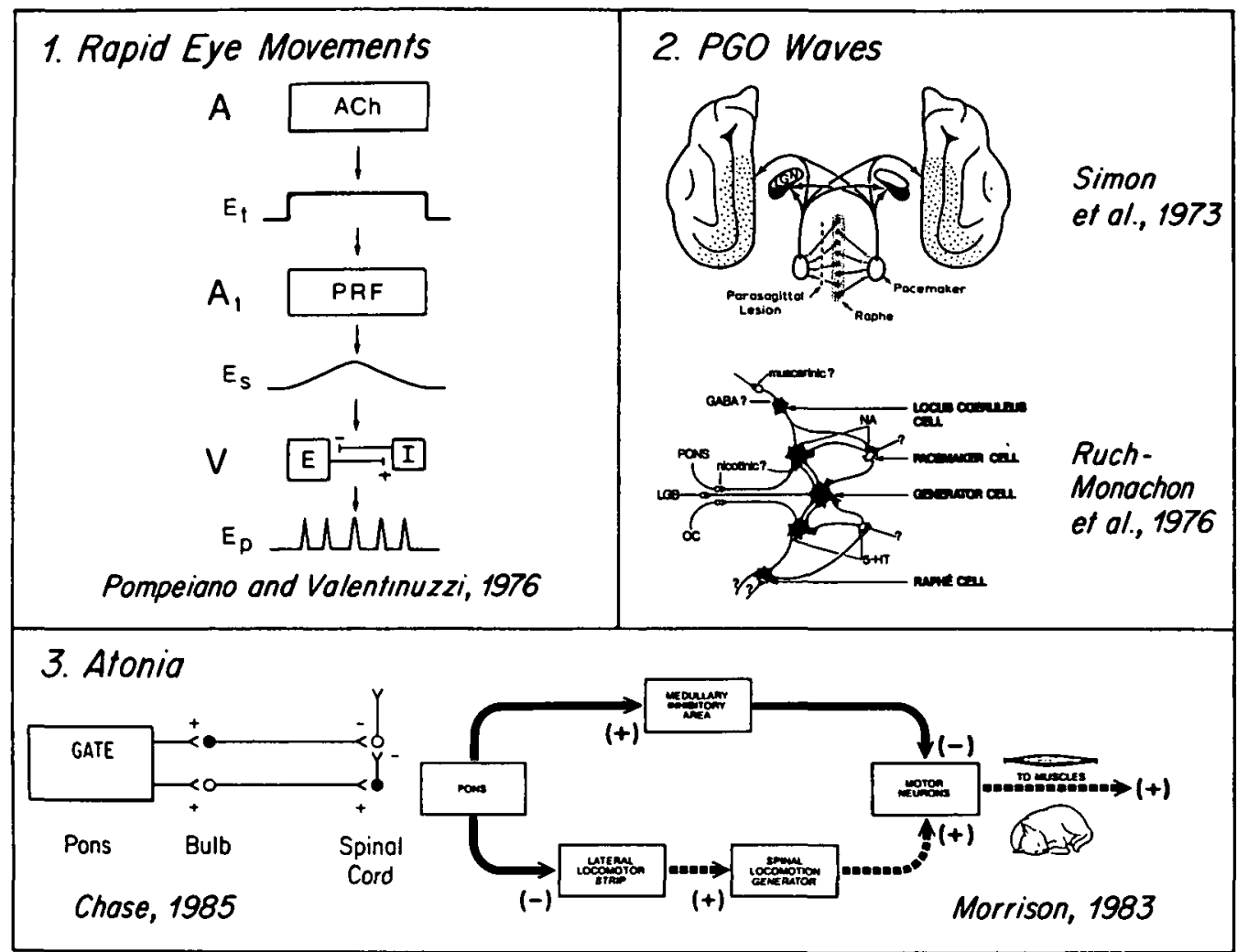

B. INTEGRATIVE MODELS

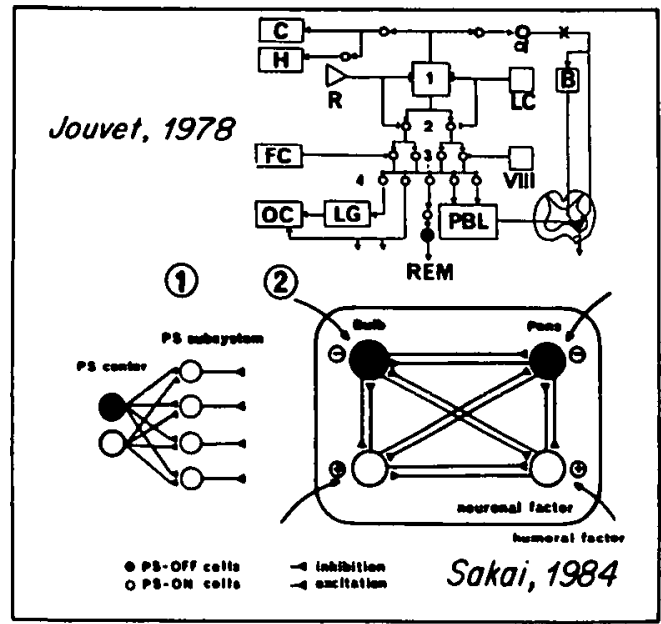

Figure 3. Schematic models of sleep cycle generation. A comparative view of eight models of the neural processes postulated to be involved in sleep cycle generation. These models share numerous conceptual similarities with the reciprocalinteraction model (see text and Table 3).

Schematic representations of the generation of physiological components of REM sleep, such as rapid eye movements, PGO waves, and somatic muscular atonia, are illustrated as Component Models (Figure 3A). Pompeiano and Valentinuzzi's (1976) model postulated a self-excitatory cholinergic system (A) that has a tonic $(t)$ excitatory output (Et). The dorsolateral pontine tegmentum (PRF) was postulated to transform the tonic output into an oscillating signal (Es). In the vestibular nuclei (V), reciprocally interacting excitatory $(\mathrm{E})$ and inhibitory $(\mathrm{I})$ neurons were postulated to produce a rhythmically phasic output $(\mathrm{Ep})$ generating the rapid eye movements. Schematic reproduced from Pompeiano \& Valentinuzzi (1976) by permission.

Prior to single-unit recordings from the dorsal raphe nucleus
C. HYBRID MODELS

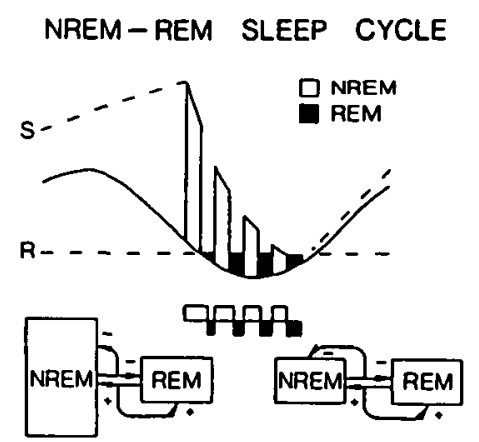

Borbely, 1982

(raphe), Simon et al. (1973) postulated that PGO-wavegenerating mechanisms (pacemaker) were inhibited by the discharge of serotonin-containing (5-HT) neurons in the raphe. Reproduced from Simon et al. (1973) by permission.

Detailed pharmacological studies of PGO wave generation also led Ruch-Monachon et al. (1976) to hypothesize that noradrenergic (NA) and serotonergic (5-HT) cellular discharge inhibited PGO wave generation by pontine cholinergic and cholinoceptive neurons. This model also proposed a modulatory role for gamma-aminobutyric acid (CABA) exerted on the locus coeruleus neurons. Reproduced from Ruch-Monachon et al. (1976) by permission.

Models of brain stem mechanisms postulated to generate the somatic muscular atonia of REM sleep are illustrated in Figure 3A3. Chase and Morales (1984) proposed a model of statedependent somatomotor control that is schematically illustrated by the model described to us by Chase (personal communication, 1985). At the level of the spinal cord, this model illustrates 
the inhibition of alpha motoneurons that mediate the muscular atonia of REM sleep. The supraspinal origin of this atonia is currently thought to involve an interaction between pontine and bulbar areas that serves to transform (or gate) excitatory $(+)$ into inhibitory (-) output in a REM-sleep-dependent manner.

Morrison's (1983) model illustrates inhibitory $(-)$ and excitatory $(+)$ connections in the brain stem hypothesized to inhibit movement during REM sleep. According to Morrison's schematic: "In normal REM sleep the pons strongly activates the inhibitory center in the medulla (top). The midline inhibitory zone in the pons inhibits the lateral locomotor strip. The result is complete paralysis" (p. 102). Modified after Morrison (1983) by permission.

Integrative models of sleep cycle control (Figure 3B) include those which aim to integrate conceptually the various physiological and behavioral components comprising the sleep cycle. Jouvet's (1978) model postulated that the components of REM sleep are mediated by effector neurons (4) which are, in turn, activated by pontine pacemaker (1) and driver interneurons ( 2 and 3). The pontine driver interneurons were postulated to receive "gating effects" from the locus coeruleus (LC) or raphe (R) nuclei "or modulatory influences from the frontal cortex (FC) or the vestibular nuclei (VIII)." One group of effector neurons related to the visual system was postulated to ascend to the lateral geniculate (LG) and occipital cortex (OC). The rapid eye movements (REM) were postulated to be mediated by another group of effector neurons, and neurons involved in PGO wave generation were postulated to include neurons in the nucleus parabrachialis lateralis $(\mathrm{PBL})$. This model proposed that the motor atonia of REM sleep is mediated by a motor inhibitory system in the locus coeruleus (alpha) that descends directly or indirectly in a pathway $(\mathrm{X})$ to relay neurons in the bulbar (B) reticular formation and then to the alpha motoneurons in the spinal cord. Reproduced from Jouvet (1978) by permission.

Sakai's (1984) model schematizes cellular systems hypothesized as the "central mechanisms underlying the generation of paradoxical sleep (PS) subsystems (1) and for the central mecha- nisms involved in the generation of PS (2)." Reproduced from Sakai (1984) by permission.

Hybrid models (Figure 3C), such as Borbély's (1982) twoprocess model of sleep regulation, have selectively combined data from a variety of sources. Borbely's formulation produced a computer model that generated time-course plots of two operationally defined processes postulated to be involved in sleep cycle regulation: a sleep-dependent process (S) and a circadiandependent process, equivalent in this illustration to REM sleep propensity (R). Reproduced from Borbély (1982) by permission.

reciprocal-interaction model. The mechanisms proposed by Borbély (1982) to account for the cyclic alternation of NREM and REM sleep are identical to those of the reciprocal-interaction model.

The postulate of dynamic interaction between cholinergic enhancement and aminergic suppression of REM sleep generation was anticipated by the pharmacological model of Karczmar, Longo, and De Carolis (not illustrated but described by Table 2). The interactional postulates of the reciprocal-interaction model have also been applied successfully by computer-simulation studies investigating the relationship between depression and changes in the temporal organization of sleep (Beersma 1984; Beersma, Daan \& Van den Hoofdakker 1984; see Table 2).

Considered together, these diverse conceptual models of sleep cycle control share many features with the reciprocal-interaction model (Figure 3 and Table 2). The commonalities strongly emphasize the unifying power of the reciprocal-interaction model. This unifying power favors the retention of an appropriately revised reciprocal-interaction model since the central goal of all

Table 2. Comparison of the reciprocal-interaction model with other models of sleep cycle control

\begin{tabular}{|c|c|c|c|c|c|c|}
\hline \multirow[b]{2}{*}{ Model of: } & \multicolumn{6}{|c|}{ Characteristics of the reciprocal-interaction model: } \\
\hline & $\begin{array}{l}\text { Pontine } \\
\text { generation } \\
\text { of REM } \\
\text { sleep signs }\end{array}$ & $\begin{array}{l}\text { Specification } \\
\text { of putative } \\
\text { generator } \\
\text { neurons }\end{array}$ & $\begin{array}{l}\text { Specification } \\
\text { of putative } \\
\text { modulatory } \\
\text { neurons }\end{array}$ & $\begin{array}{l}\text { Dynamic } \\
\text { interaction } \\
\text { of REM-on and } \\
\text { REM-off cells }\end{array}$ & $\begin{array}{l}\text { Dynamic inter- } \\
\text { action of } \\
\text { cholinergic and } \\
\text { aminergic cells }\end{array}$ & $\begin{array}{l}\text { Mathematical } \\
\text { model of perio- } \\
\text { dicity based on } \\
\text { cellular discharge }\end{array}$ \\
\hline $\begin{array}{l}\text { Pompeiano \& Valen- } \\
\text { tinuzzi } 1976\end{array}$ & + & + & + & + & + & + \\
\hline $\begin{array}{l}\text { Simon et al. } 1973 \\
\text { Ruch-Monachon et al. }\end{array}$ & + & + & + & - & + & - \\
\hline $\begin{array}{l}1976 \\
\text { Chase, personal com- }\end{array}$ & + & + & + & - & + & - \\
\hline munication, 1985 & + & + & - & - & - & - \\
\hline Morrison 1983 & + & + & - & - & - & - \\
\hline Jouvet 1978 & + & + & + & + & + & - \\
\hline Sakai 1984 & + & + & + & + & + & - \\
\hline $\begin{array}{l}\text { Karczmar et al. } 1970 \\
\text { Beersma 1984; }\end{array}$ & + & - & - & - & + & - \\
\hline Beersma et al. 1984 & + & + & + & + & + & + \\
\hline Borbély 1982 & + & + & + & + & + & + \\
\hline
\end{tabular}

Key: $+=$ Postulate present; $-=$ postulate absent.

Note: This table summarizes the presence $(+)$ or absence $(-)$ of six postulates of the reciprocal-interaction model in 10 other models of sleep cycle control. Details regarding the degree of similarity or difference between these models and the reciprocalinteraction model are provided in the text and in Figure 3. 
model building is to explain the greatest number of empirical facts by the smallest number of assumptions.

\section{Postulated mechanisms of reciprocal interaction: Causal hypothesis testing via experimental intervention}

In previous sections of this paper we have shown how the capacity to record from single cells in behaving animals has yielded data supporting numerous models of sleep cycle control, many of which share the assumption of dynamic interaction between cholinergic and aminergic neuronal populations. We have further specified some interpretive difficulties related to the inability of correlational data to establish a causal role for the postulated control mechanisms. It seems to us that what is needed to advance the field is the development of experimental techniques that simultaneously (1) allow the investigator to manipulate the excitability of discrete neuronal populations; (2) recognize and accommodate the known anatomical distribution, interpenetration, and chemical specificity of the neuronal populations under investigation; and (3) overcome the crudeness, nonspecificity, and side effects known to occur with lesioning and electrical stimulation of the CNS.

The recent development of a reliable means for inducing a REM-sleep-like state by central administration of cholinergic agonists gives a novel form of experimental control to the study of basic sleep mechanisms. This control makes causal hypothesis testing easier and allows direct and systematic study of such critical questions as:

1. Which cell populations, when pharmacologically influenced, produce or suppress REM sleep?

2. What neurotransmitters and what receptors mediate these effects?

3. What are the cellular and molecular mechanisms of progressive excitation throughout the anatomically distributed REM-on cell population?

\section{A. Experimental induction of a REM-sleep-like state}

New concepts about possible processes activating REMon cells have emerged from experiments using the technique of chemical microinjection of cholinergic drugs to evoke a state polygraphically and behaviorally similar to physiological REM sleep (Baghdoyan, Rodrigo-Angulo, McCarley \& Hobson 1986; Baghdoyan et al. 1982; Baghdoyan, Rodrigo-Angulo, McCarley \& Hobson 1984). Chemical microinjection offers several advantages over electrical stimulation, most notably the capacity to influence cell bodies while not affecting fibers of passage. Chemical stimulation also allows for activation or blockade of specific populations of neurotransmitter receptors. For example, the behavioral and electrographic properties of the REM sleep state can be faithfully mimicked by microinjecting the muscarinic cholinergic receptor agonists bethanechol (Hobson, Golberg, Vivaldi \& Riew 1983) and dioxolane (Vivaldi \& Hobson 1981) into the pontine brain stem. Furthermore, the REM-sleep-signenhancing effects of neostigmine, an inhibitor of acetylcholinesterase, can be blocked by central administration of the muscarinic receptor antagonist atropine (Baghdoyan, Monaco, Rodrigo-Angulo, Assens, McCarley \& Hobson 1984), and microinjection of the muscarinic receptor antagonist scopolamine blocks carbachol-induced REM sleep sign enhancement (Shiromani \& McGinty 1983).

More relevant to the more general themes of this paper is the fact that the behavioral and electrographic syndrome produced by microinjection of cholinergic agonists depends on the site of drug administration in the brain stem (Figure 4). The pontine reticular zone from which the polygraphic signs of REM sleep can be elicited is large but clearly bounded, being bordered by midbrain and medullary REM sleep suppression zones (Baghdoyan, Rodrigo-Angulo et al. 1984). In the pontine region from which a REM-sleep-like state can be evoked, the electrographic syndrome produced by microinjection of cholinergic agonists may be similar to or different from physiological REM sleep, according to the anatomical site of drug administration (Baghdoyan et al. 1982).

The drug-induced REM-sleep-like state of longest duration, shortest postinjection latency to onset, and most consistent polygraphic similarity to physiological REM sleep is elicited by carbachol microinjections made near the interface of three reticular regions in the paramedian anterodorsal pons (Figure $4 \mathrm{~A}$ ). These reticular regions have been shown to include the gigantocellular tegmental field, central tegmental field, and tegmental reticular nucleus (Baghdoyan et al. 1982). These data emphasize that the most effective site is not in the center of the FTG, as the original form of the reciprocal-interaction model had predicted.

There is a second, distinctly localized PGO generation zone in the peribrachial region of the lateral anterodorsal pons (Figure 4B; Vivaldi et al. 1980). When microinjected with cholinergic agonists, the neurons of this region fire in bursts that lead the PGO waves, just as these neurons discharge during physiologically occurring REM sleep (Nelson et al. 1983; Vivaldi et al. 1980).

A third zone is in the posteroventral pontine reticular formation, from which microinjection of carbachul produces stereotyped alternating eye movements and PGO waves, together with other electrographic REM sleep signs and REM-sleep-like behavior (Baghdoyan, Rodrigo-Angulo et al. 1984; Figure 4C). At anatomical regions where these pharmacologically defined REM sleep enhancement and suppression zones border one another, dissociations of polygraphic sleep signs may occur: atonia with arousal (at the pontomesencephalic border; Mitler \& Dement 1974); rapid eye movements with arousal (at the pontomedullary border; Baghdoyan et al. 1982; Figure 4D). These findings are consistent with the neurophysiological data supporting the concept of an anatomically distributed, functionally differentiated, but chemically specific REM sleep generator population.

The chemical microinjection technique has also shown that the anatomically distributed REM-on population can be functionally dissected. For example, by microinjecting carbachol into different brain stem regions it is possible selectively to activate muscular atonia (Steinfels et al. 1983), state-independent PGO waves (Vivaldi et al. 1980; Figure 4B), or state-independent rapid eye movements (Baghdoyan et al. 1982; Figure 4D). The finding that the putative generators of the components of REM sleep can 


\section{ELECTROGRAPHIC SYNDROMES PRODUCED BY CARBACHOL ARE DEPENDENT UPON MICROINJECTION SITE}
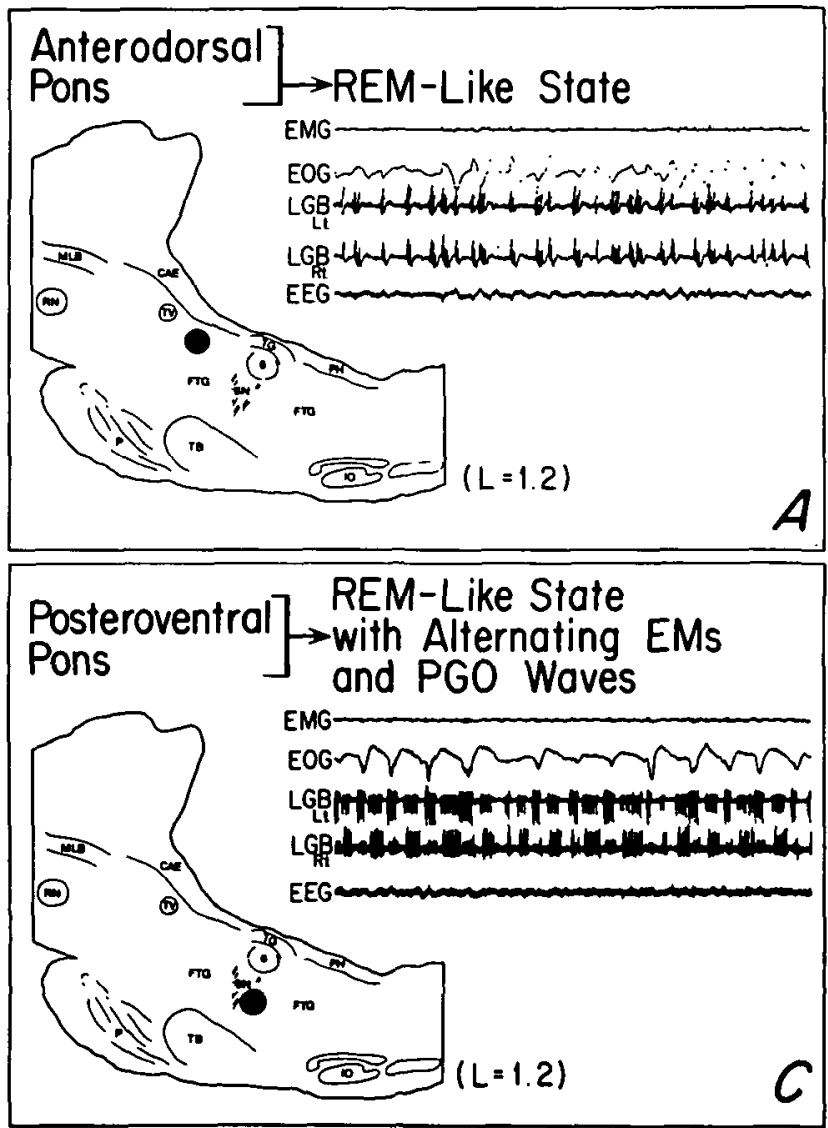

Figure 4. Differential effects of intra-brain stem cholinergic stimulation. Sagittal schematic drawings of the cat brain stem showing the electrographic syndromes evoked by microinjection of carbachol into four different brain stem regions. Solid circles in the brain stem schematics indicate loci of injection. $\mathrm{L}$ is the lateral coordinate of the brain stem section in $\mathrm{mm}$, according to the atlas of Berman (1968). A. Microinjection of carbachol into the rostral pontine reticular formation repeatedly elicited a state that resembled REM sleep, both polygraphically and behaviorally. $B$. When injections were made more rostrally and laterally, into the peribrachial region of the pons, continu-
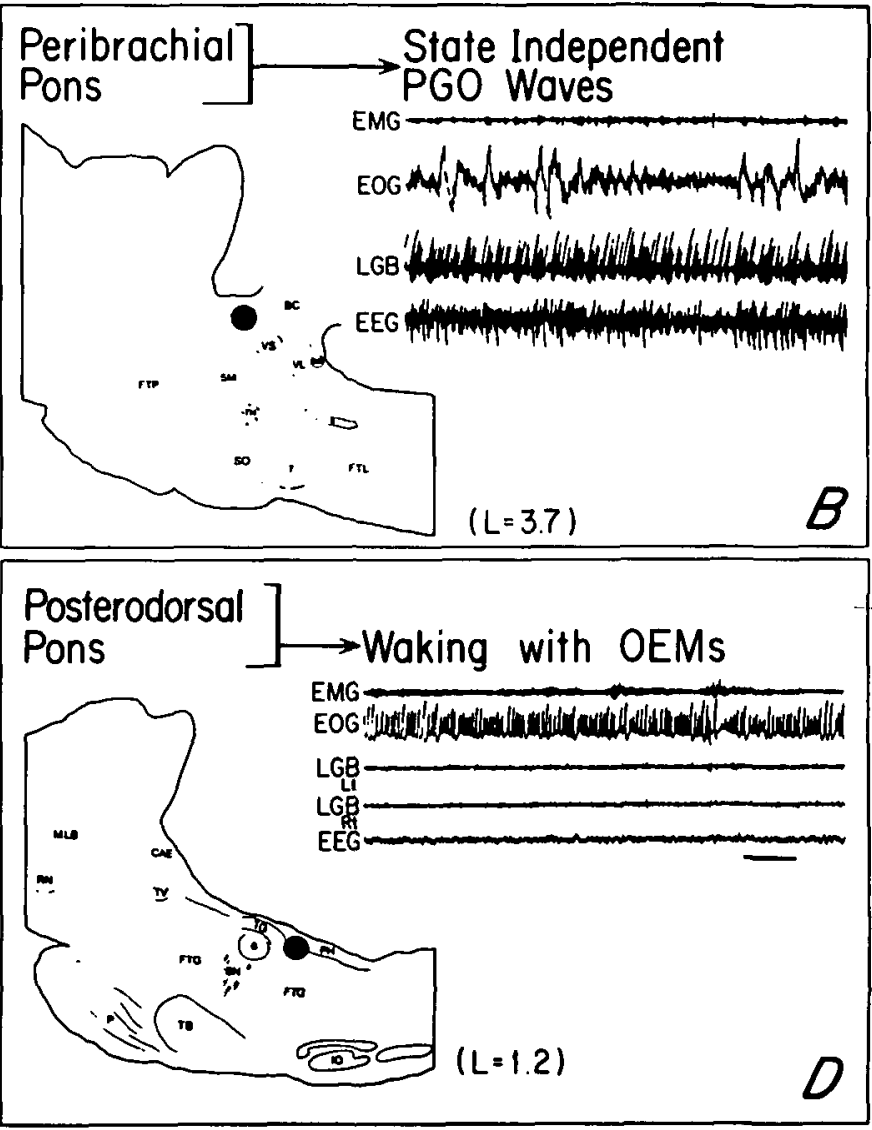

ous PGO waves were produced that occurred independently of behavioral or other electrographic signs of REM sleep. $C$. Carbachol injection into the posteroventral pontine reticular formation evoked a REM-sleep-like state characterized by a unique pattern of $P G O$ wave and rapid eye movement clusters that alternated direction from left side to right side. $D$. Carbachol microinjection into the posterodorsal pons, along the pontomedullary junction, suppressed behavioral and electrographic signs of REM sleep and produced a state of wakefulness initially characterized by unidirectional or oscillatory, repetitive rapid eye movements (OEMs). be activated independently of the state of REM sleep suggests that under physiological conditions there is a temporally organized recruitment of these putative generator populations. This temporal organization would allow for sequential activation of the individual generator populations so as to produce the unified REM sleep state. A possible mechanism of temporal organization could be disinhibition of the REM-on cell population by the putatively aminergic REM-off cells.

This concept of an optimal recruitment sequence among putative generator populations is also supported by the data showing that microinjection of cholinergic agonists into parts of the postulated REM sleep generator network can produce suppression, rather than enhancement, of REM sleep. For example, it has been shown that after microinjections of carbachol in the posterodorsal pons, unilateral rapid eye movements are produced and REM sleep is abolished for at least four hours (Baghdoyan et al. 1982; Figure 4D). It thus appears that if the part of the putative REM sleep generator network that controls rapid eye movements is activated before other components (e.g., the PGO wave generator or the motor atonia generator), or if it is activated with an abnormal intensity or pattern, then the normal production of REM sleep is blocked. This finding may reflect a drug-induced disruption of the hypothesized temporal organization between the various neuronal populations that interact to produce the coordinated physiological signs of REM sleep. In other words, temporal dissociations between such highly interactive systems could oppose the orderly recruitment of neuronal populations. These data imply that, under physiological conditions, the recruitment of the dis- 
tributed neuronal populations comprising the putative REM sleep generator network may normally proceed in a temporally ordered sequence.

The finding that the REM sleep generator populations can be pharmacologically activated to produce electrographic syndromes not observed under physiological conditions (e.g., Figures 4B, 4C, 4D) is presumably due to the intrinsic features of the pharmacologically influenced neurons. For example, microinjection of carbachol into the posteroventral pontine reticular formation evokes tonic manifestations of REM sleep that are normal (such as motor atonia), but instead of clusters of eye movement saccades (i.e., the rapid eye movements), stereotyped side-to-side alternating eye movements and associated PGO waves are evoked (Figure 4C). The unidirectional, stereotyped rapid eye movements generated by carbachol microinjection into the posterodorsal pons (Figure 4D) presumably result from activation of the preoculomotor neurons of the nucleus praepositus hypoglossi. Thus, variation in both the behavioral and the physiological manifestations of the cholinoceptively triggered REM-sleep-like state may be related to specific anatomical portions of the putative REM sleep generator network that are directly activated by the administered drugs.

These findings further clarify the emerging picture of a large but finite REM sleep trigger zone in which functionally differentiated neuronal components must be recruited in a temporally and spatially specific order for REM sleep to develop normally. Among other things, this new schema of the delicate dynamics of REM sleep generation helps explain why REM sleep has been so easy to disrupt and so difficult to enhance. Most of the concepts suggested by the results of the microinjection studies remain to be tested empirically. The experimental control afforded by chemical microinjection will allow investigators to answer many of the specific questions raised in this paper.

\section{B. Heuristic value and limitations of the pharmacologically induced REM-sleep-like state}

Microinjection of cholinergic agonists into the pontine reticular formation produces a state similar to physiological REM sleep in that it is characterized by four classic electrographic criteria: muscular atonia, PGO waves, rapid eye movements, and EEG desynchrony. While these electrographic signs are present the animals are behaviorally asleep. In spite of these similarities between physiological REM sleep and the pharmacologically evoked REM-sleep-like state, many variables remain to be tested for identity or differences. For example, it would be interesting to know whether, during the cholinoceptively evoked REM-sleep-like state, the values of physiological variables such as core body temperature, brain temperature, heart rate, and blood pressure and behavioral variables such as arousal threshold are similar to those occurring during physiological REM sleep. Preliminary data suggest that arterial blood pressure decreases during the cholinoceptively induced REM-sleeplike state (Shiromani, Siegel \& McGinty 1984), as it does during physiological REM sleep. Similarly, it is essential to know what is occurring at the cellular discharge level during the cholinoceptively evoked REM-sleep-like state. Do all of the REM-on cells fire? Do all of the REM-off cells cease firing? Experiments to answer these questions are now being performed using a combination of the chemical microinjection and microwire recording methods.

Even if some of these variables are shown to differ during the two states (and many of them can actually be expected to differ), it is our view that the pharmacologically evoked REM-sleep-like state would remain valuable as an experimental tool. Under physiological conditions the reducing firing rate of the aminergic neurons of the LC and DRN prior to and during REM sleep is hypothesized to release the putative REM sleep generator neurons from inhibitory restraint. Under pharmacological conditions, however, it may not be mandatory that all aminergic cells slow or cease firing in order to allow for the elaboration of the cholinoceptively evoked REM-sleep-like state. What may be necessary, under either pharmacological or physiological conditions, is that the level of excitation in the REM-on cell population be able to overcome the tonic inhibition hypothesized to be imposed by the REM-off cells. It is theoretically quite possible that one part of the anatomically distributed REM-sleep-generating system, if driv: en intensely enough by a local injection of a cholinergic agonist, could overcome any putative inhibitory influence from the aminergic cells and produce a REM-sleeplike state while the aminergic cells remained active.

It is also likely that the firing rate of the REM-on cells will differ substantially during pharmacologically evoked and physiological REM sleep. Those REM-on cells which are directly excited by cholinergic agonists may fire at higher rates than normal. It is even conceivable that excitation by the agonist will cause such strong depolarization that firing will decrease. Conversely, REMon cells not directly excited chemically may not be recruited due to the continued inhibition postulated to arise from the aminergic cells and may fire at levels that are lower than under physiological conditions. Thus, it is hypothesized to be the ratio of discharge levels between REM-on and REM-off cell populations (rather than absolute levels) that determines behavioral state.

\section{Causal hypothesis testing}

During the past 10 years the study of sleep cycle generation has continued to be pursued primarily in a descriptive experimental mode. To some extent this descriptive approach has been mandated by the size of the mammalian CNS, the complexity of implicated physiological control mechanisms, and the dynamic temporal aspects of sleep cycle organization. Although the cartography of state-dependent cellular excitability changes remains incompletely documented, numerous reviews (Steriade \& Hobson 1976; Vertes 1984; Hobson \& Steriade 1986) make it clear that this descriptive decade has been productive enough to warrant now the formulation of experimental predictions and to move more vigorously toward causal hypothesis testing.

Experience with the cholinergic microinjection approach encourages extending this method to testing the 
aminergic tenets of the model as well as to documenting the cellular correlates of the chemically induced states. We hope that other innovative approaches to causal hypothesis testing will be offered in the peer commentaries stimulated by this paper.

\section{Current status and future development of the reciprocal-interaction model}

In this section we summarize and illustrate the changes in the reciprocal-interaction model necessitated by the specific challenges to the model's original tenets reviewed in Part II, the comparisons with other models reviewed in Part III, and the new data presented in Part IV.

Figure 5 has thus been designed with two goals in mind: (1) to show the similarities and differences between the 1975 version of the model (Figure $5 \mathrm{~A}$ ) and our present view (Figure $5 \mathrm{C}$ ) occasioned by the increase in the data base (Figure $5 \mathrm{~B}$ ), and (2) as a result of the increase in the data base (Figure 5B), to provide as detailed a picture as is currently possible of how each component of the REM sleep state may be generated at the cellular level (Figure 5D; compare with Figure 1).

As a prelude to detailing the specific means of testing the newly formulated model in Table 3, we discuss the previously reviewed findings in structural and dynamic terms. We then anticipate the ways in which current cellular methods and the reciprocal-interaction concept could be applied to understanding circadian influences on the sleep cycle and conclude with a discussion of how the new data derived from sleep-disorders medicine may be organized in terms of the reciprocal-interaction model.

\section{A. Structural considerations of the reciprocal-interaction model}

1. Cellular components. The initial emphasis on the pontine reticular giant cells and locus coeruleus cells as critical elements of the REM sleep generator system led to a rigid and restricted view of the model's cellular components that now appears incorrect. It is now clear that neither of these neuronal groups is either necessary or sufficient for behavioral-state control. One goal of the present reformulation is to modify the reciprocalinteraction model according to the evidence indicating that neuronal populations residing in many nuclear groups constitute the components of the model (see Figures 5B and 5D).

2. Synaptic connections. Corresponding to the rigidly restricted view of cellular components was the suggestion of synaptic interconnections between the two original component candidates, the FTG and the LC. Although convincing evidence supporting the functional significance of these interconnections has been reported (see Table 1), the pathways are neither as unique nor as restricted as implied by the original formulation of the reciprocal-interaction model (compare Figures $5 \mathrm{~A}$ and 5C). It can be seen that its essential connectionistic assumptions may be subserved by (1) a multiplicity of weak interconnections and (2) a multiplicity of neuronal subcomponents.

\section{B. Dynamic considerations of the reciprocal-interaction model}

The dynamic assumptions of the reciprocal-interaction model are given by the Lotka-Volterra equations (Davis 1962; May 1973; McCarley \& Massaquoi 1985), which generate curves displaying a close fit to the discharge profiles displayed by the REM-on and REM-off cell populations. The adequacy of these equations to account for the periodic occurrence of these cellular discharge profiles is both unique and compelling. This modeling approach is retained because of its predictive powers and because no other model, based upon empirically derived neurophysiological data, has attempted to mimic the periodicity of the cellular activity underlying the sleep cycle.

In its initial formulation, the reciprocal-interaction model did not specify the physical basis of the time constant, and the period length of the sleep cycle remains unaccounted for by the current revision. However, the schematic representations of Figures 1 and 5 should not be read as limiting our modeling of the time constant to merely synaptic effects. On the contrary, since it is known that the period length of the sleep cycle varies with the size of the sleeper's brain, it may be speculated that an intracellular, metabolic event (such as protein synthesis or transport) also contributes to the time constants.

The effects on the model of perturbations of the CNS, such as alterations in synaptic strength or phase angle shifts related to differing times of sleep onset, have recently been simulated by McCarley and Massaquoi (1985) with special reference to the data on sleep in depression and in relation to the circadian system. In these computer simulations, the model responds in accordance with the Vogel et al. (1980) description of the reduced REM sleep latency in depression. Additional clinical application of the reciprocal-interaction model are considered in the final part of this paper and in Table 4.

\section{Experimental predictions of the reciprocal-interaction model}

Table 3 summarizes the experimental predictions of the reciprocal-interaction model, both those that have been tested and those remaining to be tested. To understand the interpretations given in Table 3 it will be useful to compare them to Figure $5 \mathrm{D}$, which represents our most detailed and current schematic picture of the REMsleep-generating neuronal system.

Table 3A summarizes selected electrophysiological studies in which REM-on and REM-off cells were recorded across the sleep cycle. One assumption of the original reciprocal-interaction model, that the FTG fires selectively in REM sleep, was refuted by several investigations. However, the predictions of tonic latency, phasic latency, and tonic changes in REM-on cell membrane potential have all been confirmed.

Another prediction of the original reciprocalinteraction model was that the REM-off cell discharge 
Hobson, Lydic \& Baghdoyan: Sleep cycle generation

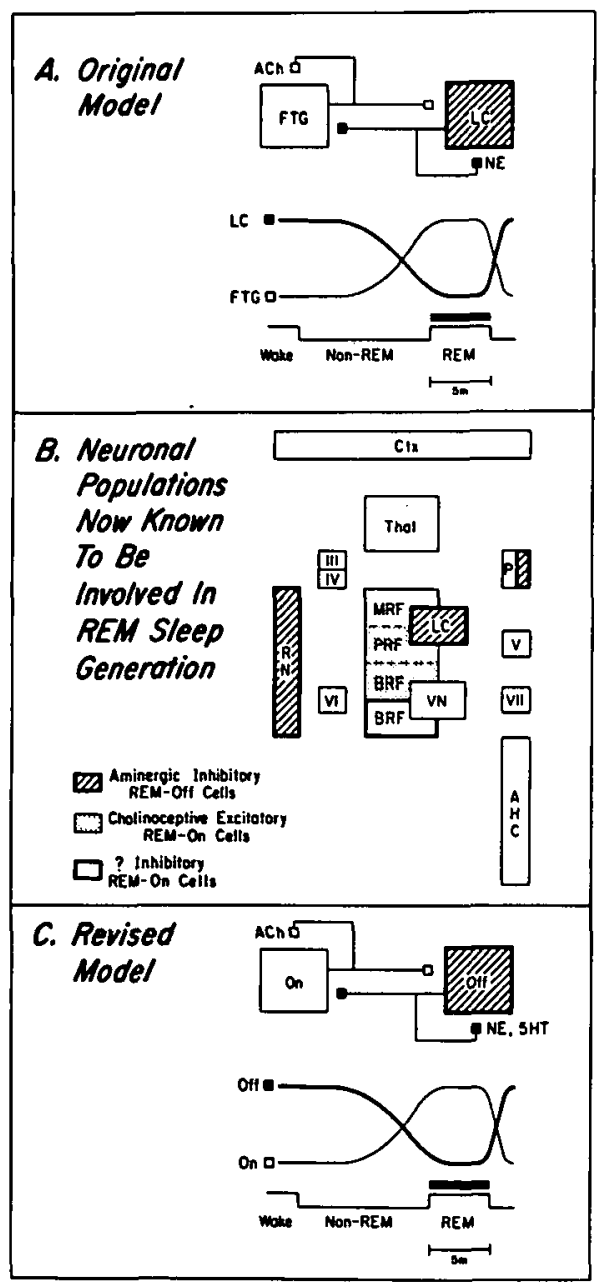

Figure 5. Schematic summary of the REM sleep generation process.

Figure 5A. Original reciprocal-interaction model. In 1975 this model was restricted to one class of REM-on cell, the putatively cholinoceptive/cholinergic neurons of the gigantocellular tegmental field (FTG), and one class of REM-off cell, the noradrenergic neurons of the locus coeruleus (LC). The FTG was assumed to exert feedforward and feedback excitation via cholinergic synapses (ACh). The LC was assumed to exert feedforward and recurrent inhibition via noradrenergic synapses (NE). The reciprocal activity curves labeled LC and FTG illustrate the discharge rate (spikes/sec) of these two neuronal populations over the sleep cycle (shown as Wake, Non-REM, and REM sleep). The two neuronal discharge curves were well fitted by the Lotka-Volterra equations. Time calibration, $5 \mathrm{~min}$.

Figure $5 B$. Schematic summary of neuronal populations presently known to be involved in REM sleep generation. The coordination of populations of REM-on cells (stippled boxes) may be achieved via changes in the level of inhibitory restraint imposed by the REM-off cells (cross-hatched boxes). Also shown are other neuronal groups known to be activated in REM sleep (open boxes). This diagram and its expanded presentation in Figure 5D integrate the data and concepts of Figure 1 (the distributed nature of both the modulatory and generator populations), Figure 2 (the interpenetrated nature of REM-on and REM-off cells), and Figure 4 (the pharmacological activation of either the entire REM sleep generator population or the subcomponents associated with generating the specific physiological manifestations of REM sleep).

The three known loci of REM-off cells (cross-hatched boxes) are the raphe nuclei (RN, serotonergic), the locus coeruleus (LC, noradrenergic), and the peribrachial region ( $P$, noradrenergic). Each REM-off cell group has recurrent collaterals

\section{Cell Groups Medioting REM Sleep Signs}

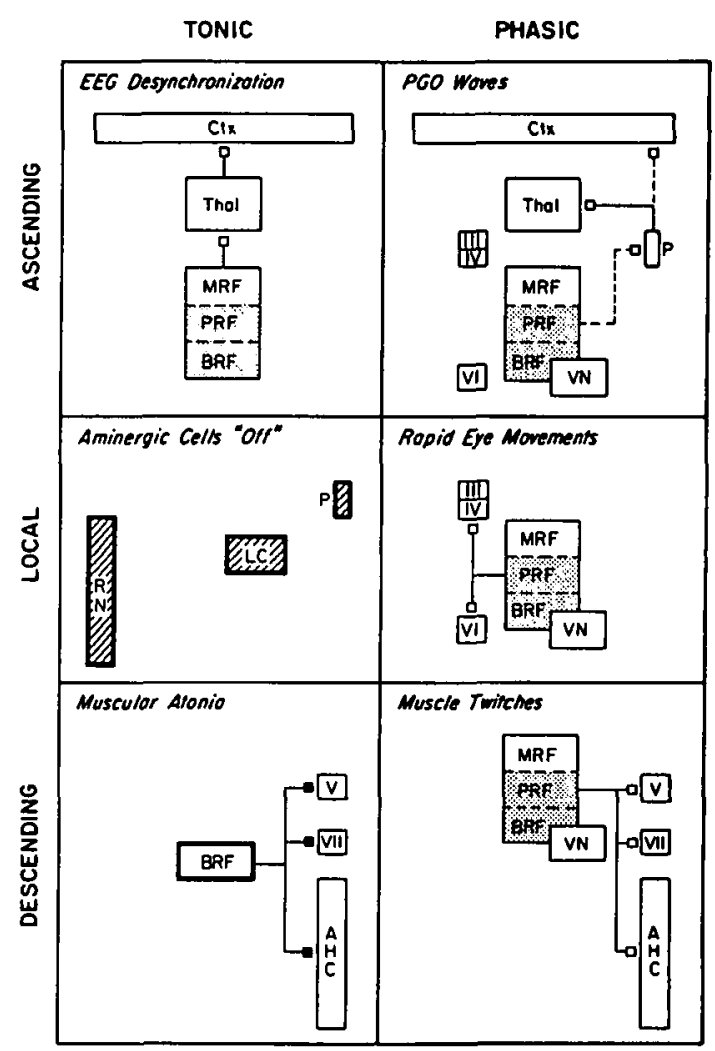

(see Figures 5A and C), and the REM-off cell groups are also interconnected (see Tables $1 \mathrm{~A}$ and $\mathrm{B}$ ).

The loci of REM-on cells are organized as follows: The group of reticular formation (RF) neurons is subdivided into its midbrain (MRF), pontine (PRF), and bulbar (BRF) components; note that the latter group is further subdivided into excitatory (light lines) and inhibitory (heavy lines) populations. The vestibular nuclei (VN) are superimposed to indicate their wellknown interconnections with the RF and the oculomotor nuclei (III, IV, and VI) via the medial longitudinal fasciculus (not shown). The other brain stem nuclei shown are the trigeminal (V) and the facial (VII). Rostral REM-on populations include the PGO burst cells of the peribrachial zone (P), the thalamus (Thal), and the cerebral cortex (Ctx). The caudal populations of segmental motoneurons are the anterior horn cells $(\mathrm{AHC})$ of the spinal cord.

Interpenetration of REM-on and REM-off cells is most prominent in rostral dorsal brain stem. Thus, the $L C$ is superimposed on the reticular formation at the junction of the MRF and PRF, and the peribrachial zone $(\mathrm{P})$ is presented as a mixture of REMoff and PGO burst cells.

Figure 5C. Revised reciprocal-interaction model. The basic form of the model is the same, but the interacting populations now include all REM-on cells (most of which are still assumed to be cholinoceptive and cholinergic) and all REM-off cells (some of which are now thought to be serotonergic).

Figure 5D. Expanded schematic of neuronal population interaction during the generation of REM sleep signs. The tonic events of REM sleep are illustrated in the three frames on the left. Neuronal systems mediating the phasic events of REM sleep are shown in the three frames on the right of Figure 5D. The frame on the top left of Figure 5D schematizes EEG desynchronization as the result of a tonic barrage of excitatory impulses arising 
in the rostral mesencephalic and pontine reticular formation and projecting to the thalamus and cortex. This schematic does not illustrate many synaptic interactions now known to occur both within the thalamus and between the thalamus and cortex. PGO waves are the result of phasic barrages of excitatory impulses arising in the reticular formation, oculomotor, and vestibular nuclei and propagated rostrally to the thalamus and cortex. Muscular atonia is the result of a tonic barrage of inhibitory impulses arising in the caudal reticular formation (BRF) and propagated to the brain stem motor nuclei (V and VIII) and the anterior horn cells (AHC) of the spinal cord. Muscle twitches are the result of a phasic barrage of excitatory impulses arising in the reticular formation, oculomotor, and vestibular nuclei and propagated caudally to the brain stem motor nuclei and the AHC of the spinal cord. Rapid eye movements are the result of phasic barrages of excitatory impulses arising in the reticular formation (MRF and PRF) and the vestibular nuclei (VN). The aminergic cells of the RN, LC, and P cease discharging during REM sleep (Off).

pattern of LC neurons was state-selective. As noted in Part II of the text, this LC REM-off discharge pattern has now been confirmed in rodents. Table $3 \mathrm{~A}$ shows that serotonergic cells of the DRN also meet tonic latency, phasic latency, and selectivity predictions of the reciprocal-interaction model.

Table 3B outlines predictions of the model that are testable by altering the activity of the REM-on and REMoff cell populations. Pharmacological manipulations that presumably increase REM-on cell activity (central administration of cholinergic agonists) have been shown to produce a REM-sleep-like state, while administration of pharmacological agents that presumably decrease the activity of REM-on cells (cholinergic antagonists) inhibits REM sleep. Eliminating FTG cells, which were originally proposed to be the REM sleep executive neurons, did not abolish REM sleep, and Table $3 B$ shows that this prediction of the original reciprocal-interaction model was not confirmed.

Dynamic manipulations of the REM-off cell population have, in general, supported the predictions of the reciprocal-interaction model. For example, Table 3B summarizes data showing that presumed inactivation of REM-off cells by cooling the LC and DRN enhanced REM sleep, and presumed activation of LC REM-off cells by $\mathrm{ACh}$ prevented the occurrence of REM sleep. Presumed pharmacological inactivation of LC neurons, however, did not increase REM sleep, contrary to the predictions of the model. The section of Table $3 \mathrm{~B}$ referring to REM-off cells indicates that many predictions of the reciprocal-interaction model remain to be tested by dynamic manipulations of REM-off cell activity.

Table $3 \mathrm{C}$ lists the synaptic relationships postulated to exist between the REM-on and REM-off cells of the pons. The electrophysiological data summarized in Table $3 \mathrm{C}$ support the existence of these synaptic relationships and are thus consistent with, but do not demonstrate, the hypothesis that reciprocal interaction occurs. Supporting evidence awaits simultaneous recording of synaptically interacting REM-on and REM-off cells in combination with an experimental demonstration that pharmacological manipulation of either cell group influences the other in a predicted manner. As indicated in Table 3C, several other postulated synaptic relationships remain to be tested.
Finally, Table 3D lists predictions of the model concerning the histochemical identity and neurotransmitter receptivity of the REM-on and REM-off cell populations. The predictions of neurotransmitter receptivity for the REM-on cells remain to be confirmed. As noted in this portion of Table 3 and in Part I of the text, the assumption of the reciprocal-interaction model that REM-off cells are aminergic was based, in part, upon the histochemical studies of Dahlstrom and Fuxe (1965). Table 3D also shows that, for the most part, iontophoretic studies of REM-off cells have supported the predictions about their responsiveness to various neurotransmitters.

Considered together, these four portions of Table 3 show that the reciprocal-interaction model is rich in its predictive powers and that many of these predictions are experimentally testable. Such conclusive tests as have been performed support the main tenets of the model. The major disconfirmation concerns the original postulate of the FTG as the exclusive site of the putative REM sleep generator. As we have acknowledged in Part IV, the FTG does not appear to be necessary, nor is its activation sufficient, to trigger REM sleep. This means that either some other, as yet undiscovered REM-on cell population is critical or (as is postulated in this paper) the REM-on cell network is anatomically distributed and interpenetrates with REM-off cells that activate the REM-on cells by disinhibition.

\section{Circadian control of the sleep state oscillator: Hypotheses regarding mechanisms}

In its original formulation, the reciprocal-interaction model did not attempt to deal with the problem of the circadian nature of the sleep cycle, despite the absence of REM sleep during the circadian activity phase and the presumed activation of a putative REM sleep oscillator during the circadian rest phase. Relevant to understanding this problem are recent developments in the basic neurobiology of circadian rhythm control by the suprachiasmatic nucleus (SCN) of the hypothalamus (e.g., Moore 1982).

The following observations may be germane to understanding the relationship between brain stem control of the sleep cycle and hypothalamic control of circadian rhythms. A strong serotonergic input from the pontine brain stem to the hypothalamic SCN has been demonstrated (Azmitia \& Segal 1978). Ultradian oscillations in discharge levels of the DRN, documented by spectral analyses (Lydic et al. 1984; 1985a), could provide temporal information about sleep cycle period length and relative phase to the $S C N$ component of the putative hypothalamic circadian oscillator through the pathways described by Azmitia and Segal (1978). Cosinor analyses revealed that serotonin levels in the DRN fluctuate with a significant circadian rhythm (Semba, Toru \& Mataga 1984). In addition, the secretion of many hypothalamic hormones during sleep (Akerstedt 1984) could convey controlling signals from the circadian system to the sleepregulatory mechanisms of the brain stem. Receptors for peptide hormones have been localized to the putatively aminergic REM-off cell zones of the pons (Everitt, Hokfelt, Terenius, Tatemoto, Mutt \& Goldstein 1984; Glazer, Steinbusch, Verhofstad \& Basbaum 1981; Hokfelt, 
Hobson, Lydic \& Baghdoyan: Sleep cycle generation

Table 3. Predictions of the reciprocal-interaction model

\begin{tabular}{llll}
\hline Assumption of model & Prediction of model & Relevant experiment & Result and (conclusion) \\
\hline
\end{tabular}

A. Unit recording studies:

REM-on cells

(FTG, FTC, FTL, FTP, FTM) Fire selectively in REM

sleep

Firing rate increases before onset of REM sleep

Firing rate of FTG tonically modulated across sleep cycle

Bursts of FTG firing precede REMs

FTG cells tonically depolarize in REM

REM-off cells (LC/DRN/Pbl)

B. Dynamic manipulations: REM-on cells

(Putatively cholinergic/cholinoceptive) Lowest discharge rates in
REM sleep

REM-off cell groups are functionally similar

Periodic properties of DRN discharge related to sleep cycle rhythm

W modulated by firing of REM-off cells

Cholinergic agonists produce a REM sleep-like-state

REM sleep enhancement by cholinergic agonists is specific to PRF

Pharmacological induction of a REM-sleep-like state alters REM-on cell discharge
Record in free-moving animals (Siegel et al. 1977; Vertes 1977)

Record FTG across sleep cycle (Hobson, McCarley, Pivik \& Freedman 1974; Hobson, McCarley, Freedman \& Pivik 1974)

Record across sleep cycle (McCarley \& Hobson 1975)

Record FTG (ec) (Pivik et al. 1977)

Record (ic) across sleep cycle (Ito \& McCarley 1984)

Record (ec) across sleep cycle (McGinty \& Harper 1976; Hobson, McCarley \& Nelson 1983)

Compare time course of DRN and LC discharge across sleep cycle (Lydic et al. 1983a)

Spectral analyses of DRN discharge and behavioral state (Lydic et al. 1984)

Temporal analyses of DRN discharge at onset of $W$ (Lydic et al. 1985a)

Carbachol microinjection into PRF (Amatruda et al. 1975)

Bethanechol microinjection into PRF (Hobson, Goldberg et al. 1983)

Carbachol microinjection into midbrain, pontine and medullary RF (Baghdoyan, Rodrigo-Angulo et al. 1984)

Microinject carbachol into FTG and record REM-on cell activity in contralateral FTG (McKenna et al. 1975)
FTG firing not selective (FTG not exclusive REM sleep generator) Prediction confirmed (Supports role of FTG as part of REM sleep generator)

Prediction confirmed (FTG part of REM sleep generator)

Prediction confirmed (FTG activation could drive REMs)

FTG membrane excitability increased in REM sleep (Supports role of FTG as part of REM sleep generator)

MA neuron discharge negatively correlated with REM sleep (Supports permissive role for MA neurons in modulation of REM sleep) DRN profiles fit mathematically predicted functions derived from $\mathrm{LC}$ (Off-cell equations generically applicable)

DRN discharge rhythm phase-locked to ultradian sleep cycle (Temporal organization of sleep modulated by DRN discharge)

DRN discharge phase-leads onset of W (5-HT modulation of arousal)

Prediction confirmed (REM sleep generated by cholinoceptive PRF)

Prediction confirmed (Muscarinic cholinergic receptor involved in REM sleep generation) REM sleep decreased except from PRF (Midbrain and medullary RF not involved in initiation of REM sleep)

REM-on cell firing rates during carbachol-induced REM-sleep-like state were equal to levels during phys- 
Hobson, Lydic \& Baghdoyan: Sleep cycle generation

Table 3. (Continued)

\begin{tabular}{|c|c|c|c|}
\hline Assumption of model & Prediction of model & Relevant experiment & Result and (conclusion) \\
\hline & & & $\begin{array}{l}\text { iological REM sleep (FTG } \\
\text { REM-on cells mediate car- } \\
\text { bachol induced REM-sleep- } \\
\text { like state }\end{array}$ \\
\hline & $\begin{array}{l}\text { Pharmacological induction of } \\
\text { REM sleep signs alters } \\
\text { REM-on cell activity }\end{array}$ & $\begin{array}{l}\text { Inject eserine i.v. into de- } \\
\text { cerebrate cat and record } \\
\text { activity of PRF neurons } \\
\text { (Hoshino \& Pompeiano } \\
\text { 1976; Pompeiano \& Hoshino } \\
\text { 1976) }\end{array}$ & $\begin{array}{l}\text { PRF neurons showed mark- } \\
\text { ed increase in firing before } \\
\text { and during postural atonia } \\
\text { (PRF neurons may be in- } \\
\text { volved in generation of } \\
\text { postural atonia) }\end{array}$ \\
\hline & $\begin{array}{l}\text { Acetlycholinesterase inhibi- } \\
\text { tion produces a REM-sleep- } \\
\text { like state }\end{array}$ & $\begin{array}{l}\text { Neostigmine microinjection } \\
\text { into PRF (Baghdoyan, Mon- } \\
\text { aco et al. 1984) }\end{array}$ & $\begin{array}{l}\text { Prediction confirmed } \\
\text { (Endogenously released } \\
\text { ACh can trigger and main- } \\
\text { tain REM sleep) }\end{array}$ \\
\hline & $\begin{array}{l}\text { Cholinergic antagonists in- } \\
\text { hibit REM sleep }\end{array}$ & $\begin{array}{l}\text { Scopolamine microinjection } \\
\text { into PRF (Shiromani \& } \\
\text { McGinty 1983) }\end{array}$ & $\begin{array}{l}\text { Blocked carbachol-induced } \\
\text { REM-sleep-like state (Mus- } \\
\text { carinic cholinergic receptor } \\
\text { involved in REM sleep } \\
\text { generation) }\end{array}$ \\
\hline & & $\begin{array}{l}\text { Atropine microinjection into } \\
\text { PRF (Baghdoyan, Monaco } \\
\text { et al. 1984) }\end{array}$ & $\begin{array}{l}\text { Blocked neostigmine-in- } \\
\text { duced REM-sleep-like state } \\
\text { (Muscarinic cholinergic re- } \\
\text { ceptor involved in REM } \\
\text { sleep generation) }\end{array}$ \\
\hline & $\begin{array}{l}\text { Destruction of FTG elimi- } \\
\text { nates REM sleep }\end{array}$ & $\begin{array}{l}\text { Kainic acid lesion of FTG } \\
\text { (Sastre et al. 1981) }\end{array}$ & $\begin{array}{l}\text { REM sleep not abolished } \\
\text { (FTG not essential for REM } \\
\text { sleep generation) }\end{array}$ \\
\hline \multirow{9}{*}{$\begin{array}{l}\text { REM-off cells (Putatively } \\
\text { monoaminergic/cholinocep } \\
\text { tive) }\end{array}$} & $\begin{array}{l}\text { Activation of DRN de- } \\
\text { creases REM sleep }\end{array}$ & $\begin{array}{l}\text { Microinjection of } 5-\mathrm{HT} \text { an- } \\
\text { tagonists into DRN }\end{array}$ & No evidence found \\
\hline & & $\begin{array}{l}\text { Microinjection of cholinergic } \\
\text { agonists into DRN }\end{array}$ & No evidence found \\
\hline & $\begin{array}{l}\text { Activation of LC decreases } \\
\text { REM sleep }\end{array}$ & $\begin{array}{l}\text { Microinjection of NE antag- } \\
\text { onists into LC }\end{array}$ & No evidence found \\
\hline & & $\begin{array}{l}\text { Perfuse LC with ACh (Mas- } \\
\text { serano \& King 1982a) }\end{array}$ & $\begin{array}{l}\text { Prediction confirmed (LC } \\
\text { activation inhibits REM } \\
\text { sleep) }\end{array}$ \\
\hline & $\begin{array}{l}\text { Inactivation of DRN en- } \\
\text { hances REM sleep }\end{array}$ & $\begin{array}{l}\text { Cold block of DRN } \\
\text { (Cespuglio et al. 1981) }\end{array}$ & $\begin{array}{l}\text { Cooling induced REM sleep } \\
\text { (Supports permissive role of } \\
\text { DRN in REM sleep } \\
\text { modulation) }\end{array}$ \\
\hline & & $\begin{array}{l}\text { Microinjection of 5-HT ago- } \\
\text { nists into DRN }\end{array}$ & No evidence found \\
\hline & $\begin{array}{l}\text { Inactivation of LC enhances } \\
\text { REM sleep }\end{array}$ & $\begin{array}{l}\text { Cold block of LC (Cespuglio } \\
\text { et al. 1982) }\end{array}$ & $\begin{array}{l}\text { Cooling induced REM sleep } \\
\text { (Supports permissive role of } \\
\text { LC in REM sleep } \\
\text { modulation) }\end{array}$ \\
\hline & & $\begin{array}{l}\text { Microinjection of NE ago- } \\
\text { nists into LC (Masserano \& } \\
\text { King 1982b) }\end{array}$ & $\begin{array}{l}\text { Phentolamine in LC in- } \\
\text { creased REM sleep; } \\
\text { epinephrine in LC de- } \\
\text { creased REM sleep (Does } \\
\text { not support assumptions of } \\
\text { model) }\end{array}$ \\
\hline & $\begin{array}{l}\text { Pharmacological induction of } \\
\text { a REM-sleep-like state de- } \\
\text { creases DRN/LC discharge }\end{array}$ & $\begin{array}{l}\text { Microinject carbachol into } \\
\text { PRF and record DRN } \\
\text { (Steinfels et al. 1983) }\end{array}$ & $\begin{array}{l}\text { DRN activity reduced below } \\
\text { W and S levels during car- } \\
\text { bachol induced REM-sleep- } \\
\text { like state (Cessation of DRN } \\
\text { discharge modulates REM } \\
\text { sleep) }\end{array}$ \\
\hline
\end{tabular}


Hobson, Lydic \& Baghdoyan: Sleep cycle generation

Table 3. (Continued)

\begin{tabular}{|c|c|c|c|}
\hline Assumption of model & Prediction of model & Relevant experiment & Result and (conclusion) \\
\hline \multicolumn{4}{|l|}{$\begin{array}{l}\text { C. Postulated synaptic rela- } \\
\text { tionships underlying } \\
\text { reciprocal interaction: }\end{array}$} \\
\hline FTG-FTG & Excitatory & $\begin{array}{l}\text { Record (ic) FTG and stimu- } \\
\text { late contralateral FTG (Ito } \\
\text { \& McCarley 1984) }\end{array}$ & $\begin{array}{l}\text { Recorded monosynaptic } \\
\text { EPSPs in response to stim- } \\
\text { ulation (Supports concept of } \\
\text { autoexcitation within REM- } \\
\text { on cell population) }\end{array}$ \\
\hline FTG-LC & Excitatory & $\begin{array}{l}\text { Record LC and stimulate } \\
\text { FTG }\end{array}$ & No evidence found \\
\hline FTG-DRN & Excitatory & $\begin{array}{l}\text { Record (ec) DRN and stimu- } \\
\text { late FTG (Lydic et al. } \\
\text { 1983b) }\end{array}$ & $\begin{array}{l}\text { Long-latency, state-specific } \\
\text { excitation (Monosynaptic ex- } \\
\text { citatory projection not } \\
\text { demonstrated) }\end{array}$ \\
\hline LC-FTG & Inhibitory & $\begin{array}{l}\text { Record FTG and stimulate } \\
\text { LC }\end{array}$ & No evidence found \\
\hline LC-LC & Inhibitory & $\begin{array}{l}\text { Record LC during anti- } \\
\text { dromic activation of LC } \\
\text { (Aghajanian et al. 1977) }\end{array}$ & $\begin{array}{l}\text { Stimulation of dorsal NE } \\
\text { bundle suppressed LC firing } \\
\text { (Supports concept of auto- } \\
\text { inhibition within REM-off } \\
\text { cell population) }\end{array}$ \\
\hline LC-DRN & Inhibitory & $\begin{array}{l}\text { Record DRN and stimulate } \\
\text { LC }\end{array}$ & No evidence found \\
\hline DRN-FTG & Inhibitory & $\begin{array}{l}\text { Record FTG and stimulate } \\
\text { DRN (Nakamura 1975) }\end{array}$ & $\begin{array}{l}\text { FTG neurons inhibited } \\
\text { (Supports postulated inhibi- } \\
\text { tion of REM-on cells by } \\
\text { REM-off cells) }\end{array}$ \\
\hline DRN-LC & Inhibitory & $\begin{array}{l}\text { Record LC and stimulate } \\
\text { DRN (Segal 1979) }\end{array}$ & $\begin{array}{l}\text { Responsiveness of LC neu- } \\
\text { rons inhibited (Supports } \\
\text { concept of inhibitory con- } \\
\text { nections between REM-off } \\
\text { cell groups) }\end{array}$ \\
\hline DRN-DRN & Inhibitory & $\begin{array}{l}\text { Record DRN during anti- } \\
\text { dromic activation of DRN } \\
\text { (Wang \& Aghajanian 1977b) }\end{array}$ & $\begin{array}{l}\text { Stimulation of ventromedial } \\
\text { tegmentum suppressed } \\
\text { DRN firing (Supports con- } \\
\text { cept of autoinhibition within } \\
\text { REM-off cell population) }\end{array}$ \\
\hline \multicolumn{4}{|l|}{$\begin{array}{l}\text { D. Neurotransmitter identi- } \\
\text { fication and effects: } \\
\text { REM-on cells }\end{array}$} \\
\hline \multirow[t]{4}{*}{ (FTG, FTC, FTL, FTP, FTM) } & Cholinergic & $\begin{array}{l}\text { Immunohistochemical local- } \\
\text { ization of ChAT containing } \\
\text { neurons in PRF (Kimura et } \\
\text { al. 1981) } \\
\text { Immunohistochemical local- } \\
\text { ization of ChAT in PGO } \\
\text { burst cell zone (Jones 1985a; } \\
\text { Sakai 1985c) }\end{array}$ & $\begin{array}{l}\text { Pontine FTG neurons ChAT } \\
\text { positive (Supports postu- } \\
\text { lated cholinergic identity of } \\
\text { REM-on cells) } \\
\text { ChAT positive neurons in } \\
\text { PGO burst cell zone (Sug- } \\
\text { gests cholinergic identity of } \\
\text { REM-on cells) }\end{array}$ \\
\hline & Excitation by $\mathrm{ACh}$ & $\begin{array}{l}\text { Simultaneous cholinergic } \\
\text { stimulation and recording of } \\
\text { same REM-on cells }\end{array}$ & No evidence found \\
\hline & Inhibition by $5-\mathrm{HT}$ & $\begin{array}{l}\text { Simultaneous serotonergic } \\
\text { stimulation and recording of } \\
\text { same REM-on cells }\end{array}$ & No evidence found \\
\hline & Inhibition by NE & $\begin{array}{l}\text { Simultaneous noradrenergic } \\
\text { stimulation and recording of } \\
\text { same REM-on cells }\end{array}$ & No evidence found \\
\hline
\end{tabular}

(continued) 
Hobson, Lydic \& Baghdoyan: Sleep cycle generation

Table 3. (Continued)

\begin{tabular}{llll}
\hline \hline Assumption of model & Prediction of model & Relevant experiment
\end{tabular}

REM-off cells

(LC)

Noradrenergic*

Excitation by ACh

Inhibition by $5-\mathrm{HT}$

Inhibition by $\mathrm{NE}$

REM-off cells

(DRN)

Serotonergic*

Excitation by ACh

Inhibition by $5-\mathrm{HT}$

Inhibition by $\mathrm{NE}$
Histofluorescent localization of NE in LC (Dahlstrom \& Fuxe 1965)

Simultaneous cholinergic stimulation and recording of LC cells (Guyenet \& Aghajanian 1979)

Simultaneous serotonergic stimulation and recording of LC cells

Stimulation of DRN and simultaneous recording of LC cells (Segal 1979)

Simultaneous noradrenergic stimulation and recording of LC cells (Cederbaum \& Aghajanian 1977)

Histofluorescent localization of 5-HT in DRN (Dahlstrom \& Fuxe 1965)

Simultaneous cholinergic stimulation and recording of DRN cells

Simultaneous serotonergic stimulation and recording of DRN cells (Haigler \& Aghajanian 1974)

Simultaneous noradrenergic stimulation and recording of DRN cells (Baraban \& Aghajanian 1980)
LN neurons positive for NE (Consistent with postulated noradrenergic nature of REM-off cells) Iontophoretic application of ACh excited LC neurons (Consistent with hypothesized excitatory input from REM-on to REM-off cells)

No evidence found

Responsiveness of LC neurons inhibited (Supports 5-HT mediated inhibition of LC neurons)

Iontophoresis of NE inhibits LC discharge (Supports concept of autoinhibition within REM-off cell population)

DRN neurons positive for 5-HT (Consistent with postulated serotonergic nature of REM-off cells)

No evidence found

Iontophoresis of 5-HT inhibits firing of DRN neurons (Supports concept of autoinhibition within REM-off cell population) Iontophoresis of NE at low doses activates DRN cells (Does not support proposed inhibitory connections between REM-off cell groups)

*Indicates studies upon which neurotransmitter identity tenets of the original reciprocal-interaction model were based.

Note: Summary of data supporting and not supporting predictions derived from the reciprocal-interaction model. The table summarizes predictions in four areas of investigation: (A) unit recording studies of REM-on and REM-off cells; (B) dynamic manipulations of neuronal systems involved in sleep cycle control; (C) postulated synaptic relationships between REM-on and REM-off cells; and (D) histochemical identity and neurotransmitter effects on cellular components of the reciprocal-interaction model.

Abbreviations: $\mathrm{ec}=$ extracellular recording; ic $=$ intracellular recording; FTG = gigantocellular tegmental field; FTC $=$ central tegmental field; FTL = lateral tegmental field; FTP = pontine parvocellular tegmental field; FTM = magnocellular tegmental field; LC = locus coeruleus; $\mathrm{DRN}=$ dorsal raphe nucleus; $\mathrm{Pbl}=$ peribrachial region; 5 - $\mathrm{HT}=$ serotonin; $\mathrm{ACh}=$ acetylcholine; $\mathrm{NE}$ $=$ norepinephrine; ChAT $=$ choline acetyltransferase; $\mathrm{MA}=$ monoaminergic; $\mathrm{PRF}=$ pontine reticular formation; $\mathrm{RF}=$ reticular formation; $\mathrm{W}=$ waking.

Ljungdahl, Steinbusch, Verhofstad, Nilsson, Brodin, Pernow \& Goldstein 1978; Leger, Wiklund, Descarries \& Person 1979; Moss, Glazer \& Basbaum 1981; Vincent, Satoh, Armstrong \& Fibiger 1983). Progress in research on humoral factors influencing the ultradian sleep cycle and circadian rhythms has recently been reviewed (Inoue, Honda \& Komoda 1985).

The foregoing data suggest a number of testable hypotheses about possible mechanisms that may mediate circadian influences on the ultradian sleep-wake cycle. 
For example, during the activity phase of the circadian cycle the REM-on and REM-off neurons of the pons may be influenced by peptide hormones of hypothalamic origin. In the cat it has been demonstrated that cerebrospinal fluid levels of vasopressin exhibit a largeamplitude daily rhythm, with peak concentrations occurring during the light phase of the circadian cycle (Reppert, Artman, Swaminathan \& Fisher 1981). However, in the rat (which is active at night), multiple-unit activity recorded from the SCN was shown to have a daytime maximum discharge pattern (Inouye \& Kawamura 1979), and explants of rat $S \mathrm{CN}$ have recently been shown to exhibit a circadian release of vasopressin with maximal levels occurring during the subjective day (Earnest \& Sladek 1984). Cellular discharge data are needed to advance these ideas about the interactions between circadian and ultradian rhythms from the level of speculation to the level of hypothesis testing. It should be clear from the previous discussion of pharmacological experiments (Part IV) that hypotheses about the role of peptides in regulating the interaction between the sleep cycle and circadian rhythms are also testable with the chemical microinjection method.

\section{E. Clinical applications of the reciprocal-interaction model}

The past decade has witnessed an explosion of research on disorders of human sleep. Studies of the physiological basis of sleep cycle control provide a major vehicle for understanding the pathogenesis of these myriad disorders. This relationship between neurobiology and sleepdisorders medicine has been discussed in detail (Hobson 1983a), showing that the study and treatment of clinical sleep disorders constitutes an important arena for application and testing of the reciprocal-interaction model (Table 4). Although hardly as rigorous as the laboratory, the clinic can thus provide still another proving ground for concepts derived from basic research in neurobiology.

For example, considerable evidence suggests an overlap between neurobiological mechanisms involved in depression and neural mechanisms contributing to the control of sleep (McCarley 1982). Certain types of depressive disorders have been proposed to result from increased ratios of cholinergic to adrenergic activity (Janowsky, El-Yousef, Davis \& Sederke 1972), and pharmacological models of sleep cycle control had proposed a role for cholinergic and monoaminergic systems (Karczmar et al. 1970) long before the supporting neurophysiological evidence became available. A role for cholinergic mechanisms in sleep and depression has been demonstrated in clinical studies (Gillin, Sitaram, Janowsky, Risch, Huey \& Storch 1982; Sitaram, Nurnberger, Gershon \& Gillin 1982; Sitaram, Wyatt, Dawson \& Gillin 1976). These findings are clearly compatible with the pharmacological studies described in Part IV of this paper and consonant with the predictions of the reciprocal-interaction model outlined in Table 3.

To help organize and understand the specific details given in Table 4, we have derived three general pathophysiological principles from the reciprocal-interaction model:
Hobson, Lydic \& Baghdoyan: Sleep cycle generation

1. The coupled oscillator concept. The brain contains many oscillating neuronal systems that display highly variable time constants. The hypothalamic SCN has been shown to play a role in the generation of circadian (approximately $24 \mathrm{hr}$ ) rhythms; the pontine brain stem has been shown to play a role in generating the ultradian (period length $\ll 24 \mathrm{hr}$ ) sleep cycle, and key components of the respiratory rhythm generator (period length $<1$ $\mathrm{min}$ ) reside in the medullary brain stem. These three oscillatory systems are known to be anatomically coupled and strongly interactive. This interaction is illustrated by single-unit recordings during sleep and wakefulness from the pneumotaxic center of the dorsolateral pontine tegmentum. These recordings have revealed the presence of PGO burst cells (McCarley, Nelson \& Hobson 1978), REM-off cells (Hobson, McCarley \& Nelson 1983), and respiratory neurons (Lydic \& Orem 1979).

The generalizable result of such functional interaction is oscillator coupling: Circadian rhythms, the ultradian sleep cycle, and respiration covary dynamically and with abounding dysfunctional possibilities. For example, circadian phase shifts of the sleep-wake cycle have been related to certain depressive disorders (Wehr, WirzJustice, Goodwin, Duncan \& Gillin 1979), and the onset and duration of sleep are known to be dependent on the circadian rhythm of core body temperature (Czeisler, Weitzman, Moore-Ede, Zimmerman \& Knauer 1980; Zulley, Wever \& Aschoff 1981). In REM sleep, respiratory neurons in the peribrachial region of the pons display a reduced discharge frequency (Lydic \& Orem 1979), and disorders of respiratory control during sleep are believed to underlie a variety of clinical problems such as the sudden infant death syndrome, the Pickwickian syndrome, and the sleep apneas (Phillipson 1978).

2. The neurotransmitter ratio concept. With regard to the pontine system implicated in the generation of the ultradian sleep cycle, consider the consequences of a change in the ratio of activity of the putatively interacting aminergic and cholinergic populations. According to the reciprocal-interaction model, if the net activity level of aminergic neurons is increased (or if the activity of cholinergic neurons is decreased) the result will be increased wakefulness. In clinical terms this can be manifested as insomnia. That such a concept is valid is supported by the known association of insomnia with stress, whose effects are mediated, in part, by central sympathetic activation. By contrast, if there is a decline in aminergic (or an increase in cholinergic) neurotransmission the net result will favor the appearance of sleep. If this occurs at an inappropriate phase of the circadian day the result will be hypersomnia or excessive daytime sleepiness. This idea gains further credence from the observation that the hypersomnias of both depression and narcolepsy are reversed by pharmacological agents that facilitate aminergic neurotransmission (see Table 4).

3. Desynchronization of distributed neuronal populations. Consider the consequences of activating the multiplicity of distinct neuronal subpopulations known to be involved in generating REM sleep phenomena (Figures 1 and 5). A precise temporal coordination is required if, for example, the cortex and spinal cord are to undergo 
Hobson, Lydic \& Baghdoyan: Sleep cycle generation

Table 4. Some data from sleep-disorders medicine of possible relevance to testing the reciprocal-interaction model in man

\begin{tabular}{|c|c|c|}
\hline Sleep disorder & Clinical phenomenon & $\begin{array}{l}\text { Interpretation with regard to the } \\
\text { reciprocal-interaction model }\end{array}$ \\
\hline \multirow[t]{6}{*}{ Narcolepsy } & $\begin{array}{l}\text { Sleep attacks, cataplexy, hypnagogic hallucina- } \\
\text { tions, sleep paralysis }\end{array}$ & $\begin{array}{l}\text { Reciprocal-interaction model may provide inter- } \\
\text { pretative framework for understanding clinical } \\
\text { phenomena }\end{array}$ \\
\hline & Symptoms improved by: & \\
\hline & Sympathomimetic amines & $\begin{array}{l}\text { Consistent with aminergic tenet of reciprocal-in- } \\
\text { teraction model }\end{array}$ \\
\hline & Tricyclic antidepressants & $\begin{array}{l}\text { Consistent with aminergic and cholinergic tenets } \\
\text { of reciprocal-interaction model }\end{array}$ \\
\hline & Canine model of narcolepsy & $\begin{array}{l}\text { Experimental tests of reciprocal-interaction model } \\
\text { in dogs may elucidate pathophysiology of } \\
\text { narcolepsy }\end{array}$ \\
\hline & Cataplectic attacks provoked by i.v. eserine & $\begin{array}{l}\text { Consistent with cholinergic tenet of reciprocal-in- } \\
\text { teraction model }\end{array}$ \\
\hline \multirow[t]{4}{*}{ Depression } & Hypersomnia, decreased REM latency & $\begin{array}{l}\text { Suggests weakened aminergic inhibition and/or } \\
\text { increased cholinergic excitation of REM sleep } \\
\text { generator }\end{array}$ \\
\hline & Symptoms reversed by: & \\
\hline & Amphetamines & $\begin{array}{l}\text { Consistent with aminergic tenet of reciprocal-in- } \\
\text { teraction model }\end{array}$ \\
\hline & Tricyclic antidepressants & $\begin{array}{l}\text { Consistent with aminergic and cholinergic tenets } \\
\text { of reciprocal-interaction model }\end{array}$ \\
\hline \multirow[t]{5}{*}{ Insomnia } & Stress-related & $\begin{array}{l}\text { Suggests mediation by facilitation of central } \\
\text { aminergic neuronal systems }\end{array}$ \\
\hline & $\begin{array}{l}\text { Associated with anxiety, obsessional thinking, and } \\
\text { increased muscle tension }\end{array}$ & $\begin{array}{l}\text { Suggests facilitation of central aminergic neurons } \\
\text { via corticopontine and spinopontine influences }\end{array}$ \\
\hline & & $\begin{array}{l}\text { Both could be mediated by observed increases in } \\
\text { aminergic and midbrain reticular discharge in } \\
\text { arousal }\end{array}$ \\
\hline & Symptoms improved by: & \\
\hline & Benzodiazepines & $\begin{array}{l}\text { Known suppression of } 5-\mathrm{HT} \text { cells via GABA-ergic } \\
\text { mechanisms consistent with serotonergic tenet of } \\
\text { the reciprocal-interaction model }\end{array}$ \\
\hline \multirow[t]{4}{*}{$\begin{array}{l}\text { Sleep Apnea } \\
\text { Syndromes }\end{array}$} & Apnea at sleep onset (central component) & $\begin{array}{l}\text { Suggests loss of facilitatory drive on respiratory } \\
\text { oscillator }\end{array}$ \\
\hline & Obstruction at sleep onset (peripheral component) & Suggests loss of airway muscle tone \\
\hline & & $\begin{array}{l}\text { Both could be mediated by observed decreases in } \\
\text { reticular and aminergic neuronal discharge at } \\
\text { sleep onset }\end{array}$ \\
\hline & Apnea during REM sleep & $\begin{array}{l}\text { Consistent with observed decreases in tonic and } \\
\text { phasic activity of reticular formation and respira- } \\
\text { tory neurons during REM sleep }\end{array}$ \\
\hline $\begin{array}{l}\text { Sleep Walking and } \\
\text { Talking }\end{array}$ & $\begin{array}{l}\text { Emergence of organized motor acts in Stage IV of } \\
\text { NREM sleep }\end{array}$ & $\begin{array}{l}\text { Suggests dissociation of activation of motor pat- } \\
\text { tern generators from activation of cerebral cortex } \\
\text { Consistent with observed progressive activation of } \\
\text { pontine reticular neurons in NREM sleep with } \\
\text { long phase lead over cortical activation }\end{array}$ \\
\hline Enuresis & $\begin{array}{l}\text { Failure to arouse in response to internally gener- } \\
\text { ated signals from bladder especially in NREM } \\
\text { sleep }\end{array}$ & $\begin{array}{l}\text { Consistent with above observations for sleep } \\
\text { walking and talking; also decreasing aminergic } \\
\text { and increasing cholinergic drive on bladder mus- } \\
\text { culature in sleep is consistent with aminergic and } \\
\text { cholinergic tenets of reciprocal-interaction model } \\
\text { at peripheral as well as central levels }\end{array}$ \\
\hline
\end{tabular}


simultaneous excitability shifts but in different directions (excitation and inhibition, respectively). The functionally opposed nature of these excitability changes makes temporal organization even more critical, and disruptions of temporal organization may explain a number of clinical disorders (Table 4). In REM sleep, central motor pattern generators are excited while final common path motoneurons are inhibited. In cases where normal temporal organization is disrupted, inadvertent motor acts can occur during sleep (as with sleepwalking), or mental states appropriate only to sleep can occur in waking (as with the hypnagogic hallucinations of narcolepsy).

Table 4 applies these concepts of sleep pathophysiology to specific disorders and gives several examples where the accuracy of therapeutic prediction strengthens the explanatory value of the reciprocal-interaction model. The model can thus be used to generate a therapeutic rationale based on cellular and molecular data for treating a number of sleep disorders.

As one particularly instructive example, consider the pathogenesis of the clinical disorder of narcolepsy and the predictiveness derived from the reciprocal-interaction model. It has been experimentally demonstrated that dogs with narcolepsy show a significant increase in cataplexy after cholinergic stimulation (Delashaw, Foutz, Guilleminault \& Dement 1979). Symptomatic improvement of narcolepsy may be induced by aminergic agonists (e.g., amphetamine), by amine reuptake blockers (e.g., the tricyclics), or by preventing enzymatic degradation of the biogenic amines (e.g., amine oxidase inhibition). Furthermore, detailed histochemical analyses of the brains of narcoleptic dogs directly supported the prediction of the reciprocal-interaction model that the initiation and maintenance of REM sleep is linked to a critical balance between serotonergic, adrenergic, and cholinergic neuronal networks in the pontine brain stem (Mefford, Baker, Boehme, Foutz, Ciaranello, Barchas \& Dement 1983).

The growing field of sleep-disorders medicine can be expected to mature if it becomes more deeply rooted in cellular and molecular neuroscience. The demonstrated explanatory value and predictive powers of the reciprocal-interaction model have led some of its proponents to recognize its clinical utility, and, as befits the growth of science, the clinical data have, in turn, served to validate the reciprocal-interaction model.

\section{Summary and conclusions}

1. This paper has discussed the evidence that has accumulated during the past 10 years concerning the neural regulation of the mammalian sleep cycle. The discussion has focused on revisions of the reciprocal-interaction model and an evaluation of its continued heuristic and empirical value. The model has been modified to accord with the new evidence, and additional criteria for accepting or rejecting causal hypotheses concerning the neural regulation of the ultradian sleep cycle have been elaborated.

2. One major goal has been to document the shift in conceptual emphasis from localized sleep centers (be they nuclei or specific cellular groups) to interacting neuronal populations. In sharp contrast to the original view of the putative regulatory neurons as few and highly localized, their multiple, distributed, and interpenetrated nature is now appreciated.

3. Our revised concept of the putative sleep cycle oscillator as consisting of anatomically distributed and neurochemically interpenetrated populations of cells has strong implications for experimental strategies in sleep research and related fields of mammalian behavioral neurobiology:

(a) The failure to disrupt a behavior by localized lesions to any one nuclear structure does not conclusively rule out participation of that nucleus in the normal generation of physiological or behavioral events.

(b) The neurotoxic destruction of a single cholinergic or aminergic cell type may not abolish REM sleep because of the multiplicity of neurotransmitter systems involved in physiological modulation of the REM-on populations.

(c) The dissociations of cellular discharge patterns from the behaviors with which they are normally correlated after lesions cannot be taken as definitive evidence against the participation of those cells in behavioral state regulation under physiological conditions.

(d) The correlation of a neuronal discharge pattern with a specific behavioral or physiological event does not exclude a significant role for that cell group in REM sleep regulation.

4. A second major goal of this paper has been to illustrate that synthetic and integrative schemata, such as the reciprocal-interaction model of sleep cycle control, may have an explanatory and heuristic value that is lacking in approaches which do not go beyond asserting that a given nucleus, cell type, or a neurotransmitter is, or is not, involved in sleep cycle regulation. Despite differences in detail and despite apparent controversy, we find widespread agreement concerning the data base and major conceptual aspects of the model.

5. To resolve interpretative issues and to test the many predictions of the model that we have specified, we see a need for new methods that allow causal interpretation rather than mere correlational observation. In this respect, we see sleep research as having come of age experimentally. Among efforts to enhance REM sleep, the recent experimental evidence reviewed here illustrates the value of the chemical microinjection technique. It has long been appreciated that electrical stimulation may give misleading results due to the well-known joint stimulation of fibers of passage. The concept that REM sleep control is strongly influenced by anatomically distributed and neurochemically interpenetrated neuronal populations clearly means that electrical stimulation will simultaneously activate REM-on (putatively cholinergic) and REM-off (putatively aminergic) cells. In contrast, the chemical microinjection method allows the investigator to activate, at will, specific receptors of discrete neuronal populations and to observe the effects on the sleep cycle. This method has proved its usefulness and deserves extensive exploitation.

6 . We see the future as also providing opportunities to study the implication of the data base and reciprocalinteraction model for circadian-ultradian interactions and for sleep-disorders medicine. 
7. On one point there can be no disagreement: It has been unequivocally demonstrated that sleep cycle regulation is far more complicated than it was thought to be 10 years ago. Generation of the ultradian sleep cycle cannot be accounted for on the basis of any single nuclear group or any single neurotransmitter. Now that many of the obstacles to the long-term recording of cells have been overcome, and now that the excitability of chemically related neuronal populations can be experimentally controlled, the sleep cycle provides a unique opportunity to study the cellular mechanisms of mammalian central pattern generation and the endogenous alterations in neuronal excitability that underlie changes in behavioral state.

\section{ACKNOWLEDGMENTS}

This work was supported by National Institute of Mental Health grant MH-13923 (J.A.H.), National Research Service Award MH-14275 (H.A.B. and R.L.), and an award to R.L. from the William F. Milton Fund of Harvard Medical School.

\section{Open Peer Commentary}

Commentaries submitted by the qualified professional readership of this journal will be considered for publication in a later issue as Continuing Commentary on this article. Integrative overviews and syntheses are especially encouraged.

\section{Rapid eye movements and the cerebellum}

\section{John Antrobus \\ Department of Psychology, The City College of the City University of New York, New York, N.Y. 10031}

This most welcome revision of the 1975 reciprocal-interaction model of REM sleep will unquestionably provide an organizing reference point for sleep research throughout the coming decade. The exponentially increasing complexity of biological sleep research over the past three decades and the identification of increasingly complex interactions occurring within and between neuronal units and within and across temporally distributed biological states - to all of which this revised model is responsive - suggest that the next generation of models must be built in such a way that the simultaneous interaction of all of the component parts can be simulated with some precision. We need to know, for example, whether a particular model generates REM/NREM cycles of 9 or $90 \mathrm{~min}$. This means that the interacting neural components must be represented in a computer model in which the parameters of the component parts are quantitatively specified. Until such models are constructed, the increased theoretical power of this neuronal-populations reciprocal-interaction model will make decisive tests of the model elusive. Correspondingly, empirical 'evaluation of the model will require an increased order of experimental design complexity. The neuronal-populations model has so many degrees of freedom that only multivariate studies can provide appropriate tests of the multiply interacting components. It is to be hoped that this new model will stimulate both the multivariate and the parametric research that will make quantitative simulations possible within the next decade. In this regard, it would have been helpful to see an expanded discussion of the quantitative model examined by McCarley and Massaquoi (1985) inasmuch as the work is in press.

As the review suggests, the functional role of phasic events in the REM/NREM cycle remains ambiguous. A consideration of the potential role of the cerebellar cortex in the generation of REM sleep eye movements (EMs) may help to clarify the function of EMs and the dramatic high-frequency pontogeniculo occipital (PGO) activity by placing them in the context of an integrated system for the production of waking saccades. Specifically, it is the duration of firing of the burst cells of the cerebellar cortex that determines the magnitude and velocity of saccadic EMs in the waking state. The pathway from the cerebellar burst cells to the oculomotor neurons passes through the brachium conjunctivum, where Nelson, McCarley, and Hobson (1983) have identified the burst cells associated with REM sleep EMs. Is it possible that the activity of these REM burst cells originates in the cerebellum? Ito (1975) has described the vestibular-cerebellar-ocular network by which head movement vestibular information modifies the cerebellar burst cell firing rates. Extensive afferents to the cerebellum from the association cortex (Kornhuber 1975) and, in primates, the frontal eye fields (Crowne 1983), indicate pathways by which higher cognitive processes, including visual imagery and goal-related attention processes, modify waking saccades and may similarly modify sleep EMs when the frontal cortex is sufficiently activated as in REM sleep (i.e., the mesencephalic reticular formation). Although the burst cells of the cerebellar cortex are an essential component in the waking saccade system, they have never, to my knowledge, been studied in relation to REM PGO and EM activity.

The high firing rate of the oculomotor neurons (up to $600 \mathrm{~Hz}$ in the waking state), although dampened in REM sleep, may be determined more by the requirements of the waking visual information-processing system than by any unique REM sleep process. To meet the requirements of waking visual perception, the extraocular muscles are capable of very fast and precise movement. They contain no stretch receptors (which would dampen a movement response); so movement control is completely determined by the oculomotor neuron firing rates. These high rates should permit a high order of velocity-amplitude discrimination control. The absence of Renshaw cells in the oculomotor neuron pathways removes the neurons from spinal recurrent inhibition and thereby further restricts saccadic control to the burst cells. This absence of recurrent inhibition in the oculomotor neurons may account, in part, for the fact that only EMs are spared from the inhibitory spinally induced atonia that characterizes the rest of the motor system during REM sleep. A functional reason for the absence of this inhibitory control that characterizes other motor systems in REM may be that in order for saccadic movement in the waking state to be so fast, the capability of midsaccade modification based on midsaccade visual feedback must be sacrificed. Accordingly, the saccades are controlled by a feedforward process (Aschoff 1974). In sum, the entire system lacks the inhibitory mechanisms characteristic of the skeletal-motor system and therefore emerges as the most dramatically active REM sleep activity. But this dramatic characteristic may have misled us into attributing to the PGO-REM system a more important functional role in the REM/NREM cycle than it deserves.

Evidence for the role of the cerebellum in the programming of EMs in REM would suggest that the possible role of the cortex, both occipital and frontal, in the production of EMs should be reexamined. The neuronal-populations model places the origin of the EMs in the pons. Nelson, McCarley, and Hobson (1983) have suggested that the cortex receives this pons- 
initiated EM information but does not contribute to its production even though an occipital response precedes the EM (McCarley, Winkelman \& Duffy 1983; Monaco, Baghdoyan, Nelson \& Hobson 1984). This issue is an important one for neurocognitive models of the production of visual imagery in REM sleep. Do the eyes respond to some kind of visual scene analysis in the associative cortex or does the cortex create some kind of post hoc visual analysis to interpret the pons-elicited EMs? Given the multiple sources of waking and REM EM control, the answer will not be easy to determine.

In the course of comparing waking and sleep EM models it is important to note that smooth pursuit EMs are not programmed in the cerebellum, and they do not decussate at the same points as the saccade system (Aschoff 1974). In conclusion, although the inclusion of the cerebellar burst cells in the phasic portion of the model does not speak to the reciprocal-interaction feature of the neuronal-populations model, it may help to provide a more adequate context for understanding the relation between the phasic and tonic characteristics of REM sleep.

\section{Sleep homeostasis}

\author{
Alexander A. Borbély \\ Institute of Pharmacology, University of Zürich, $\mathrm{CH}-8006$ Zürich, \\ Switzerland
}

The reciprocal-interaction hypothesis has the great merit of specifying in mathematical terms how the interaction between two neuronal groups may give rise to the non-REM/REM sleep cycle. Because the neurobiological basis for the original hypothesis (McCarley \& Hobson 1975b) was clearly stated, its tenets could be verified in experimental studies. The emerging new data have made it necessary to revise some of the earlier assumptions, while maintaining the basic idea of a reciprocal interaction between neuronal systems. In the present modified and extended version of the model, Hobson, Lydic, and Baghdoyan do not restrict their analysis to the non-REM/REM sleep cycle per se but also examine the influence of other factors such as the circadian rhythm. This makes it possible to view the reciprocal-interaction hypothesis in a broader perspective. However, another fundamental aspect of sleep regulation, sleep homeostasis, has not yet been incorporated.

The notion of sleep homeostasis refers to an intrinsic tendency to maintain the duration and/or intensity of sleep within certain physiological limits. This tendency becomes evident when the sleep/wake ratio is experimentally altered. Thus, sleep deprivation gives rise to an intensification and/or prolongation of sleep, whereas excess sleep induces the opposite changes (see Borbély 1982 and Daan, Beersma \& Borbély 1984 for references). The notion of sleep intensity is largely based on the analysis of the progressive changes occurring within nonREM sleep. The recognition that non-REM sleep is itself a heterogenous state adds a level of complexity to Hobson et al.'s view that "mammalian sleep is a bistable process consisting of a continuous alternation between two different states." Consistent trends within non-REM sleep can be recognized from changes of EEG slow wave activity (SWA) as measured by spectral analysis. There is strong evidence that this non-REM parameter represents a sleep intensity index in both man (Borbély, Baumann, Brandeis, Strauch \& Lehmann 1981) and animals (Borbély, Tobler \& Hanagasioglu 1984). Thus SWA shows a declining trend throughout the sleep period and is enhanced by prolonged prior waking. If the occurrence of SW.A in nonREM sleep is prevented by sensory stimulation, a compensatory increase occurs during recovery sleep. The notion of sleep homeostasis is not restricted to non-REM sleep but encom- passes REM sleep as well. The enhancement of REM sleep in response to total sleep deprivation or selective REM sleep deprivation is well documented (see Borbély 1982).

The neurochemical basis of sleep homeostasis is unknown. In recent years evidence has accumulated indicating that endogenous sleep-promoting substances may be involved (see Inoue \& Borbély 1985 for an overview). In their target article, Hobson et al. focus upon cholinergic mechanisms, and on the interaction of cholinergic and aminergic systems, to account for features of the non-REM/REM cycle. However, there is little evidence to indicate that progressive changes in transmitter release or receptor sensitivity occur in the course of sleep deprivation. It therefore seems unlikely that the homeostatic aspect of sleep regulation can be explained exclusively on the basis of "classical" transmitter mechanisms. Jouvet (1984) has recently proposed that aminergic transmitters may act in a chain of biochemical events that lead to the elaboration of a specific "sleep factor."

As the title of the target article indicates, sleep research has recently witnessed an evolution of its conceptual framework. With their overview, Hobson, Lydic, and Baghdoyan have made a bold effort to integrate a large body of data. This commentary is intended as a reminder that parts of the "puzzle" are still disconnected. Nevertheless, a picture begins to emerge.

\section{Relationships between pontogeniculooccipital waves and ocular movements}

\author{
Raymond Cespuglio \\ Département de Médecine Expérimentale, Université Claude Bernard, \\ 69373 Lyon Cedex 08, France
}

The paper of Hobson, Lydic \& Baghdoyan is an important and exhaustive review discussing data accumulated during the past 10 years concerning the complex neuronal regulations underlying the mammalian sleep cycle.

The basic outline guiding the authors in the complex neuronal network involved in sleep consists of a putative sleep cycle oscillator with anatomically distributed and neurochemically interpenctrating populations of cells. This view contrasts with the concept of localized sleep centers in a structure or a group of structures.

Our comments specifically concern one of the main parameters that define sleep: the phasic events of paradoxical sleep (REM sleep). In section B of part II Hobson et al. write that when the eyes move to the right during REM sleep, the PGO waves of the right lateral geniculate body (LGB) and the right posterolateral cortex are larger in amplitude than those recorded from the left side of the brain. To record the horizontal eye movements (Monaco, Baghdoyan, Nelson \& Hobson 1984), electrooculogram leads made of stainless steel were placed in the zygomatic arch directly lateral to the orbits. The direction of eye movements was determined by direct observation of the eye with simultaneous observation of the direction of pen deflection on the polygraph. The time constant for eye movement recording was very short and estimated at about $200 \mathrm{~ms}$.

We have demonstrated a different "PGO wave-horizontal eye movement" relationship by simply recording the horizontal eye movements through the electromyographic activity of the lateral rectus (LR) muscles; these muscles are supplied by the abducens VI nerve and ensure movements of the eyeballs only in the horizontal plane. Our "PGO wave-horizontal eye movement" relationship is the following: A primary $P C O$ wave in one LGB (larger in amplitude than in the contralateral LGB) is 
accompanied by electromyographic "inhibition" of the ipsilateral LR muscle and by activation of the contralateral LR muscle. This relationship has been demonstrated both in reserpinized cats (Cespuglio, Verchere, Laurent \& Jouvet 1975; Cespuglio, Laurent \& Jouvet 1975) and also in "encephale isolé" cats exhibiting spontaneous episodes of REM sleep (Cespuglio, Laurent \& Calvo 1976).

The discrepancies between our results and those of Monaco et al. (1984) can be explained by the differences in method. The electrooculogram as the only criterion for horizontal eye movement is not precise enough; moreover, the pattern of PGO waves during REM sleep is much too complex to be analyzed directly without pitfalls. The reserpine PGO wave model that we first analyzed is simpler. In such a preparation, 90 minutes after injection of the drug, isolated PGO waves with two components (large and small amplitude, separated by an interval of 80 $\mathrm{ms}$ ) are recorded; these PGO waves are produced by the alternating activity ( $80 \mathrm{~ms}$ of delay) of the two pontine generators as shown by their relationships with the LR muscles (see above). During REM sleep the isolated PGO waves usually contain two components whose interval cannot be distinguished if one uses only polygraphic recordings. This fact is a source of error when correlating these REM sleep phasic events with the electrooculogram.

This difficulty is inherent in the REM sleep PGO model but can be overcome in the following way: It is now accepted that PGO waves are generated bilaterally in the pontine peribrachial area, whose cellular organization has been described (Sakai 1980). From this area both ipsi- and contralateral pathways impinging on the LGB have been described; the contralateral pathway crosses the midline through the supraoptic decussation (Laurent, Cespuglio \& Jouvet 1972; 1974). A transection of this decussation permits recording in each LGB PGO waves generated only in the ipsilateral side; under such conditions right LGB PGO waves (primary wave only, large amplitude) are correlated with left LR muscle activation and vice versa, either in reserpinized cats or in spontaneous REM sleep episodes occurring in "encephale isolé" cats (Cespuglio et al. 1976, Fig. 1).

From a more general point of view, it is clear that PGO waves have specific electrical characteristics as well as a topographically well defined anatomical substrate responsible for their genesis and brain distribution; such specific properties should be related to a specific functional role, which is still insufficiently understood.

\section{Sleep cycle or REM sleep generator?}

\author{
Serge Daan, Domien G. M. Beersma, and Derk Jan Dijk \\ Department of Zoology and Biological Psychiatry, Groningen University, \\ Haren, The Netherlands
}

Hobson, Lydic, and Baghdoyan present an exquisite review of the neurophysiological work inspired by the 1975 reciprocalinteraction model and at the same time reformulate this model as a basis for a further decade of sleep cycle research. In doing so, they provide the most explicit formulation of central nervous control of a behavioral state - REM sleep - to date. We feel that the predictive value of this model for the temporal organization of sleep will depend strongly (1) on its ability to quantitatively describe the alternation between three behavioral states NREM, REM, and waking - and (2) on the integration of more functional approaches to sleep states. We comment on these two issues.

(1) In its present formulation, the model does not generate quantitative predictions of the REM-NREM sleep cycle. The reason is that it emphasizes REM sleep generation and does not specify how the durations of NREM sleep and of wakefulness are differentially controlled. Both are associated with high activity of aminergic neurons. Dorsal raphe neuronal discharge patterns over a cycle were, for instance, quite similar despite a pronounced forward shift of NREM sleep at the expense of wakefulness in response to sleep deprivation (Lydic, McCarley \& Hobson 1984). This makes it presently impossible to derive from the model when REM sleep and when wakefulness will occur and hence to predict insomnia or hypersomnia. Predictions concerning total sleep as affected by stress and by drug facilitation of aminergic neurotransmission as presented by Hobson et al. actually concern total REM sleep. Several of the clinical phenomena listed in Table 4 concern total sleep and are thus not really accounted for by the model. For example, the reduction of hypersomnia in depression by tricyclic antidepressants (Table 4) cannot be explained simultaneously with the increase in total sleep time which is the usual clinical effect of tricyclics in endogenous depression (Kupfer, Spyker, Coble \& McPortland 1978). Much better support for the model comes from the virtually complete suppression of REM sleep by tricyclics. The simultaneous reduction of REM sleep and of wakefulness by these drugs in depression (Kupfer et al. 1978) highlights the need for further expansion of the model to account specifically for NREM sleep.

In addition to the distinction between NREM and waking, a quantitative elaboration of the model's interactions seems necessary to generate predictions. It is not clear whether the present model will generate a cyclic alteration between REM and NREM sleep. The existence of a periodic solution in the original McCarley-Hobson model depended on the choice of parameters for strength of reciprocal inhibition and excitation of the two postulated control centers (FTG and LC). These values were based on neuronal firing rates recorded from these nuclei. The broader concept of distributed neuronal control populations, although realistic, necessarily leaves these parameters unspecified: It is unclear whether they should be derived by fitting the model to DRN or LC firing rates, for example. With arbitrary parameters, the model can have both periodic and stable solutions.

(2) In its present formulation, the model does not specify how the organism's need for sleep will affect the control of sleep states by the putative neuronal generators. Hobson et al. do not address the vast literature on effects of total sleep deprivation and specific interferences with REM and NREM sleep. Changes in sleep electrophysiology with sleep satiation are wellknown for both NREM (Borbély \& Neuhaus 1979) and REM sleep (Aserinsky 1969). Such variations, supposedly reflecting variations in the intensity of both sleep states, will be of critical functional importance. Likewise, other functional variations in sleep cycle control - such as dependence on brain size (Zepelin \& Rechtschaffen 1974), age (Feinberg 1974), circadian phase (Zulley 1980), environmental temperature (Szymusiak \& Satinoff 1981), risk exposure (Lendrem 1984) - should eventually be accounted for by the cycle-generating mechanism. This is not to criticize Hobson et al. for their admirable adherence to neurophysiological evidence but to emphasize the need for integrated physiological and functional analysis. Behavioral manipulations in association with electrophysiological assays of neuronal activity (e.g., Lydic et al. 1984) should be as powerful in this respect as the pharmacological microinjections advocated by Hobson et al. A full understanding of the generating mechanism seems to require intricate knowledge about function. The function of the two alternating sleep states is conspicuously unknown. If neuronal repair requires sleep, functional disintegration of cortical information processing during NREM sleep and of hypothalamic regulatory activity during REM sleep suggest that the REM-NREM cycle primarily concerns the alternation between the sleep of the autonomic and the cerebrospinal nervous systems. Be that as it may, we should expect feedback circuits from the nervous system monitoring the progress of sleep to affect the putative pontine cycle generator. On 
the other hand, just as the analysis of mechanism requires a view of function, function will hardly be understood unless we are informed in detail about the generating mechanism. In this sense, Hobson and his colleagues have in their review made a major stride toward a functional concept of the sleep cycle.

\section{Reciprocal interactions in the brain stem, REM sleep, and the generation of generalized convulsions}

\section{Z. Elazar}

Department of Physiology and Pharmacology, Sackler Faculty of Medicine, Tel Aviv University, Ramat Aviv 69978, Israel

If found the new version of the reciprocal-interaction model to be in tune with factual and conceptual developments in the 10 years since its original conception. The following are my comments.

Selectivity. Today we are in possession of detailed analyses of firing patterns in populations of neurons considered to be executive for the main phenomena of REM sleep, cortical desynchronization, muscle atonia, PGO waves, and eye movements. As pointed out recently by McCarley and Ito (1985), despite this detailed knowledge, none of these executive populations of cells has been convincingly shown to be selective for REM sleep. Vertes (1984) has emphasized that none of the main REM sleep phenomena are unique to that state and that functions of REM sleep overlap with functions carried out in other states. If the REM sleep state is defined as the sum of its phenomenological signs, it is only the particular interaction, synchronization, and time course of the executive neuronal populations that is important. The concept of selectivity, conceived as exclusivity of firing in the REM state, is unnecessary for modeling the REM-sleep-generating mechanism. The search for such a selectivity should be abandoned, not only in connection with the FTG populations as Hobson, Lydic, and Baghdoyan have done, but also in connection with any neuronal population related to $\mathrm{REM}$.

The distributed nature of REM sleep mechanisms. I admire Hobson et al. for the scientific honesty and open-mindedness that brought them to this important shift from the original "centrist" model of REM generation. I have problems with accepting the extent of distribution proposed. The problem comes down to the need to define the term "REM-on cells." Since cells in many areas of the brain increase their firing rates during REM sleep it would be unhelpful to design a model of REM sleep generation that includes most of the brain. In Figure $l$ cells from the cerebral cortex to the cranial or spinal motoneurons are included in the REM mechanism. On the other hand, in the caption to Figure 2 REM-on cells are defined as those with REM firing rates twice as high as during SWS (slow wave sleep) or waking. As indicated in this caption, this restricts the populations of cells involved to the brain stem. This part of the brain was shown by a number of experimental methods to be both necessary and sufficient for the production of REM sleep. I therefore propose to restrict the distribution of REM-generating cell populations to those located in the brain stem. To make things clearer I propose to adopt the terms "BREM-on" and "BREM-off cells" (brain stem REM-on and off cells) when speaking about the generating mechanism of REM.

The BREM-off cell system. Hobson et al. emphasize the role of REM-off cells to a greater extent than they did in the original model. These cells appear to be the initiators of the chain of events leading to REM. Since the REM-off cells synchronize the activity of the executive neuronal populations, they could be critical for the generation of the state of REM sleep. Knowledge about the mechanisms inducing the reduction in firing in these cells could be important for testing the reciprocal-interaction model and for understanding REM generation. It will also be instructive to know the functional consequences of the dramatic decrease in the activity of BREM-off cells on their target cells in the spinal cord, hypothalamus, basal ganglia, and cortex. Research in this direction might shed light on the problem of the biological significance of REM sleep.

Future of the REM generation models. The future of REM modeling depends on research in two areas:

1. Neurochemistry. By using iontophoretic methods Greene and Carpenter (1985) found that both acetylcholine and serotonin, in addition to their respective excitatory and inhibitory effects on pontine neurons, also had inhibitory and excitatory or combined inhibitory-excitatory effects in equal proportion. It is probable that each of these neurotransmitters binds to several types of receptors with different locations on the neurons. In addition, GABA, glycine, aspartate, glutamate, and peptidic neurotransmitters influence cells in this region and may play a role in the generation of REM. The cholinergic-monoaminergic interaction proposed by the reciprocal-interaction model probably represents the interaction between the two main neurotransmitter pillars of the generating mechanism. When discovered, the role of other transmitters could complete or modify the present picture.

2. Circuit analysis. We now have a great deal of information concerning correlations between firing patterns and different phenomena of REM sleep. However, we need data about interactions at the basic neurophysiological level. In addition to the firing patterns, we need to study on what target cells the influence of a particular cell population is exerted, and the nature of this influence. The work of Steriade can serve as a model for this type of experiment (see Steriade et al. 1984). Other experiments along the same line have been performed by Fung, Boxer, Morales \& Chase (1982) and Sakai (see Sakai 1985a).

The reciprocal-interaction model and the REM-epilepsy problem. Recently we applied the assumptions of the reciprocalinteraction model to the issue of the relationship between REM sleep and epilepsy (Elazar \& Hobson 1985). This relationship is intriguing. On the one hand, there are similarities between the two phenomena: the similarity between the PGO spike and the epileptic interictal spike, the intensity of REM sleep phenomena in the postdeprivation period, the parallel between the enhancement of both REM phenomena and epileptic manifestations in the postdeprivation period, the unusually intense, internally triggered behavior during REM sleep without atonia in cats with lesions in the pons, and the dramatic increase in frequency of firing in pontine neurons in the transition from SWS to REM sleep. On the other hand, the increase in intensity of neural activity in the pons during REM sleep does not reach the level of epilepsy, and, moreover, the incidence of generalized convulsions during this state is low. We microinjected carbachol in the hippocampus, mesencephalon, and pons (Elazar 1985) and found that the minimal amount necessary to induce electrographic seizures or generalized convulsions was much higher in the pons than in the mesencephalon or hippocampus despite the fact that carbachol injected in the pons in lower amounts consistently induced signs of REM sleep. The resistance of the pons to epileptic seizures can be explained by a ceiling-setting mechanism probably consisting of inhibitory influences from monoaminergic neurons in the raphe and locus coeruleus nuclei. A decrease in the serotonin and noradrenaline content of the brain stem will "permit" the generation of epileptic seizures in the pons.

Another factor preventing generalized convulsions from occurring during REM sleep is the atonia. Different amounts of carbachol stimulation of the medial pons, a source of the reticulospinal tract, were shown to induce motor behaviors, REM atonia, or generalized convusions (Elazar \& Feldman 1986). We proposed a mechanism that would switch the activity of the mediopontine neurons from the mode of waking motor behavior 
to that of atonia or generalized convulsions. The monoaminergic neurons of DRN and LC were considered to be the critical factors for this switching mechanism. During waking, the active DRN-LC neurons exert their inhibitory effect on the mediopontine neurons, thus setting the optimal level for production of patterned movement. This inhibition is also exerted on peri-LC $a$ neurons, which weakens the atonia mechanism and permits the movement. During REM sleep the decrease in activity of the DRN-LC neurons will permit a higher excitability level in the mediopontine neurons, but will also desinhibit the peri-LC $a$ neurons and the atonia mechanism. During generalized convulsions, the strong epileptic drive will probably be exerted on both the mediopontine neurons and the DRN-LC neurons. The DRN-LC neurons would inhibit the atonia mechanism and the epileptic activity of the mediopontine neurons produces generalized convulsions. According to our model, waking motor behavior, REM sleep atonia, and generalized epileptic convulsions depend on the interaction between the pontine neurons involved in movement generation and the DRN-LC neurons. Testing the generation of generalized convulsions according to this model can contribute to the testing of the reciprocalinteraction model.

\section{Revising sleep cycle theory?}

\section{William Fishbein and Pnina F. Bright \\ Department of Psychology, The City College and Graduate School of the City University of New York, New York, N.Y. 10031}

The target article represents Hobson, Lydic, and Baghdoyan's attempt to revitalize Hobson and McCarley's 1975 reciprocalinteraction theory of REM sleep generation and regulation. Since 1975 there has been much more research, especially in the field of neurobiology of sleep, that has provided considerable evidence contradicting the theory and only a small amount of data that supports it. As a result, general acceptance of the theory has run into considerable difficulty.

The problem is that support for the theory continues to be based solely on correlative circumstantial evidence and not on data providing a critical test. The evidence, which includes studies of cell membership in distinct neurochemical groups, description of spike train characteristics, the neuroanatomical proximity of off cells to the locus coeruleus, the arrest of firing of cells following administration of pharmacological agents, shifts in cell membrane resting potentials, and studies of REM-off firing patterns, can, upon close scrutiny, be interpreted as reflections of neuroanatomical configurations and behaviors possibly related but also unrelated to REM sleep generation. The core of the problem is that the neuroanatomical projections of REM-off cells are not exclusively concentrated in REM-on cell nuclei, and no matter what arguments the authors bring to bear in order to back up their theory, they cannot change the neuroanatomical state of affairs that exists.

Despite all the criticisms Hobson et al. still remain wedded to their original idea. In order to deal with the criticisms or incorporate new findings into the theory, they introduce explanations that only complicate the theory further. For example, the demonstration of McCarley and Ito (McCarley \& Ito 1983; Ito \& McCarley 1984) that FTG cells depolarize before and throughout REM sleep provides some evidence that the FTG is a crucial component in the initiation and maintenance of REM sleep. To incorporate this new observation into the revised theory, Hobson et al. argue that the onset of REM sleep is achieved by the disinhibition of the FTG (the putative generator of REM sleep) produced by the attenuation of activity of the inhibitory amines from the locus coeruleus (LC) and dorsal raphe nucleus (DRN). The argument, however, introduces a neurobiological inconsistency. Removing the inhibitory influ- ences (i.e., disinhibition of the FTG) of norepinephrine (from the LC) and serotonin (from the DRN) would be expected to produce only a reduction in amplitude of hyperpolarizing potentials in the FTG and at best a return to resting-state membrane characteristics. Yet it is long-lasting depolarization of the FTG that McCarley and Ito observe during REM sleep. How do Hobson et al. explain this? According to the revised model there is no excitatory transmitter input into the FTG that could account for the depolarization effect. The alternative proposed by Hobson et al. is that the putative generator area(s) must have its own autoactivated pacemaker(s) that trigger REM sleep.

Hobson et al. believe that their theory can be extremely valuable in providing direction to the design of research strategies, some of which they quite directly propose in order to move the field away from the strategy of correlational observation to that of causal hypothesis testing. Certainly we can have no argument with this. The revision, however, appears to involve ever-increasing complexity.

The complexity does not bring us any closer to understanding the nature of sleep cycle generation but only complicates the solution by increasing the complexity of the explanation. The theory is now changed from a proposal of localized sleep centers to hypothesized interacting neuronal populations which are multiple, widely distributed, and interpenetrated. The bottom line, despite all the elaboration, is that there is not a shred of evidence that reciprocal interactions occur, and it may well be impossible ever to demonstrate with the research tools we presently have. Making the solution to these problems ever more complex by introducing qualifications and modification of the hypothesis suggests to us that Hobson et al. are distancing us even further from a solution.

There is certainly no shortage of alternatives to the reciprocalinteraction hypothesis, and these ideas may be even more parsimonious. What are needed are direct, concrete experimental approaches designed to alter the ultradian REM sleep clock by drug treatment or genetic manipulations and then determine the neurochemical site of action of the drug or the nature of the genetic manipulations. For instance, the ultradian rhythm of REM sleep might simply result from cyclic alterations in PRF (pontine reticular formation) cholinergic receptor sensitivity alone, without having to introduce the idea that inhibitory amine systems exert restraints on the cholinergic excitatory system. A first test of such a hypothesis that employed long-term microinjection techniques, like the ones described by Hobson et al., has already been reported (but not cited by the authors) and provides support for a more parsimonious, single-transmitter REM generator (Shiromani \& Fishbein 1980; 1986; Shiromani, Barnett \& Fishbein 1982). Future studies using measures of receptor sensitivity as detected by receptor binding assays could provide further support for such an idea.

Still another approach might focus on the influence of the suprachiasmatic nucleus (SCN) on pontine cell populations. For example, there have been some recent suggestions that this diencephalic circadian oscillator, which has only recently been shown to be a sexually dimorphic (genetic) structure (Swabb, Fliers \& Partiman 1985), might regulate the ultradian brain stem oscillator(s). This idea emerges from two separate findings: (1) that the regulation of sleep cycles is sexually dimorphic (Branchey, Branchey \& Nadler 1973; Yamaoka 1980; Wever 1984; Fishbein \& Bright 1986) and (2) that vasoactive intestinal peptide (VIP), which is densely concentrated in the SCN (Card, Breecha, Karten \& Moore 1981), appears to influence the brain stem trigger mechanism as measured by its effects on REM sleep, which is augmentation (Riou, Cespuglio \& Jouvet 1982; Drucker-Colin, Bernal-Pedraza, Fernandez-Cancino \& Oksenberg 1984). A recent examination of VIP distribution in the brain stem shows that the highest concentration of this peptide is confined to structures in the region of the fourth ventricle (Eiden, Nilaver \& Palkovitz 1982), the same brain stem areas that are involved in REM-generating functions. Furthermore, 
VIP has been demonstrated to occur mostly in cholinergic neurons (Lundberg, Hokfelt, Schultzberg, Uvnas-Wallenstein, Kohler \& Said 1979), suggesting that the ultradian oscillator might be influenced by VIP-mediated cholinergic action.

What emerges despite Hobson et al.'s impressive attempt to explain the generation of sleep cycles is the conclusion that this is a complex problem which requires a fresh examination rather than a reformulation of an old theory.

\section{Sleep-cycle generation: Turning on, turning off, and tuning out}

\author{
Stephen L. Foote \\ Department of Psychiatry, University of California, La Jolla, Calif. 92093
}

Hobson et al.'s proposed model is grounded in numerous observations and is heuristically rich. It makes a large number of interesting, specific predictions that can be tested with presently available technology. This commentary suggests further elaborations of three specific areas, two conceptual and one technical.

What turns on REM-on cells? The absence of aminergic tone during REM sleep may indeed contribute to the activation of REM-on cells during this state. Independent evidence has led to the suggestion that during the waking state norepinephrine release facilitates vigilance processes, that is, maximal attentiveness to external stimuli and minimal control of various brain regions by autoactivation, recurrent networks, recruitment, or "intrinsic" processes (reviewed in Foote, Bloom \& Aston-Jones 1983). The absence of norepinephrine release during REM sleep would presumably have converse results. Although we have put forward this proposal mainly in terms of the projections of the locus coeruleus and raphe nucleus to the neocortex, it is completely compatible with the description in the target article of aminergic effects on REM-on neurons. However, the term "disinhibition" to describe the effects of reduced amine release may be misleading. Norepinephrine seems to alter gross activity levels by reducing spontaneous activity, but its primary effect is to alter the type of activity rather than the amount of activity. One could suggest that in the visual system, for example, aminergic afferents participate in determining the difference between visual perception and hallucinosis (or dreaming). The crucial difference is not that une involves more neural activity than the other; it is that different modes of processing are engaged. One is intrinsically generated; the other is highly dependent on external stimuli.

Thus, it is reasonable to suggest that the inactivation of the massive efferent projections of REM-off neurons during REM sleep, combined with a moderate level of excitatory afferent input, could produce REM-on activity.

What turns off REM-off cells? It is the position of the target article that there must be a network of cholinergic neurons, no one subset of which is necessary and sufficient for REM sleep generation, which act as an ensemble to generate REM sleep. However, it seems likely to this commentator that some of these REM-on subsets are more important than others. The essential subsets are presumably those which participate most directly in producing the lack of activity exhibited by REM-off neurons during REM sleep. The crucial missing link in the diagrammatic presentation of the reciprocal-interaction model is the element responsible for the suppression of activity in REM-off populations during REM sleep. How is the profound inhibition of REM-off neurons during REM sleep maintained? How is the postulated excitatory cholinergic input to REM-off neurons, presumably very active during REM sleep, overridden? The previously proposed criteria of selectivity and tonic latency of activity can suggest candidates for this crucial subset of REM-on neurons, but combined anatomic-physiologic criteria will be crucial to the demonstration that some particular set of neurons fulfills this function. Such neurons are certainly a necessary (and possibly sufficient) component of the REM-sleep-generating ensemble. The target article notes that FTG stimulation has state-dependent excitatory effects on DR, which become minimal or nonexistent during REM sleep. Thus, whereas the proposed excitatory input from REM-on to REM-off cells may be minimal during REM sleep, it cannot explain the profound suppression of REM-off cells during this state (Foote, AstonJones \& Bloom 1980; Aston-Jones \& Bloom 1981). Thus, the proposed model highlights the importance of determining what are the afferents to REM-off neurons and which of them might be responsible for their REM-off behavior.

Hobson et al. propose that an extremely low ratio of aminergic/cholinergic activity may be the defining characteristic of REM sleep. This is an interesting, appealing, testable proposal. It would seem, then, that the crucial question is, "What are the anatomical substrates and physiological events that precipitate and maintain a dissociation (inverse relationship) between the activity of cholinergic and aminergic neurons during REM sleep?" A slight extension of the proposed model would indicate, as suggested above, that these "dissociative/inverse" events are the necessary and sufficient neural events of REM sleep. Many of the observed signs of REM sleep could more usefully be viewed as consequences of this dissociation rather than as intrinsic to REM sleep. Clearly, some of the consequences might be the function of REM sleep, but they might well not be its essence. For example, one function of REM sleep might be to dramatically reduce the ratio of aminergic versus cholinergic transmitter release onto cortical neurons.

What is different about microinjection experiments? The technical issue to be addressed is the usefulness of microinjection manipulation of certain neuronal populations. The stated goal of the target article "is to move beyond the debate about the significance of correlations (between cellular discharge patterns and sleep states) and to stimulate a new era of causal hypothesis testing by reformulating the reciprocal-interaction hypothesis in terms more amenable to unambiguous empirical evaluation.:" Whereas it may be true that the types of manipulative experiments proposed here permit relatively more direct tests of causality than do correlational studies, and that these manipulative studies are more interpretable than lesion studies, at least two problems remain: (1) there are still artifacts associated with pharmacological manipulation (i.e., pharmacological manipulation cannot exactly mimic physiological conditions), and (2) in a network with distributed functions, manipulations of one node in the network may not produce (or delete) the physiological signs that normally result from the activation (inhibition) of that node. Although pharmacological activation may more closely mimic physiological conditions in this type of network than does an irreversible lesion, the difference is one of degree and not of kind. It may well be true, as suggested by Hobson et al., that not even recording and lesion data in combination are sufficient to demonstrate causality, and that the pharmacological data would be useful in enhancing our confidence about statements of causality.

One reason that recording and lesion data are not conclusive is that the simple linear, hierarchical, unidirectional model of causality conjured up by the statement that nucleus $\mathrm{X}$ causes behavior $\mathrm{Y}$ is probably not operating in behavioral-state control. It would probably be much more accurate to say that nucleus $\mathrm{X}$ participates in the normal, full elaboration of behavior $Y$. The target article directly and clearly addresses this issue at the conceptual level, for example, in the discussion of the network organization of REM-on cells. The problem is that it is not clear how the ways in which localized pharmacological manipulation and the lesion method deal with this type of causality differ qualitatively.

Perhaps the most important purpose served by the target article is its convincing demonstration that to acknowledge that 
brain functions are dependent upon distributed structures, and to acknowledge the resulting lack of conclusiveness of lesion and correlative physiological data, does not leave neuroscientists impotent to deal with important functional questions, and even questions of causality, using presently available technology.

\section{Sleep cycle generation: Testing the new hypotheses}

\section{Robert Freedman}

Departments of Psychiatry and Pharmacology, University of Colorado Health Sciences Center; Denver V.A. Medical Center, Denver, Colo. 80262

What are the key concepts in sleep cycle generation that have evolved as a result to Dr. Hobson's group's data and reflections? Could one simply say that they have not yet looked at the "right" population of trigger neurons for REM sleep? Or have they indeed conceived a new way of looking at the neuronal control of behavioral change that leads to a better interpretation of the wealth of data on changes in neuronal discharge during sleep cycles?

Hobson et al. present a convincing argument that sleep cycles are not easily explained by the concept of ascending levels of control, with specific centers being ultimately responsible like homunculi for switching behavioral states on or off. They highlight two findings of sleep research that contradict the existence such mechanisms: (1) the presence of simultaneous changes in several nuclei with quite different chemical constituents and (2) the "interpenetration" in anatomical centers of cells with opposing valences of change in discharge rate. The second concept is unique to Hobson's group and is worthy of the emphasis placed on it. What less creative experiments might regard as a "problem" in the data Hobson et al. have turned into a strength. Interpenetration is a unique enough idea, however, so that it requires a review of the experimental data supporting it. Are localization techniques adequate to assess interpenetration? Are cells within locus coeruleus, raphe, and reticular formation differentiated or homogeneous by chemical, physiological, and anatomical analysis? Do any differences account for the interpenetration Hobson et al. postulate?

Hobson et al.'s model might account for a phenomenon in sleep cycle generation which they did not discuss but which might form a useful test of their ideas. All sleep researchers know that short or abortive REM cycles can occur, particularly early in the night. These failures in sleep cycle generation may illuminate problems in the coordination between centers, which is an important aspect of Hobson et al.'s hypothesis of cycle generation. They and other sleep researchers probably already possess many data on discharge changes in these cycles, perhaps even from the same cells that have been recorded during more prolonged state changes. Analysis of the action of various cell centers during these "failures" might provide the important control data needed for the hypothesis. How do cells change firing if the attempt to change state is aborted? Is some aspect of linkage between centers critical, or, as in a Sherringtonian model, is only one center crucial?

\section{Modeling sleep: We need all the perspectives we can get!}

\section{Ramon Greenberg}

Haward Medical School: Massachusetts Mental Health Center, Boston, Mass. 02115

It is rare that scientists write a paper that so clearly incorporates new information, much of it provided by other investigators, in order to suggest changes in their own scientific model. Hobson and his colleagues have done just that and are to be commended for it. In the spirit of this openness, and in the hope of continuing and furthering this evolution, I would like to discuss their target article and the evolving model from the point of view of isomorphism, a concept that Hobson and McCarley made much of in their original formulations (1977). Unfortunately, however, they saw isomorphism as going in only one direction. They used the notion of the importance of consistency between neurophysiologic and psychologic realms to raise some important questions about Freud's early theories of dreaming. Their criticisms of those early ideas of Freud were, for the most part, well taken, but, by failing to consider later psychoanalytic thinking about dreams and evidence for the role of higher brain centers in REM sleep, they wound up with the pons in too central a position. The present work corrects that narrow focus. I would like to show how this could have been anticipated by taking isomorphism more seriously. I also suggest that a continuing attention to other perspectives can help in the development of this evolving model.

To begin with, although the criticisms of Freud's drive theory in relation to dreaming have some merit, there is abundant clinical and laboratory evidence to confirm the idea that dreams are not just random perceptual images but have a coherent meaningfulness in their manifest content. This meaningfulness of dreams can be related to the REM process, and this is the point where Hobson and McCarley lost the opportunity to consider the interaction of multiple and anatomically distributed sets of neurons in their reciprocal-interaction model. This can best be explained by considering the evidence for the role of dreaming and REM sleep in adaptation. Psychoanalytic writers following Freud (Meader 1916, French \& Fromm 1964, Bonime 1962, Palumbo 1978, and Greenberg \& Pearlman 1978 , to name a few) have shown how dreams, including the manifest content, can be understood in terms of the dreamer's efforts to deal with ongoing, central emotional problems.

Evidence that the manifest dream is related to direction of eye movement (Roffwarg et al. 1962) and middle ear action potentials (Roffwarg et al. 1975) suggests that the dream is not just an epiphenomenon or associations by the cortex to random firing from the pons, but rather that the REM process and dreaming are intimately connected. Thus a role for the cortex should have been considered earlier. Hobson et al. do note some of the evidence that damage to the cortex affects eye movements during the REM period, but an isomorphic approach would have made this more apparent. Going further, we should note the results of a variety of learning and adaptive tasks in relation to REM sleep. REM deprivation has led to impairment of task mastery in both animals (see Pearlman 1981) and humans (see Greenberg 1981), and, of even more importance to this discussion, the challenge of such tasks has been shown to change the timing and quantity of REM sleep. If one takes isomorphism seriously then consistency requires considering the role of neurons in higher centers in the timing of the REM process. Hobson et al. imply this when they note that anxiety may affect the timing of REM activity.

The importance of noting these findings is that research into REM sleep has the potential to be even richer in elucidating the variety of interactions than the present expansion of the model suggests. What must be kept in focus is that experience affects the action of the neuronal networks described in the target article and the action of the networks affects the behavior of the animal or human that has REM sleep available. If we take this seriously, we have the possibility of performing new and exciting experiments. For example, will selectively blocking different parts of the networks by microinjection lead to more precision in our REM deprivation studies and help us learn more about the information networks in the brain? Can activation of certain parts of the networks enhance the adaptive effects of REM sleep? Can microrecording help us define the areas of the brain which are activated by REM-sensitive experiences? 
The elegant studies described in the target article have demonstrated the complexity of the REM process. I have tried to show the further extent of the interactions that must be considered, and I hope that this will contribute to the development of a consistent and coherent model of "reciprocal interaction" in the fullest sense of the term.

\section{Wet physiology of REM sleep generation}

\author{
W. Haefely \\ Pharmaceutical Research Department, F. Holfmann-La Roche \& Cie. Ltd., \\ CH-4002 Basel, Switzerland
}

In Hobson et al.'s proposed reciprocal-interaction model of REM sleep generation the global inhibitory action of noradrenergic (NA) and serotoninergic (5-HT) neuron populations occupies a central position. Our own studies have dealt almost exclusively with this component of the model. We induced state-independent pontogeniculooccipital (PGO) waves in the unanesthetized curarised cat by either preferentially depleting $\mathrm{NA}$ with the benzoquinolizine Ro 04-1284 $\left(\mathrm{PGO}_{1284}\right)$ or by rather selectively inhibiting the biosynthesis of 5-HT with pchlorophenylalanine $\left(\mathrm{PCO}_{\mathrm{PCPA}}\right)$. Our systematic investigation of hundreds of agents for their modulatory action on PGO waves (Monachon et al. 1972; Jalfre et al. 1974; Haefely et al. 1975; 1976; Ruch-Monachon et al. 1976) produced a wealth of data which could be explained easily and parsimoniously by assuming that activating receptors for NA and 5-HT depresses the frequency and duration of PGO wave bursts, whereas reducing the activation of the receptors by any means (blockade of receptors, decrease of NA and/or 5-HT release) initiates or facilitates PGO activity. It was also evident that the 5 - $\mathrm{HT}$ system is a more powerful suppressor of PGO activity than the NA system.

It was of obvious interest to use the available pharmacological armamentarium for a study not only of the PGO component but also of the sleep cycle in freely moving cats. An excellent correlation was found between the effect of drugs on $\mathrm{PGO}_{1284}$ and $\mathrm{PGO}_{\mathrm{PCPA}}$ in the curarised cat and their effect on REM sleep in telemetrically recorded cats (Polc et al. 1979; Scherschlicht et al. 1982). All drugs with establishcd antidepressant activity selectively reduced the amount of REM sleep. The most potent drugs were those with a powerful inhibitory action on the neuronal reuptake of 5-HT. The more specific inhibitors of NA reuptake required higher doses for the selective suppression of REM sleep. As mentioned in the target article, these findings raise several questions concerning REM sleep and mood disorders.

The consistent finding of a reduced spontaneous firing rate of neurons in the locus coeruleus (LC) and dorsal raphe nucleus (DRN) during the transition from NREM to REM sleep (REMoff) provides the neurophysiological basis for our crude pharmacological experiments. One would then assume that the drugs which reduce REM sleep selectively (mainly antidepressants) do so by preventing the synaptic concentration of 5-HT and/or NA to decrease below a critical value that can inhibit REM generation, in spite of a marked reduction in the firing rate of monoaminergic neurons. A test of this assumption would be to record $L C$ and DRN neuronal activity after administrating an antidepressant to see whether it still shows the usual intrasleep slowing in the absence of actual REM sleep phases. The suppressant effect of NA and 5-HT neurons on REM sleep generator neurons implies that NA and 5-HT act as inhibitory neurotransmitters. Now, a simple inhibitory function for these monoamines appears to be rather unlikely, as recent studies have revealed very complex synaptic action of NA and 5-HT.
Besides an inhibitory component, these amines also possess some activating properties, such as reducing the frequency adaptation of action potentials to direct depolarizing pulses. Their overall synaptic effect is modulatory rather than simply inhibitory or excitatory, resulting in an increased signal-to-noise ratio of the synaptic input. The types of NA and 5-HT receptors mediating the assumed inhibitory influence of the monoamines on REM generator neurons have not been clearly identified, though our previous studies suggest that NA may act through $\alpha$ adrenoceptors rather than through $\beta$-adrenoceptors.

The anatomical and neurochemical identity of REM-on cells occupies a longer part of the target article. The possibility of inducing complete REM sleep or isolated REM sleep components by local microinjections of cholinomimetic agents and anticholinesterases into the brain stem is a powerful approach to the study of REM sleep generation. Hobson et al. have characterized the cholinergic induction of REM sleep as muscarinic. The question whether cholinergic synapses are involved in the modulation of REM-on cells or whether REM generator neurons are cholinergic themselves appears to me to be quite open. So far the types of neurons directly affected by the injected cholinomimetics have not been identified. From our previous studies we concluded that muscarinic cholinoceptors were more probably involved in the regulation than in the generation of REM sleep phenomena. In our hands, atropine had no significant effect on $\mathrm{PGO}_{1284}$ (owing predominantly to an elimination of the noradrenergic "brake"), but dose-dependently decreased the density of $\mathrm{PGO}_{\mathrm{PCPA}}$ (owing to a rather selective removal of the 5-HT "brake"). I still feel that these findings indicate the existence of an excitatory muscarinic step within the neuronal input to the LC (Ruch-Monachon et al. 1976).

A very striking finding in our studies of PGO pharmacology concerned nicotine and the antinicotinic agent mecamylamine. These were the only drugs that reduced the amplitude of single PGO waves. In contrast, they failed to affect the timing of PGO wave bursting. These findings are compatible with the assumed cholinergic nature of pacemaker or generator cells, and they suggest that cholinergic synapses operating through nicotinic cholinoceptors are physiologically involved in the recruiting of generator cells by modulator cells or by generator cells themselves. Unfortunately, we have not yet studied the existence of the nicotinic mechanism in the generation of complete REM sleep. It would be important to extend the local microinjection studies using pure nicotinic cholinomimetics (nicotine, dimethyl-phenylpiperazinium) and pure antinicotinic drugs (such as mecamylamine).

Most synaptic effects of acetylcholine ( $\mathrm{ACh}$ ) in the brain are believed to be mediated by muscarinic cholinoceptors, although some effects of $\mathrm{ACh}$ were blocked by antinicotinic agents and specific binding sites for nicotine were demonstrated. It is quite conceivable that exogenous nicotine excites cholinergic neurons, which then release $\mathrm{ACh}$ into muscarinic cholinoceptors. Noncholinergic neurons may also possess nicotinic cholinoceptors. The use of antinicotinic agents in the identification of nicotinic cholinergic transmission should therefore be pursued in preference to the use of nicotinic stimulants, which may activate purely pharmacological receptors (not activated by endogenous $\mathrm{ACh}$ ).

It is also highly desirable to examine more precisely the diffusion of injected cholinergic agents at different times after local injection (e.g., by autoradiographic visualization of radiolabelled compounds). Such studies could, for example, explain the surprisingly delayed REM increase after the microinjection of cholinergic drugs.

A general note of caution concerns the extremely high drug concentrations used in microinjection experiments. Drug concentrations are extremely high in the center of the tissue area exposed to agents (Heule et al. 1983), and many drugs are likely to produce very different (even opposite) effects on neurons located at various distances from the injection point. 


\section{The elusive sleep cycle generator}

\section{Henn}

Department of Neurology, University Hospital, CH-8091 Zürich, Switzeriand

The year 1975 gave us the sleep cycle theory based on the interaction of two well-localized and functionally well-defined neuron populations. The year 1985 gives us the theory in a more refined form: Neurons are distributed in a network but are still composed of two well-defined antagonistic sets. I would like to predict that the theory will be further extended. The concept will be introduced that there are not just two sets of neurons but several subsystems displaying cyclic activity of different periodicity. The subsystems show some degree of interdependence and are normally synchronized to generate the sleep-waking pattern. Then the experimental difficulty will be (1) to measure the activity of the relevant neurons if they defy localization in the classical sense (as being part of a distributed system) and (2) to link their activity in a causal way to the sleep cycle (if their own activity represents just one of several subsystems and need not necessarily have the same pattern and length as the sleepwaking cycle).

The original sleep cycle theory was not much more than the formal description of the phenomenon. That neurons can be found in the brain stem whose activity can be related to a behavioral state does not "explain" much, just as neuronal activity in the motor cortex preceding motor acts is not an "explanation" of motor behavior, at least not in the year 1985. It just demonstrates that specific EEG changes or other phenomena have some as yet undefined causal relationship to sleep. One puzzling phenomenon is the profound effect of the various sleep cycles on the motor system. Beyond the mere description it seems to be unclear whether this is entirely an epiphenomenon or whether central generators of motor programs play some interactive role. Is this the right way to go then? Yes, but one would have to go much further.

I especially value the concept of behavioral state and its control. For too long have neurophysiologists recorded activity in various areas of the brain without considering the state of the animal - often some kind of coma. Even if so-called alert preparations are used with the various encéphale isole operations, the ensuing brain edema, altered blood circulation, and shock change the state so that it is far from normal. That Hobson et al. precisely describe "states" and define how sets of neurons alter their activity while the "state" changes is an important contribution; I only wish they had gone further. As sleep researchers it is their privilege to concentrate on the various substates of sleep. But one would wish to know how these same sets of neurons are active during waking. To determine the causal role of these cells would require investigating them during all different stages of sleep and waking, and also while the animal executes a wide variety of motor programs. This will be a formidable task, but it is technically feasible, and I do not see any shortcut around it. It seems that sleep researchers mostly define waking as nonsleep. This is just not sufficient; indeed, it is very misleading if animals are partially restrained, are in a sensorily deprived surround, and cannot interact with their environment.

An important development is the experiments aimed at locally interfering with the system. As in other areas of neurophysiological research, local injections of toxin to selectively kill cells, or transmitter agonists or antagonists to temporally block transmission, are promising new tools. Various experiments have shown that rather large chemical lesions in the pontine tegmentum can lead to focal neurological deficits (investigated in the most detail in the oculomotor system) but have little effect on sleep behavior.

Some further aspects have not been considered. For example, there are other well-known cyclic phenomena such as serum cortisol level, core temperature, or other circadian patterns. To what extent can these phenomena be dissociated in nonharmonic patterns from each other? In humans, consideration of pathological states such as narcolepsy or the changed frequency of REM sleep in depressive patients could suggest further insights and may have to be considered in the formulation of a theory.

Finally, one might ask whether measurements of spiking activity are the best parameters to characterize such relatively slow cycling events. How can local blood flow, glucose utilization, or other indicators of local activity be related to sleepwaking patterns?

In conclusion: The experiments Hobson et al. have performed have taught us a lot, but they have not given an answer I would consider valid or sufficient. Regarding their theories, one must point out that they are simplistic and that there exists overwhelming complexity which at the current stage defies even the formulation of a testable model. Whether or not one to takes Hobson's side I consider more a matter of temperament than a scientific issue. Hobson and his coworkers have gone ahead and formulated a dualistic hypothesis; it takes so much less effort to criticize it, even if many of us would argue that we are still in the prescientific stage of hunting and gathering facts. To take a look back: Science has always produced two types: the simplifiers and those who see problems in every theory. They are not confined to particular areas of the globe - they are distributed. From its individual stand point each often considers the other side to be wrong - but they need each other to define their own stand point, and in a reciprocal fashion the waxing and waning of their influence determines the current state of science.

\section{Doubt and certainty in the neurophysiology of state}

\section{Steven J. Henriksen}

Division of Preclinical Neuroscience and Endocrinology, Research Institute of Scripps Clinic, La Jolla, Calif. 92037

The biological significance of the cyclic alternation of mammalian states of arousal remains one of biology's fundamental enigmas. There does not yet exist a satisfactory explanation for the biological need for such state changes, nor does there exist sufficient physiological evidence to explain the underlying processes leading to the transitions between these states. This is remarkable, given the fact that for what appear to be more complex forms of mammalian behavior - e.g., eating, drinking, sexual activity, speech, and memory processing - there are well-developed, working anatomical and neurophysiological models. Indeed, without a similar functional and intuitive perspective, sleep researchers have been left to try to gather their data in the dark, so to speak, hoping that the biological basis of arousal states will follow from a more complete description of the underlying events correlated with changes of state. Unfortunately, this approach results in the analysis of many interesting but functionally unimportant epiphenomena of the various behavioral states (e.g., REM-associated nocturnal penile tumescence). However, short of a dramatic change in our understanding of why these states exist, it remains for physiologists to continue such data-gathering efforts, and to incorporate meaningful insights derived from such experimentation into revised models. The target article by Drs. Hobson, Lydic, and Baghdoyan represents such an attempt. In it Hobson et al. reassess proposed mechanisms underlying sleep cycle generation and attempt to reconcile constructively what they now admit to be substantive criticisms of their own existing paradigm, the "reciprocal-interaction" model.

In its simplest and earliest form the reciprocal-interaction hypothesis proposed that there was a formal, even mathematical, relationship between certain very circumscribed neuronal groups in the pontine tegmentum and reticular formation: 
Their reciprocal reinnervation elaborated REM sleep cyclicity. The experimental evidence for this mechanism came primarily from the relative reciprocal selectivity of two populations of brain stem neurons for REM sleep. These original neurophysiological observations, however correlative in nature, served to direct the attention of many sleep physiologists away from the strict neurochemical explanations of state to the rigorous analysis of single neuronal activity and behavior. This impetus may remain as the most lasting achievement of this hypothesis. The original construct provided numerous predictions, most of which have, in one form or another, been rigorously tested over the last decade. In my opinion, the outcome of these studies has clearly failed to support most of the basic tenets of the original hypothesis. Hobson et al. have thoughtfully attempted to deal with the major substantive criticisms of their hypothesis. In sum, it appears to me that the current reformulation of the reciprocal-interaction hypothesis is, in fact, a significant rejection of the major tenets of the original. Nevertheless, the authors curiously choose to retain the original descriptive name. At what point does such a major conceptual change deserve a new name? In my opinion, the present target article represents such a point.

As Hobson et al. eloquently recount, current evidence points to "REM-on" cells as being considerably more distributed than originally conceived, and with no convincing specific interaction with the enlarged class of aminergic "REM-off" neurons. In addition, the nature of state selectivity for the critically important neurons remains substantially unsupported. Although the authors argue that these facts taken by themselves are not sufficient evidence for rejecting possible reciprocal interactions of some class of state-related cell assemblies, it certainly takes a stretch of the imagination to believe that the current evidence supports, even remotely, the original conceptualization. It appears that the current framework encompasses larger cell assemblies with far more complicated interactions, where a mathematical model of interaction may represent, at best, a special case. The present rendition of the model comes far closer to more inclusive models of others (see McGinty 1985), in which widespread equilibrium processes, utilizing multioscillator networks, are hypothesized to underlie state changes. However, Hobson et al. have provided a significant opportunity for sleep physiologists to review the evidence objectively and to determine for themselves whether the existing model successfully explains the wealth of pertinent data. Many of the invited commentators will have covered specific points of interpretation of existing data and will eloquently argue their cases pro or con. What is growing clearer to me is that a simple model of state regulation (however elegantly conceived) will no longer suffice to explain the steadily increasing complexity of observed neuronal interactions subserving state regulation. Several of the unexpected complexities of state have been uncovered by the authors themselves (e.g., the anatomical specificity for cholinergic induction of independent components of REM sleep). Hobson et al. are to be praised for their consistent efforts to refine their hypothesis ever more clearly; they only can be faulted only for failing to recognize its major evolution. One may be sure that this group will remain a major conceptual force in the study of state regulation.

Nevertheless, as a prerequisite, newer models of mammalian state generation will be needed to integrate emerging neurobiological principles. These include (1) the role of the growing list of ubiquitous neuropeptides in state regulation; (2) the impact of the widespread coexistence of neuroactive substances, and their demonstrated impulse- (frequency-)dependent release; (3) the clarification of the synaptic sign of specific neuroregulators given the multiplicity of receptor-coupled ion channels and their voltage-dependent characteristics; (4) the role of local control of transmitter release in postsynaptic neuronal excitability, irrespective of presynaptic discharge; and (5) the possible role of ultradian changes in ligand efficacy due to changes in receptor affinity, or coupling. Several of these events will most certainly be involved in, and some may be at the heart of, the neuronal mechanisms underlying the elaboration of the currently defined arousal states.

\section{The biological purpose of sleep may make multiple distributed reciprocal systems meaningful}

\section{Herbert H. Jasper}

Centre de Recherche en Sciences Neurologiques, Faculté de Médecino, Université de Montréal, Montreal, Quebec H3C 3J7, Canada

The evolution of concepts of functional localization in the brain from centers and local cortical areas to more widely distributed cell assemblies, distinguished more by their neurochemical identity than by their morphological topography, has been applied by Hobson et al. to the development of a reciprocalinteraction model of the control of sleep cycles. In this model the interaction of cholinergic and aminergic neuronal systems is emphasized, with possible supplementary roles played by amino acids (GABA) and certain peptides. Particular attention is given to the control of states of REM sleep linked to a critical balance between serotonergic, noradrenergic, and cholinergic neuronal networks originating in the brain stem and diencephalon. However, not enough attention has been given, in my opinion, to the control of states of wakefulness, arousal, and attention and to states of slow wave sleep.

For example, there is abundant evidence for the involvement of cholinergic mechanisms in arousal and wakefulness, and probably also in mechanisms of memory. Cholinergic mechanisms also seem to be involved in REM sleep, a quite different state of behavioral reactivity in which memory mechanisms are impaired or absent and movements are arrested with inhibition of spinal reflexes. The involvement of widely distributed cholinergic mechanisms intermingled with aminergic systems in states of REM sleep as well as in wakefulness shows that it is the functional anatomic organization as well as chemical reciprocity that determines states of sleep and waking.

It is certainly a significant step forward to demonstrate that neuronal systems of different chemical identity involved in controlling cyclic changes in states of sleep are widely distributed, but this still leaves us with a complex of reciprocally interacting systems associated with both sleep and waking with little understanding of just how and why these most important differences in general states of reactivity of the brain are brought about. What is the basic neurobiology of sleep and waking, and of the difference between REM and slow-wave sleep?

Hobson et al. are well aware of these problems and of the danger of inferring causality from observations of the firing of single cells during different states of sleep and waking. They also suggest a means whereby the "sleep state oscillator" may be controlled by circadian rhythms of hypothalamic origin. It may be that all of these various factors interacting in regulating states of sleep and waking will eventually be better understood as different parts of the overall picture of biologically purposeful states of rest or reactivity of the organisms.

\section{The need for a new model of sleep cycle generation}

\section{Barbara E. Jones}

Neuroanatomy Laboratory, Montreal Neurological Institute, Department of Neurology and Neurosurgery, McGill University, Montreal, Quẹbec, H3A $2 B 4$ Canada

Fifteen years ago, McCarley and Hobson (1971) brought forth evidence from single-unit recording that neurons in the pontine 
gigantocellular tegmental field (FTG) discharged selectively during paradoxical sleep (PS). This "discharge selectivity" was put forward as a basic criterion for an active role of FTG neurons in the generation of paradoxical sleep. Subsequently Hobson, McCarley, and Wyzinski (1975) showed that locus coeruleus (LC) neurons decreased firing during paradoxical sleep, and they hypothesized that this population of cells might inhibit those neurons generating PS. Based on the inverse activity curves of the two cell groups, they postulated that a "reciprocal interaction" between excitatory (FTG) PS generator and inhibitory (noradrenaline, NA LC) neurons determined the alternation of sleep cycle states (McCarley \& Hobson 1975b).

These original hypotheses and the emergent model have failed to stand up to empirical testing over the last 10 years. First, the activity of the pontine FTG neurons was not found to be selective to PS (Siegel \& McGinty 1977) but to be correlated with specific movements during waking in the unrestrained cat. Furthermore, destruction of the FTG neurons by kainic acid (Sastre, Sakai \& Jouvet 1981) or delimited thermolytic lesions (Friedman \& Jones 1984) failed to eliminate or significantly disrupt the state. Second, although other researchers have also found that NA LC neurons ceased firing during PS, no one has produced evidence that these neurons exert an inhibitory influence on the pontine FTG neurons. It has also been found that the NA LC neurons do not play a critical role in the cyclic appearance of paradoxical sleep (Jones, Harper \& Halaris 1977). Third, no direct interaction between the pontine FTG cells and the NA LC cells has been demonstrated. Neuroanatomical studies have not revealed an innervation of the LC by the FTG neurons but have instead shown a dense projection from the FTG into the paramedial brain stem reticular formation and the ventromedial ventral horn, indicative of involvement in motor function (Jones 1980). Reciprocally, histofluorescent studies have not revealed a very dense noradrenergic innervation of the pontine FTG but have instead indicated a minimal number of varicosities in this region as compared to other dorsolateral regions of the brain stem reticular formation (Jones \& Friedman 1983).

Now, some 10 years later, Hobson, Lydic, and Baghdoyan have reformulated their model in an attempt to accommodate these negative findings and incorporate new data and evolving concepts in the field. They have expanded the category of PS "generator" neurons to include "an anatomically distributed population" of excitatory, "cholinergic/cholinoceptive" PS-on cells in the brain stem (thus including cells distributed through the medullary, pontine, and mesencephalic reticular formation), and they have described these neurons as interacting with motor control systems during wakefulness as well as during PS. Second, in the category of "modulatory" cells, which would be inhibitory to PS sleep generator neurons, they have included monoaminergic PS-off cells located in the locus coeruleus, the raphe nuclei, and the "peribrachial region." Third, they maintain that a "reciprocal interaction" occurs between excitatory, "cholinergic/cholinoceptive" PS-on cells and inhibitory, monoaminergic PS-off cells but that the distribution of these two cell groups is so widespread, and the "interpenetration" of the PSon and PS-off cells so great, that localization of generator and modulator populations and their interaction in the brain may be impossible.

In this revision of the original reciprocal-interaction model, Hobson and his colleagues have expanded the membership of the generator and modulator populations, but they have also totally changed the basic assumptions of the original model. They have dispensed with the fundamental criterion for PS generator neurons, that is, "discharge selectivity" during PS, and allowed that these cells be active during wakefulness as well. In the absence of this principle, the cyclic generation of PS can no longer be explained by the activity of PS-on cells due to cessation of firing of the inhibitory PS-off cells or by the re- ciprocal interaction of the two populations of neurons. Without this criterion, the category of PS generator neurons, now characterized simply as PS-on cells, has become so large as to include the entire brain stem reticular formation. Furthermore, the generalization that reticular neurons which make up this large category of PS-on cells are "cholinergic/cholinoceptive" and excitatory is not supported by histochemical and pharmacological data. In contrast to early evidence utilizing acetylcholinesterase histochemistry and choline acetyltransferase (ChAT) immunohistochemistry with polyclonal antibodies (Kimura, McGeer, Peng \& McGeer 1981), recent ChAT immunohistochemical results with monoclonal antibodies indicate that a relatively small number of cells in the brain stem reticular formation contain the synthetic enzyme for acetylcholine and are confined to particular regions (Satoh, Armstrong \& Fibiger 1983; Jones 1985; Sakai 1985c). On the other hand, the majority of neurons in the brain stem reticular formation, as well as cells in the locus coeruleus and raphe nuclei, respond to acetylcholine, and these cholinoceptive neurons may respond by either excitation or inhibition (Bradley 1972). With regard to the neurotransmitters of the PS-off cells, the monoamines cannot be generally assumed to be inhibitory, since they have both inhibitory and excitatory actions on brain stem reticular neurons (Bradley 1972).

The most important question in evaluating the original model and, more fundamentally, in conceptualizing the mechanisms of state generation is whether a population of PS-specific neurons that fires selectively during PS exists, as McCarley and Hobson (1971) originally claimed, or does not exist, as Siegel and McGinty (1977) concluded for neurons of the paramedial reticular formation. Sakai (1980) has provided data indicating that such PS-specific PS-on cells exist in the dorsolateral pontine tegmentum and in the ventromedial medullary reticular formation. He has also presented evidence for a connection between these populations of potential generator neurons, results that have been supported by neuroanatomical studies of the efferent projections from the two reticular regions (Jones \& Yang 1985). Recently we have found that Kainic acid lesions of nerve cell bodies in the dorsolateral pontine tegmentum can lead to a profound disruption of paradoxical sleep (Webster \& Jones, in progress), supporting a generating role for neurons in this area. Furthermore, transection studies suggest that the interconnections between the pontine tegmentum and the medullary reticular formation are critical for the generation of the PS state (Webster, Friedman \& Jones 1986). In support of the early supposition of the role of cholinergic neurons in PS (for review, see Jouvet 1972), and the more recent emphasis on that role by Hobson and his colleagues, it has also been found that the ChAT-positive neurons of the brain stem reticular formation are concentrated in the dorsolateral pontine tegmentum and in the ventromedial medullary reticular formation (Satoh et al. 1983; Jones 1985; Sakai 1985c), in the same areas where PS-specific PS-on cells have been identified (Sakai 1985). The cholinergic (ChAT-positive) neurons (possibly PS-on cells; Sakai 1985c) in the dorsolateral pontine tegmentum have been found to be intermingled with noradrenaline (TH-positive) neurons (possibly PS-off cells; Sakai 1985c) in this region (Jones \& Beaudet, in preparation). This intermingling - which Hobson and his colleagues would choose to interpret, in perhaps too Freudian a manner, as "interpenetration" - provides a potential neuroanatomical substrate for an interaction between possible PS generator neurons and PS-off cells containing different neurotransmitters. The results of these multiple studies would allow for selective, localizable, biochemically definable neurons which interact in a specifiable, but as yet undetermined, way as playing a key role in the generation of paradoxical sleep.

In summary, the original model of McCarley and Hobson (1975b) has stimulated many experiments and new concepts in sleep research. But the original model, which was so clearly and 
logically formulated, has not been borne out, either for the involvement of the specific elements proposed or for the specific processes of interaction postulated. Changing both its elements and its assumptions, however, as Hobson and his colleagues have done in the present review, fundamentally alters the "reciprocal-interaction" model so that it appears much too general to be empirically useful, lacking in the clarity and coherence of the original. There is clearly a need for a new model of sleep cycle generation based on the precise neurophysiological and chemo-neuroanatomical data that have emerged over the past 10 years.

\section{Back to the hypothalamus: A crucial road for sleep research}

\section{Hiroshi Kawamura \\ Mitsubishi-Kasei Institute of Life Sciences, Tokyo 194, Japan}

In the past two decades, various important findings related to sleep mechanisms, especially REM sleep, in the hindbrain and particularly in the pons have been disclosed. These are without doubt valuable contributions to our knowledge of the nature of sleep. However, the question arises whether such an emphasis on the hindbrain mechanism for the induction of sleep-waking changes really matches the clinical observations accumulated for years in the fields of internal medicine and neurology. It is well-known that lesions of the posterior hypothalamus induce long-lasting irreversible comatose states with slow wave sleep ECoG (electrocorticogram) patterns. On the other hand, lesions of the bilateral preoptic region induce insomnia with lowvoltage fast $\mathrm{ECoG}$. Such clinical observations were summarized early by Economo (1930) and have been confirmed in animal experiments by Nauta (1946) and by many authors from the Magoun and Moruzzi schools.

Nevertheless, because of Bremer's classic experiment (1935) demonstrating long-lasting slow wave sleep patterns in the ECoG in the cerveau isole cat with intact hypothalamus in the forebrain, the important role played by the hypothalamus in sleep-waking alteration has virtually been ignored in recent studies of sleep mechanisms. For example, Jouvet's sleep theory (1969) fundamentally excluded the hypothalamus as a basic pacemaker for sleep-waking changes, adopting a concept of aminergic systems originating from the hindbrain in REM and non-REM sleep. Jouvet's theory was therefore controversial from the beginning because it excluded a hypothalamic role.

Of course, the contribution of the pontine area to various REM sleep signs, especially the involvement of the discrete pontine area in tonic phenomena (atonia and PGO spikes), cannot be denied. Also, for phasic REM sleep signs (rapid eye movements, muscle twitches, and bursts of neuronal discharge), Pompeiano's finding (1965) on the role of the vestibular nuclei should be taken more into consideration.

However, it has been reported that in the rostral cerveau isole preparation, if one waits for 2 or 3 days or more, low-voltage fast wave ECoG begins to reappears. This was shown by Batsel in dogs (1960) and cats (1964), by Villablanca in cats (1962;1965), and by Slozarska and Zernicki in cats (1973). Unfortunately, these investigators did not record a full 24 hours every day, so their quantitative analysis of the amount of sleep was incomplete. Moreover, the circadian rhythm in sleep-waking had not been taken into consideration.

Hanada elaborately repeated this work in cerveau isole rats with rostral midbrain transections and found that as far as forebrain $\mathrm{EcoG}$ was concerned, there was no obvious difference in the amount and duration of slow wave sleep ECoG patterns of cerveau isolé and intact rats. In the cerveau isole preparation, the locus coeruleus and almost all of the raphe nuclei were isolated from the forebrain, which showed sleep-waking changes in ECoG. Also, the circadian rhythm in sleep-waking could be found in the forebrain but was abolished after lesions of the bilateral suprachiasmatic nuclei in the hypothalamus (Hanada \& Kawamura 1981).

This work clearly showed localization of the mechanism inducing normal sleep-waking changes in the forebrain isolated from the pons and medulla. Nakata continued this work in our laboratory, lesioning either the preoptic area or the posterior hypothalamus bilaterally by passing DC current through implanted electrodes in cerveau isole rats. After lesioning the medial preoptic area she found a marked increase in the amount of low-voltage fast wave (waking) ECoG in midbrain-transected rats, whereas after lesions of the posterior hypothalamus there was an increase of slow sleep patterns approximating that of intact animals (Nakata \& Kawamura 1986). Hence hindbrain mechanisms apparently have little to do with the basic changes of slow sleep-waking indicated by the ECoG. These experiments again supported an idea that the hypothalamus, including the preoptic region, contains a specific structure or "pacemaker" of sleep-waking changes without interaction with the hindbrain. Such an observation may support an idea of reciprocal interaction in the hypothalamus to some extent, but it does not necessarily indicate the participation of specific transmitter systems originating from the hindbrain in the induction of sleep-waking changes, as had been proposed by Jouvet.

A phylogenetically old mechanism inducing slow wave sleepwakefulness changes and a circadian rhythm oscillator both reside in the hypothalamus. In intact animals these may certainly affect the newly acquired REM sleep mechanism mainly localized in the hindbrain. Probably, when some neural activity related to slow wave sleep in the forebrain reaches a certain level, it activates the "REM center" in the pontine area, inducing desynchronization of the ECoG in the forebrain by feedback through the midbrain reticular formation. Or two types of desynchronizing ECoG mechanisms may exist in the forebrain, one leading to waking and the other leading to REM sleep. Further study is necessary to resolve this question.

Summarizing all these data, sleep-waking changes camnot be ascribed to several transmitters as was initially proposed by Jouvet. After all, no sleeping pills are really pure agonists or antagonists of these substances (serotonin and norepinephrine). The cholinergic system may have an important role in the induction of REM sleep signs. Yet it is also true that the sleep mechanisms in general cannot be ascribed to a discrete cell group, especially in the pons. However, old observations concerning hypothalamic and preoptic lesions that induce coma and insomnia and concerning stimulation effects in these sites that induce arousal and sleep must be reconsidered (e.g., as Hobson described in his earlier paper 1965). This part of the brain may be crucial in clarifying the physiological sleep-waking mechanism, just as the suprachiasmatic nuclei was for understanding the induction of circadian rhythmicity.

\section{Reciprocal interaction revisited}

\section{Thomas S. Kilduff and Christian Guilleminault \\ Sleep Disorders Center, Stanford University School of Medicine, Stanford, Calit. 94305}

The emphasis on populations of cells rather than "centers" places this update of the reciprocal-interaction model by Hobson, Lydic, and Baghdoyan in the mainstream of current neurobiological thought. The authors are to be commended for attempting to move sleep physiologists away from the center concept, which has historically played an important role in sleep 
models (including the reciprocal-interaction model as originally proposed). They are also to be commended for accepting and using the REM/NREM terminology. They clearly present evidence both for and against the reciprocal-interaction model, and it is heartening to see statements such as "It is now clear that the process of REM sleep generation is far too complex to be accounted for solely by the FTG generator hypothesis." The subsequent statement that it is "premature to conclude that FTG activation . . . has no significance in the normal triggering of REM sleep" also seems fair based on the contradictory evidence in the literature to date.

Perhaps the most enduring portion of the original reciprocalinteraction model is the purported role of acetylcholine. Experimental evidence gathered since the original positing of this model has by and large supported the notion of acetylcholine in triggering at least a "REM-like" state. Recent evidence gathered by Baghdoyan and others is presented here; most intriguing is the suggestion that specific components of the REM sleep process can be elicited depending on the site of carbachol injection in the pontine region. These studies are consistent with the suggestion that a hyperactive cholinergic system underlies the REM sleep disorder narcolepsy. A proliferation of the muscarinic cholinergic receptor has been established in the brain stem of narcoleptic dogs (Boehme et al. 1984; Kilduff et al. 1986). Thus, one is left to wonder whether moment-to-moment changes in acetylcholine release or the unmasking of cholinergic receptors plays a role in the alternation between states characteristic of the sleep cycle.

Studies performed on narcoleptic dogs have also strongly implicated the amygdala and the dopamine system in the abrupt, reversible muscle paralysis (cataplexy) that mimics the atonia of REM sleep. An identical pattern of nonreciprocal motor inhibition is seen in cataplexy and REM sleep. Both Hreflexes and deep tendon reflexes are suppressed in these two conditions, and REM-sleep-suppressive drugs reduce or alleviate cataplexy. Results of an assessment of the regional neurochemistry of catecholamines, particularly homovanillic acid (HVA), 3,4-dihydroxyphenylacetic acid (DOPAC), 3-methyl-4hxdroxyphenylethyline glycol (MHPG), and 5-hydroxyindoleacetic acid (5-HIAA), from micropunches taken from discrete brain sites revealed consistent differences between normal and narcoleptic dogs (Faull et al. 1986). The most striking finding was an elevation of catecholamine and DOPAC. Dopamine, epinephrine, DOPAC, and HVA were consistently elevated in the amygdala and to a lesser degree in the rostral caudate and nucleus accumbens. These findings are in agreement with the hypothesis of a dopamine-release problem in cataplexy.

At first glance, the proposed model appears to be exceedingly complex and perhaps overly "supple" in suggesting the triggering of REM sleep at many different sites in the brain stem. Yet many other aspects of current thought on sleep mechanisms are neglected in this model. For example, the reciprocal-interaction model continues to emphasize REM sleep without adequately addressing the onset of NREM sleep. Similarly, the role of the basal forebrain and other diencephalic structures presumably involved in the initiation of sleep is not addressed. How would the suprachiasmatic nucleus fit into this scheme with regard to the timing of sleep, if at all? Given that benzodiazepines are clinically effective sleep-inducing aids, what role, if any, do benzodiazepine receptors or endogenous benzodiazepines have? What role would endogenous sleep substances or neuropeptides play in such a model? Indeed, the well-established fact of coexistence between the classical monoamine neurotransmitters and neuropeptides is barely addressed by Hobson et al. Thus, although the updated version of the reciprocal-interaction model initially appears to be too complex to be intellectually satisfying, the disconcerting news for the sleep physiologist is that this updated model may yet be too simplistic.

\section{Transmitters and REM sleep}

\section{K. Krnjević}

Departments of Physiology and Anaesthesia Research, McGill University, Montreal, Quebec H3G 1Y6, Canada

Dr. Hobson is as resourceful a writer as he is a researcher. One should therefore not be surprised that he has come up with another, by and large highly readable, major survey of sleep mechanisms. Perhaps because of its elusiveness, this topic continues to stimulate numerous and elaborate discussions.

After reading Hobson's latest contribution to this voluminous literature, however, one cannot help wondering whether it was worth the effort. For example, is it necessary to emphasize so strongly "interpenetrating neuronal populations," a concept that is now widely accepted? Similarly, I doubt that many neuroscientists still believe in global functions of the cholinergic system, this or that aminergic one. On the other hand, is there a compelling reason for stating that the sleep cycle cannot be accounted for on the basis of a single neurotransmitter?

Two crucial features of chemical transmission that have become widely recognized in the last 10 years (neither of which is mentioned in Hobson et al.'s target article) are, first, that many more endogenous neuroactive agents are present in the brain than previously suspected, and second, that any one transmitter - including the most "classical" - can have a variety of different actions, even on the same neuron. As a result, even the most expert "aminologists" would, I think, nowadays be loath to commit themselves to any definitive statement as to whether noradrenaline has a predominantly inhibitory or excitatory action in the brain. Hobson's basic assumption that aminergic pathways are simply inhibitory ignores the recent literature that describes important excitatory effects of monoamines, mediated by such mechanisms as block of $\mathrm{K}$ outward currents (M-type current and postspike hyperpolarizations).

Although peptides are no longer viewed quite so enthusiastically as putative synaptic transmitters, some may be important modulators, perhaps having a pivotal role in sleep mechanisms. At this stage can one exclude the possibility that the periodic release of a Pappenheimer/Uchizono-type peptide determines the cycling of the "coupled oscillator"?

Finally, the recent use of injections of carbachol into the brain stem - a technique that has been around for many decades, (see Hernandez-Peon in the 1960's) - hardly justifies the statement that "sleep research has come of age experimentally," especially when, in these experiments, no serious attempt has been made to demonstrate (a) on what cells carbachol is active, (b) whether these cells are excited or inhibited, and (c) that ACh is normally the principal transmitter of sleep-related signals.

Undoubtedly, successful intracellular recording in sleep studies is a major technical feat, which is presumably the basis for the repeated emphasis on the importance of "cellular and molecular mechanisms"; but one wonders whether intracellular recording can be very illuminating in the absence of a clearer idea of the multicellular organization and mechanisms and, indeed - a basic flaw - in view of the paucity of convincing evidence about the function of REM sleep. Without a better understanding of these broader issues, considerable efforts in the direction of, on the one hand, intracellular recording, or, on the other, comprehensive reviews such as this one, may not be very rewarding.

\section{The reciprocal-interaction model of sleep: $A$ look at a vigorous ten-year-old}

\section{Wallace B. Mendelson}

Section on Sleep Studies, Clinical Psychobiology Branch, National Institute of Mental Health, Bethesda, Md. 20892

A decade after the first reports of the reciprocal-interaction model of sleep regulation, Drs. Hobson, Lydic, and Baghdoyan 
have presented here a thoughtful and detailed reappraisal. It is a tribute to the flexibility of the model that it has been able to incorporate new and seemingly incompatible data. Among these have been reports that FTG. (gigantocellular tegmental fields) cell firing is as intense during certain movements in waking as in REM sleep (Siegel \& McGinty 1977) and that kainic acid lesions of the FTG cells do not prevent REM sleep (Sastre, Sakai \& Jouvet 1981; Drucker-Colin \& Bernal-Pedraza 1983). Indeed, such observations have led to one of the major reformulations of the model: that the REM-on and REM-off cells may be widely distributed in diffuse neuronal fields rather than in relatively discrete nuclei. Another major change has been the expansion of the model to include all three states of consciousness (waking, non-REM sleep, and REM sleep) rather than just the REM-non-REM cycle. This has in turn made it possible to relate the model to clinical descriptions of disorders of sleep and waking.

One of the benefits - and hazards - of expanding the range of a model is that suddenly there are many more empirical observations that it must explain. In this category are the classical reports of decreased sleep (at least in the short term) following administration of parachlorophenylalanine (e.g., Delorme 1966) or by lesions of the raphe nuclei (Jouvet, Bobillier, Pujol \& Renault 1966; Kostowski, Giacalone, Garattini \& Valzelli 1968). One would predict from the reciprocal-interaction model that any manipulation which decreased aminergic activity might lead to enhanced sleep, not wakefulness. Obviously, this might be dealt with partially by questions of the precision of the lesion studies, differences in responses to lesions of the median and dorsal raphe, and so on, but it does need to be explained. Another question arises regarding the mechanism of action of hypnotics. Agents such as benzodiazepines, which may enhance GABA-ergic activity and which have been reported to decrease activity in the raphe nuclei, would seem to fit very well with the model, assuming that their hypnotic effects are indeed mediated through a GABA-ergic mechanism (this is not yet clear). As Hobson et al. point out, however, the possible hypnotic effects of L-tryptophan (a serotonin precursor) and the tricyclic antidepressants (which block amine reuptake) are not easily explainable. In the model, such acutely enhanced serotonergic activity, or the anticholinergic activity of tricyclics, might be expected to increase wakefulness.

The effort to relate the model to clinical sleep disorders has brought out both strengths and weaknesses. In the examination of depression, Hobson et al. describe the major slccp features as being hypersomnia and decreased REM latency. If depressives were hypersomnic then reversal of symptoms with antidepressants, which (in the short term) are functionally aminergic agonists, would indeed be expected to reverse symptomatology. Most data, however, indicate that, at least with unipolar depression, patients tend to complain of insomnia and have decreased total sleep time (Gillin et al. 1984), although a small minority may experience hypersomnia (Michaelis \& Hoffmann 1973). On the other hand, the postulation of neuronal fields regulating sleep - and the observation that some REM-off fields may interpenetrate with brain stem respiratory neurons - may be an exciting development in the understanding of sleep apnea syndromes.

Finally, as Hobson et al. point out, it is important to emphasize that the movement toward models using neuronal fields rather than discrete nuclei is a step away from the localization trend that dominated neurophysiology in the 1930s and 1940s, exemplified by the work of Hess (1931) and Bremer (1935). One of the dangers of broadening models is that they may become both all-encompassing and less testable. I do not believe this to be the case here, as the authors have gone out of their way to point out predictions based on their model. It should also be added that they are only midway in a movement from the localization approach. It has been observed that thalamic neurons in brain slice studies may have two distinct firing patterns depending on the degree of baseline membrane polarization (Llinas \& Jahnsen 1982), and the analogy of this process to nonREM sleep and waking has been noted by those authors and others (McGinty 1985). This leads to the intriguing speculation that the potential for having distinct behavioral states may be an inherent property of individual neurons, existing even more diffusely than is postulated by the parents of the reciprocalinteraction model.

\section{Are cholinergic, noradrenergic, and serotonergic neurons sufficient for understanding REM sleep control?}

\section{Jaime M. Monti}

Department of Pharmacology and Therapeutics, School of Medicine, Hospital de Clinicas, Montevideo, Uruguay

I would like to comment on two aspects directly related to the reciprocal-interaction model of sleep cycle control:

(a) One of the major conclusions of Hobson et al.'s target article is that the slowing and cessation of noradrenergic (NE) and serotonergic (5-HT) cells (modulator neurons) would be necessary to release REM generator neurons from aminergic restraint. However, there is evidence indicating that NE and 5HT neurons are not necessary for the initiation and maintenance of REM sleep. Accordingly, bilateral damage to the dorsal NE bundle or the locus coeruleus (LC) by local injection of 6hydroxydopamine does not result in significant changes of tonic and phasic components of REM sleep (Lidbrink 1974; Petitjean, Sakai, Blondaux \& Jouvet 1975). More recently, DSP-4 (N-(2chloroethyl)-N-ethyl-2-bromobenzylamine), which produces a marked, selective, and long-lasting depletion of $\mathrm{NE}$ in the central nervous system, has been used to study the role of NE cells in REM sleep. Following systemic DSP-4 administration, REM sleep was decreased only transiently (Monti et al., submitted).

The 5-HT neurotoxin, 5,7-dihydroxytryptamine markedly decreases 5-HT levels in brain. In spite of that, animals have the same amounts of NREM and REM sleep (Puizillout, GaudinChazal, Sayadi \& Vigier 1981).

Recently, Jouvet and his coworkers (Sallanon, Buda, Janin \& Jouvet 1985) proposed that 5-HT released during waking induces the synthesis and/or release of hypnogenic factors which would be secondarily responsible for NREM and REM sleep.

(b) Current evidence indicates that functional interactions of NE, 5-HT, dopamine (DA), and histamine (Hist) systems occur during physiological REM sleep (Monti 1983). Thus, the inclusion of only NE and 5-HT cells as modulator neurons in Hobson et al.'s model of sleep control is rather restrictive.

The relation of pontine neurons to the striatum via multisynaptic connections (Pasquier, Kemper, Forbes \& Morgane 1977) and the recent description of DA neurons in the rostral raphe projecting to the LC (Nagatsu, Inagaki, Kondo, Karasawa \& Nagatsu 1979) and of a diencephalospinal DA system which innervates the brain stem (Skagerberg, Björklund, Lindvall \& Schmidt 1982) are suggestive of a modulatory role for DA neurons in the sleep-waking cycle. Indirect pharmacological data support this proposal (Monti 1983). In contrast to both 5 HT and NE neurons (Trulson \& Jacobs 1979) the firing rates of the substantia nigra and ventral tegmental area DA neurons are unchanged across waking, NREM sleep, and REM sleep (Trulson, Preussler \& Howell 1981). Thus, neurons that do not reciprocally interact with cholinergic cells could also be modulating the sleep cycle by yet unknown mechanisms.

The role of Hist in the control of the sleep cycle has received less attention than those of NE, DA, 5-HT, and acetylcholine. Using immunofluorescence, histamine-immunoreactive neurons were detected in the hypothalamus and premammillary 
area. Furthermore, immunoreactive nerve fibers were observed in many areas of the brain, including the cortex and some mesencephalic and pontine nuclei (Panula, Yang \& Costa 1984). H1-receptor agonists decrease NREM and REM sleep, while H1-receptor antagonists show opposite effects on NREM sleep (Monti, Pellejero, Jantos \& Pazos 1985).

In summary, a future version of the reciprocal-interaction model of sleep cycle control will have to consider DA, Yist, and probably some other modulator neurons in addition to the NE and 5-HT ones. The well-known fact that REM sleep regains control levels in the absence of NE or 5-HT neurons deserves further study.

\section{When is a "center" not a "center"? When it's "anatomically distributed": Prospects for a "diffuse REM center" ("generator")}

\section{Peter J. Morgane}

Worcester Foundation for Expenimental Biology, Shrewsbury, Mass. 01545

The trouble with brain "centers" - or, to use the electrophysiological equivalent, "generators" - is that they keep getting bigger and bigger so that one begins to wonder whether they have any limits at all. Do they eventually encompass the whole brain, or can they be subsumed in terms of recycling information in multiple feedback loops? And can "loops," as such, be "centers"? The problem with the lower brain stem is that it has an essentially reticulate type of organization, although clustering into nuclei is also clear in some areas. It generally lacks a point-to-point type of organization; hence "centering" in such a system has not been fashionable. Most areas of the lower brain stem, leaving aside the various clusters of cranial nerve nuclei and the respiratory and cardiovascular complexes, do not have a tight or nucleate type of organization. More compact organizations of cells that operate largely in particular behaviors was the older use of the term "center." Hence, diffuse elements doing the same thing means they are "anatomically distributed"; or, perhaps, we can use an even more paradoxical euphemism: "diffuse center."

Reciprocal-interaction modeling as done by Hobson and his group is really systems theory in which, instead of "nuclei" and compact centers, fiber systems interconnecting cell groups are the ones that play dominant roles in given behaviors (or in several behaviors). If there are two such major interactions (as, for example, in the thermoregulatory system) then reciprocal interaction appears to be the main mode by which they influence each other. The same holds true in the early sleep "models" of Jouvet (1969), which defined interactions between the raphe nuclei and locus coeruleus and, later on, interactions between these and an anatomically undefined cholinergic system. Once the giant cells were found by Hobson and his group to "relate" to REM sleep, Hobson's approach and theory (the reciprocal-interaction model of REM sleep) was logically the only one to take as a first approach. Where this seemed to go awry was in attempting to precisely define REM "generators" in a limited geographic region of the brain stem. So now Hobson's view of "distributed centers" or "expanded centers" or, to use his term, "populations" has made some older concepts of "sleep systems" even more relevant in thinking about how complex behavior such as REM sleep is generated. Thus, in dissecting a behavior we have come to think in terms of diffuse populations of neurons as well as the multitude of chemically coded pathways that interlink them. Obviously, "expanded center" or "population" or "diffuse center" is a somewhat paradoxical use of the word "center." The term has lost its original meaning, even though "generators" of behavior are precisely what a "center" originally implied in the heyday of subcortical phrenology.
Leaving these kinds of arguments aside, who can really dispute that multiple reciprocal interactions are one major means by which brains operate? The problem is putting some "limit" on how far we should expand a functional system. Obviously, a whole functional system involves the whole brain. Given that many integrative "levels" participate in complex behavior and that none of these is "pure" in terms of chemical coding (one-amine/one-behavior views went by the boards almost immediately after amine systems began to be correlated with specific behaviors), what, then, is really unique or heuristically valuable is the reciprocal-interaction model(s) of Hobson and colleagues even in its newly extended form? As originally set up the model was neat, orderly, and logical, but it was far too simple and "local" to account for the complex known as REM sleep. Simply put, not enough "levels" in the nervous system were examined by Hobson or others to really establish how expanded or "diffuse" the REM sleep system might be. Now that a more expansive view of the REM universe has been taken by Hobson it does, on paper, appear to provide a more viable substrate for complex behavior. However, examining "levels" of function in the integrative sense of Sherrington has never been adequately done either with purely motor functions or with any other complex behavior, and, accordingly, one may wonder how likely such approaches are to have success in understanding REM sleep. There is little doubt that such wider approaches make more overall sense than continuing exhaustively (and exclusively) to examine local regions of the brain. The real problem is that such integrative approaches are just too difficult technically for present-day analyses.

So the problem laid out here is really a very old one dressed in some new clothing: how to examine the brain over wide areas (as a whole or as a series of integrative levels) rather than in the easier-to-study "centers" and local cell conglomerates. Every field of integrative behavior has reached such a stage, including feeding behavior, thermoregulatory behavior, sexual behavior, among others; and, for practical reasons, we have tended to return as to a refrain to more elemental views of cell clusters as "functional centers" for the very reason that such groups lend themselves to more orderly analysis.

What appears to have happened here is that after years of good, solid electrophysiological analyses of the brain stem we are not really much nearer to a definitive view of REM generation in terms of modes of interrelations of brain "levels" or activity in chemical systems that link them. Hobson and his team can be congratulated for being among the best at what they do, but electrophysiological investigations in themselves have severe limits and cannot provide anything approaching ultimate answers to many of the questions they pose.

So where do we go from here? How will Hobson and his group, or anyone, know how far to expand a "center"? And what really defines its limits? Can an expanded or "diffuse" center be only another relatively equipotential view of the neural universe? How "relative" are the roles of brain areas and "levels" in complex functions and in the organizion of sequential behaviors? How is that "relativity" established? And, since areas and transmitters play roles in many behaviors, how can we get to a realistic view of the exact role of chemical pathways in specific behaviors? Furthermore, what about the participation of the putative 50- to 100-or-so-transmitter systems in brain function? Presumably the maker of brains did not put them in to serve as some sort of scaffolding. Does "chemical interpenetration," to use the Hobson phrase, say the obvious: that amine and other chemically coded neuron clusters are not pure cultures containing specific transmitters? Of course, cotransmitters make these models even more complicated to unravel in terms of simple interaction theory.

Now to some specifics! I would definitely not agree with the authors' statement that "until recently, the states of waking and sleep were studied within the paradigm of research on neural centers." Much previous work was actually not done within a 
paradigm of neural centers. For example, Hernández-Peón and coworkers (Hernández-Peón, Chavez-Ibarra, Morgane \& TimoIaria 1962; 1963) pharmacologically "mapped" (using cholinergic stimulation) widespread neural systems across the brain from the septum to the limbic midbrain area showing clear and unambiguous progression of animals from waking through slow sleep and into a REM-like state with desynchronized cortex, muscle atonia, rapid eye movements, and hippocampal theta activity. This may not have been "physiological" REM sleep, but it was definitely a REM-like state with all the relevant signs. Furthermore, Jouvet (1969), in setting up his original chemical models of the sleep states, described reciprocal interactions between the raphe nuclei and locus coeruleus and even interrelated cholinergic systems in this model (see Figure 7, p. 38). The Ruch-Monachon, Jalfre \& Haefely (1976) series of chemical model systems of PGO spike generation as clearly examining interacting chemical systems (see their model on p. 340) in the regulation of $\mathrm{PGO}$ spike activity. None of these investigators was really working within paradigms of neural centers.

What does the Hobson group mean by the phrase "unique behavioral-state-specific discharge patterns" (italics mine)? Is it really "unique" until other levels of integration are examined and many other putative REM onset cells are mapped in the "expanded-population" approach? Is it not premature to speak of "uniqueness" given the new extended-population view?

On the conceptual side, I am not convinced that the general concepts of the "reciprocal-interaction" models of REM sleep can really be refuted. Reciprocal interaction seems to be one main organizing principle of the CNS. When multiple complexes of reciprocal interaction are examined across several "levels" of integration, can they realistically be assessed as necessary and sufficient for playing a role in organizing a complexing behavior? Can they serve as a "model of initiation or generation of a behavioral and electrographic complex such as REM sleep"? Has any complex behavior been shown to have such a "generator" clustered in the interaction of interpenetrated neuron assemblies, including when part of the complex operates as a "brake" ("permissive," section II, first paragraph), such as amine systems acting on FTG neurons (see also the literature on feeding behavior, where for many years it was fashionable to use the concept of a "satiety brake" [ventromedial nucleus of the hypothalamus] acting to directly inhibit a feeding "center" or "generator" located in the lateral hypothalamic area)? In addition, since most cell groups appear to have multiple regulatory roles how is it possible to "dissect" such roles in specific behaviors?

I also wonder why Hobson et al. are surprised that the cholinergic/cholinoceptive system (part of the proposed oscillator) is also "less likely to be localized." Hernández-Peón et al. $(1962 ; 1963)$, using chemical stimulation of the brain, showed "cholinergic sleep" in many areas of the medial forebrain bundle system and limbic-midbrain circuit extending to the dorsal nucleus of Gudden in the brain stem. This was really an "extended sleep system" if there ever was one! By not referring to those key early and important studies, Hobson falls into a trap in section II.A.l.a, paragraph 7, in stating that the "anterodorsal pontine tegmentum is the only brainstem reticular site from which a behavioral state resembling REM sleep has been induced pharmacologically" (italics mine).

In section II.A.1, paragraph 2, the authors note that a "vast ensemble of [REM-on] cells constitutes the population that finally acts as effectors of the physiological signs of REM sleep"? They go on to state that "it is now clear that REM sleep generation is far too complex to be accounted for solely by the FTG generator hypothesis." If, as stated in section II.A.1.a, paragraph 3, "the FTG is only one of a set of upper motor neuronal systems to be activated in REM sleep" (italics mine), where does this really leave FTG neurons in the big picture? Why would they still be more viable candidates than other putative REM "generators"?
Then we come to the statement in the following paragraph that "it is clearly premature to conclude that FTG activation either is entirely movement-related or has no significance in the normal triggering of REM sleep" (italics mine). Though such a statement may throw one off stride, the key words are "entirely" and "has no significance," both of which state only extremes. Why not use terms such as "definitely, but not exclusively movement-related" and "have limited or partial significance"? Since none of this is presently "black-and-white" in any sense, I would take exception to going totally defensive with a statement like the one above; no one really has said that FTG activation is "entirely" movement-related or has "no significance."

I do not think the hypothesis of causality (that a specific cellular population causes a behavioral state) can be accepted with a high degree of confidence: Unfortunately, much of it is still in realm of correlation. What does it really mean in section II.A.1.a, paragraph 6, that "REM sleep is in part caused by FTG discharge" (italics mine)? In section II, paragraph 2, Hobson states that REM-on cells, "anatomically... distributed in the brain stem . . . play a 'key role' in generating REM sleep [behavioral state control]." How can it be a "key role" if, when most of the FTG cells are destroyed, the main elements of REM sleep can still occur? Furthermore, I wonder why it is stated in section II.A. 1.a, paragraph 5, that "FTG cells are neither necessary nor sufficient for initiating REM sleep"? If not, what does this do to the view of the "key role" of FTG cells in REM sleep? Is this model, even in expanded form, still rich in predictive powers? I have some doubts. The original model did its thing, was called into question, and has thus been developed into a broader view of the organization of complex behaviors. It can still play limited roles in serving as a model for other subcomponents of the extended REM mechanism. But can a "model" keep getting larger and larger and still be productively examined? Finally, Hobson and his group do elegant neurophysiology and have been major contributors to the field by subjecting cell clusters in the lower brain stem to exquisite quantitative physiological analysis. However, based on everything we can guess about how brains may eventually turn out to work, to make their views of "extended reciprocal interaction" the "centerpiece" of sleep biology may not be in order at this time.

\section{Proposed model of postural atonia in a decerebrate cat}

\section{S. Mori and Y. Ohta}

Department of Physiology, Asahikawa Medical College, Asahikawa 078-11, Japan

For the past several years, our group has been studying the cellular basis of postural atonia in the decerebrate standing cat (Mori, Sakamoto \& Ohta 1986; Sakamoto, Atsuta \& Mori 1986). We have also made studies of atonia in chronic freely moving cats (Mori et al. 1986). Our findings may therefore be relevant to an understanding of the generation of atonia during sleep.

Experimental preparation. Following precollicular-postmammillary decerebration, cats were placed in a stereotaxic apparatus over a moving surface that could be activated to induce walking when the mesencephalic locomotor region (MLR) was electrically stimulated. We could also record hindlimb force when the surface was still. We delivered electrical stimuli to the brain stem through fine-tipped carbon microelectrodes and observed the effects on locomotion and/or hindlimb force.

Induction of atonla. Stimulation of the midline at the pontine brain stem between P3 and P7 (Horsley-Clarke coordinates) at a depth of 2-4 $\mathrm{mm}$ below the surface of fourth ventricle produces an interruption of ongoing locomotion and/or a dramatic collapse of hindlimb tonus that outlasts the stimulation by many 


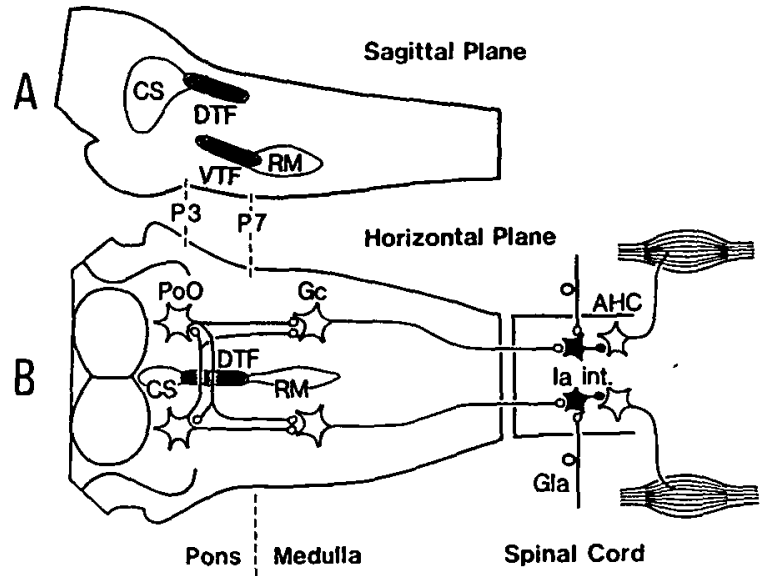

Figure 1. (Mori and Ohta). Schematic diagrams of the locations of the effective areas inducing atonia and hypertonia on the midline sagittal plane of the brain stem (A), and of the proposed neuronal network inducing atonia on the horizontal plane of the brain stem and the spinal cord. DTF, dorsal tegmental field; VTF, ventral tegmental field; AHC, anterior horn cell; CS, nucleus centralis superior; $G c$, nucleus reticularis gigantocellularis; GIa, group Ia muscle afferent; Ia int, Ia interneuron; $\mathrm{PoO}$, nucleus reticularis pontine oralis; $\mathrm{RM}$, nucleus raphe magnus.

minutes. Of particular interest to the model proposed by Hobson et al. is the fact that the effective zone, which we call the dorsal tegmental field(DTF), lies between the caudal pole of the nucleus raphe centralis superior and the cephalad pole of the nucleus raphe magnus (Figure 1A). This area is sparsely populated by neurones but is densely packed by fibers connecting the two paramedian reticular giant cellular fields. The suggestion that the effects may be mediated by activation of the pontine reticular formation is supported by our finding that the microinjection of carbachol into the PRF also produces atonia, a result that confirms the findings of the Hobson group. In the presence of carbachol, electrical stimulation of a deeper pontine midline region called the ventral tegmental field (VTF), which normally produces an enhancement if hindlimb tonus (or an acceleration of ongoing locomotion), is ineffective.

Both atonia (following stimulation of the DTF) and hypertonia (following stimulation of the VTF area) were also observed in chronically prepared freely moving cats. We have not observed any signs of sleep, nor have we yet made systematic attempts to induce or enhance the atonia of REM by prolonged stimulation of the DTF.

Cellular analysis of postural atonia. Using a combination of intracellular recording (from antidromically identified spinal neurones), microstimulation, and spike-triggered averaging, we have been able to establish a disynaptic pathway from the DTF in the pons, via the gigantocellular nucleus in the medulla and the Ia inhibitory interneurones, to the anterior horn cells of the spinal cord (Figure 1B).

A period of DTF stimulation ( 5 to $10 \mathrm{sec}$ ) immediately interrupts spontaneous spike discharge and produces steplike graded membrane hyperpolarization of extensor alpha motoneurones (Sakamoto et al. 1986). Spontaneously occurring spikes in the gigantocellular nucleus of the medulla, whose cells may be identified antidromically as projecting to the spinal cord, are followed by disynaptic IPSPs (inhibitors postsynaptic potentials) in impaled alpha motoneurones and can be driven by microstimulation of the DTF (Ohta, Sakamoto \& Mori 1985; Ohta, Nonaka \& Mori 1985). The results are comparable with the concepts of atonia by Hobson et al. but suggest a modification of the schematic of their Figure 1 to include Ia interneurones at the level of the spinal cord as well as an excitatory synapse between the pontine and medullary reticular nucleus.
We do not yet know the identity of the cells of origin of these effects in the pons, but our own HRP (horseradish peroxidase) studies of the DTF favor the interpretation, advanced above, that microstimulation to the DTF area activates paramedian pontine reticular neurones, which in turn activate the gigantocellular cells of the medulla. This is because HRP, injected into DTF microlesions, is transported both orthogradely and retrogradely into the paramedian pontomedullary reticular formation with an impressive density (Ohta, Sakamoto \& Mori 1984).

Conclusion. It is impossible to know whether the effects we have observed in decerebrate cats are the same as those which mediate the atonia of REM sleep, but all the available evidence favors an identity of mechanisms; and yet there is also evidence against this (Ohta et al. 1985).

This hypothesis could be further strengthened by an application of our techniques to the study of sleep and an application of the techniques developed by Hobson et al. to our preparation. For example, we would predict that microstimulation of the DTF would monosynaptically and/or orthodromically activate pontomedullary FTG neurones. As a consequence, it might also be expected to trigger PGO waves, eye movements, and EMG (electromyogram) suppressions; it might even shorten the latency to REM sleep onset if delivered in non-REM sleep in intact cats. By contrast, microinjection of neostigmine into the pontine FTG should produce atonia or interrupt ongoing locomotion in the decerebrate standing cat, indicating that DTF effects, like REM sleep atonia, may be cholinergically mediated. And because many of the effects of MLR stimulation are thought to be mediated by aminergic modulation of spinal cord excitability, the interaction of these systems with apparently cholinergic mediation of DTF effects warrants investigation.

In any case, the two approaches and their findings are at least complementary and allow us to anticipate further mutual enrichment of the domains of motor systems and state control neurobiology.

\section{Vasotocin: Neurohumoral control of the reciprocal-interaction model?}

\section{J. R. Normanton}

University Laboratory of Physiology, University of Oxford, Oxford OX1 3PT, England

Of some interest in Hobson, Lydic, and Baghdoyan's reappraisal of the reciprocal-interaction model is their inclusion of the dorsal raphe nucleus (DRN) in their definition of a REM-off neuronal population and the importance they appear to attach to it. They suggest that in terms of discharge patterns the DRN shows "the most impressive REM-off selectivity" and that the DRN "plays a causal role in modulating the temporal organization of REM sleep." However, they acknowledge that their model "has no way of accounting for the observed refractoriness of DRN cells to excitatory stimuli during REM sleep," and the model appears to offer little explanation of how circadian temporal profiles of the sleep-waking cycle are controlled or change during ontogenetic development.

In this context I feel it essential to draw attention to some of the work carried out on one of the so-called sleep peptides, arginine-vasotocin (AVT). AVT has been found localized to the mammalian pineal gland (Bowie \& Herbert 1976; Fernstrom, Fisher, Cusak, \& Gillis 1980), from which it has been shown to be released diurnally and in a REM-dependent manner in man (Pavel, Goldstein, Popoviciu, Corfariu, Foldes \& Farkas 1979). Its sleep-inducing actions (Pavel, Psatta \& Goldstein 1977), which include REM sleep enhancement in man (Coculescu, Serbănescu \& Temeli 1979; Pavel, Goldstein, Petrescu \& Popa 1981), are mediated via activation of neurones whose cell bodies 
lie in the lateral habenula nucleus (LHB) and which give rise to an LHB-DRN pathway in which there is a GABA-ergic link (Pavel \& Eisner 1984). This pathway has been proposed by others as a major link between the forebrain and the raphe nuclei (Aghajanian \& Wang 1977; Wang \& Aghajanian 1977a). Pinealectomy (Mouret, Coindet \& Couvet 1974) and injection of specific AVT antisera (Pavel \& Goldstein 1981) have been shown to induce a narcolepticlike distribution of REM sleep, with profound but reversible effects on both NREM and REM sleep being noted following LHB lesions (Goldstein 1983a). Early work carried out in my own laboratory showed that AVT, applied by microiontophoresis, could induce profound, longlasting (up to $10 \mathrm{~min}$ ) excitations of central neurones followed by long-duration (50-90 min) desensitization (Normanton \& Gent 1983); more recently such observations have also been noted in the LHB (Normanton \& Lovick, unpublished data). A DRNpineal inhibitory pathway has been demonstrated (Léger, Degueurce, Lundberg, Pujol \& Møllgård 1983), leading to the suggestion that the pineal gland may be controlled, at least in part, by a tonic inhibitory input from the DRN. In addition, an excitatory LHB-pineal pathway has been demonstrated (Rønnekleiv, Kelly \& Wuttke 1980). The surprisingly low doses of exogenously applied peptide $(\sim 10-6 \mathrm{pg})$ required for AVT's sleep-inducing effects (Pavel et al. 1977) has led to speculation that such a dose may represent only the trigger for the release of physiologically significant amounts of AVT, possibly via feedback systems using one or both of these pathways. In support of this, AVT appears to be largely ineffective when applied any. where other than in the pineal recess adjacent to the LHB and the pineal gland itself (Goldstein 1983b), which suggests either an intrapineal or an LHB-pineal positive feedback system. However, AVT-mediated activation of the LHB-DRN pathway would result in inhibition of DRN neurones, which might in turn promote additional AVT release by disinhibition of the pineal. Irrespective of the precise mechanism(s) governing AVT release, it seems likely that eventual termination of such a process could then be brought about by desensitization of the habenula neurones to the effects of AVT with the loop remaining inoperative for the duration of such desensitization.

It is therefore tempting to speculate at this stage that at least some of the temporal aspects of REM sleep organization and of DRN discharge profiles may reflect the dynamics of such a secondary DRN-pineal-LHB-DRN loop the activity of which is expressed by way of interaction with the DRN component of the reciprocal-interaction model.

Several factors make this hypothesis a particularly attractive extension to the reciprocal-interaction model. First, developmental changes in pineal function parallel changes in the temporal organization of REM sleep with age (Roffwag, Muzio \& Dement 1966). Pineal and cerebrospinal fluid AVT (Pavel 1978; 1980) and the extent of the secretory pineal ependymal cells (Palkovits, Inke \& Lukács 1962) decrease at the same rate as the ontogenetic decrease in REM sleep. Second, the time course of AVT's actions on single neurones and its attendant desensitization approximately parallel the average duration of REM episodes and the inter-REM period respectively. Third, the hypothesis explains the apparent refractoriness of raphe neurones to excitatory stimuli during REM episodes in terms of a powerful inhibition of DRN neurones by activation of the LHB-DRN inhibitory pathway. Finally, circadian control of the sleep state oscillator may be explained at least in part in terms of modulation of this DRN-pineal-LHB-DRN loop. The pineal gland itself is under the direct control of the suprachiasmatic nucleus expressed by a way of modulation of the synthesis and secretion of melatonin (Klein 1983), which has been suggested as the physiological releasing factor for AVT (Pavel 1979). Furthermore, taking the example of vasopressin cited by the authors, vasopressin-containing fibres and terminals, arising from cell bodies located in the SCN, have been observed with particularly high density in the LHB (Sofroniew 1983); and we have recently shown that vasopressin causes inhibition of some LHB neurones when applied by microiontophoresis (Normanton, Flitcroft \& Lovick, unpublished data). Changing levels of CSF vasopressin or discharge in vasopressinergic hypothalamo-LHB pathways could therefore modulate the gain of the putative positive feedback system for AVT release and/or the effectiveness of AVT in activating the LHB-DRN pathway.

I hope that this commentary has illustrated that additional complex interactions in other quite distinct neuronal populations, which express their output through and in turn are controlled by the elements of the reciprocal-interaction model, may need to be considered in order to fully understand the dynamics of the sleep-waking cycle and place the reciprocalinteraction model in its true physiological context.

\section{On the significance of the revised reciprocal-interaction model}

\section{K. Sakai}

Département de Médecine Expérimentale, Université Claude Bernard, 69373 Lyon Cedex 08, France

I was greatly impressed by the heuristic and instructive value of Hobson, Lydic, and Baghdoyan's target article about the neural regulation of the mammalian sleep cycle. I have no intention of presenting criticisms regarding the details of this presentation. Instead, I shall briefly comment on some points where I am not in agreement with the authors.

A basic question that arises in reading Hobson et al.'s paper is: What is the concept of REM-on (or PS-on), cells in the revised reciprocal-interaction model? This revised model is obviously less precise and explicit than the initial formulation of the model because of the ill-defined notion of REM-on cells. In the original model, REM-on cells corresponded to "putatively cholinergic FTG neurons," or "REM phenomena generator neurons," that constituted a "REM generator neuronal population." In this context the hypotheses on reciprocal interactions between FTG neurons and monoaminergic neurons were meaningful. In the revised model, however, REM-on cells are described merely as tonic and phasic units whose discharge rate increases in REM sleep, for example, "cell groups . . . discharge during rapideye-movement (REM) sleep (REM-on)," "some neurons discharge more (REM-on cells) and some neurons cease discharging (REM-off cells)," "the putatively cholinergic/cholinoceptive REM-on cell network is widespread and its component neurons at the cortical, brain stem, and spinal cord levels," and so on. In other words, the minimal criteria for being REM generator neurons, such as "selectivity" and "tonicity," are neglected in the revised reciprocal-interaction model. This should be a critical and basic problem for this model.

I agree with Hobson et al. that REM sleep or PS is not generated by a single and highly localized neuronal population, but I do not agree with them when they assert that PS generator neurons responsible for the initiation and maintenance of this state of sleep are widespread and that "any localization of a putative REM sleep generator even to the brain stem could, theoretically, be an illusion." Even though the selectivity and tonicity characteristics of the FTC neurons have been refuted, it cannot be ruled out that such cell groups are present in other regions of the brain stem. Indeed, as first reported by Netick, Orem \& Dement (1977) in head-restrained cats, and subsequently confirmed and extended in freely moving cats by us (Sakai, Kanamori \& Jouvet 1979; Kanamori, Sakai \& Jouvet 1980; Sakai 1985a), "PS-on cells" characterized by tonicdischarge, highly selective (infinite discharge ratio for PS/waking) for the periods of PS really exist in certain brain stem structures: for example, the nuclei locus coeruleus (LC) alpha and peri-LC alpha in the mediodorsal pontine tegmentum and 
the nuclei reticularis magnocellularis (Mc) and parvocellularis $(\mathrm{Pc})$ in the bulbar reticular formation. It is important to mention that recent immunohistochemical studies using monoclonal antibodies for choline acetyltransferase (ChAT) have demonstrated in the cat the presence of ChAT immunoreactive neurons in these pontomedullary regions, whereas, as in the rat, they failed to reveal ChAT immunoreactive cells in the gigantocellular tegmental field or FTG (Jones 1985a; Sakai 1985b). Furthermore, reciprocal connections between the pontomedullary neurons and monoaminergic pontomedullary ones have been demonstrated, although there is, for the moment, no direct evidence of a neuron-to-neuron connection (cf. Sakai 1985a). Lesion and brain transsection experiments, as well as cholinergic microinjection studies (see below) also appear to support the hypothesis that PS is generated not by a single neuronal population but by several highly localized ones. I think that this hypothesis is still heuristically useful and I believe it indispensable to specify and localize, as much as possible, the neuronal populations responsible for each phenomenon of PS ("PGO-on cells," "atonia-on cells," "rapid eye movement-on cells," "neocortical EEG desynchronization-on cells," etc.) and also those responsible for triggering and maintaining the state of PS, that is, "PS-on cells." For more rigorous tests and better reciprocal-interaction hypotheses, a prerequisite would be to put forward an operational and theoretical definition of the concept of REM-on cells.

Relevant to this issue is the problem of the anatomical terminology. Although Hobson et al. state that "Berman's stereotaxic atlas is a standard reference found in virtually every neurophysiological laboratory," his nomenclature is unfortunately unsatisfactory, particularly when it comes to the dorsal pontine tegmentum, which contains key structures for the reciprocalinteraction hypothesis. For example, Berman includes in the nucleus locus coeruleus (LC), so important in the reciprocalinteraction model, both the aminergic $\mathrm{LC}$ and the cholinergic laterodorsal tegmental nucleus (Ldt) of Castaldi. In addition, the boundary of the reticular portion of the nucleus (nucleus locus coeruleus pars alpha) is ill defined so that this region is hardly distinguished from neighboring FTP (paralemniscal tegmental field), FTL (lateral tegmental field), FTC (central tegmental field), and even FTG (gigantocellular tegmental field). In order to avoid pointless debate, "anterodorsal pontine tegmental regions," so critical in the induction a of REM-sleep-like state by cholinomimetic microinjections, should be anatomically defined. For the evaluation of the reciprocal-interaction model, based on cholinergic REM-on and monoaminergic REM-off interactions, it is now a minimal prerequisite to determine, as exactly as possible, the neuronal subgroups in some particular areas of the brain stem.

A remaining key question concerns the concept of the reciprocal-interaction model. According to the original and revised reciprocal-interaction model, REM-on cells are excitatory to REM-off cells, whereas REM-off cells are inhibitory to REM-on cells. What is more, arrest of firing by monoaminergic REM-off cells disinhibits cholinergic REM-on cells, and this process is critical for initiating the REM sleep episode. In other words, monoaminergic REM-off cells play the role of trigger or prime mover for the production and maintenance of REM sleep, and in this sense, among others, the reciprocal-interaction model proposed by Hobson and McCarley is different from my tentative model (cf. Figure 3B). According to my model, which is still too open and therefore less refined, PS-off cells are inhibitory to PS-on cells, but PS-on cells are also inhibitory to PS-off cells. Accordingly, the state of PS can occur either by direct excitation of PS-on cells or by inhibition of PS-off cells, and an exactly inverse relationship in terms of cellular discharge is present between PS-on and PS-off cells throughout the sleepwaking cycle (Sakai 1985b).

In a series of cholinergic microinjection experiments, Hobson and his coworkers have emphasized that the "anterodorsal pontine tegmentum" is the only brain stem reticular site from which a behavioral state resembling REM sleep has been induced pharmacologically. The results would suggest, on the one hand, the possible existence of highly localized PS generator neuronal populations and would imply, on the other hand, that the state of PS can be induced by a direct excitation of PS-on cells. Although Hobson and his colleagues have asserted that the most effective injection site of carbachol corresponds to the rostral FTG, their results (cf. Silberman, Vivaldi, Casfield, McCarley \& Hobson 1980, Figure 3) seem to indicate clearly that the peri-LC alpha and adjacent LC alpha regions, where putatively cholinergic PS-on and putatively monoaminergic PSoff cells interpenetrate, play the most important role in the pharmacological induction of PS. If REM-on cells exert an excitatory influence on REM-off cells and if carbachol excites noradrenergic REM-off cells in unanesthetized freely moving cats, do the REM-off cells remain active during the pharmacologically induced REM-like state? I would imagine that during such a state of PS, PS-off cells would stop firing so that the direct excitation of PS-on cells would lead to the inhibition of PS-off cells. This and other differences between the two models will be tested experimentally.

The mechanisms involved in the occurrence of PS could be much more complicated than can be explained by simple cholinergic PS-on and monoaminergic PS-off neuronal interactions. Nevertheless, I thank Hobson et al. for providing us with a timely and heuristic presentation that is undoubtedly useful for a better understanding of cellular mechanisms underlying the genesis of PS.

\section{Is there a choice in "Hobson's choice"?}

\section{Arnold B. Scheibel}

Departments of Anatomy and Psychiatry and Brain Research Institute, UCLA School of Medicine Los Angeles, Calit. 90024

In early 17th-century England, Thomas Hobson, a CambridgeLondon liveryman, became famous for his particular method of renting out his horses. The potential customer could only take the horse whose turn was next up. Hobson apparently loved his animals and carefully rotated them through their chores - so any animal that the customer "chose" was, in every sense, Hobson's choice.

Allan Hobson, with more than a decade of elegant work on reticular physiology behind him, has offered us another version of such a choice, and, unlike a number of doughty English travelers, I find the new selection attractive and appropriate. Put in a somewhat different way, I feel he is offering us the only real horse in the stable, all other conceptual possibilities more probably being sheep or goats. He argues persuasively for "anatomically distributed and neurochemically interpenetrated populations of cells" forming the substrate for the "putative sleep cycle oscillator." The histology of the system tells the same story. Sensitive Golgi impregnations, especially in small immature animals where there is still some hope of visually dissecting a few elements from the complex neuropil, provide what may yet be the best evidence. The majority of reticular axons, no matter what their nucleus of origin, seem to generate innumerable collaterals along the way. At one time we estimated one collateral emerging every $100 \mu \mathrm{m}$ along the axonal course (Scheibel \& Scheibel 1958). Some were short, perhaps no more than $100 \mu \mathrm{m}$, leaving the axon at right angles and generating, as we saw it at the time, a cylindrical "zone of potential interaction" along the entire course of the axon. Other collaterals were longer, penetrating deeply into surrounding cell and fiber forests. Metaphorically speaking, reticular neurons in general seemed both inquisitive and talkative. They were "fence hangers" prone to gossip along the way. Allan Hobson's conceptual 
progress from neuronal center to neuronal populations, at once thematically focused and broadly interactive, is not problematic in this type of setting.

Yet, even richer structural paradigms are now known to exist which may be cited as substrates for the model. It seems clear that those dendrites, stretching for hundreds of microns in every direction, do more than simply penetrate into other nuclear fields. With maturation, they are apparently resculpted and grouped into dendrite bundles. Small sheaths of dendrites, frequently from cell bodies in different nuclei, now run together, shaft to shaft, with only a few tens of angstra of "space" between them (Scheibel, Davies \& Scheibel 1973). The functional role of these aggregates is still uncertain (Roney, Scheibel \& Shaw 1979), but it is hard to believe that these long stretches of multielement membrane apposition are not adding to the physiologic repertoire of reticular interaction.

Undoubtedly significant, too, are the rich systems of dendrovascular apposition which are now known to characterize some of the aminergic nuclei of the reticular core. Since the original description in elements of the raphe nuclei (Scheibel, Tomiyasu \& Scheibel 1975), this structural complex has proven an intriguing and frustrating addition to our knowledge of substrate systems in the core. Whether it will provide a neurohumoral system for communication - between reticular core elements, or with more distant targets - remains an intuitively attractive question, but the evidence is still lacking.

In any case, the histological emphasis is on richness and heterogeneity of structural apposition and synaptic interaction. An axon may nominally proceed from $A$ to $J$ but communicates, to visual inspection at least, with intervening elements $B$ through I along the way. If we then add to this classic communication paradigm the potential wealth of dendrite-to-dendrite communication, and perhaps even a neurovascular channel to boot, the proposed model of interactive neuronal populations appears less an attractive possibility than a necessity. It also underlines the difficulties Hobson cites in drawing meaningful conclusions from "localized" surgical lesions. The site of damage may be discrete, but the elements destroyed are almost certainly of many sources. If microinjection technology has progressed to the point where "magic bullets" will activate or destroy specific cell populations or even specific receptor sites on such cells, then we have come to halcyon times indeed.

So history repeats itself, admittedly in very different reference frames. Three hundred fifty years ago, Hobson's choice was really no choice at all. It followed rationally and inevitably from the squire's essential devotion and concern for the labor of his animals. The same can be said for (Allan) Hobson's choice today. His functional model grows inevitably from the substrate in which it is grounded. And so it's not really a choice either.

\section{The REM generator: Here, there, and everywhere?}

\section{Priyattam J. Shiromani and J. Christian Gillin \\ Department of Psychiatry, University of California, San Diego, and Veterans Administration Medical Center, San Diego, Calif. 92093}

The central tenet of the original Hobson \& McCarley reciprocalinteraction theory (McCarley \& Hobson 1975b) was that increased activity of a localized group of cholinergic/cholinoceptive cells was instrumental in REM sleep generation. This theory was based on evidence derived from singleunit studies conducted in head-restrained cats. However, in freely behaving cats a localized group of pontine reticular formation (PRF) cells showing increased discharge selectively during REM sleep has not been found (Siegel \& McGinty 1977), and kainic acid lesions of the medial PRF area hypothesized to contain neurons critical to REM sleep generation do not abolish REM sleep (Drucker-Colin \& Bernal-Pedraza 1983; Sastre,
Sakai \& Jouvet 1981). These findings forced a reevaluation of the reciprocal-interaction model, and it is currently hypothesized that a diffuse PRF mechanism is responsible for REM sleep generation (McGinty 1985). Hobson and his coworkers now support this view.

Hobson et al. continue to postulate that a reciprocal interaction between "REM-off" and "REM-on" cells is instrumental to REM generation and that certain medial PRF areas (e.g., the gigantocellular' tegmental field or FTG) contain more "REMon" than "REM-off" cells. However, their notion of interpenetration and selectivity (summarized in Figure 2) is only partially supported by evidence from immunohistochemical and single-unit studies. For example, in the FTG, Hobson et al. postulate that putative cholinergic-cholinoceptive REM-on cells outnumber REM-off cells by a ratio of 10:1 (see Figure 2C). However, it has been shown that the FTG contains very few muscarinic receptors (Wamsley, Lewis, Young \& Kuhar 1981) and only a few scattered choline acetyltransferase (ChAT) containing cell bodies (Kimura \& Maeda 1982; Kimura, McGeer, Peng \& McGeer 1981). In fact, the dorsolateral pontine tegmentum, a region where more REM-off cells are located, contains a higher proportion of cholinergic-cholinoceptive cells than the FTG (see Kimura et al. 1981, Figures 71-80). Therefore, the FTG does not contain the number of excitatory cholinergiccholinoceptive cells hypothesized in Figure 2.

In order to better understand whether electrophysiologically identified REM-on cells are cholinergic we (Shiromani, Armstrong, Groves \& Gillin 1986) have begun to examine the relationship of REM-on cells to cells staining positively for ChAT. Using the extracellular recording method we monitored the sleep-related discharge rates of 49 neurons ventral to the locus coeruleus and medial to the motor nucleus of the fifth nerve ( $\mathrm{AP}=-2$ to $-4 ; \mathrm{L}=2.5-3.0 ; \mathrm{H}=2.5$ ). This area is slightly lateral to the PRF area where carbachol readily evokes REM sleep (Baghdoyan, Rodrigo-Angulo, McCarley \& Hobson 1984). Nevertheless, Jones and her colleagues (Friedman \& Jones 1984) have recently shown that electrolytic lesion of the region ventral to the locus coeruleus and medial to the motor nucleus of five, in cats, completely abolishes REM sleep. We found that some cells in this region displayed a general increase in discharge rate during REM sleep ( $8 \%$ of the cells showed a progressive linear increase from waking to REM sleep, and $24.5 \%$ showed their highest discharge rate during waking and REM sleep). Preliminary examination of brain stem tissue incubated in polylonal ChAT antibody revealed a lack of cholinergic cells in this region. However, muscarinic receptor binding (Wamsley et al. 1981) and pharmacological infusion studies (Baghdoyan, Rodrigo-Angulo, McCarley \& Hobson 1984; Shiromani 1983; Shiromani \& McGinty 1983; 1986; Shiromani et al. 1986) have shown this region to be cholinoceptive. Our findings therefore lead us to believe that cholinoceptive rather than cholinergic neurons in the PRF may be instrumental to REM sleep generation. The cholinoceptive neurons may in turn project to the dorsolateral pontine tegmentum cholinergic cells, located principally in the lateral-dorsal tegmental (LDTn) and pedunculopontine tegmental (PPTn) nuclei, which give rise to the dorsal cholinergic pathway (Wilson 1985). This pathway projects to the thalamus, hippocampus, hypothalamus, and neocortex (Mesulam, Mufson, Wainer \& Levey 1983; Wolf, Eckenstein \& Butcher 1984) and may correspond to the ascending reticular activating system of Moruzzi and Magoun (1949). Jones (1985a) has postulated that the cholinergic cells in the LDTn and the PPTn may function as relay cells receiving input from various reticular nuclei. The cholinergic afferents to and the chemical identity of the cholinoceptive reticular neurons remains to be determined.

We note, however, that our extracellular recording method permits us to determine whether cells are segregated in ChAT fields. Precise localization can be achieved only using intracellular procedures. 
Recent single-unit studies conducted by Shiromani and McGinty (1986) do not support the view that certain regions (e.g., FTG) tend to favor excitatory feedforward activity. Shiromani and McGinty (1986) monitored the activity of medial PRF neurons following localized microinfusions of carbachol and found increased ( $28 \%$ of 56 neurons sampled) and decreased (55\%) patterns of medial PRF activity occurring in conjunction with a carbachol-induced REM sleep episode. Neurons that were decelerated by carbachol were active during physiological REM sleep, suggesting that a majority of medial PRF neurons may be nonspecifically activated during REM sleep and thus may not play an instigating or modulating role in REM sleep generation. The carbachol-induced neuronal deceleration could not be explained as a result of impairment of spike-generating mechanisms since normal firing levels were achieved during movement. This finding therefore reinforces the suggestion (Siegel 1979a) that a majority of medial PRF neurons subserve motor function.

Whereas a majority of medial PRF neurons were decelerated, carbachol activated a subpopulation (28\%) of medial PRF neurons, with the increased activity either preceding or occurring simultaneously with the development of the tonic and phasic components of REM sleep. Moreover, these neurons displayed a significantly higher discharge rate during carbachol REM sleep than during physiological REM sleep. This finding therefore suggests that activation of some medial PRF neurons may produce a reticular state highly conducive to REM generation. The cells whose discharge rates were increased and decreased by carbachol were not localized to a particular medial PRF region, thus supporting the view that a diffusely represented PRF neuronal network may generate REM sleep (McGinty 1985). Recently, Green and Carpenter (1985) found that iontophoretic administration of acetylcholine elicited both incremental and decremental responses in the para-abducens reticular formation, a region where carbachol readily evokes a REM-like state. Other studies have reported similar findings (Bradley, Dhawan \& Wolstencroft 1966; Green \& Carpenter 1981; Olpe, Jones \& Steinmann 1983).

In another study, Shiromani et al. (1986) monitored blood pressure (BP) following microinfusion of carbachol into midbrain, pontine, and medullary sites. We, like Baghdoyan, Rodrigo-Angulo, McCarley \& Hobson (1984), found that pontine carbachol infusion readily triggered REM sleep. The BP levels during carbachol-induced REM sleep were similar to $B P$ levels seen during normal REM sleep. However, carbachol infusions into midbrain and medullary sites produced arousal and a pronounced hypertensive effect. A similar cholinomimetic-induced hypertensive effect has been noted in anesthetized rats (Kubo \& Misu 1981a; 1981b) and cats (DeWildt \& Porsius 1981a; 1981b). We therefore believe that the suppression of REM sleep after midbrain or medullary carbachol infusions observed by Baghdoyan, Rodrigo-Angulo, McCarley, and Hobson (1984) may have been a result of severe cardiovascular abnormalities.

Even though much progress has been made in understanding the REM process, identification of the neuronal mechanism(s) responsible for REM sleep has proved to be difficult principally because the PRF contains diffusely represented networks of interconnecting cell groups. To help elucidate the role of different cell groups in REM sleep it will be necessary to use a combination of neuroanatomical and neurophysiological studies. Neuroanatomical studies might combine horseradish peroxidase and immunohistochemical techniques to characterize the synaptic connectivity of the medial PRF neurons. At the neurophysiological level it will be necessary to stimulate the medial PRF pharmacologically and to monitor neuronal discharge patterns. The proportion of excitatory and inhibitory neuronal responses temporally related to REM sleep will provide clues regarding the receptor mechanisms and interneuronal connectivity responsible for REM generation. It is important to deter- mine whether the PRF-induced excitation that is critical to REM generation is uniquely linked to cholinergic mechanisms. Might other excitatory agents activate PRF activity and promote REM sleep? We believe that a diffuse PRF excitation as produced by excitatory agents such as glutamic acid will not influence REM sleep. We suggest, instead, that a PRF activation mediated specifically by periodic activation of muscarinic receptors may be crucial for REM sleep generation.

\section{Location of the systems generating REM sleep: Lateral versus medial pons}

\author{
Jerome M. Siegel and Dennis J. McGinty \\ Sepulveda V. A. Medical Center, Sepulveda, Calif. 91343
}

Hobson et al. are to be congratulated for their acceptance of the evidence inconsistent with their original hypothesis. This evidence includes findings that:

(1) Neurons in the medial pontine reticular formation do not discharge selectively in REM sleep (McGinty, Harper \& Fairbanks 1974; Siegel \& McGinty 1977; Siegel, McGinty \& Breedlove 1977; Vertes 1977). Although intracellular recordings of these cell groups have produced new data, these do not bear on the the selectivity issue unless control recordings are made from other pontine cell groups and the entire profile of the waking-sleep cycle discharge is compared. The original error, which was based on the absence of movement in the headrestrained animal, should not be repeated in the intracellular series.

(2) The best site for cholinergic elicitation of REM sleep is not in the gigantocellular tegmental field (FTG) region of the medial pons.

(3) Total destruction of the medial reticular formation of the pons does not prevent REM sleep (Sastre, Sakai \& Jouvet 1981; Drucker-Colin, Bernal-Pedraza, Fernandez-Cancino \& Morrison 1983). What is most striking about these two studies is not that REM sleep eventually recovers from the lesions but rather that these gigantic lesions of the medial reticular formation are almost completely without effect on REM sleep. REM sleep was reported to be present in nearly normal amounts within 24 hours after the lesion (Sastre et al. 1981). Hobson et al. argue that there are many examples, such as suprachiasmatic nucleus (SCN) lesions, in which a total function is not lost after the lesion. However, although not all circadian rhythms are lost after SCN lesions, there is a clear discrete deficit, loss of some circadian rhythms, including that of sleep-wakefulness. Analogous arguments can be applied to the other lesions mentioned by Hobson et al., in contrast to the lack of effects of FTG lesions.

Having accepted these data, where do we go from here? The approach taken by Hobson et al. is to hypothesize a distributed system, organized so that destruction of any one of its elements does not disrupt REM sleep. This is a very attractive idea, since it is a truism that few functions are entirely localized to a small group of neurons. In fact, we have ourselves previously proposed theories of distributed generation of REM sleep (Siegel 1979b; McGinty 1985). However, the reciprocal-interaction model would seem to require the existence of selective REM-on neurons. Although a few localized REM-on neurons have been described, these have been implicated only in the atonia of REM, not the generation of REM as a whole. We fail to see the advantage of a distributed system of neurons that do not fit the model.

Nearly all models accept the evidence from pharmacological and unit-recording studies that putative aminergic REM-off neurons modulate REM and that the suppression of aminergic functions may permit transitory increases in REM. Hobson et al. now emphasize that a distributed system of aminergic neurons is an essential component of their reciprocal-interaction 
model. A distributed system is required because it is known that destruction of the largest noradrenergic nucleus, the locus coeruleus, does not block REM sleep (Jones, Harper \& Halaris 1977), whereas transections behind the dorsal raphe nucleus, the largest collection of serotoninergic neurons, do not alter the REM cycle in the pontine preparation (e.g., Sterman, McGinty \& Iwamura 1974). Thus, the model must be revised to speculate that some additional poorly studied neurons, not yet directly implicated in REM control, can serve the required aminergic function. In addition, it has been 10 years since the presentation of the Lotka-Volterra model of the reciprocal-interaction system. A major problem with this model is that it predicts that aminergic neurons will reach near-highest rates near the end of the REM period - as one would expect, since the theory states that they are excited by REM-on cells. All available data, including those of the Hobson group, fail to fulfill this prediction. The model must be revised.

Finally, recent evidence suggests that further localization of the REM-sleep-generating mechanism is possible. Jouvet first found that structures rostral to the pons were not required for REM sleep. Although REM sleep was present in brain stem structures after the transection, it was absent from forebrain structures. More recent studies utilizing transections between the pons and medulla (Siegel, Nienhuis \& Tomaszewski 1984) have demonstrated that pontine mechanisms are sufficient to generate REM sleep. Although REM sleep was present in the pons and forebrain of these preparations, it was entirely absent in the medulla and caudal regions of the neuraxis. The idea that REM sleep is generated by a distributed network would be strongly supported by evidence that neuronal activity in the forebrain or medulla of such preparation showed the defining signs of REM sleep. However, we could see no evidence of this. Whereas a small piece of the neuraxis containing the pons shows virtually all the local signs of REM sleep, the massive forebrain and medulla-spinal cord, when detached from the pons, show no signs of REM sleep - despite the fact that both these regions contain cells that go off in REM sleep and cells that show the characteristic medial reticular pattern of activation during waking movements and REM sleep. No one would say that the forebrain and medulla make no contribution to REM sleep (in fact, it has been hypothesized that the medulla has a critical role in modulating the REM sleep state (Siegel, Tomaszewski \& Nienhuis 1986)); however, it is clear that the pons contains the neurons critical for generating REM sleep.

Having localized the neurons critical to REM sleep in the pons, can we then conclude that the REM-sleep-generating function is distributed throughout the pons? Recent evidence clearly shows that further localization is possible. Massive lesions of the medial pons have little effect on REM sleep (Jones 1985b; Sastre et al. 1981), but relatively small lesions of the dorsal half of the lateral pontine reticular formation can totally and permanently abolish REM sleep throughout the brain. Cholinergic stimulation of the medial pontine reticular formation produces REM sleep only after relatively long latencies, but stimulation of the dorsolateral reticular formation, outside the FTG, produces REM sleep at relatively short latencies. A recent mapping study using very small volumes of carbachol concluded that "sites located in the zone below the level of the ventral margin of PAC [periaqueductal gray] and above the level of the dorsal margin of FTG were significantly more effective in eliciting M4 [the state of complete atonia] than sites in PAG or in the principal nucleus of LC $(p<0.01)$ or in FTG ( $p$ $<0.01$ ) (Katayama, DeWitt, Becker \& Hayes 1984). In fact, although there are some ChAT-positive neurons in the medial pons, the greatest concentration is in the same dorsolateral area whose stimulation induces REM sleep at short latency (Kimura, McGeer, Peng \& McGeer 1981). Recent work on the medial reticular formation has shown that cells with very specific motor correlates are intermixed throughout various subregions of the reticular formation (Siegel \& Tomaszewski 1983; Siegel, Toma- szewski \& R. L. Wheeler 1983). Thus, cells related to tongue movement are restricted to a relatively small pontine region, although within that region they are intermixed with cells relating to totally different movements. Might there be a similar localization of cells related only to the atonia, EEG desynchrony, or other aspects of REM sleep? Or are the cells that are active during the motor activations of both waking and REM sleep critical for REM sleep generation? Recent evidence indicates that there are in fact cells that are selectively active in REM sleep. These cells discharge during REM sleep but are completely inactive during waking movement (Sakai 1985a). The greatest concentration of such cells is in the lateral pons, the same region that stimulation and lesion studies point to. It seems to us that this concordance of stimulation, lesion, and recording evidence indicates that a further localization of systems critical for REM sleep has been achieved. The lateral pons is the brain region critical for REM sleep. Medial pontine regions, including the FTG, are not critical for REM sleep generation. Furthermore, it is clear that, as is the case for reticulomotor functions, the neurons whose interaction is critical for REM sleep constitute only a small percentage of the cells within the lateral pontine region.

As we mentioned above, the fact that certain regions are not required for $\mathrm{REM}$ sleep generation does not mean that they make no contribution to REM sleep control. In fact, it is likely that all brain regions make some contribution to REM sleep. However, it is of considerable heuristic value to try to distinguish as precisely as possible those neurons that play critical roles in the REM sleep process from those that play minor or modulatory roles in its control. We can abandon the localizationist approach only when the data will not support the further identification of brain stem areas or cell groups critical for REM sleep.

\section{State control: Changing tools and language}

\section{Steriade}

Laboratoire de Neurophysiologie, Faculté de Médecine, Université Laval, Québec, Quebec G1K 7P4, Canada

The hypothesis of sleep cycle oscillation based on out-of-phase discharge by two brain stem cell groups has raised considerable interest and has triggered hot debates since it was proposed a decade ago. Some data on which the concept was based and some postulates of the 1975 model have been refuted. The rejected aspects mainly concern the selectivity of pontine reticular discharge during REM sleep and the crucial role played by giant pontine neurons in the generation of REM sleep events. Still, the reciprocal-interaction model remains something of an institution in sleep physiology. The reason is that this model has given impetus to research and has provided a useful framework to organize empirical data. The criterion of tonic latency and its methodology have been used by other investigators to reveal brain stem cell populations that exhibit precursor signs related to various electrographic aspects of waking and sleep states. On the conceptual side, the proliferation of drawings supposed to provide an image of REM sleep generation indicates that a part of the scientific community (although it has challenged many tenets of the model) has adopted the essence and proceeded to play variations on the reciprocal-interaction theme.

The spectacular developments in morphology and electrophysiology during the past 10 years require us to consider radical changes in both experimental tools and theoretical concepts. It is with these modern achievements in mind that I shall comment on the sleep model.

1. The old and the new version. The first impression may be that a drastic change is being proposed. Instead of relying 
exclusively on putatively cholinergic pontine giant cells with bifurcating axons to trigger all major events of REM sleep, the revised model distributes the responsibilities for such a difficult task. Not satisfied to locate REM-on populations in many cell groups of the brain stem, Figure l extends them to almost the whole central nervous system (the "REM-on cell network is widespread ... at the cortical, brain stem, and spinal cord levels") since the REM process "could theoretically start from several places within the network" and "any localization of a putative REM sleep generator even to the brain stem could, theoretically, be an illusion. "This is indeed very different from what we have known since 1962, when Jouvet showed that a chronic cat with a prepontine transection exhibits transient episodes of muscle atonia, spiky waves in the pons, and horizontal saccades similar to those of REM sleep in a normal cat. However, there is every reason to believe that Jouvet's findings are still valid; hence there is an urgent need to reveal the intimate cellular mechanisms of the brain stem oscillator that generates the process rather than diluting the search by worrying about cortical, thalamic, and spinal cord effectors or by considering various factors that may trigger the oscillator. The greatest advantage of such a diffuse network is a sort of insurance policy against disturbing results in the future, but the model becomes so flexible that it cannot be proved wrong.

The second impression is that the change is really not so drastic. Even if the demiurgic giant pontine cells are now replaced by more encompassing REM-on elements, these "are still assumed to be cholinoceptive and cholinergic," and the curve in Figure 5C still depicts REM-on neurons at their lowest discharge level during wakefulness, as was the case in 1975. Now, since the rules of the game have changed (at the present time, the REM-on cell network includes cortical, thalamic, midbrain, and other components), the term "cholinergic" and the curve itself are incorrect. Corticofugal and thalamocortical neurons may use aspartate or glutamate, but not $\mathrm{ACh}$ as a transmitter agent. With the exception of a cholinergic cell group in the caudal part of the midbrain (Ch5), the transmitters of neurons in the more rostral midbrain reticular core might be peptides or other unknown agents but - unfortunately for previous assumptions made by others as well as myself - not ACh. It is needless to emphasize that all long-axoned cortical, thalamic, and midbrain neurons are as active in waking as they are in REM sleep. Similarly, unit recordings of antidromically identified neurons in the cholinergic pontine tegmental nucleus laterodorsalis projecting to the thalamus show a significant increase in firing rates during both waking and REM sleep, as compared to EEG-synchronized sleep (Sakai 1985a). These rate changes are similar to the increased cortical release of $\mathrm{ACh}$ in both waking and REM sleep states (Jasper \& Tessier 1971). Since the major source of cholinergic innervation of the cerebral cortex is the Ch4 group, I would predict that cortically projecting neurons of nucleus basalis will soon be shown to exhibit the same discharge profiles in wakefulness and REM sleep. The electrophysiological similarities between these two brainactivated states should not prevent us, of course, from searching for their still undisclosed differences at the cellular level.

In the light of the foregoing data, however, one is puzzled by some statements in the final section, such as "if the activity of cholinergic neurons is decreased, the result will be increased wakefulness" or "an increase in cholinergic neurotransmission . . . will favor the appearance of sleep." We have recent evidence that chemical or electrical stimulation of $\mathrm{Ch} 5$ or $\mathrm{Ch} 6$ cell groups in unanesthetized animals results in depolarization of thalamocortical neurons and blockade of rhythmic hyperpolarizing episodes, associated with EEG desynchronization, much the same as arousal does. What is needed is to attempt a new revision of the model depicted in Figure $5 \mathrm{C}$ and to admit that both cholinergic and aminergic neurons are at high levels of activity during waking. The striking difference between these two cell groups would occur only in REM sleep when aminergic neurons become silent, with obvious consequences for the cholinergic/aminergic ratio.

2. Structure and connections of chemically coded brain stem nuclel involved in state control. Because I feel it is in the model's interest to be confined within the limits of the brain stem, I shall not venture to comment on all the morphological issues raised by Figure 1 and Table IB. Rather, I will focus on some points that seem essential to the model: the reciprocal connections between locus coeruleus and raphe nuclei, on one hand, and the pontine reticular fields (so-called FTG, FTP, FTL), on the other; and the structure of the peribrachialis nucleus.

The assumption that locus coeruleus and raphe nuclei are reciprocally linked with the paramedian pontine reticular formation is not well documented. There is rather good evidence that the locus projects to the pontine reticular formation, although, since supporting data come from tracer injections into the crossroad of many fiber systems, there is still the possibility of uptake by passing fibers. There are much more reliable methods to study the retrograde labeling of neurons through selective uptake by aminergic axon terminals (see Cuenod, Bagnoli, Beaudet, Rustioni, Wiklund \& Streit 1982), and this must be done. When we come, however, to the reciprocal pathways (from the pontine reticular formation to the locus coeruleus) the situation is much worse, for we are left with data of the late 1950s, based on the whims of Golgi material and axonal degeneration following electrolytic lesions in the brain stem. Following horseradish peroxidase injections in to the locus coeruleus, "no HRP (labeled) neuron occurred in reticularis pontis oralis and caudalis, as well as in the nucleus gigantocellularis" (Sakai, Salvert, Touret \& Jouvet 1977, p. 27). Negative data should push us, of course, to try harder with more powerful techniques. Since only a few millimeters separate the locus coeruleus from the pontine reticular formation, intracellular staining with HRP may provide the required connections. I would have appreciated a greater emphasis on the lack of connections at the present status of knowledge, however, rather than some comfortable references.

The structure of nucleus peribrachialis is another key issue since most PGO-on bursting cells are located within its limits and these neurons may be involved in hallucinoid imagery during REM sleep, the stuff that dreaming is made of. If some neurons are called REM-on, they must obviously include peribrachialis ones. Yet, while in some parts of the target article peribrachialis neurons are regarded as cholinergic and as generating PGO waves, in some other parts and in Figures 1 and 2, neurons with the same nomenclature are considered aminergic, REM-off, and inhibitory. Only by looking at a small box and the legend of Figure $5 \mathrm{~B}$ will the reader realize that the peribrachial zone is a mixture of these two cellular types. Not only two: In addition to REM-off neurons and REM-on bursting cells related to PGO waves, there are many peribrachialis neurons with tonically increased firing and a high selectivity of discharge during REM sleep, about six times that in quiet waking or EEGsynchronized sleep (see Figures 5 and 6 in Saito, Sakai \& Jouvet 1977). If a nucleus has at least three types of neuronal discharges, is using at least two and probably three transmitters (a peptide is already there; see below), and is bombarding our brain during the night, we must rush into it with a modern arsenal in an adequate preparation (see section 4 below) to flesh out the "interpenetration gradient."

3. Transmitter actions. The model maintains that cholinergic REM-on cells are excitatory and aminergic REM-off cells are inhibitory. The point is that we can no longer talk exclusively in terms of classical PSPs with decreased input resistance. Moreover, the concept of the neuron has changed with the discovery of voltage-dependent ionic conductances and the implication that the same cell exhibits two opposite functional modes in two different states. And, surprisingly, in many respects $A C h$ and $\mathrm{NE}$ and 5-HT actions are similar. Let me enter into some of these details. 
In many cerebral structures, $\mathrm{ACh}$ as well as $\mathrm{NE}$ induces an increase in neuronal input resistance, blocks the calciumactivated potassium conductance, and blocks the accommodation of cell discharge. For $\mathrm{ACh}$, these effects have been obtained in the cerebral cortex in vivo (Krnjevic, Pumain \& Renaud 1971) and in the hippocampus in vitro (Cole \& Nicoll 1983). For NE, some actions similar to those of $\mathrm{ACh}$ have been observed in the hippocampus in vitro (Madison \& Nicoll 1982). The most frequent effect of NE on neocortical neurons in culture is enhanced synaptic activity, with increased frequency of both excitatory and inhibitory postsynaptic potentials (Rosenberg, Schweitzer \& Dichter 1985). It is no secret that NE hyperpolarizes the membrane of many neurons, but (like ACh) it raises their membrane resistance, thus leading to a striking increase in signal-to-noise ratio in many cerebral neurons (see Foote, Bloom \& Aston-Jones, 1983, for a review). The increased responsiveness of neurons that are targets of NE-ergic elements may explain, for instance, why pontine reticular neurons show clustered excitations triggered by inputs related or unrelated to movements during wakefulness, when NE-ergic cells are most active (see Figure 42B in Hobson \& Steriade 1986). One can argue that some of these actions, which depart from the postulated inhibitory nature of NE-ergic neurons, occur in the thalamus and cerebral cortex, far away from the brain stem sleep oscillator. In fact, troublesome results are also reported in the very center of REM sleep generators, where NE excites $85 \%$ of bulbar reticulospinal neurons (Hösli, Tebecis \& Schonwetter 1971) and pontine reticulospinal neurons show an approximately equal frequency of long-lasting excitatory responses and fast inhibitory responses to either $\mathrm{ACh}$ or 5-HT (Greene \& Carpenter 1985). Greene \& Carpenter conclude that both substances may produce exactly the same response; they suggest that the opposing actions of the same substance may be due to segregation of transmitter receptors over different portions of the somadendritic membrane. Such a suggestion can only be tested in a simplified preparation (see section 4 below).

To make matters even more complicated, peptides invade the scene in a way that disturbs our current views. Neuropeptide $Y$ is revealed in many cortically projecting NE-ergic neurons of the locus coeruleus (Gustafson \& Moore 1985), and somatostatin-immunoreactive cells are found in the medullary raphe system (Bowker \& Abbott 1985), not to mention the betterknown substance $P$ in the rostrally projecting laterodorsalis and peribrachialis cholinergic neurons (Vincent, Satoh, Armstrong \& Fibiger 1983). Whether this colocalization of peptides will prove to produce actions with the same or with opposite signs compared to those of the more conventional transmitter, is an open question. Both synergic and antagonistic effects (say, between peptides and $\mathrm{ACh}$ ) are reported (Lamour, Dutar \& Jobert 1983). But it is impossible to discuss further the mechanisms of enduring states, operating on long time scales, without calling upon these newly arrived and troubling agents.

4. What should be done? In addition to chronic experiments on behaving animals that are designed to answer questions at a global level but cannot reveal intimate cellular mechanisms, there are at least two types of acute experiments that must be undertaken. In both of them, the demonstration of a brain stem oscillator that may operate transiently for NREM-REM cycle in the absence of the forebrain must be considered, and, if any doubts are entertained about Jouvet's 1962 results, one must attempt to replicate them.

There would be no problem with the first type of experiment. Oshima and his colleagues analyzed arousal in motor cortical cells of a brain-stem-transected animal (see Oshima 1983, for a review); the same intracellular techniques can be used to look at neurons behind a high collicular transection. Unanswered questions, such as "excitation and/or disinhibition of these (pontine reticular) cells during REM sleep" may be investigated with the classical methods of measuring the rates of occurrence of unitary EPSPs and IPSPs as well as the increase or decrease in mem- brane impedance (see Inubushi, Kobayashi, Oshima \& Torii 1978). Even more exciting questions can be studied, such as, Does microstimulation of locus coeruleus or dorsal raphe nucleus indeed elicit short-latency (not to say monosynaptic) hyperpolarizations in pontine reticular cells? If so, are these hyperpolarizations associated with increased or decreased input resistance? These questions have been waiting for an answer since 1975. Of course, the results of such experiments are crucial as a test of the model.

The second type of experiment is even more exciting. It takes advantage of Llinás's isolated mammalian brain in vitro preparation (Llinás, Yarom \& Sugimori 1981). This technique, which utilizes the vascular system to irrigate portions of the brain, allows one to study the whole brain stem and cerebellum with preserved integrity of circuitry, and provides the possibility of investigating long-term changes in electrical or biochemical properties of the cell groups hypothesized to form the oscillator.

Many questions raised by the target article would then be answered. This must be done, since otherwise young people will leave the sleep field, as they left the ascending reticular concept for almost two decades, between the 1950s and the 1970 s, because they believed that brain stem affairs were not amenable to the morphological and electrophysiological techniques of the time. The concept of reciprocal interaction is too stimulating to allow it to fall into oblivion by not doing such analyses.

\section{Ascending cholinergic and serotonergic control of the electrocorticogram: Do I see a ghost?}

\section{H. Vanderwolf}

Department of Psychology, University of Western Ontario, London, Ontario N6A 5C2, Canada

As one of the authors of a recent BBS target article (Vanderwolf \& Robinson 1981) which, I thought, had demolished the classical arousal-ascending reticular activating system theory, I was mildly surprised to find the old theory surviving, apparently unscathed, in the paper by Hobson, Lydic, and Baghdoyan. Is the theory truly alive and well, or am I, perhaps, dealing with a ghost, a dead resemblance of a formerly vigorous set of ideas?

The theory that activation of the neocortex is controlled by a reticulothalamocortical pathway is no longer tenable. Excellent activation of the neocortex continues to occur ipsilateral to a kainic-acid-induced lesion of the thalamus which produces nearly total destruction of thalamic neurons but spares fibers of passage. On the other hand, destruction of the substantia innominata, which provides a cholinergic innervation of the neocortex, results in a loss of one form of neocortical activation (Stewart, MacFabe \& Vanderwolf 1984). These observations are consistent with a wealth of previous evidence that cholinergic neurons provide an activating input to the neocortex (Krnjevic 1974; Vanderwolf \& Robinson 1981). Cholinergic fibers to the neocortex originate in the basal forebrain but not in the thalamus (Divac 1975; Johnston, McKinney \& Coyle 1979; Lehmann, Nagy, Atmadja \& Fibiger 1980). Hence, the thalamus is unlikely to be of crucial importance in cholinergic activation of the neocortex. However, it may be worth pointing out that reticulothalamic activity may influence the cholinergic cells of the basal forebrain via the projections of the intralaminar nuclei to the striatum and thence, via striatopallidal fibers, to the ventral pallidum or substantia innominata (Heimer 1978). A direct input from the mesencephalic reticular formation to the cholinergic basal forebrain cells is also a possibility.

In addition, recent evidence indicates that ascending serotonergic fibers produce an atropine-resistant (i.e., noncholinergic) form of activation of the neocortex. Such activation 
can be abolished by either reserpine or p-chlorophenylalanine and can be restored in reserpinized animals. by the administration of 5-hydroxytryptophan or serotonergic agonists (Vanderwolf 1984; Vanderwolf \& Baker, in press). Behavioral evidence indicates that cholinergic and serotonergic activation of the neocortex plays a major role in the control of adaptive behavior. Thus, it would appear that serotonergic neurons are important in the control of waking behavior and do not act merely to inhibit REM sleep as Hobson et al., propose. The Hobson et al. hypothesis is, in fact, based on an inadequate review of the available data. Serotonin usually has an inhibitory effect on neurons in anesthetized animals, but in the unanesthetized brain (and also following some anesthetics) it often has excitatory effects (Johnson, Roberts \& Straughan 1969; Jones 1982; Roberts \& Straughan 1967). Thus, it is unlikely that it acts merely as an inhibitor of "REM-on cells." Furthermore, it is not adequate to classify serotonergic cells as "REM-off cells." Approximately one third of dorsal raphe units increase their activity in correlation with the occurrence of phasic muscular twitches during REM sleep (Trulson \& Jacobs 1981). This activity may be responsible for the phasic atropine-resistant activation that occurs in the neocortex at these times (Robinson, Kramis \& Vanderwolf 1977). Since the Hobson et al. theory of sleep and waking is incompatible with all these facts, it seems to me to be merely a phantom theory and not a viable account of brain function.

Apart from specific points, such as those mentioned above, I think that the approach taken by Hobson and his colleagues is fundamentally incorrect. It seems to be assumed that the regulation of sleep, and of REM sleep in particular, is a major function of much of the brain. An alternative view is that the principal function of the brain is the control of waking behavior. Sleep can then be thought of as prolonged immobility, a behavioral strategy that some animals use to reduce food requirements and the risk of predation. Since there are animals that display little or no sleep, it is unlikely that sleep is a fundamental physiological necessity.

In mammals and birds, natural selection appears to have favored mechanisms that produce high levels of sustained motor activity. The selective advantage of endothermy may be due to this (Bennett \& Ruben 1979). However, it may be difficult to construct a nervous system that generates a high level of spontaneous motor activity (behavior) in part of the 24-hour cycle and complete inactivity in another part of this cycle. As a result, REM sleep may have evolved as a means of allowing the brain to generate "behavior" (i.e., motor pattern generators are activated) while avoiding the undesirable consequences of actually moving around (Vanderwolf 1983). Thus, REM sleep can be thought of as "behavior" occurring in the absence of the usual environmental controls and constraints. If this is true, the central problem becomes one of working out the brain mechanisms of waking behavior. The study of REM sleep becomes a side issue - interesting, but not of primary importance.

\section{Reciprocal interaction in sleep cycle control: Description, yes; explanation, no}

\section{Paul A. M. van Dongen}

\section{Weezenhof 35-36, NL-6536 HB Nijmegen, The Netherlands}

The attempt of Hobson, Lydic, and Baghdoyan to integrate data from several disciplines into a general theory about sleep cycle control is much appreciated. The nervous system is an integrating system, so an integrated theory is necessary to understand it. To subject a clearly formulated, causal, and testable theory to a public discussion is the way science proceeds - together, of course, with new experimental data and procedures. I agree with Hobson et al. that administration of cholinergic agonists in parts of the brain stem is a rather reliable method to induce a
REM-sleep-like state (part IV), but I do not agree that this is a recent development (cf. Cordeau, Moreau, Beaulnes \& Laurin 1963; George, Haslett \& Jenden 1964, Baxter 1969; Van Dongen, Broekkamp \& Cools 1978). I have selected two aspects of the reciprocal-interaction model to comment on in detail because (1) these aspects are important for the reciprocalinteraction model and other neural models, (2) because I feel qualified to discuss these aspects, and (3) because I presume that other commentaries will discuss other aspects.

Neurons say more than yes or no. Until rather recently, "the important and universally accepted point: different presynaptic fibers can exert one of two fundamentally opposite effects on postsynaptic neurons, either facilitation of inhibition" (Patton 1965, p. 168) was universally accepted indeed. Now we know that "excitation" is a good word for the action of glutamate and of acetylcholine via nicotinic receptors. "Inhibition" applies to the action of GABA and glycine. The other neurotransmitters have as a rule other actions. In Hobson's model the actions of noradrenaline and serotonin are referred to simply as inhibitory. In my youthful recklessness, I made a premature attempt to review the actions of noradrenaline in the brain (Van Dongen 1981), but now much more data on these actions are known (e.g., Waterhouse, Moises \& Woodward 1981; Waterhouse, Moises, Yeh \& Woodward 1982; Aghajanian \& Rogawski 1983; Vandermaelen \& Aghajanian 1983; Cahusac \& Hill 1983; North \& Yoshimura 1984). Noradrenaline does not simply cause inhibition. The same applies to serotonin. Several different actions of serotonin have been found in vertebrates (e.g., Wood \& Mayer 1979; Vandermaelen \& Aghajanian 1980; Aghajanian \& Lakoski 1984; Hounsgaard \& Kiehn 1985; Parker, Gundersen \& Miledi 1985; Van Dongen, Grillner \& Hökfelt 1986). The actions of noradrenaline and serotonin (and most other neurotransmitters) are different from excitation and inhibition, and this has far-reaching implications for their interactions. The classical neuronal wiring diagrams consist of schematic neurons with plus or minus signs (or open or filled circles) at their terminals labeled "excitation" or "inhibition" (Figures 1 and 2 of Hobson's paper). Nowadays this is an unacceptable simplification (unless one is sure that the only neurotransmitters involved are glutamate, acetylcholine, GABA, and glycine).

Information capacity of neuronal systems. The second argument is more abstract (or, if you will, more philosophical). In the cases investigated, neuronal systems transport an actual amount of information which is about equal to a priori information capacity. A priori information capacity is directly coupled to the number of neurons (Kulikowski 1971). For example, the number of fibers in our optic nerve is what is needed for our visual acuity and color distinction (at the given temporal resultion). Individual neurons convey different messages. In the reciprocal-interaction model the assumption is made that neurons (e.g., in the locus coeruleus or the dorsal raphe nucleus) are the same; what mattered would then simply be the number of firing neurons. It is as if one wanted to make sense of telephone communications along a cable with 36,000 lines (the bilateral human locus coeruleus has 36,000 cells; Vijayashankar \& Brody 1979), and then studied only the number of active lines instead of trying to make sense of the individual conversations. Functional models for a neuronal system of thousands of neurons that assume the system has an information capacity of only one or a few neurons are, in my opinion, too crude, too simplistic.

General. I will try to summarize the reciprocal-interaction model with a few simple key sentences and expressing my reaction in brackets.

Description:

REM-on cells:

Some neurons in the brain stem have a higher firing rate during REM sleep [agreed].

Firing of some of these neurons causes elements of REM sleep [agreed].

The latter neurons are cholinoceptive [agreed]. 
Acetylcholine causes an increase in the firing rate of these neurons [agreed].

These neurons are cholinergic [uncertain]. REM-off cells:

The locus coeruleus in the brain stem contains noradrenergic neurons [agreed].

The dorsal raphe nucleus in the brain stem contains serotonergic neurons [agreed].

Neurons of both nuclei have a very low firing rate during REM sleep [agreed].

The neurons are cholinoceptive [agreed].

Acetylcholine causes an increase in the firing rate of these neurons [agreed].

Firing of these neurons causes cessation of REM sleep [might be correct, but firing of these neurons also has other effects, and cessation of REM sleep also has other causes].

Explanation:

REM-on and REM-off cells are mutually connected [agreed, but these connections are not massive, and other mutual connections are present].

The REM-off cells inhibit the REM-on cells [not agreed; see above].

The actual sleep state depends on the number of firing neurons in each group [not agreed; see above].

Mutual interactions between REM-on and REM-off cells can explain the transitions between sleep states [not agreed].

I agree with the identity of the REM-on and REM-off cells and their possible role in the transition between sleep states, but I do not agree that interactions between REM-on and REM-off cells explain the transitions between sleep states. Hobson et al. also mention problems in this respect: the reciprocal-interaction model could not explain the time constant and period length of the sleep cycle (part II. B). Therefore "it may be speculated that an intracellular, metabolic event (such as protein synthesis or transport) also contributes to the time constants." And in modern neuroscience, "an intracellular, metabolic event" is almost synonymous with "any unknown event." Let's be fair and admit that we do not really know the mechanism of transition between sleep states.

\section{A new role for FTG neurons?}

\section{Robert P. Vertes}

Division of Basic Medical Sciences, Mercer University School of Medicine, Macon, Ga. 31207

I commend Dr. Hobson and his colleagues on their reformulation of the reciprocal-interaction hypothesis to make it more consistent with recent developments in the field of sleep. I have some difficulties, however, with the present synthesis as best addressed by the following three questions/issues.

1. Since the FTG area is no longer considered to be the trigger for the generation of the REM state, what is its present role in REM sleep?

2. When "REM-on" cells are active during waking (with movements or behavior) do their characteristics differ from when they are active during REM?

3. Is there a role for slow wave sleep in the new model?

FTG neurons: A new role? As Hobson et al. makes clear, their initial formulation of the reciprocal-interaction hypothesis was based, to a large extent, on the assumption that pontine gigantocellular (or FTG) neurons discharge selectively in REM sleep. As acknowledged by the authors, it was subsequently shown by myself (Vertes 1977; 1979) and by Siegel and McGinty (Siegel, McGinty \& Breedlove 1977) in the freely moving rat and cat, respectively, that FTC cells are as active during waking with movements as during REM sleep. These findings, together with recent demonstrations that kainic-acid-induced FTG lesions fail to alter REM sleep (Sastre, Sakai \& Jouvet 1981; Drucker-Colin \& Bernal-Pedraza 1983), indicate that FTG cells do not serve to generate the state of REM. Although Hobson et al. readily accept this conclusion (to the extent that the F.TG has been removed from the schematic model of reciprocally interacting cell populations; Figure 5), it appears that they continue to press for a fundamental role for the FTG in REM sleep. If FTG cells are not involved in REM state control, then what is their precise function in REM sleep?

The new model argues for distributed systems that become sequentially activated to generate the major indices of REM and hence the overall state. I essentially agree with this basic scheme, having recently advanced a very similar hypothesis (Vertes 1984). Hobson et al. list the major events of REM as cortical EEG desynchronization, muscle atonia, pontogeniculooccipital (PGO) spikes, and rapid eye movements, and they cite evidence localizing the triggering zones for each event to specific regions of the brain stem - muscle atonia and PGO spikes to the dorsolateral pons, cortical desynchronization to the midbrain reticular formation ( $\mathrm{RF}$ ), and rapid eye movements to a dorsal region of the caudal pons adjacent to the abducens nucleus. Each of these sites, however, lies outside the FTG, supposedly leaving the FTG without a role in either the generation of REM-associated events or the REM state.

Although I have for some time vigorously argued that the FTG region is not involved in REM state control, I have nonetheless proposed that the FTG serves to generate an important tonically occurring event of REM - the hippocampal theta rhythm (Vertes $1980 ; 1981 ; 1982 ; 1984)$. It is somewhat surprising that Hobson et al. make no mention of the theta rhythm.

In an investigation of FTG unit activity in the freely moving rat (Vertes 1979) I reported that $68 \%$ of FTG cells discharged selectively during waking movements and REM sleep and designated them MOV-REM cells. No cells were found to discharge selectively in REM sleep. The MOV-REM cells were further characterized by the following: (1) they could be subdivided on the basis of their pattern of firing into tonically and phasically discharging MOV-REM cells; (2) for the most part the tonic MOV-REM cells were localized to the rostral pons (pontis oralis) and the phasic ones to the caudal pons-rostral medulla (pontis caudalis and nucleus gigantocellularis); and (3) the tonically discharging cells were highly correlated with the theta rhythm of waking and REM (for an in-depth discussion see Vertes 1979; 1982), while the phasic MOV-REM cells were associated with phasically occurring movements (of the eyes, face, head, and neck) during waking and REM sleep.

The close correlation between tonic MOV-REM cell discharge and the theta rhythm suggested that these FTG neurons were involved in the generation of theta. To examine this possibility, I (Vertes 1980; 1981) mapped the entire brain stem (from the lower medulla to the caudal diencephalon) with microstimulation and showed that theta was most effectively elicited with stimulation of the pontis oralis-FTG area (the site of the greatest concentration of the tonic MOV-REM cells). In recent unpublished work I have found that bilateral kainicacid(KA)-induced lesions of the anterior pons (FTG), but not those outside it, completely eliminate the theta of REM and waking. As in previous studies (Sastre et al. 1981; DruckerColin \& Bernal-Pedraza 1983), these KA lesions did not disrupt other events of REM or the REM state. In summary, I see no role for the FTG in the new theory of Hobson and colleagues. I propose that FTG cells or a subset of them generate theta during REM sleep.

"REM-on" cells? The new theory is predicated on two distinct, interacting populations of neurons (albeit distributed ones) "REM-on" and "REM-off" cells. As Hobson et al. indicate, it is well established that cells in several monoaminergic groups discharge at lowest rates during REM sleep - thus definitionally 
qualifying as "REM-off" cells. One wonders, however, whether these same neurons might not also exhibit long silent periods during waking and, if so, what their function is during the silence of waking. I have greater difficulty, though, with the term "REM-on cells." Whether intentionally or not, Hobson et al. convey the impression that there is a population of brain stem neurons active solely or selectively during REM sleep. With the possible exception of a few REM-selective cells in the magnocellular reticular nucleus of the caudal medulla described by Sakai et al. (1981) - cells that may be involved in the antonia of REM - I am unaware of any brain stem neurons that have passed strict criteria for REM selectivity. This being the case, the term "REM-on" is a misnomer and should be replaced by something that reflects a neuron's totality of activity. For instance, with regard to FTG cells the appropriate phrase might be "waking-movement REM-on" cells or "MOV-REM-on" cells or the like.

The issue runs deeper than semantics, however. If "REMon" cells are active in states other than REM sleep (which they are), then a two-population model of reciprocally interacting cell groups does not appear adequate to account for the "extra" REM activity of "REM-on" cells. For example, the present model would predict that "REM-on" (or MOV-REM-on) cells are inhibited when "REM-off" cells are excited. However, during certain conditions of waking both "REM-off" and "REMon" discharge simultaneously, that is, REM-off cells do not inhibit "MOV-REM-on" cells. It seems that numerous difficulties of this sort arise if the model is to include (as it should) the waking-movement discharge properties of "REM-on" neurons. In my review of brain stem substrates controlling the events of REM (Vertes 1984), the issue of REM selectivity proved less problematic. For, as I pointed out: (l) none of the events of REM is specific to that state; each represents analogous waking activity, and (2) each REM-related event and its counterpart in waking is controlled by the same brain stem cell group. In effect, cells discharging during REM would be expected also to exhibit waking activity.

What is the role of slow wave sleep? In the new model, or for that matter the old, I have never fully understood the function of slow wave sleep (SWS) - if in fact there is one. In the diagram of reciprocally interacting cell populations (Figure 5) SWS is characterized by submaximal discharge of both on and off neurons. Are these intermediate levels of firing relevant to either the induction or maintenance of SWS? Or is the reciprocalinteraction hypothesis only concerned with interactions between waking and REM sleep? Since REM alternates with SWS (and not waking) it would be important to know whether Hobson et al. propose any mutual interaction between SWS and REM.

Finally, as I mentioned in the opening, I applaud Hobson's continued refinement of his model for sleep cycle control. Although (as discussed) I have some concerns with the new version, there is no question that the sleep field has greatly benefited from his global approach and will continue to do so.

\section{Authors' Response}

\section{When is a reflex not a reflex? The riddle of behavioral-state control}

\author{
J. A. Hobson, R. Lydic, and H. A. Baghdoyan \\ Laboratory of Neurophysiology, Department of Psychiatry, Hanvard Medical \\ School, Boston, Mass. 02115
}

The Sherringtonian reflex paradigm has successfully guided neurophysiology for almost a century. It has become increasingly clear, however, that reflex action may be abolished or even reversed during behavioralstate changes initiated by - and reflected throughout the central nervous system (CNS). The state-dependent aspects of CNS function suggest that the reflex paradigm may not be adequate to explain either the neuronal substrates regulating behavioral states or the cellular and molecular mechanisms by which behavioral-state changes are mediated.

The tension that we have experienced in conceptualizing states and in modeling their control was so strongly amplified in the foregoing commentaries as to suggest the sort of crisis characteristic of what Kuhn (1962) identified as "preparadigmatic science." Kuhn used this phrase to describe new fields of research that are still developing their particular scientific paradigms. Rapid-eye-movement sleep was first described only 33 years ago (Aserinsky \& Kleitman 1953), and the cellular basis of sleep has been studied only since the early $1960 \mathrm{~s}$. We have thus organized our response to these commentaries according to our still dim vision of an emerging paradigm, that of central nervous system states. This new paradigm confronts many processes which are neither acknowledged nor explained by the reflex paradigm.

A comparison of the CNS-state paradigm with the reflex paradigm suggests significant differences between the two. At the level of neuronal activity comparisons, these differences include discharge spontaneity (vs. input dependence), simultaneity of activation (vs. sequentiality), parallel processing (vs. linearity), ubiquity (vs. locality), persistence (vs. evanescence), and endogenous periodicity (vs. stimulus rhythmicity).

We do not mean to deny the validity of the reflex paradigm. Reflexology is admirably suited to solving the problems of connectivity on which the CNS-state paradigm must still rely. The CNS-state paradigm has emerged from the quantitative analysis of the spontaneous activity of neuronal populations over time. There may be a paradigm shift impending that is analogous to the differences between taxonomic biology (with its emphasis on species and lineages) and modern ecology (with its emphasis on the dynamic interaction within and between populations).

\section{On being of two minds: Analytic and synthetic}

The division of the scientific world into integrators and reductionists seems to be an inevitable category of the critical mind at work. It appears to be as difficult for our commentators as it is for us to capture and retain the best of both worlds. In this collection of commentaries, the canon of synthetic comprehensiveness, with its inherent tendency to oversimplify at the global level (Henn; Vertes), is pitted against the criterion of exactitude, with its tendency to select single details for the evaluation of entire programs of research (Vanderwolf; Siegel \& McGinty; Shiromani \& Gillin; Fishbein \& Bright; Jones).

Our response to the 30 commentaries provided by our colleagues around the world is dedicated to the principle that a pluralistic conceptual approach is preferable to a dualistic "either-or" logical position. We recognize that both global, synthetic, and simplifying approaches and microscopic, analytic, and complexity-recognizing ap- 
proaches are essential for any discipline that aims to understand the cellular and molecular basis of behavioralstate control. Ideally, such complementary approaches should be superimposed and simultaneous. In practice, our limited intellects may have to settle for an oscillating approach to this complex problem. This implies the ability to switch back and forth from reductionistic empiricism to integrative model building.

The present rebuttal was constructed from our pointby-point review of the commentaries. Before discussing their specific points, we present our general conclusions:

1. The set of quantitative physiological criteria (Hobson 1974) for the neuronal-level evaluation of the brain center concept has become the benchmark standard, the key organizing principle in sleep neurophysiology. These criteria include (a) the positive or negative selectivity of neuronal discharge for the behavioral state in question; (b) tonic latency and pattern of cellular discharge; (c) phasic latency and pattern of cellular discharge; (d) discharge periodicity; and (e) reciprocal discharge profiles of REM-on and REM-off cells.

2. Many of the commentators have considered only one of the criteria (positive selectivity) without recognizing or discussing the additive coherence of the remaining criteria that comprise the reciprocal-interaction concept.

3. None of the 30 commentators challenges in any way the fact that the foregoing criteria form the basis for all extant conceptual models of sleep cycle control postulated by numerous laboratories (target article, Table 2 and Figure 3). Nor did any commentator question the validity or the interpretation of the more than 40 experiments (Table 3 ) that constitute tests of the reciprocalinteraction model.

4. Most telling of all is the fact that even those who outspokenly call for scrapping the reciprocal-interaction hypothesis (Fishbein \& Bright; Jones; Morgane; Shiromani \& Gillin; Siegel \& McGinty) have offered no alternative explanatory model.

The absence of such specific refutations by the commentators emphatically reinforces our decision to selectively abandon or modify incorrect or outdated tenets of the reciprocal-interaction model until alternative integrative models appear. We thus avoid a nihilistic stance toward the important question: What are the cellular and molecular mechanisms underlying the ultradian sleep cycle?

The target article, the commentaries, and this response represent a dialectic approach to the neurobiology of behavioral-state control. Dialectics, by definition, is the process of confronting a thesis (the original tenet that the gigantocellular tegmental field [FTG] is a highly localized and discrete REM sleep generator) by an antithesis (the FTG is neither necessary nor sufficient for REM sleep generation). For us, the synthesis of this dialectic represents an effort to correct and revise the reciprocal-interaction model in accord with the wealth of new data that have accrued since 1975. Our commentators are sharply divided in their estimates of our success, but most of the commentaries contribute positively to the dialectical process.

All of the recent data indicate that any new models of sleep cycle control that might be proposed today will have to account for the reciprocal-activity profiles of the REMon and REM-off cells. All such models must also recog- nize the structural implications of the well-documented distribution, multiplicity, and interpenetration of cholinergic and aminergic neuronal populations.

In an effort to avoid a reiteration of points and positions already well defined in our target article, and to document the major conclusions stated above, we have searched the commentaries for data and arguments bearing on the following sets of questions:

1. Behavioral. What are the behavioral states whose neural control we are attempting to understand?

2. Structural. How do we identify which neurons are involved in behavioral-state control?

3. Dynamic. By what physiological mechanisms do such neurons regulate behavioral state?

4. Formal. What mathematical statements most concisely and adequately express the dynamics of these cellular mechanisms of state control?

5. New Approaches. What new concepts, techniques, and strategies for further experiments emerged from our target article and the commentaries?

\section{On thinking about behavioral states and their neural control}

We wish to clarify our definitions, goals, and strategies by way of answering, en masse, the host of "Why didn't you mention this?" questions by our commentators.

A state may be operationally defined as all the values of all the variables of a system at any instant in time (Ashby 1960). Because it is impossible to know all the values of all the variables of any biological system, we have focused our attention on certain states (e.g. , REM sleep), certain variables (e.g., neuronal firing), and certain values of those variables (e.g., rate, pattern, time course). Our selection of states, variables, and values is in turn guided by our concepts about the locus and mechanisms of control, as indicated in the following examples.

We have focused on the REM sleep state because it is easily identified (i.e., as a unique constellation of muscular atonia, EEG desynchronization, PGO waves, muscle twitches, and rapid eye movements); its control by the brain is clearly regional (pontine); and it is periodically recurrent (controlled by an oscillator).

Among the many variables available for the study of states, we selected neuronal action potential generation because the neuron is the structural unit of the nervous system and the action potential is a functional unit of the neuron. We have also sought to establish the connectivities and chemical responsiveness of the cells under study by means of neuropharmacological and neurophysiological identification procedures.

We selected neuronal discharge rate, pattern, and time course as measures because they can be quantified by a variety of techniques. In keeping with the emerging picture of the continuity of change in these variables that parallels the sequences of states, we have added the technique of time-normalized sleep cycle averaging to the more standard cross-sectional measures. Cycle averaging yields discharge rate profiles for single neurons, which can then be pooled to give a statistical representation of the phase-dependent activity of neuronal populations. 
The rationale for these choices and the definition of the variables and measures have been extensively discussed (Steriade \& Hobson 1976). As recognized by many commentators (Morgane; Daan et al.; Borbély; Siegel \& McGinty; Sakai), the approach allowed us to go further in characterizing the brain stem neuronal regulation of states than has been possible for the regulation of other behaviors. The emergence of a specific and testable neuronal model of state control is a sign of progress in behavioral neurobiology, whether or not the model is totally correct.

Some commentators debated the adequacy of the evidence and the logic of our reasoning concerning support for the reciprocal-interaction model. We will concentrate on these issues in this rebuttal. Some commentators expressed the wish that the model would go further to account for other processes (e.g., sleep deprivation, circadian control), other systems (e.g., hippocampus, habenula, hypothalamus), and other variables (histamine, dopamine, and peptides). We remind our readers that our target article was not intended as a comprehensive review; a recent review of sleep neurophysiology is given in Hobson \& Steriade (1986).

The data and the emerging model do nonetheless make important statements about behavioral arousal and its relative place on the continuum of states. And the model considers behavioral arousal at the level of single cells and in terms of the ratio of neurotransmitters. But the target article does not specifically account for the mechanism of switching between arousal and states of sleep (Borbély; Daan et al.; Henn; Kilduff \& Guilleminault; Jasper; Vanderwolf because we do not yet know enough to do so. If the switch between wakefulness and sleep is modulated by a circadian oscillator, it probably involves the suprachiasmatic nuclei (SCN). The signal to the pontine oscillator could well be a peptide (Henriksen; Krnjević; Normanton) or a neurotransmitter/modulator like 5-HT (Normanton). More research is needed to answer this question, and we and many commentators have suggested the concepts and strategies that new studies might use.

Circadian-ultradian interactions. We would like also to defend our conceptual and experimental choices on strategic grounds. The neuronal approach we have taken seems to us to be more aptly applied to the study of the NREM/REM sleep cycle than to many other behavioral rhythms. For reasons detailed below, we prefer the brain stem to many other admittedly important brain regions implicated in the control of these rhythms (e.g., hypothalamus Kawamura; habenula, or pineal gland, Normanton).

There has been an interesting historical parallelism between the development of neurobiological concepts about the regulation of circadian $(\sim 24 \mathrm{hr})$ and ultradian (much less than $24 \mathrm{hr}$ ) rhythms. We acknowledge that the circadian system (Moore 1982), localized in the basomedial forebrain, can be anticipated to strongly influence pontine systems implicated in the regulation of the ultradian NREM/REM sleep cycle (Kilduff \& Guilleminault; Kawamura). The conceptual advances in the field of circadian research have been facilitated by hypothesizing a locus of control (Richter 1965; Moore \& Eichler 1972) and developing sophisticated and quantitative ap- proaches to temporal organization (Pittendrigh \& Daan 1976) in the tradition of von Holst.

The multiunit recordings in "hypothalamic islands" by Inouye and Kawamura (1979) suggest an important path for future circadian research that may combine cellular electrophysiology with the formal analysis of circadian rhythms. The importance of working with identified single neurons has been discussed in detail (Steriade 1978). To date, there have been no single-cell recordings from the SCN of behaving mammals across even one circadian cycle. SCN neurons are small (8-12 micron soma diameter), they are located at the base of the brain, and the synaptic relationships between the SCN and its multitude of putative effectors remain unclear.

Therefore, although we appreciate the important contributions and continuing advances of circadian research, we are choosing for the time being to focus on the pontine substrates believed to regulate the ultradian sleep cycle. These pontine cells are of medium (30-40 micron) to large $(70$ micron) somal diameter and their size and location permits recordings across many sleep cycles. REM-on and REM-off cells can be recorded as single units, which means they can be identified on the basis of discharge properties and synaptic connectivity. Finally, since individual pontine neurons can be recorded across multiple sleep cycles, this provides an important opportunity to be quantitative, both in a statistical sense and with respect to the issue of temporal organization.

The selective inventory of cellular phenomena in REM sleep (target article, Figure 1, Table 1) prompts us to suggest that one of the most thoroughly studied mammalian behaviors at the cellular level is REM sleep. Considerably less is known about "sexual activity, speech, and memory processing" (Henriksen), and about that most heterogeneous of states - wakefulness (Vanderwolf). As Daan et al. have suggested, no behavior has been so thoroughly analyzed at the cellular level as sleep. We concur with Borbély that "a picture begins to emerge" and summarize in this rebuttal the picture of sleep neurophysiology as it is presently envisioned.

Other states and other variables. Other REM sleep variables, such as hippocampal theta, were also omitted, as noted by Vertes, who hypothesized that the FTG may generate the theta rhythm, as well as the eye movements and muscle twitches. We might also note that neither did we discuss state-related changes in heart rate, blood pressure, respiration, genital blood flow, or temperature control. Since behavioral states are, by the above definition, global, all physiological systems are involved in behavioral-state generation and maintenance. Some control element, which we assume to be neural, must temporally organize all such physiological changes, as Henn emphasizes. In order to have any chance of understanding some of the implicated control elements, our neuronally focused perspective has of necessity neglected other physiological systems. Such omissions do not imply that we think thermoregulatory or neuroendocrine systems play no role in regulating the sleep cycle.

What is the cellular basis of NREM sleep (Vertes; Borbély; Daan et al.; Kilduff \& Guilleminault)? Most of sleep is NREM sleep, and if sleep has any function at all (Henriksen; Vanderwolf), it must be served at least in part by NREM sleep. 
The oscillation between NREM and REM sleep is postulated to be timed by pontine neurons, operating via reciprocal interaction. This ultradian sleep cycle may in turn be timed by the SCN (as in the models of Daan et al. and of Borbély). In temporal, dynamic, and functional terms, NREM sleep is thus seen by the reciprocalinteraction model as an intermediate state between arousal and REM sleep. Likewise, at the cellular level the activity of REM-off and REM-on cell populations during NREM sleep are at intermediate discharge values. We thus propose that a similar cellular mechanism might generate both NREM and REM sleep when the distributed REM-on and REM-off populations are activated to varying degrees.

We are pleased to note that the behavioral-state concept appeals to those commentators who study the notoriously state-dependent motor system (Henn; Mori \& Ohta). We too are impressed with the way in which the operating properties of all pyramidal and extrapyramidal motoneurons and all final common path motoneurons participate in behavioral-state changes. Again we must remember that states are, by definition, all the values of all variables of all the elements of a biological system. So the motor system is, by definition, involved in behavioral-state control.

A related point regarding the causal analysis of behavioral states: Because of the system-independent aspects of behavioral state changes, it is sometimes (as with the FTG cells) impossible to distinguish between a neuron's role as controller and its role as regulated variable. One effect of a state change (e.g., PT cell burst discharge) can also be a cause of a state feature (e.g., a myoclonic twitch of an otherwise atonic muscle. The distributed generator concept (target article, Figures 1 and 5), with its recognition of feedforward and feedback excitation within and outside the confines of the brain stem, opens the brain stem to corticofugal (Greenberg) and other influences.

In the fifth paragraph of Part II of the target article we stated, "The excitatory interconnections are such that the REM-on activation process could theoretically start from several places within the network if the inhibitory aminergic input were removed uniformly." The following examples illustrate the meaning of this statement. It is known that even without removing the inhibitory input that exists during wakefulness, PGO waves can be reliably triggered and REM sleep can be precipitated by electrical stimulation of the lateral geniculate body (Nelson \& Hobson, unpublished observations). In the pontine cat, pinching the paw or tail produces what Jouvet called "reflex paradoxical sleep"; and DruckerColin et al. (1983) increased PGO wave frequency and reduced REM sleep latency with auditory stimuli. It might be argued that all roads (pathways) lead to Rome (the pontine reticular formation). That is just our point; as disinhibition moves the distributed generator network closer to the neuronal self-excitation characteristic of REM sleep, a phasic excitatory input arising from any one of many sources may be enough to initiate REM sleep.

What are the functions of behavioral states? It is assumed by Vanderwolf that natural selection has favored the development of behavioral states characterized by high degrees of outward motility - namely, waking. We are suggesting that immobility and even the internally gener- ated but constrained activation of motor systems might also be adaptive. For example, it might well be adaptive to limit motility to the most favorable diurnal phase: the light phase for sighted animals and the dark phase for the less visually acute but olfactorily gifted species. Such a function would be served by both the NREM and the REM phases of sleep.

Another functional concept related to the adaptiveness of sleep suggests that while the organism is sleeping (and thus both conserving energy and reducing the risk of predation) certain neuronal commodities essential to efficient waking-state activity could be restored. Such a function might be served by both NREM and REM sleep, but REM sleep may be the more efficient of the two.

For example, if one assumes that there is a positive correlation between aminergic neurotransmitter release (in the cortex) and aminergic neuronal firing rate (in the brain stem) it is reasonable to speculate that sleep may function to influence the turnover of serotonin and norepinephrine. Assuming further that the correlation between neuronal firing rate and neurotransmitter release is both quantitative and proportional, the sleep-related "savings" of norepinephrine and serotonin might be quite appreciable. This is because aminergic neurons show, on the average, a $50 \%$ reduction in firing rate in NREM sleep (Daan et al.) and greater than 90\% reduction in REM sleep. This hypothesis is intuitively appealing for several reasons:

1. The firing rate decrement of aminergic neurons over the sleep cycle constitutes prima facie evidence for the long-sought neural substrate of sleep as rest and restoration.

2. The aminergic system may be physically vulnerable to fatigue because, being comprised of medium-sized cell bodies and extensively branching axons, it has a large surface-to-volume ratio. Furthermore, because it discharges constantly throughout the waking period (Vertes), it may be subject to progressive depletion of transmitter as a function of time spent awake.

3 . One presumed beneficiary of the increase in synaptic efficacy that might accure from rest is the forebrain. The resumption of monoaminergic discharge and corresponding synaptic efficacy specifically matches the subjective improvement in human cognitive capacity after sleep.

At the same time that some neurons exhibit a REM-off discharge pattern, other neurons could discharge and facilitate synapse formation (e.g., during development), synaptic enhancement (postulated as a basis for learning), or synaptic maintenance (as homeostasis in mature animals). According to the reciprocal-interaction hypothesis, these functions may in part be mediated by changes in the aminergic to cholinergic neurotransmitter ratio across the sleep-waking cycle, an idea that appeals to Foote and to Henn.

Rather than viewing REM sleep as an evolutionary mistake or at best a pale copy of the real thing (wakefulness), we therefore place waking and REM sleep at opposite ends of a genetically assured continuum of behavioral states that have mutually enhancing functions. For example, it is known that NREM sleep latency and the percentage of NREM stage IV are both dynamically responsive to the duration and intensity of exercise in the preceding waking period. There is thus a functional 
interaction between states (Borbély; Daan et al.). We assume that this kind of interaction must be favored by natural selection. But since REM sleep is specifically a feature of the corticate mammals it would also seem to serve the so-called higher cognitive functions of the brain, such as learning and memory (Antrobus; Greenberg).

We share the Aristotelian bent of those commentators who feel that the understanding of mechanism is incomplete until the question of function is answered (Daan et al.; Henn; Henriksen; Krnjevic). We further share (with Daan et al.) the dialectical assumption that understanding functions may elucidate mechanisms and that understanding mechanisms may reveal functions. A key example is our conviction that something must rest (i.e., be restored) in sleep. Our muscles relax in sleep. Does that not imply conservation of acetylcholine in alpha motoneurons? Aminergic neurons decrease firing in REM sleep. Does that not imply conservation of neuromodulators at central synapses? We aim to be as pluralistic with respect to functional hypotheses as we are with respect to mechanistic ones. And we would be as surprised to find that sleep had only one function as we are loath to believe it has none (Vanderwolf).

What are the limitations in the applicability of the reciprocal-interaction concept? Since the reciprocalinteraction model is, to the degree just specified, a threestate model (W, NREM, REM), it is natural to apply it to sleep-waking disorders. Mendelson finds our analysis of sleep disorders intriguing but imperfect. For example, the model accounts well for some clinical findings but not at all for others, although the relevance of sleep studies for understanding affective illness is widely appreciated (Gillin \& Borbely 1985). We admit that although the similarities between narcolepsy and depression are explained, we have not yet accounted for the differences. We have a hunch the explanation may come when the neurons and cellular mechanisms mediating wakingsleep transitions are discovered.

The evidence for "a dopamine-release problem in narcolepsy" (Kilduff \& Guilleminault) is interesting, but we find this hypothesis difficult to incorporate into our concepts for two reasons. One is that dopaminergic neurons appear to be unique in not showing state-related changes in discharge rate or pattern (as pointed out by Monti), and, as we have admitted, we cannot yet precisely model any of the multiple forebrain influences that may mediate the circadian control of the pontine sleep cycle oscillator.

We also admit that the sedative action of tricyclics baffles us; both enhancement of amine efficacy and anticholinergic effects should impede sleep according to the reciprocal-interaction model. We point out, however, that our model is derived from effects at the brain stem level. Systemic drug administration is an altogether different paradigm in which drugs act at many different sites. Thus, the fact that the effects of parenterally administered drugs are often predicted by our model (Haefely) is supportive of the model, and it is not surprising that results from some studies using peripheral routes of drug administration cannot be explained by the reciprocalinteraction model.

In our review of clinical implications we also left out the vast and important area of epilepsy. Elazar reminds us of the remarkable empirical similarity between the PGO waves of REM sleep and the spike-and-wave complexes of epilepsy. We share Elazar's idea that behavioral-state control in cerebral health and disease is effected by local and diffuse neuronal excitability control. The revised version of the reciprocal-interaction model addresses those very issues in its emphasis on distribution and interpenetration of reciprocally discharging neuronal populations.

We also recognize that the behavioral-state concept lends itself nicely to the brain-based approach of the psychophysiological study of dreams. Some commentators expressed disappointment that we did not say much about the mental state correlates of the brain during REM sleep (Greenberg; Antrobus). We thought our task of addressing the neurobiological basis of REM sleep generation was sufficiently arduous. A revision of the psychological counterpart of the reciprocal-interaction model, the activation-synthesis hypothesis of dreaming, is in progress. It will consider the whole continuum of behavioral and mental states as they occur normally and in pathological conditions.

\section{On the localization of the sleep cycle oscillator: Which neurons do what?}

An initial criterion for a state-generating neuronal population was selectivity (meaning a state-specific change in firing rate; Hobson 1974). Some commentaries suggest that there is uncertainty about how to define and interpret this criterion (Jones; Morgane; Sakai; Steriade; Siegel \& McGinty).

Selectivity is defined as a greater than 2:1 ratio of geometric mean discharge rates of any given neuronal population in any one state relative to the same rate parameter in any other state. For convenience we arbitrarily use the REM sleep discharge rate as a reference, for comparison with NREM sleep and with waking. We most emphatically do not define all REM-on cells as selective (Jones; Sakai; Steriade). In our effort to convey the distributed nature of the REM sleep generator network, our Figure 5 (target article) appears to have misled many commentators on this point.

As Freedman recommends (and against Elazar's proscriptions), we ourselves are still hoping to find a neuronal population with the property of selectivity that could also sustain the other demands required for it to be considered the necessary and sufficient source of REM sleep activation. But as many of our commentators agree (and none deny), there are a least two cellular populations that show REM-sleep-specific selectivity. These neurons are nowhere near each other; hence the sleep cycle oscillator itself is, by this very criterion, distributed. This is a key point that must be acknowledged, but such an acknowledgment in no way means the model is less testable, as recognized by Mendelson. Anatomical distribution does, however, limit the analytic methods that can be used to explore the complexity of sleep cycle generation.

We would like to preface the discussion of REM-on and REM-off cells in the dorsal pontine tegmentum by thanking Sakai for his suggestions regarding anatomical nomenclature. Sakai's nomenclature is derived from his 
own extensive neuroanatomical and neurophysiological studies, which have enriched our thinking about sleep cycle regulation. His comments regarding the need for precise anatomical localization of REM-on and REM-off cells are well taken. However, we retain Berman's (1968) nomenclature in this response, because we feel it is important for clarity in discussing the historical aspects of these concepts, and because Berman's stereotaxic reference and terminology are deeply embedded in the domain of neurobiology.

One group of tonically active REM-on cells is in the medullary RF; it has selectivity ratios of about 700:1. The other group of selective REM-on cells is about $5 \mathrm{~mm}$ distant and is located in the anterodorsal pons. This anterodorsal pontine region has been extensively studied by us and by Sakai (1985c) and has been found by both laboratories to be populated with strongly state-specific REM-off cells in addition to the PGO burst (REM-on) cells. These REM-on cells fire in bursts with a stateselective relationship to ipsilateral rapid eye movements and ipsilateral geniculate, high-amplitude PGO waves. Sakai has also found tonic REM-on cells in the adjacent zone which he calls LC alpha and peri-LC alpha. Thus, with respect to Freedman's question, both positively and negatively selective cells with tonic as well as phasic patterns of discharge are found to be anatomically interpenetrated, as we have suggested (or intermingled, as Jones prefers), in this zone. This "interpenetration" is perhaps a Freudian concept, as Jones suggests, in the sense that it implies dynamic interaction between adjacent cells with different chemical identities and reciprocal discharge profiles.

Steriade and Foote, in recognizing all these facts, exhort us to invade the anterodorsal pons with the powerful armamentarium of cellular neurobiology. In an interpenetrated region like the peribrachial pons, however, how do we know which neuronal population is aminergic and which cholinergic, or that either REM-on or REM-off cells have cholinergic or aminergic identity (Sakai, Jones)? We cannot be sure. But, as Steriade and Sakai point out, this is one of the few brain stem regions where both aminergic and cholinergic cells certainly do coexist. Thus, the interpenetration of REM-on and REM-off and of aminergic and cholinergic cells is a foregone conclusion, whichever state-related firing pattern is correlated with whichever chemical class. So we certainly share the interest evinced in the more lateral areas of the pons by many commentators (Jones; Siegel \& McGinty; Shiromani \& Gillin).

But suppose there is, as some have hinted (Siegel \& McGinty; Jones), still another population of cells in the lateral pontine reticular formation that has a greater positive selectivity than any other cell group yet studied? Would we then agree that this is the "true" generator population and renounce forever the FTG, the PGO burst cells, and the medullary atonia generators as mere follower neurons?

Although we will be among the first to express interest in such a lateral pontine neuronal population, we will not assume its uniqueness (there may still be others) or its autonomy (it may not act alone). Our curiosity regarding any such population would be expressed in specific ways not altogether different from the questions posed in 1974 . For example, we would want to know:
1. Does the lateral pontine population show an earlier phase lead of its activity profile than cells in the FTG (tonic latency criterion)? Do lateral pontine discharge profiles phase-lead the medullary atonia generators (for which activity profiles still remain to be determined)? If not, how can one be sure which of these three populations activates which other population? As Foote affirms, showing that a lesion eliminates REM sleep cannot be taken as evidence for a prime generator because the lesion need only disrupt a critical node in an interactive network in order to disrupt REM sleep.

2. Does the lateral pontine population have as specific a relationship as some FTG neurons have to the phasic events of REM sleep? Is the lateral pontine population phasically activated before the PGO burst cells, as is the FTG population (the phasic latency criterion)? And do lateral pontine cells show the same discharge pattern as the well-known excitatory burst neurons (driving eye movements in waking and in REM sleep) that are located in the FTG just rostroventral to the abducens nucleus (Shimazu 1983)? What is the firing relationship of the lateral pontine cells to $\mathrm{PGO}$ waves? Is this relationship as precise as that of the PGO burst cells (the phasic latency criterion with directional specificity)? Unless these criteria can be met, lateral pontine cells can only be assumed to act in concern with those multiple, likewise distributed subgenerators of REM sleep.

3. How is any new putative generator population activated? Does it have pacemaker properties, as do the locus coeruleus and dorsal raphe neurons? How are these neurons turned on and off so as to account for the yet to be demonstrated discharge selectivity? In the absence of pacemaker input, must there not be either another prime mover or some other inhibitory population causing the putative generator to become excitable by disinhibition? In any case, there must be reciprocal interaction as either a cause or an effect or, as we believe, as both cause and effect. This is a logical necessity that must be recognized, be it for neurons in the lateral pons or elsewhere.

The distributed generator idea, shared by Siegel \& McGinty and by Sakai, seems to have troubled many of our commentators, who suggested, as we anticipated, that we were creating an untestable theory. (Jones; Henriksen; Morgane; Steriade).

Untestability is not an issue, as demonstrated by Table 3 in the target article. We can summarize our commitment to what we call causal hypothesis testing as follows. If a given putative generator can be selectively destroyed without affecting REM sleep, that population is neither necessary nor sufficient for generating or maintaining the state. This appears to be the case with both the pontine FTG and the LC, and both may be discounted as exclusive or unique prime movers, acting alone or in concert with each other to generate the REM sleep state. We also predict that the same findings might emerge following lesions of other REM-on and REM-off populations if they were lesioned one at a time. But if all these populations were stimultaneously destroyed, with no effect on REM sleep, we would have to abandon the model. This test, though difficult, may not remain technically impossible.

With respect to the anatomical extent of the generator population, one needs to distinguish between putative behavioral-state generators and putative physiological- 
trait generators. Our Figure 5 does not suggest that there are selective REM-on cells everywhere. Obviously that would be so nonspecific a trait as to be useless in thinking about control systems (Sakai; Steriade). Figure 5B clearly shows the keys indicating REM-on and REM-off cells and their relationship to schematically represented portions of the neuraxis. We do not propose as generators, for example, nonselective REM-on cells found in the cortex. A careful reading of the text and comparison with Figure 2 will show that we still rigorously define REM-on and REM-off cells according to the original selectivity criterion and, as Elazar has recognized, that all such cells are in the brain stem tegmentum.

It might be supposed that by so defining selectivity we are trying to save the old idea of the FTG as prime mover. We explicitly deny that assumption. We are trying to say, though, that we still regard the FTG selectivity data as valid in terms of tonic latency. To date no data have questioned the tonic latency for FTG cells (Sakai). The movement-related discharge of FTG cells found by Siegel \& McGinty in animals without head restraint represents an additional fact about FTG neurons but does not explain the undisputed increases in firing rate observed in FTG cells during REM sleep. In fact, no data have questioned the selectivity of FTG cells in head-restrained animals. In other words, the excitability of the FTG does change prior to the onset of REM sleep, whether or not that excitability change is necessary or sufficient for REM sleep generation. And we insist on two further points:

1. The movement-related firing of the FTG in the waking state may be caused by entirely different membrane and ionic mechanisms from the clustered discharge of REM sleep (McCarley's 1978 concept of selectivity of modulation applies here).

2. The kainic acid results do not demonstrate that the FTG has nothing to do with REM sleep generation under physiological conditions. To support this caveat, we provide yet another example: the well-known shifts in epileptic foci that bedevil surgical attempts to excise electroencephalographically defined seizure generators. When well-localized foci are excised, a new focus often appears elsewhere (Elazar \& Hobson 1985).

The other side of the oscillator: The REM-off cells. Another important point that we wish to emphasize is that the negative selectivity of the REM-off cells is every bit as interesting as the positive selectivity of the REM-on cells. The commentaries show that there is now widespread agreement that negative selectivity is a truly statespecific attribute of the REM-off cells that are assumed to be aminergic (McGinty \& Harper 1976; Sakai).

Some commentators still do not seem to appreciate how a group of cells that turns off in REM sleep could be considered a generator. We, too, were once troubled by this concept, finding it to be like the problem of the chicken and the egg. It is difficult to say whether the inactivation of the REM-off cells or the activation of the $\mathrm{REM}$-on cells comes first in the sleep cycle. If a diffusely projecting, anatomically distributed, and neurochemically interpenetrated population of neurons is tonically inhibitory to its postsynaptic domain then a decrease in its output will disinhibit (effectively excite) that postsynaptic domain. If that distributed postsynaptic domain in some way regulates the REM sleep state or trait generators, these generator neurons will undergo a simultaneous increase in their excitability. This problem would not be solved by finding evidence for Sakai's alternative synaptic model of reciprocal inhibition. A reciprocal system is, by definition, reciprocal, meaning that the cycle does not start in one place or another. (The question of whether serotonin and norepinephrine are properly considered to be either neurotransmitters or inhibitory is discussed in Part III of this rebuttal.)

We were surprised that no commentator disputed the finding of negative selectivity in aminergic zones of the brain stem. Does this mean that, in contrast to the FTG controversy, there is widespread agreement concerning the REM-off cells? Among our commentators are some of the many investigators to have independently observed this property. McGinty and Harper (1972) were the first to report the dramatic arrest of dorsal raphe neuron firing that were assumed to be serotonergic (all the work that has followed, including our own, indicates that this assumption was correct). For REM-off cells of the dorsal raphe nucleus, there are no exceptions to their sleeprelated firing pattern (Vanderwolf), no studies suggest that these cells exhibit long silent periods during wakefulness (Vertes), and raphe neurons uniformly display a significant decrease in discharge in association with the sleep spindles of NREM sleep (Daan et al.). In response to the query by Daan et al. we point out that the REM-off discharge profiles of the DRN have been shown to parallel those of the LC (Lydic, McCarley \& Hobson 1982). Foote clinched the assumption, first made by Chu and Bloom (1974a; 1974b), that the REM-off cells of the locus coeruleus were noradrenergic, a point on which there is now widespread agreement.

Whether or not the REM-off cells have the precise chemical identity we have assigned to them in our model, there is no doubt now that they exist. That is, there are neuronal populations with state-specific negative selectivity that are distributed in at least three brain stem zones.

Since selectivity, be it positive or negative, is an accepted criterion for a generator function, we have reason to conclude that the REM-off cells are, by definition, a part of the REM sleep generator. It therefore follows that the REM-off side of the oscillator is also by definition distributed. In addition, it is clear in many species, and most noticeably the cat, that the REM-off cells are intermingled with other neurons, including REM-on cells (Sakai 1985c). So we have no choice but to conclude that the distributed generator is also interpenetrated.

The evidence indicates that the sleep cycle generator is much more complicated than was first imagined (Antrobus; Fishbein \& Bright; Henriksen; Kilduff \& Guilleminault; Sakai; Steriade; van Dongen). Recognition of complexity of the system is the surest sign that sleep neurophysiology is moving forward conceptually. All areas of regulatory neurophysiology must deal with newly discovered complexities such as colocalization of neurotransmitters, the nonquantal release of neurotransmitters (Sun \& Poo 1985), heteroneuronal uptake of transmitters by neurons other than the one from which the transmitter was released (Zigmond, Baldwin \& Bowers 1985), and evidence showing that neurotransmitter release can occur as a function of presynaptic regula- 
tion, independent of discharge by the releasing neuron (Romo, Cheramy, Godehev \& Glowinski 1986). In receiving criticism from those who seem, like us, to yearn for a simpler world, we are reminded of Sophocles' aphorism: "None love the messenger who brings bad news." When faced with the ever increasing complexity of facts concerning CNS function, organizing principles such as the reciprocal-interaction model become even more essential.

The missing link: What mechanism silences the REM-off cells?We remain as curious as Elazar and Foote about the mechanism that so emphatically silences the REM-off cells. And we agree that the model, as synaptically diagrammed, does not account for this REM-off process. We thus acknowledge that the mechanism underlying the cessation of REM-off cell firing is, indeed, a missing link in the present scheme. It would be a simple matter for the model to interpose an inhibitory interneuron between the excitatory input from the REM-on cells to the REMoff cells. In our discussion of pathophysiology we suggest that GABA-ergic inhibition is one controlling input which may be so interposed (Haefely; Mendelson; Normanton). REM-on cell excitation might also be converted to inhibition if the REM-on cells acted via presynaptic action on the strongly inhibitory recurrent collaterals of the aminergic neurons that are accepted and formally recognized by our model. This would fit well with the picture of intense interconnections within the neuropil of the brain stem core that Scheibel has been emphasizing since the model was first proposed in 1973 (Scheibel \& Scheibel 1973). It may well be that Golgi anatomy is whimsical, as Steriade suggests, but we have been impressed by its power in the hands of such functionally oriented neuroanatomists as Ramón y Cajal.

\section{Reciprocal interaction: An obligatory inference still seeking definitive proof}

This dialcctic has revealed agreement that there are at least three and possibly four brain stem zones with unequivocally state-selective REM-on cells and at least three brain stem zones with unequivocally state-selective REM-off cells (see also Sakai 1985c). All of the REM-off cell zones contain aminergic neurons, and all workers agree in identifying the REM-off cells found there as aminergic. One of the REM-on cell zones contains cholinergic neurons, and all workers agree in identifying them as REM-on cells. How is one to conceive of the relationship between these robust and amply confirmed findings?

Logically, we have two choices:

The synchronous but opposite activity profiles of the putatively cholinergic REM-on cells and aminergic REM-off cells are either (1) both caused by the periodic activation (or inactivation) of some as yet undiscovered neuronal population (or populations) or (2) causally interdependent - i.e., they directly cause each other. We have already acknowledged that we are open to the first possibility and that we are ever on the lookout for evidence of a third oscillator influencing both REM-on and REM-off cells. To date, no such evidence has appeared. We take the second possibility no less seriously.
Perhaps the analogy of perfectly synchronized clocks will help to make our position clear. In the face of clear evidence for simultaneity in the time course of the oppositely directed activity profiles of pontine REM-on and REM-off cells across the sleep cycle, should we take the dualistic position of Descartes (who assumed that mind and body were two perfectly synchronized, but independent, clocks) or should we adopt the monistic position (that we are examining two interdependent aspects of the same process)? It is more than mere intellectual modernism that prompts us to take the latter position. We use Occam's razor to cut away redundant hypotheses where one will do. In spite of the defects of the reciprocalinteraction model, a searching review of the 30 commentaries suggests that the reciprocal-interaction concepts account for more facts with fewer assumptions than any model yet proposed.

There are three kinds of synaptic questions bearing on the limitations of the model:

1. Anatomical. Do the hypothesized synaptic connections actually exist?

2. Neurophysiological. Are the hypothesized signs of the synaptic connections correct?

3. Neuropharmacological. By what chemical messages are the hypothesized synaptic interactions mediated?

Few commentators either contested or substantially added to the anatomical evidence summarized in our Table 1B. Steriade and Jones noted the lack of strong evidence supporting the existence of connections from the pontine RF to the LC. Jones also pointed out that the FTG contains only a minimal number of noradrenergic varicosities. Thus, the structural substrates for interaction exist, but these are certainly not the strong, direct, and exclusive pathways suggested by the 1975 synaptic schema. Remembering Scheibel's interesting point regarding the nature of the contacts in the neuropil of the brain stem core, the unanswered question is: Are multiple weak connections sufficient to mediate the proposed reciprocal interaction?

With regard to the hypothesized synaptic signs of the model and the putative neurotransmitters postulated to convey those synaptic messages, we now discuss some of the evidence concerning the postsynaptic effects of the monoamines and acetylcholine and our rationale for maintaining the synaptic signs of Figures $2 \mathrm{~A}, 5 \mathrm{~A}$, and $5 \mathrm{C}$.

There appears to be widespread agreement that the assumption of auto- and heteroexcitatory actions of REMon cells is justified. The autoinhibitory action of REM-off cells is likewise widely accepted. But doubt is expressed by many of our commentators that the action of the REMoff cells is only (or even at all) heteroinhibitory.

Considerable evidence, derived from a variety of preparations, questions the notion (see Figure 1, target article) that monoaminergic neurons are uniformly inhibitory. In many systems monoamines appear to be excitatory. For example, L-dopa, the precursor of dopamine and norepinephrine (NE), induced walking in spinal cats (Grillner 1975), and 5-hydroxytryptophan (5-HTP), the precursor of 5-HT, has been shown to induce locomotion in spinal rabbits (Viala \& Buser 1969). Monosynaptic reflexes at the lumbar spinal level were facilitated by stimulation of the locus coeruleus (Strahlendorf et al. 1980), and NE has recently been shown to exert a facili- 
tative modulation of the monosynaptic brain stem reflex mediating jaw closure (Morilak \& Jacobs 1985). At the level of the pons, the iontophoretic application of 5-HT produced a fast inhibition and a long-lasting excitation of cells in the FTG (Greene \& Carpenter 1985). Pontine omnipause neurons that regulate saccadic eye movements have been shown to be inhibited by 5-HTP (Baloh, Markham \& Furuya 1982). At the cortical level the iontophoresis of NE decreased the evoked response recorded from most visual cortical neurons (Videen, Daw \& Raider 1984).

Although we acknowledge data contradictory to the hypothesis of heteroinhibitory REM-off cells, the monoamine inhibition hypothesis is retained in Figure 1 for heuristic and other empirical reasons (Haefely). This hypothesis should be tested at each brain level illustrated in Figure 1, using intact, unanesthetized animals.

Concerning the neuropharmacological assumptions of the reciprocal-interaction model, a recent iontophoretic study by Greene and Carpenter (1985) tested identified reticulospinal neurons in the peri-abducens reticular formation of the cat (medial FTG) for their responses to various putative neurotransmitters, including the three of specific interest to the reciprocal-interaction model: acetylcholine, norepinephrine, and serotonin. With respect to rapid responses, all neurons were excited by glutamine and aspartate and all were inhibited by GABA, glycine, and norepinephrine.

The clear-cut inhibitory action of norepinephrine was seen with neither serotonin nor acetylcholine, which evoked three different response patterns, each in about a third of the cells: fast inhibition, slow and long-lasting excitation, and a biphasic sequence of fast inhibition followed by slow excitation. Greene and Carpenter attributed this differential responsiveness to "a spatial separation of excitatory and inhibitory receptors on different portions of the cell" (p. 520).

In terms of the synaptic signs of the reciprocal-interaction model and the chemical mediation of those signs, unequivocal support is given only to the assumption of norepinephrine's inhibitory action, which we represent as a minus sign in our schema. Yet many commentators were reluctant to accept our view of norepinephrine as being either a neurotransmitter or inhibitory.

It is also a fact that two-thirds of the FTG cells responded with slowly developing excitation in response to acetylcholine. According to Greene and Carpenter's own interpretation, all of the cells probably have this response capability. How is one to represent this result in a synaptic diagram? We ask this question because we note, for example, in Greene and Carpenter's Figure 6 that the inhibition lasts 5-10 seconds and the excitation 20-30 seconds. In other words, although these results force us to grant that the plus sign we have given to ACh/REM-on cells may be an incomplete and therefore inaccurate statement, with what should the plus sign be replaced? For example, to reflect the dynamic aspects of these effects, would one symbolize the synaptic sign as -+ ; -++ ; or -+++ ? Rather than leave this question unanswered, we will say that in view of the statistical properties of the population model, we have every reason to be as interested in the late, long-lasting excitatory effects of acetylcholine as in the immediate inhibitory ones. This is because one of the effects we are trying to explain is the slow progressive increase in excitation of the REM-on cell population that is regularly observed in NREM/REM transitions.

Can we use the Greene and Carpenter data to support our idea that serotonin acts as an inhibitory neurotransmitter to REM-on cells? Although there is an initial inhibitory response, there is also a late excitatory response very much like that seen with acetylcholine. But suppose that the sustained inhibitory action of $\mathrm{NE}$ and the combined inhibitory-excitatory action of 5-HT were simultaneously withdrawn? Might that not leave the FTG population more susceptible to the late and sustained excitatory actions of ACh? It seems possible.

Our point is this: The Greene and Carpenter results certainly cannot be cited as refuting the functional assumptions of the model. In the first place, the results come from anesthetized animals, and in the second place, as Greene and Carpenter themselves state: "Although complicated by the phenomenon of excitatory and inhibitory responses to the same transmitter, these results are compatible with the Hobson-McCarley model of generation of desynchronized sleep" (p. 520).

The problem of serotonin: What does it do? When major theories (or parts of theories) are overthrown by new discoveries, there may be residual data that are neither contradicted by the new data nor explained by the new theory. Such is the case with Jouvet's (1969) serotonin theory of sleep generation. On the basis of lesion studies and pharmacological experiments using systemic drug administration, Jouvet proposed that 5-HT was responsible for maintaining NREM sleep and initiating REM sleep. This hypothesis became untenable when it was discovered that all the putative 5-HT neurons of the brain stem that were recorded with microelectrodes decreased discharge rate in NREM sleep and that release of 5-HT in the forebrain declined in parallel with the serotonergic unit activity in the brain stem.

These data clearly indicated that the only state that might be actively enhanced by serotonin is waking. Thus we welcome Vanderwolfs reminder that serotonin may mediate cortical activation in that state. We also share Foote's view that the waking state enhancement of aminergic neurons may be mediated by improved signalto-noise ratios in response to sensory inputs; the neuromodulators may, as their name suggests, mediate changes in the mode of information processing by the cortex (Krnjević). We assume this is what Jasper means in calling our attention to the striking differences in cognitive capacity during waking (when we attend and remember) and REM sleep (when we dream and forget). Conversely, only by a passive, permissive, or disinhibitory mechanism could serotonin be involved in NREM or REM sleep determination. We therefore see no way to affirm a direct role for 5-HT in the cortical activation of REM. Here we again assume, with Vanderwolf and Steriade, that the cholinergic neurons of the forebrain mediate cortical activation.

Even if Jouvet's original 5-HT theory has indeed been revised (see Jouvet 1984), Jouvet's data remain unchallenged and unexplained: How is one now to account for the insomnia that follows raphe lesions? Or for the subsequent restoration of sleep by 5-HTP? Certainly not by invoking the reciprocal-interaction model. We agree 
with Mendelson on this point. As Borbély points out, Jouvet (1984) accounts for these findings in his revised serotonergic theory of sleep by proposing that during wakefulness 5-HT acts as a neurohormone that regulates the synthesis and/or release of a sleep factor. The sleep factor, in turn, would be secondarily responsible for NREM and REM sleep.

We suggest that raphe lesion-induced insomnia could be due as much to fiber destruction and to neuronal disconnection as to raphe cell body destruction. One test of this hypothesis, the relatively selective destruction of raphe cells by neurotoxins, has been performed (cf. Monti) and sleep, though perturbed, was not eliminated. Another test, performed by Cespuglio et al. (1981), has been to cool the raphe to temperatures that stop serotonergic cell activity. The result of such cooling was not waking but sleep! In fact, many animals immediately began to generate PGO waves and entered REM sleep with a short latency after the raphe cooling. PGO waves are also increased by parasagittal knife cuts that disconnect, but do not harm, the raphe (see Figure 3.2).

The sedative effects of 5-HTP on raphe lesion-induced insomnia, like the allegedly sedative effects of tryptophan, are unlikely to be mediated serotonergically, for two reasons. One argument against 5-HT mediation is that the serotonergic neurons which might normally convert precursors to 5-HT are, by definition, destroyed by the lesion. The other line of evidence concerns the fact that tryptophan sedation - if it is real - is much too rapid to be ascribed to enhanced synthesis of 5-HT in serotonergic cell bodies. The synaptic effects of such enhancement would not be expected to reach synaptic endings in the cortex (or even the hypothalamus) for several hours after a tryptophan load. And tryptophan sedation has proved to be both a weak effect (when it is found at all) and an altogether absent effect in many studies that failed to replicate the initial findings (see Hobson 1983b).

Evocation of REM sleep signs by microinjection of cholinergic agonists. It is gratifying to note that so many of our commentators share our interest in neuropharmacological approaches to behavioral-state control and that their results are so consonant with the assumptions of the model (Elazar; Fishbein \& Bright; Haefely; Shiromani \& Gillin). Before discussing details, let us underline the areas of consensus: a REM-sleep-like state can be generated by experimental activation of some pontine reticular neurons (Shiromani \& Gillin); the generation of this REM-sleep-like state is mediated by muscarinic and possibly nicotonic acetylcholine receptors (Shiromani \& Gillin; Fishbein \& Bright; Haefely). We also wish to point out that systemic administration of physostigmine to decerebrate cats produces a REM-like state in which REM-on cells are activated and REM-off cells are inactivated (Pompeiano \& Hoshino 1976).

Both Krnjevic and Morgane chide us for not adequately acknowledging Hernandez-Peón's methodological innovations with central administration of $\mathrm{ACh}$ and his concept of a distributed REM sleep generator or circuit. We happily acknowledge both contributions, which we have consistently cited in our research reports (Baghdoyan, Monaco, Rodrigo-Angulo, Assens, McCarley \& Hobson 1984; Baghdoyan, Rodrigo-Angulo,
McCarley \& Hobson 1984; Baghdoyan et al. 1986). At the same time, we insist that those pioneering experiments were crude, since they used ACh crystals, did not present dose-response data or photomicrographs of injection sites, used no controls, and did not quantify sleep parameters. Supplying these necessary details is what we mean by "coming of age" experimentally. Krnjević also questioned whether the microinjection technique has been used to demonstrate that $\mathrm{ACh}$ is "normally the principal transmitter of sleep-related signals." We have shown that microinjection of neostigmine, an acetylcholinesterase inhibitor, evoked a REM-sleep-like state in a dosedependent manner, and that this effect was blocked by coadministration of atropine (Baghdoyan, Monaco, Rodrigo-Angulo, Assens, McCarley \& Hobson 1984). These data suggest that endogenously released $\mathrm{ACh}$ in the pontine tegmentum can trigger and maintain a REMsleep-like state.

Foote questions our assertion that chemical microinjection is methodologically unique. We admit that it is not problem-free. We have spent five years perfecting the method to the point that it is, at the very least, a reliable tool for inducing a REM-sleep-like state. The localizing value of the microinjection technique is still uncertain (Haefely), but we believe that it will prove to have a resolving power in the range of 1-2 millimeters. Whether microinjection of cholinergic agonists uniformly excites, excites and inhibits, or inhibits is also unclear, and may be in part a function of concentration (Haefely); but the methods to answer these questions are available (Shiromani \& Gillin). There can be no doubt that cholinergic microinjection is an effective and widely used experimental tool for the study of sleep cycle generation.

\section{On the mathematical model: What is the most concise description of the process of reciprocal interaction?}

A mathematical model of NREM-REM sleep state alternation must describe the following aspects of neuronal activity:

1. The continuous and symmetrically opposite changes in activity level of two brain stem populations of neurons (the REM-on and the REM-off cells).

2. The cooperative and competitive aspects of the known connections within and between the two populations.

3. The periodicity of the process.

We were therefore interested in the Volterra-Lotka equations, which were originally designed to account for the periodic fluctuations in the population size of species engaged in prey-predator interactions (McCarley \& Hobson 1975b). The equations have the requisite quality of generating continuous, periodic solutions that vary as a function of reciprocal feedforward and feedback connectivities of opposite signs. Since the behavioral-state concept necessitates a shift in focus from pathways to populations, ecologically derived models are formally appropriate.

Now we will.consider some of the issues raised by our commentators regarding the way the neurobiological facts have been combined into a quantitative expression of the ongoing neuronal dynamics. We share with all 
model builders the goal of explaining the maximum number of facts with the minimum number of assumptions. We therefore appreciate the perceptive comments of Antrobus, Borbély, and Daan et al., who recognize the nature of our attempt and are sensitive to both its utility and its shortcomings.

What are the assets and liabilities of the Lotka-Volterra model? For the benefit of those who may be less clear about this aspect of our work, we focus our discussion on our understanding of what the Volterra-Lotka equations can and cannot be expected to do in modeling the physiological data. As a first approximation, the Volterra-Lotka equations were chosen (McCarley \& Hobson 1975b) because they dealt with the following formal properties inherent in what was viewed as the "ecology" of the REM-on and REM-off cells: There are two populations of neurons, and as one population grows in activity level, the other declines in activity level. Here "activity level" denotes both discharge intensity (excitatory or inhibitory output) and excitability (or inhibitability). These functions are analogous to the number of individuals in prey and predator populations in the field biology situation. Some of our commentators noted that the many possible mediators of such periodic fluctuations in excitability include receptor sensitivity of the cholinoceptive REMon cells (Shiromani \& Gillin; Fishbein \& Bright). It should be clear, however, that any possible changes in receptor sensitivity do not arise spontaneously, as these commentators might suppose, but occur in the context of neuronal interaction.

These interactive considerations also relate to the comments of Daan et al. regarding the connectivity strengths, the terms $a, b, c$, and $d$ of the Volterra-Lotka equations. For all of the reasons discussed in Part III, these terms in the equation are arbitrary; they are unknown physiologically and unlikely ever to be known with any precision. But they can be reasonably inferred, and the inferences can be checked both mathematically and experimentally. The adjustments we have made in our revisions of the physiological model in no way improve or impede the attempt to derive estimates of these terms, but revisions do change the inferences that one might make concerning connectivity strength.

Let us consider just two examples to illustrate these points. The term a (see McCarley \& Hobson 1975b) represents the strength of the collateral (or feedback) excitation of the excitatory REM-on population upon itself. This is a term for which there is strong, and easily understandable, physical evidence: Collateral connections have been emphasized by every student of the reticular formation from Cajal through Brodal and Walberg to Scheibel and Graybiel; the exponential rise in average discharge level of REM-on cells in late NREM sleep is the corresponding physiological manifestation of this collateralization. What the Volterra-Lotka equations model is the change in level of excitation of the REM-on population as an exponential function (i.e., the product) of the activity level in the REM-on population and the connectivity strength within that population. In other words, the more the cells fire, the more they are likely to fire.

But in assuming that the term $a$ is a constant, the Volterra-Lotka equations are not adequate, especially in view of the clearly predictable change in the excitatory drive on the brain stem REM-on cells that is a necessary consequence of the progressive activation of more and more populations of neurons throughout the brain. In other words, the distributed generator concept brings with it recognition that the exponential increases in the excitatory level of REM-on cells of the brain stem is a function of not only collateral excitatory feedback between local and selective REM-on cells, but also the excitatory feedback from nonspecific REM-on cells outside the brain stem. Daan et al. clearly appreciate the problem for the mathematical model of recognizing this physiological reality.

Other reasons for doubting that the physiological process represented by the connectivity term $a$ is realistic as a constant include the following considerations. Synaptic efficacy (which is another way of saying "connectivity strength") is itself subject to dynamic change via at least two related processes: presynaptic transmitter depletion and postsynaptic receptor desensitization. Both depletion and desensitization could be expected to operate, in a nonlinear manner, during late NREM and early REM sleep when the REM-on cell population is being driven to fire at peak levels. Even small decreases in synaptic efficacy (due either to fewer quanta of transmitter per packet released or to fewer packets released per depolarization) or decreases in receptor sensitivity might account for the observed late-REM phase decline in REM-on cell activity. Thus there is a multiplicity of factors that might be as powerful as the symmetrically postulated increases in the efficacy of the inhibitory inputs represented by the connectivity strength term $c$ in the Volterra-Lotka equations.

The problem of periodicity: What is the time constant of the oscillator? In the ecological model, the time constant of the phase lag and the period of the oscillation are both determined, in part, by the gestation periods of the species engaged in prey-predator interaction. This is because in the presence of prey, which provide an abundant food supply, the predator population will produce offspring in numbers that will continue to increase after the availability of prey has begun to decline. What are the analogs in the case of neuronal populations?

There appears to be little or no phase lag between the peak activity levels of the REM-on cell and the low point of activity in the REM-off cell populations. Within sleep at least, the decrease in the activity of the REM-off cells appears to be mirrored by an increase in the activity of the REM-on cells. This is most simply modeled by direct synaptic action, which occurs in the time range of milliseconds or seconds.

The period length of the sleep cycle, however, which is in the range of minutes, cannot be modeled by synaptic delay. The reciprocal-interaction model, as drawn synaptically, would oscillate in the millisecond range (as Siegel \& McGinty correctly assume). Zepelin and Rechtschaffen (1974) and Campbell and Tobler (1984) have noted that the period of the sleep cycle (90-100 min in humans, 25$30 \mathrm{~min}$ in cats) is a function of the size of the animal, and more specifically correlates with the size of the brain, as quantified by the cephalic index. Thus, the period length of the sleep cycle may be a function of brain size. Since the denominator of the cephalic index is the width of the 
brain stem, and since the brain stem contains the sleep cycle oscillator, we have reason to take special interest in this spatiotemporal correlation. The possible role of slow protein transport $(100 \mathrm{~mm} /$ day) in mediating this correlation has previously been discussed (Hobson 1983b). Considerations such as this might help us answer Antrobus's plea for a specific substrate for sleep cycle length.

The period length of the sleep cycle may also be influenced by receptor sensitivity: Up regulation and/or down regulation of receptors is a time-consuming process affecting the excitability of the postsynaptic membrane. For Fishbein \& Bright, and for Shiromani \& Gillin, this process is an alternative "model" for sleep cycle regulation. For us it is neither an alternative nor a model. Regulation of receptors is a process to be considered and measured. If receptor regulation is found to covary with the sleep cycle, then it should be modeled.

Two findings relevant to the issue of sleep cycle duration come from recent studies that combined forced locomotor activity with single-unit recording (as suggested by Daan et al.). These techniques have shown that (1) dorsal raphe discharge rate can be significantly altered as a function of sleep cycle period length and that (2) following forced locomotor activity, sleep recovery occurred primarily by increasing the number of REM sleep episodes and REM-off discharge profiles, and only slightly by increasing REM sleep period length (Lydic et al. 1986a; 1986b). These findings are consistent with the hypothesis that the REM-off cell discharge profiles regulate the period length of the ultradian sleep cycle.

We hope that this discussion conveys a sense of how we use the Volterra-Lotka model in its unaltered state. It is beyond the scope of this review to discuss the specific revisions of the mathematical model that have been undertaken by our colleagues, McCarley and Massaquoi (1985; 1986).

\section{New approaches}

We invited and received suggestions regarding new experimental and analytic approaches to what we perceive to be a new era of hypothesis testing for the neural regulation of sleep. Some commentators resisted this invitation, suggesting that sleep neurophysiology was still at a hunter-gatherer phase of development.

Homeostasis and experimental perturbations of the cycle. There is in Freedman's idea of examining the effects of sleep cycle interruption an attractive analogy to the phase-shift paradigm of circadian rhythm research. If the ultradian sleep cycle were to be interrupted at, say $25 \%$, $50 \%$, and $75 \%$ of completion, what would happen to the NREM-REM phase partition and to the (purportedly underlying) cellular oscillator? Would it reset? Would it change frequency or amplitude?

Freedman is also tempted, as are we, to perturb the NREM-REM phase transitions when the system is known to be unstable, as evidenced by "abortive" REM periods. We know that following forced activity the frequency of abortive REM sleep episodes decreases, and REM-off cell discharge profiles become more regular (Lydic et al. 1984; 1986a).

This phase-analytic approach might respond to what we perceive to be the spirit of the suggestion by Berbély and by Daan et al. that we concern ourselves with homeostatic aspects of sleep cycle regulation. We acknowledge that we have not yet studied the interaction of waking-state variables with either sleep cycle dynamics or responsiveness to cholinomimetics in our preparation.

Another of our commentators (Foote 1973) has used forced activity to study the dynamic response of cats to short-term deprivations of REM sleep; his evidence concerning sleep stages (like Lydic's data at the cellular level) confirms Borbély's suspicion that the sleep cycle is quantitatively sensitive to perturbations.

Now that we have the methods, the analytic techniques, and the conceptual models for cellular level analysis, we look forward to additional studies of the effects of systematic perturbations of the cycle.

Semiacute studies of decerebrate preparations. The program of research recommended by Steriade finds methodological and empirical resonance with our own emphasis on truly experimental interventions, for example, Baghdoyan's work using microinjection pharmacology. Elegant studies of postural muscle tone control (Mori \& Ohta), with the combination of microstimulation, spiketriggered averaging, intracellular recording, and HRP neuroanatomy, are further examples of experimental interventions applied to the question of sleep cycle control.

A research program using acute preparations might have as one of its goals the confirmation of Jouvet's localization of the REM sleep oscillator to the pontine tegmentum. It would also be interesting to determine whether a decerebrate preparation (such as that used by Mori \& Ohta) spontaneously evinces a periodic REM-like state with the same neuronal population correlates as found in intact cats. Another goal might then be to compare the spontaneous REM-sleep-like state of the pontine cat with the REM-sleep-like state that could be induced by carbachol in the decerebrate preparation. If those two states were similar, the cellular studies proposed by Steriade could be even more fruitfully pursued. Such a preparation might also facilitate direct neurophysiological tests of the connectivity assumptions of the model (Elazar).

Efferent copy: Exploring the mechanism and functional significance of the PGO wave system. The location of PGO burst cells in the path of the thalamically directed outflow of the cerebellum has attracted Antrobus's interest as well as our own. In early work of cerebellar unit activity it was noted that the Purkinje cells often fired in phasic anticipation of the eye movements of REM sleep, but unfortunately these studies did not look for directional specificity (Hobson \& McCarley 1972).

Now that we have a more detailed cellular picture of PGO wave generation and clear evidence for strong directional specificity (Cespuglio), a fresh approach to this possible substrate of efferent copy information is strongly indicated. Such an approach might incorporate the suggestions of Antrobus and of Henn that we establish the temporal relationship of the activity of PGO burst cells to saccadic eye movements in the waking state. Citing the post hoc fallacy, Henn correctly cautions that the determination of temporal sequence no more establishes cause in the control of state than it does in the 
command of movement. That is why phasic latency is only one of many criteria that we apply to our data.

In concluding this section we would like to underline the reciprocity between the state and reflex paradigms. Workers such as Steriade, Pompeiano, and Chase, take advantage of the knowledge and tools of sensorimotor physiology in detailing the circuitry that is differentially activated over the cycle of states. Mori \& Ohta take advantage of knowledge of states to investigate functional problems in sensorimotor integration. The REM sleep state also invites the interest of sensorimotor physiologists who aim to understand the autoactivation of motor pattern generators.

We especially welcome the words of the commentators who share our enthusiasm for the agenda of work we have jointly envisaged for the decade ahead.

\section{Conclusions}

We read the range of views expressed in the commentaries as representative of the two complementary intellectual positions, analytic and synthetic, that we defined to introduce this rebuttal. The commentaries clearly show that it is as important for the behavioral neurobiologist to be empirically and theoretically responsive to the global nature of behavioral states as it is to be uncompromisingly microscopic in testing specific cellular hypotheses about their mechanism and function. Where discrepancies exist between the demands of these two complementary mind frames, compromises in the form of patience, deeper thinking, and more persistent experimenting are preferable to the destructive outcomes of forced choice and premature closure.

This BBS Commentary has also demonstrated that there is no escaping the painful and arduous tasks of objectively and quantitatively defining behavioral states, making a strategically selective inventory of their cellular level correlates, and organizing those correlative data into a conceptual framework for testing emerging theories of sleep cycle generation experimentally. Although we have clearly not convinced all of our commentators that our own way of defining states, selecting neuronal correlates, and organizing neuronal data is the only way, or even a correct way, we trust that this dialogue has clearly demonstrated that:

1. The state concept is not only valuable but essential to behavioral neurobiology.

2. A conceptual shift from the old idea of neural centers to new concepts involving neuronal population dynamics is now necessitated by any comprehensive construal of the data.

3. The brain stem neuronal populations involved in state control are anatomically distributed and interpenetrated locally within the pons, and these pontine neurons interact with other populations at remote sites throughout the neuraxis.

4. The reciprocal-interaction model of NREM/REM sleep state alternation has conceptual and empirical strengths that compensate for its acknowledged imperfections.

We regard the evidence suggesting that the CNS is both simple (in its integrated aspect) and complex (in the details of its structure and function) as overwhelmingly convincing. In contemplating the deeper reasons for what might appear to be massive confusion surrounding this paradox, we wonder whether the field of behavioral neurobiology is in the throes of a paradigm shift. The neuronal-population approach is in its infancy, but we see it as indispensable to understanding the unified states of the brain. A research paradigm focused on CNS states cannot yet supplant or even satisfy the demands of the reflex paradigm. Under such conditions in the evolution of science it is normal to witness heated debate about the interpretation of data, obsessive concern with rules, and retreat to the shelter of the reigning (reflex) paradigm. The open peer commentary richly illustrates all three of these reactions, and others as well.

Let us, for emphasis, heighten the contrast between the two paradigms. Reflex systems operate in a serial linear manner; this operational characteristic is revealed and enhanced by certain experimental tests (e.g., stimulus-response) and is compatible with certain interventions (e.g., anesthesia). The linear systems constituting reflexes are themselves organized hierarchically so that fixed sequences of action and reaction are enhanced. Hierarchical aspects are likewise revealed and enhanced by the reflex paradigms used to study locomotion and respiration and by certain experimental interventions (e.g., electrical stimulation and transections).

But note: The rate and direction of information flow in the reflex systems and even their hierarchical ordering may dissolve, reverse, or otherwise change when measured across the domain of time in intact, unanesthetized animals. Many of the paradigms of reflexology are thus inappropriate to and incompatible with recognizing the inescapable reality of CNS and behavioral states. Furthermore, the analysis of time-dependent state regulation cannot afford to use only the reflex paradigm derived from the successful study of "sensorimotor" aspects of the CNS. Continued advances in behavioral neurobiology will require new paradigms specifically responsive to the neuronal-population dynamics of the CNS that are unique to behavioral-state control.

\section{ACKNOWLEDGMENT}

This work was supported by NIH grant MH-13923. We thank Chris Lindsey for typing.

\section{References}

Aaronson, S. T., Rashed, S., Biber, M. P. \& Hobson, J. A. (1982) Brain state and body position. Archices of General Psychiatry 39:330-35. [ta]AH]

Adrien, J. \& Lanfumey, L. (1984) Neuronal activity of the developing raphe dorsalis: Its relation with the state of vigilance. In: Experimental brain research. Supplement 8, Sleep mechanisms, ed. A. Borbély \& J. L. Valatx. Springer. [taJAH]

Aghajanian, G. K., Cedarbaum, J. M. \& Wang, R. Y. (1977) Evidence for norepinephrine-mediated collateral inhibition of locus coeruleus neurons. Brain Research 136:570-77. [taJAH]

Aghajanian, C. K. \& Lakoski, J. M. (1984) Hyperpolarization of serotonergic neurons by serotonin and LSD: Studies in brain slices showing increased potassium conductance. Brain Rescarch 305:181-85. [taJAH, PAMvD]

Aghajanian, G. K. \& Rogawski, M. A. (1983) The physiological role of $\alpha$ adrenoceptors in the CNS: New concepts from single-cell studies. Trends in Phannacological Sciences 4:315-18. [PAMvD]

Aghajanian, G. K. \& Wang, R. Y. (1977) Habenular and other midbrain raphe afferents demonstrated by a modified retrograde tracing technicjue. Brain Research 122:229-42. [taJAH, JRN] 
Akerstedt, T. (1984) Hormones and sleep. In: Experimental brain research, Supplement 8, Slccp mechanisms, ed. A. Borbély \& J. L. Valatx. Springer. [taJAH]

Albers, H. E., Lydic, R., Gander, P. H. \& Moore-Ede, M. C. (1984) Role of the suprachiasmatic nuclei in the circadian timing system of the squirrel monkey, 1. The generation of rhythmicity. Brain Rescarch 300:275-84. [ta]AH]

Amatruda, T. T., Black, D. A., McKenna, T. M., McCarley, R. W. \& Hobson, J. A. (1975) Sleep cycle control and cholinergic mechanisms: Differential effects of carbachol injections at pontine brain stem sites. Brain Research 98:501-15. [ta]AH]

Armstrong, D. M., Saper, C. B., Levey, A. I., Wainer, B. I. \& Terry, R. D. (1983) Distribution of cholinergic neurons in the rat brain: Demonstrated by the immunocytochemical localization of choline acetyltransferase. Journal of Comparative Ncurology 216:53-68. [PJS]

Aschoff. J. C. (1974) Reconsideration of oculomotor pathway. In: The neurosciences: Third study program, ed. F. O. Schmitt \& F. G. Worden MIT Press. [JA]

Aserinsky, E. (1969) The maximal capacity for sleep: Rapid eye movements density as an index of sleep satiety. Biological Psychiatry 1:14759. [SD]

Aserinsky, E. \& Kleitman, N. (1953) Regularly occurring periods of eye motility, and concomitant phenomena, during sleep. Science 118:27374. [rJAH]

Ashby, W. R. (1960) Design for a brain. Chapman and Hill. [rJAH]

Aston-Jones, G. \& Bloom, F. E. (1981) Activity of norepinephrine-containing locus coeruleus neurons in rats anticipates fluctuations in the sleepwaking cycle. Journal of Neuroscience 1:876-86. [SLF, ta]AH]

Azmitia, E. C. \& Segal, M. (1978) An autoradiographic analysis of the differential ascending projections of the dorsal and median raphe nuclei in the rat. Journal of Comparative Neurology 179:641-88. [taJAH]

Baghdoyan, H. A., Monaco, A. P., Rodrigo-Angulo, M. L., Assens, F., McCarley, R. W. \& Hobson, J. A. (1984) Microinjection of neostigmine into the pontine reticular formation of cats enhances desynchronized sleep signs. Journal of Pharmacology and Experimental Therapeutics 231:173-80. [taJAH]

Baghdoyan, H. A., Rodrigo-Angulo, M. L., McCarley, R. W. \& Hobson, J. A. (1982) Cholinergic induction of desynchronized sleep signs by carbachol microinjection shows intrapontine site differentiation. Slecp Research 11:51. [ta]AH]

(1984) Site-specific enhancement and suppression of desynchronized sleep signs following cholinergic stimulation of three brainstem regions. Brain Research 306:39-52. [taJAH, PJS]

(1986) A neuroanatomical gradient for the cholinoceptive induction of desynchronized sleep signs. Manuscript submitted. [tarJAH]

Baloh, R. W., Markham, C. H. \& Furuya, N. (1982) Inhibition of pontine omnipauser neurons in the cat by 5 -hydroxytryptophan. Experimental Ncurology 76:586-93. [rJAH]

Baraban, J. M. \& Aghajanian, G. K. (1980) Suppression of firing activity of 5 H'T neurons in the dorsal raphe by alpha-adrenoceptor antagonists. Ncuropharmacology 19:355-63. [taJAH]

Batsel, H. L. (1960) Electroencephalographic synchronization and desynchronization in the chronic "cerveau isole" of the dog. Elcctroencephalography and Clinical Neurophysiology 12:421-30. [HK]

(1964) Spontaneous desynchronization in the chronic cat "cerveau isolé." Archives ltalienncs de Biologie 102:547-66. [HK]

Baxter, B. L. (1969) Induction of both emotional behavior and a novel form of REM sleep by chemical stimulation applied to cat mesencephalon. Experimental Neurology 23:220-29. [PAMvD]

Beersma, D. G. M. (1984) Sleep structure in depression. In: Experimental brain research, Supplement 8 , Sleep mechanisms, ed. A. Borbély \& J. L. Valatx. Springer. [taJAH]

Beersma, D. C. M., Daan, S. \& Van den Hoofdakker, R. H. (1984) Distribution of REM latencies and other sleep phenomena in depression as explained by a single ultradian rhythm disturbance. Sleep 7:126-36. [ta]AH]

Bennett, A. F. \& Ruben, J. A. (1979) Endothermy and activity in vertebrates. Science 206:649-54. [CHV]

Berkinblit, M. B., Feldman, A. G. \& Fukson, O. I. (1986) Adaptability of innate motor patterns and motor control mechanisms. The Behavioral and Brain Sciences in press. [tajAH]

Berman, A. L. (1968) The brain stem of the cat. University of Wisconsin Press. [tar]AH]

Bizzi, E. (1966) Discharge patterns of single geniculate neurons during the rapid eye movements of sleep. Joumal of Neurophysiology 29:1087-95. [taJAH]

Bizzi, E. \& Brooks, D. C. (1963) Functional connections between pontine reticular formation and lateral geniculate nucleus during sleep. Archives Italiennes de Biologie 101:666-80. [ta]AH]

Boehme, R., Baker, T., Mefford, I., Barchas, J., Dement, W. C. \& Ciaranello, R. (1984) Narcolepsy: Cholinergic receptor changes in an animal model. Life Sciences 34:1825-28. [TSK]

Bonime, W. with Bonime, F. (1962) The clinical use of dreams. Basic Books. [RG]

Borbély, A. A. (1982) A two-process model of sleep. Human Neurobiology $1: 195-204 . \quad$ [AAB, taJAH]

Borbély, A. A., Baumann, F., Brandeis, D., Strauch, I. \& Lehmann, D. (1981) Sleep deprivation: Effect on sleep stages and EEG power density in man. Electroencephalography and Clinical Neurophysiology 51:48393. $[\mathrm{AAB}]$

Borbely, A. A. \& Neuhaus, H. U. (1979) Sleep deprivation: Effects on sleep and EEG in the rat. Journal of Comparative Physiology 133:7187. [SD]

Borbély, A. A., Tobler, I. \& Hanagasioglu, M. (1984) Effect of sleep deprivation on sleep and EEG power spectra in the rat. Behavioral Brain Research 14:171-82. [AAB]

Bowie, E. P. \& Herbert, D. C. (1976) Immunocytochemical evidence for the presence of arginine vasotocin in the rat pineal gland. Nature 261:66-67. [JRN]

Bowker, R. M. (1985) Variability in the characteristics of pontogeniculooccipital spikes during paradoxical sleep. Experimental Neurology 87:212-24. [ta]AH]

Bowker, R. \& Abbott, L. C. (1985) The nucleus raphe magnus: The relationship between the peptidergic and serotonergic neurons. Neuroscience Abstracts 11:124. [MS]

Bowker, R. M., Westlund, K. N., Sullivan, M. C., Wilber, J. F. \& Coulter, J. D. (1983) Descending serotonergic, peptidergic and cholinergic pathways from the raphe nuclei: A multiple transmitter complex. Brain Research 288:33-48. [taJAH]

Bradley, P. B. (1972) The actions of drugs on single neurones in the brain. Progress in Brain Research 36:185-86. [BE]]

Bradley, P. B., Dhawan, B. N. \& Wolstencroft, J. H. (1966) Pharmacological properties of cholinoceptive neurones in the medulla and pons of the cat. Journal of Physiology 183:658-74. [P]S]

Branchey, L., Branchey, M. \& Nadler, R. D. (1973) Effects of hormones on sleep patterns of male rats gonadectomized in adulthood and in the neonatal period. Physiology and Behavior 11:609-11. [WF]

Bremer, F. (1935) Cerveau isolé et physiologie du sommeil. Comptes Rendus de la Societé de Biologie (Paris) 118:1235-42. [ta]AH, HK, WBM]

Brodal, A. (1969) Neurological anatomy in relation to clinical medicine. Oxford University Press. [rJAH]

Brooks, D. C. \& Bizzi, E. (1963) Brain stem electrical activity during deep sleep. Archives Italiennes de Biologie 101:648-65. [taJAH]

Bucy, P. C., Ladpli, R. \& Erlich, A. (1966) Destruction of the pyramidal tract in the monkey. Journal of Neurosurgery 25:1-23. [taJAH]

Buttner-Ennever, J. A. \& Henn, V. (1976) An autoradiographic study of the pathways from the pontine reticular formation involved in liurizonlal eye movements. Brain Research 108:155-64. [ta]AH]

Cahusac, P. M. B. \& Hill, R. G. (1983) Alpha-2-adrenergic receptors on neurones in the region of the lateral reticular nucleus of the rat. Neuroscience Letters 42:279-84. [PAMvD]

Campbell, S. S. \& Tobler, I. (1984) Animal sleep: A review of sleep duration across phylogeny. Neuroscience and Biobehavioral Revicus 8:269-300. [ta]AH]

Campion, J., Latto, R. \& Smith, Y. M. (1983) Is blindsight an effect of scattered light, spared cortex, and near-threshold vision? The Bchavioral and Brain Sciences 6:423-86. [ta]AH]

Card, J. P., Brecha, N., Karten, H. J. \& Moore, R. Y. (1981) Immunocytochemical localization of vasoactive intestinal polypeptidecontaining cells and processes in the suprachiasmatic nucleus of the rat: Light and electron microscopic analysis. Journal of Neuroscience 1(11):1289-1303. [WF]

Cedarbaum, J. M. \& Aghajanian, G. K. (1977) Catecholamine receptors on locus coeruleus neurons: Pharmacological characterization. European Journal of Pharnacology 44:375-85. [ta]AH]

Cespuglio, R., Faradzi, H., Gomez, M. E. \& Jouvet M. (1981) Single unit recordings in the nuclei raphe dorsalis and magnus during sleep-waking cycle of semi-chronic prepared cats. Neuroscience Letters 24:135-38. [tarJAH]

Cespuglio, R., Gomez, M. E., Faradji, H. \& Jouvet, M. (1982) Alterations in the sleep-waking cycle induced by cooling of the locus coeruleus area. Electroencephalography and Clinical Neurophysiology 54:570-78. [ta]AH]

Cespuglio, R., Laurent, J. P. \& Calvo, J. M. (1976) Organisation anatomique 
des activités phasiques provoquées par la Réserpine au niveau du système oculo-moteur. Electroencephalography and Clinical Neurophysiology 40:12-24. [RC]

Cespuglio, R., Laurent, J. P. \& Jouvet, M. (1975) Etude des relations entre l'activité ponto-géniculo-occipitale (PGO) et la motricité oculaire chez le chat sous Réserpine. Brain Research 83:319-35. [RC]

Cespuglio, R., Verchere, G., Laurent, J. P. \& Jouvet, M. (1975) Correlations between central PGO activity and ocular movements in the cat. In: Sleep 1974, ed. P. Lewin \& W. P. Koella. Karger. [RC]

Chandler, S. H., Chase, M. H. \& Nakamura, Y. (1980) Intracellular analysis of synaptic mechanisms controlling trigeminal motoneuron activity during sleep and wakefulness. Journal of Neurophysiology 44:359-71. [ta]AH]

Chase, M. H., Enomoto, S., Nurakami, T., Nakamura, Y. \& Taire, M. (198I) Intracellular potential of medullary reticular neurons during sleep and wakefulness. Experimental Neurology 71:226-33. [taJAH]

Chase, M. H. \& Morales, F. R. (1983) Subthreshold excitatory activity and motoneuron discharge during REM periods of active sleep. Science 221:1195-98. [taJAH]

(1984) Supraspinal control of spinal cord motoneuron membrane potential during active sleep. In: Modulation of sensorimotor activity during alterations in behavioral states, ed. R. Bandler. Liss. [ta]AH]

Chu, N. S. \& Bloom, F. (1974a) Activity patterns of catecholamine-containing pontine neurons in the dorso-lateral tegmentum of unrestrained cats. Journal of Neurobiology 5:527-44. [taJAH]

(1974b) The catecholamine-containing neurons in the cat dorsolateral pontine tegmentum: Distribution of the cell bodies and some axonal projections. Brain Research 66:1-21. [ta]AH]

Coculescu, M., Serbănescu, Al. \& Temeli, E. (1979) Influence of arginine vasotocin administration on nocturnal sleep of human subjects. Revue Roumaine de Morphologie, d'Embryologie et de Physiologie 16:173-76. [JRN]

Cohen, M. I. (1979) Neurogenesis of respiratory rhythm in mammals. Physiological Reviews 59:1105-73. [taJAH]

Cole, A. E. \& Nicoll, R. A. (1983) Acetylcholine mediates a slow synaptic potential in hippocampal pyramidal cells. Science 221:1299-1301. [MS]

Conrad, L. C. A., Leonard, C. M. \& Pfaff, D. W. (1974) Connections of the median and dorsal raphe nuclei in the rat: An autoradiographic and degeneration study. Journal of Comparative Neurology 156:179-206 [ta]AH]

Cordeau, J. P., Moreau, A., Beaulnes, A. \& Laurin, C. (1963) EEG and behavioral changes following microinjections of acteylcholine and adrenaline in the brain stem of cats. Archives Italiennes de Biologie 101:30-47. [PAMvD]

Crowne, D. D. (1983) The frontal eye fields and attention. Psychological Bulletin 93:232-60. [JA]

Cuello, A. C. \& Sofroniew, M. V. (1984) The anatomy of the CNS cholinergic neurons. Trends in Neuroscience 7:74-78. [taJAH]

Cuénod, M., Bagnoli, P., Beaudet, A., Rustioni, A., Wiklund, L. \& Streit, P. (1982) Transmitter-specific retrograde labeling of neurons. In: Cytochemical methods in neuroanatomy, ed. V. Chan-Palay \& S. Palay. Liss. [MS]

Czeisler, C. A., Borbély, A. A., Hume, K. I., Kobayashi, T., Kronaure, R. E., Schulz, H., Weitzman, E. D., Zimmerman, J. C. \& Zulley, J. (1980) Glossary of standardized terminology for sleep biological rhythm research. Sleep 2:287-88. [taJAH]

Czeisler, C. A., Weitzman, E. D., Moore-Ede, M. C., Zimmerman, J. C. \& Knauer, R. S. (1980) Human sleep: Its duration and organization depend on its circadian phase. Science 210:1264-67. [taJAH]

Daan, S., Beersma, D. G. M. \& Borbély, A. A. (1984) The timing of human sleep: Recovery process gated by a circadian pacemaker. American Journal of Physiology 246:R16I-R178. [AAB]

Dahlstrom, A. \& Fuxe, K. (1965) Evidence for the existence of monoamine neurons in the central nervous system. Acta Physiologica Scandinavia 64 (Suppl. 247):1-36. [ta]AH]

Davis, H. T. (1962) Introduction to nonlinear differential and integral equations. Dover. [taJAH]

Delashaw, J. B., Foutz, A. S., Guilleminault \& Dement, W. C. (1979) Cholinergic mechanisms and cataplexy in dogs. Experimental Neurology 66:745-57. [taJAH]

Delorme, F. (1966) Monoamines et sommeils. Etude polygraphique neuropharmacologique et histochimique des états de sommeil chez le chat. Thèse Université de Lyon, Imprimerie LMD. [WBM]

DeWildt, D. J. \& Porsius, A. J. (1981a) Central cardiovascular effects of physostigmine in the cat: Possible cholinergic aspects of blood pressure regulation. Archives of International Pharmacodynamics 253:2239. [PJS]

(1981b) The influence of physostigmine on sympathetic outflow and haemodynamics by an action upon the pontomedullary region of the cat. Archives of International Pharmacodynamics 253:40-51. [PJS]

Divac, 1. (1975) Magnocellular nuclei of the basal forebrain project to the neocortex, brainstem and olfactory bulb: Review of some functional correlates. Brain Research 93:385-98. [CHV]

Drucker-Colin, R. \& Bernal-Pedraza, J. G. (1983) Kainic acid lesions of gigantocellular tegmental field (FTG) neurons do not abolish REM sleep. Brain Research 272:387-91. [ta]AH, WBM, JMS, PJS, RRV]

Drucker-Colin, R., Bernal-Pedraza, J., Fernandez-Cancino, F. \& Morrison, A. R. (1983) Increasing PGO spike density by auditory stimulation increases the duration and decreases the latency of rapid eye movement (REM) sleep. Brain Research 278:308-12. [tarJAH, JMS]

Drucker-Colin, R., Bernal-Pedraza, J., Fernandez-Cancino, F. \& Oksenberg, A. (1984) Is vasoactive intestinal polypeptide (VIP) a sleep factor? Peptides 5:837-40. [WF]

Earnest, D. J. \& Sladek, C. D. (1984) Circadian rhythm in vasopressin release from rat suprachiasmatic explants in vitro. Socicty for Neuroscience Abstracts 10:500. [ta]A H]

Economo, C. von (1930) Sleep as a problem of localization. Journal of Nerious and Mental Disease 71:249-59. [HK]

Edwards, S. B. (1975) Autoradiographic studies of the projections of the midbrain reticular formation: Descending projections of nucleus cuneiformis. Journal of Comparative Neurology 161:341-58. [ta]AH]

Edwards, S. B. \& DeOlmos, J. S. (1976) Autoradiographic studies of the projections of the midbrain reticular formation: Ascending projections of nucleus cuneiformis. Journal of Comparative Neurology 165:417-32. [taJAH]

Eiden, L. E. G., Nilaver, G. \& Palkovitz, M. (1982) Distribution of vasoactive intestinal polypeptide (VIP) in the rat brain stem nuclei. Brain Research 231:472-77. [WF]

Elazar, Z. (1985) Epileptogenic effect of carbachol microinjected in the brainstem. Society for Neurosciences Abstracts 11:1314. [ZE]

Elazar, Z. \& Feldman, Z. (1986) Brainstem experimental seizures produced by microinjections of carbachol. Submitted for publication. [ZE]

Elazar, Z. \& Hobson, J. A. (1985) Neuronal excitability control in health and disease: A neurophysiological comparison of REM sleep and epilepsy. Progress in Neurobiology, in press. [tarJAH, ZE]

Emde, R. N. \& Metcalf, P. R. (1970) An electroencephalographic study of behavioral rapid eye movement states in the human newborn. Journal of Nervous and Mental Disease 150:376-86. [taJAH]

Evarts, E. V. (1960) Effects of sleep and waking on spontaneous and evoked discharge of single units in visual cortex. Federation Proceedings 19:82837. [taJAH]

(1964) Temporal patterns of discharge of pyramidal tract neurons during sleep and waking in the monkey. Journal of Neurophysiology 27:152-71. [ta]AH]

(1965) Relation of discharge frequency to conduction velocity in pyramidal tract neurons. Journal of Neurophysiology 28:216-28. [taJAH]

Everitt, B. J., Hokfelt, T., Terenius, L., Tatemoto, K., Mutt, V. \& Goldstein, M. (1984) Differential co-existence of neuropeptide Y (NPY). like immunoreactivity with catecholamine in the central nervous system of the rat. Neuroscience 11:443-62. [taJAH]

Faull, K. F., Zeller-DeAmicis, L. C., Radde, L., Bowersox, S. S., Baker, T. L., Kilduff, T. S. \& Dement, W. C. (1986) Biogenic amine concentrations in the brains of normal and narcoleptic canines: Current status. Sleep 9 , in press. [TSK]

Feinberg, I. (1974) Changes in sleep cycle patterns with age. Journal of Psychiatric Research 10:283-306. [SD]

Fernstrom, J. D., Fisher, L. A., Cusak, B. M. \& Gillis, M. A. (1980) Radioimmunologic detection and measurement of nonapeptides in the pineal gland. Endocrinology 106:243-51. [JRN]

Fishbein, W. \& Bright, P. F. (1986) Sexual dimorphism in sleep. In preparation. [WF]

Foote, S. L. (1973) Compensatory changes in REM sleep time of cats during adlibitum sleep and following brief REM sleep deprivation. Brain Research 54:261-76. [r]AH]

Foote, S. L., Aston-Jones, G. \& Bloom, F. E. (1980) Impulse activity of locus coeruleus neurons in awake rats and monkeys is a function of sensory stimulation and arousal. Proceedings of the National Academy of Sciences, USA 77:3033-37. [SLF]

Foote, S. L., Bloom, F. E. \& Aston-Jones, G. (1983) Nucleus locus ceruleus: New evidence of anatomical and physiological specificity. Physiological Reviews 63:844-914. [SLF, ta]AH, MS]

Fuller, C. A., Lydic, R., Sulzman, F. M., Albers, H. E., Tepper, B. \& Moore-Ede, M. C. (1981) Circadian rhythm of body temperature persists after suprachiasmatic lesions in the squirrel monkey. American Journal of Physiology 241:R385-91. [ta]AH]

Fung, S. J., Boxer, P. A., Morales, F. R. \& Chase, M. H. (1982) 
Hyperpolarizing membrane responses induced in lumbar motoneurons by stimulation of the nucleus reticularis pontis oralis during active sleep. Brain Rescarch 248:267-73. [ZE, taJAH]

Fuxe, K. (1965) Evidence for the existence of monoamine neurons in the central nervous system, 3. Nerve terminals. Zeitschrift für Zellforschung und Mikroskopische Anatomie 65:573-96. [taJAH]

French, T. \& Fromm, E. (1964) Dream interpretation: A new approach. Basic Books. [RG]

Friedman, L. \& Jones, B. E. (1984) Computer graphics analysis of sleepwakefulness state changes after pontine lesions. Brain Rescarch Bulletin 13:53-68. [BE], PJS]

Gallager, D. W. \& Aghajanian, G. K. (1976) Effect of antipsychotic drugs on the firing of dorsal raphe cells, 2. Reversal by picrotoxin. European Journal of Pharmacology 39:357-64. [taJAH]

Gallager, D. W. \& Pert, A. (1978) Afferents to brain stem nuclei (brain stem raphe, nucleus reticularis pontis caudalis and nucleus gigantocellularis) in the rat as demonstrated by microintophoretically applied horseradish peroxidase. Brain Rescarch 144:257-75. [ta]AH]

Gallistel, C. R. (1980) The organization of action: A new synthesis. Erlbaum. [ta]AH]

Gassel, M. M., Marchiafava, P. L. \& Pompeiano, O. (1964) Tonic and phasic inhibition of spinal reflexes during deep desynchronized sleep in unrestrained cats. Archives ltaliennes de Biologie 102:471-99. [taJAH]

George, R., Haslett, W. L. \& Jenden, D. J. (1964) A cholinergic mechanism in the brainstem reticular formation: Induction of paradoxical sleep. International Journal of Neurophannacology 3:541-52. [PAMvD]

Gillin, J. C. \& Borbelly, A. A. (1985) Sleep: A neurobiological window on affective disorders. Trends in Neuroscience 8:537-42. [rJAH]

Gillin, J. C., Sitaram, N., Janowsky, D., Risch, C., Huey, L. \& Storch, F. I. (1985) Cholinergic mechanisms in REM sleep. In: Sleep: Ncurotransmitters and neuromodulators, ed. A. Wauquier, J. M. Gaillard, J. M. Monti \& M. Radulovacki. Raven Press. [ta]AH]

Gillin, J. C., Sitaram, N., Wehr, T., Duncan, W., Post, R., Murphy, D. L., Mendelson, W. B., Wyatt, R. J. \& Bunney, W. E. (1984) Sleep and affective illness. In: Neurobiology of mood disorders, ed. R. M. Post \& J. C. Ballenger. Willians and Wilkins. [WBM]

Glazer, E. J., Steinbusch, H., Verhofstad, A. \& Basbaum, A. I. (1981) Serotonin neurons in nucleus raphe dorsalis and paragigantocellularis of the cat contain enkephalin. Journal of Physiology (Paris) 77:241-45. [taJAH]

Glenn, L. L. \& Dement, W. C. (1981a) Membrane potential, synaptic activity, and excitability of hindlimb motor neurons during wakefulness and sleep. Journal of Neurophysiology 46:839-54. [taJAH]

(1981b) Membrane resistance and rheobase of hindlimb motor neurons during wakefulness and sleep. Journal of Neurophysiology 46:1076-88. [taJAH]

(1985) Membrane potential and input resistance in alpha motoneurons of hindlimb extensors during isolated and clustered episodes of phasic events in REM sleep. Brain Rescarch 339:79-86. [taJAH]

Glemn, L. L., Hadda, J., Roy, J. P., Deschenes, M. \& Steriade, M. (1982) Anterograde tracer and field potential analysis of the neocortical layer 1 projection from nucleus ventralis medialis of the thalamus in the cat. Ncuroscicnce 7:1861-77. [ta]AH]

Coldberg, G. (1985) Supp;ementary motor area structure and function: Review and hypotheses. Bchavioral and Brain Sciences 8:567-616. [tnjAH]

Goldstein, R. (1983) A GABAergic habenulo-raphe pathway mediation of the hypnogenic effects of vasotocin in cat. Neuroscience 10:941-45. [JRN]

(1983) The administration of synthetic vasotocin into various spaces of the ventricular system of the brain and the sleep-wake cycle of the cat. Recue Roumaine de Neurologie 21:111-13. [JRN]

Graham-Brown, T. (1914) The intrinsic factors in the act of progression in the mammal. Proccedings of the Royal Society (Britain) 84:308-19. [taJAH]

Craybiel, A. M. (1977) Direct and indirect preoculomotor pathways of the brain stem: An autoradiographic study of the pontine reticular formation in the cat. Journal of Comparative Neurology 175:37-78. [taJAH]

Greene, R. W. \& Carpenter, D. O. (1981) Biphasic responses to acetylcholine in mammalian reticulospinal neurons. Collular and Molecular Neurobiology 1:401-05. [P]S]

(1985) Actions of neurotransmitters on pontine medial reticular formation of the cat. Journal of Neurophysiology 54:520-31. [ZE, rJAH, MS, PJS]

Greenberg, R. (1981) Dreams and REM sleep: An integrative approach. In: Slecp dreams and memory, ed. W. Fishbein. Spectrum Publications. [RC]

Greenberg, R. \& Pearlman, C. (1978) If Freud only knew: A reconsideration of psychoanalytic dream theory. International Review of Psychoanalysis 5:71-75. [RG]

Grillner, S. (1975) Some aspects on the descending control of the spinal circuits generating locomotor movements. In: Neural control of locomotion, ed. R. M. Herman, S. Grillner, P. S. G. Stein \& S. G. Stuart. Plenum Press. [r]AH]

Groos, G. (1984) The physiological organization of the circadian sleep-wake cycle. In: Experimental brain research, Supplement 8 , Sleep mechanisms, ed. A. Borbély \& J. L. Valatx. Springer. [ta]AH]

Gustafson, E. L. \& Moore, R. Y. (1985) Differential projections of neuropeptide $\mathrm{Y}$ containing neurones in the locus coeruleus of the rat. Neuroscience Abstracts 11:89. [MS]

Guyenet, P. G. \& Aghajanian, G. K. (1979) ACl, substance P, and metenkephalin in the locus coeruleus: Pharmacological evidence for independent sites of action. European Journal of Pharnacology 53:31928. [tajAH]

Haefely, W., Jalfre, M. \& Ruch-Munachon, M. A. (1975) A pathway and neurotransmitters involved in the control of noradrenergic locus coeruleus neurons. In: Chemical tools in catecholamine research, Vol. 2, ed. O. Almgren, A. Carlsson \& J. Engel. North-Holland. [WH]

Haefely, W., Ruch-Monachon, M. A., Jalfre, M. \& Schaffner, R. (1976) Interaction of psychotropic agents with central neurotransmitters as revealed by their effects on PGO waves in the cat. Drug Rescarch 26:710. [WH]

Haigler, H. J. \& Aghajanian, G. K. (1974) Lysergic acid diethylamide and serotonin: A comparison of effects on serotonergic neurons and neurons containing a serotonergic input. Journal of Pharmacology and Experimental Therapcutics 188:688-99. [taJAH]

Hanada, Y. \& Kawamura, H. (1981) Sleep-waking electrocorticographic rhythms in chronic cerveau isole rats. Physiology and Behavior 26:72528. [HK]

Heimer, L. (1978) The olfactory cortex and the ventral striatum. In: Limbic mechanisms: The continuing cuolution of the limbic concept, ed. K. E. Livingston \& O. Hornykiewicz. Plenum. [CHV]

Hendricks, J. C., Morrison, A. R. \& Mann, G. L. (1982) Different behaviors during paradoxical sleep without atonia depend upon pontine lesion site. Brain Rescarch 239:81-105. [taJAH]

Hendry, S. H., Jones, E. G. \& Graham, J. (1979) Thalamic relay nuclei for cerebellar and certain related fiber systems in the cat. Journal of Comparatice Netrology 185:679-714. [taJAH]

Henley, K. \& Morrison, A. R. (1974) A re-evaluation of the effects of lesions on the pontine tegmentum and locus coeruleus on phenomena of paradoxical sleep in cat. Acta Neurobiologiae Experimentalis (Warsaw) 34:215-32. [tajAH]

Henn, V. (1980) Reticulo-oculomotor neurons: Specificity of discharge in relation to eye movements. Neuroscience Research Program Bulletin 18:55-58. [taJAH]

Henn, V., Baloh, R. W. \& Hepp, K. (1984) The sleep-wake transitions in the oculomotor system. Experimental Brain Research 54:166-76. [ta]AH]

Henn, V., Buttner-Ennever, J. A. \& Hepp, K. (1982) The primate oculomotor system, 1. Motoneurons: A synthesis of anatomical, physiological, and clinical data. Human Neurobiology 1:77-85. [taJAH]

Henn, V., Hepp, K. \& Buttncr-Ennever, J. A. (1982) The primate occulomotor system, 2. Premotor systems: A synthesis of anatomical, physiological, and clinical data. Human Ncurobiology 1:87-95. [taJAH]

Hernández-Peón, R., Chavez-Ibarra, G., Morgane, P. J. \& Timo-laria, C. (1962) Cholinergic pathways for sleep, alertness and rage in the limbic midbrain circuit. Acta Neurologica Latinoamericana 8:93-96. [PJM]

(1963) Limbic cholinergic pathways involved in sleep and emotional behavior. Experimental Neurology 8:93-111. [PJM]

Hess, W. R. (1931) Le sommeil. Comptes Rendus de Biologie 107:1333-64. [WBM, taJAH]

Heule, F., Lorez, H., Cumin, R. \& Haefely, W. (1983) Studies on the anticonflict effect of midazolam injected into the amygdala. Neuroscience Letters. Suppl. 14:164. [WH]

Heym, J., Steinfels, G. F. \& Jacobs, B. L. (1982) Activity of serotonincontaining neurons in the nucleus raphe pallidus of freely moving cats. Brain Research 251:259-76. [taJAH]

(1984) Chloral hydrate anesthesia alters the responsiveness of central serotonergic neurons in the cat. Brain Rescarch 291:63-72. [ta]AH]

Hirsch, J. C., Fourment, A. \& Marc, M. E. (1983) Sleep-related variations of membrane potentials in the lateral geniculate body relay neurons of the cat. Brain Research 259:308-12. [taJAH]

Hobson, J. A. (1965) The effects of chronic brain-stem lesions on cortical and muscular activity during sleep and waking in the cat. Elcctroencephalography and Clinical Ncurophysiology 19:41-62. [HK]

(1974) The cellular basis of sleep cycle control. In: Advances in slecp research, vol. 1, ed. E. Weitzman. Spectrum. [rJAH]

(1978) What is a behavioral state? In: Neuroscience symposia, vol. 3, Aspects of behavioral neurobiology, ed. J. A. Ferrendelli. Society for Neuroscience. [taJAH] 
(1983a) Sleep mechanisms and pathophysiology: Some clinical implications of the reciprocal interaction hypothesis of sleep cycle control. Psychosomatic Medicine 45:123-40. [ta]AH]

(1983b) Sleep: Order and disorder. Behavioral Biology and Medicine 1:136. [rJAH]

(1984) How does the cortex know when to do what? A neurobiological theory of state control. In: Dynamic aspects of neocortical function, ed. C. M. Edelman, W. E. Gall \& M. W. Cowan. Neurosciences Research Foundation. [taJAH]

Hobson, J. A., Goldberg, M., Vivaldi, E. \& Riew, D. (1983) Enhancement of desynchronized sleep signs after pontine microinjection of the muscarinic agonist bethanechol. Brain Research 275:127-36. [ta]AH]

Hobson, J. A. \& McCarley, R. W. (1972) Spontaneous discharge rates of cat cerebellar Purkinje cells in sleep and waking. Electroencephalography and Clinical Neurophysiology 33:457-69. [rJAH]

(1977) The brain as a dream state generator: An activation synthesis hypothesis of the dream process. American Journal of Psychiatry 134:1335-48. [RG]

Hobson, J. A., McCarley, R. W., Freedman, R. \& Pivik, R. T. (1974) Timecourse of discharge rate changes in cat pontine brainstem neurons during the sleep cycle. Journal of Neurophysiology 37:1297-1309. [ta]AH]

Hobson, J. A., McCarley, R. W. \& Nelson, J. P. (1983) Location and spiketrain characteristics of cells in anterodorsal pons having selected decreases in firing rate during desynchronized sleep. Journal of Neurophysiology 50:770-83. [ta]AH]

Hobson, J. A., McCarley, R. W., Pivik, R. T. \& Freedman, R. (1974) Selective firing by cat pontine brainstem neurons in desynchronized sleep. Journal of Neurophysiology 37:497-510. [taJAH]

Hobson, J. A., McCarley, R. W. \& Wyzinski, P. W. (1975) Sleep cycle oscillation: Reciprocal discharge by two brainstem neuronal groups. Science 189:55-58. [BE], taJAH]

Hobson, J. A., McCarley, R. W., Wyzinski, P. W. \& Pivik, R. T. (1973) Reciprocal tonic firing by FTG and LC neurons during the sleep-waking cycle. Slecp Research 2:29. [ta]AH]

Hobson, J. A. \& Steriade, M. (1986) The neuronal basis of behavioral state control. In: Handbook of physiology. Section I, "The Nervous System," ed. V. Mountcastle. Intrinsic Regulatory Systems of the Brain. American Physiological Society 4: 701-823. [taJAH]

Hokfelt, T., Ljungdahl, A., Steinbusch, H., Verhofstad, A., Nilsson, G., Brodin, E., Pernow, B. \& Goldstein, M. (1978) Immunocytochemical evidence of substance P-like immunoreactivity in some 5-HT-containing neurons in the cat central nervous system. Neuroscience 3:51738. [taJAH]

Holst, E. von \& Mittelstaedt, H. (1950) Das reafferenzprinzip. Die Naturwissen 20:464-76. [taJAH]

Hoshino, K. \& Pompeiano, O. (1976) Selective discharge of pontine neurons during the postural atonia produced by an anticholinesterase in the decerebrate cat. Archices Italiennes de Biologie 114:244-47. [ta]AH]

Hösli, L., Tebecis, A. K. \& Schonwetter, H. P. (1971) A comparison of the effects of monoamines on neurones of the bulbar reticular formation. Brain Research 25:357-70. [MS]

Hounsgaard, J. \& Kiehn, O. (1985) $\mathrm{Ca}^{++}$-dependent bistability induced by serotonin in spinal motoneurons. Experimental Brain Research 57:42225. $[P A M v D]$

Huttenlocher, P. R. (1961) Evoked and spontaneous activity in single units of medial brain stem during natural sleep and waking. Journal of Ncurophysiology 24:451-68. [ta]AH]

Inoue, S. \& Borbelly. A. A., eds. (1985) Endogenous sleep substances and sleep regulation. Japan Scientific Societies Press. [AAB]

Inoue, S., Honda, K. \& Komoda, Y. (1985) Sleep-promoting substances. In: Sleep: Neurotransmitters and neuromodulators, ed. A. Wauquier, J. M. Gaillard, J. M. Monti \& M. Radulovacki. Raven Press. [taJAH]

Inouye, S. T. \& Kawamura, H. (1979) Persistence of circadian rhythmicity in a mammalian hypothalamic "island" containing suprachiasmatic nucleus. Proceedings of the National Academy of Science (USA) 76:5962-66. [tarJAH]

Inubushi, S., Kobayashi, T., Oshima, T. \& Torü, S. (1978) An intracellular analysis of EEG arousal in cat motor cortex. Japanese Journal of Physiology 28:689-708. [MS]

Ito, K. \& McCarley, R. W. (1984) Alterations in membrane potential and excitability of cat medial pontine reticular formation neurons during changes in naturally occurring sleep-wake states. Brain Research 292:169-75. [WF, ta]AH]

Ito, M. (1975) The control mechanisms of cerebellar motor systems. In: The neurosciences: Third study program, ed. F. O. Schmitt \& F. G. Worden. MIT, [JA]

Jalfre, M., Ruch-Monachon, M. A. \& Haefely, W. (1974) Method for assessing the interaction of agents with 5-hydroxytryptamine neurons and receptors in the brain. Advances in Biochemical Psychopharmacology 10:121-34. [WH]

Janowsky, D., El-Yousef, M. K., Davis, J. M. \& Sederke, H. J. (1972) A cholinergic-adrenergic hypothesis of mania and depression. Lancet 2:62335. [ta]AH]

Jasper, H. H. \& Tessier, J. (1971) Acetylcholine liberation from cerebral cortex during paradoxical (REM) sleep. Science 172:601-2. [MS]

Jeannerod, M., Monet, J. \& Jouvet, M. (1965) Effects secondaires de la deafferentation visuelle sur l'activité electrique phasique ponto-geniculooccipitale du sommeil paradoxical. Journal of Physiology (Paris) 57:25556. [taJAH]

Johnson, E. S., Roberts, M. H. T. \& Straughan, D. W. (1969) The responses of cortical neurones to monoamines under differing anesthetic conditions. Journal of Physiology (London) 203:261-80. [CHV]

Johnston, M. V., McKinney, M. \& Coyle, J. T. (1979) Evidence for a cholinergic projection to neocortex from neurons in basal forebrain. Proceedings of the National Academy of Sciences of the United States of America 79:5392-96. [CHV]

Jones, B. E. (1979) Elimination of paradoxical sleep by lesions of the pontine gigantocellular tegmental field in the cat. Neturoscience Letters 13:28593. [ta]AH]

(1980) Projections from the pontine gigantocellular tegmental field in the rat. Neuroscience Abstracts 6:59. [BE]]

(1985a) Paradoxical sleep and chemically identified neurons of the reticular formation. In: Sleep: Neurotransmitters and neuromodulators, ed. A. Waquier, J. M. Gaillard, J. M. Monti \& M. Radulovacki. Raven Press. [taJAH, KS, PJS]

(1985b) Neuroanatomical and neurochemical substrates of mechanisms underlying paradoxical sleep. In Brain mechanisms of sleep, ed. D. J McGinty, P. L. Parmeggiani, R. Drucker-Colin \& A. Morrison. Raven Press. [JMS]

Jones, B. E. \& Friedman, L. (1983) Atlas of catecholamine perikarya, varicosities and pathways in the brainstem of the cat. Journal of Comparative Neurology 215:382-96. [BEJ]

Jones, B. E., Halaris, A. E., Mcllhany, M. \& Moore, R. Y. (1977) Ascending projections of the locus coeruleus in the rat, 1. Axonal transport in central noradrenaline neurons. Brain Research 127:1-21. [ta]AH]

Jones, B. E., Harper, S. T. \& Halaris, A. E. (1977) Effects of locus coeruleus lesions upon cerebral monoamine content, sleep-wakefulness states and the response to amphetamine in the cat. Brain Research 124:473-96. [BE], JMS]

Jones, B. E. \& Moore, R. Y. (1977) Ascending projections of the locus coeruleus in the rat, 2. Autoradiographic study. Brain Research 127:2353. [taJAH]

Jones, B. E. \& Yang, T.-Z. (1985) The efferent projections from the reticular formation and the locus coeruleus studied by anterograde and retrograde axonal transport in the rat. Journal of Comparative Neurology 242:56-92. [BE]]

Jones, E. G. \& Leavitt, R. Y. (1974) Retrograde axonal transport and the demonstration of nonspecific projections to the cerebral cortex and striatum from thalamic intralaminar nuclei in the rat, cat and monkey. Journal of Comparative Neurology 154:349-78. [ta]A H]

Jones, R. S. G. (1982) Responses of cortical neurones to stimulation of the nucleus raphe medianus: A pharmacological analysis of the role of indoleamines. Neuropharmacology 21:511-20. [CHV]

Jouvet, M. (1962) Recherches sur les structures nerveuses et les mécanismes responsables des differentes phases du sommeil physiologique. Archives Italiennes de Biologie 100:125-206. [taJAH]

(1969) Biogenic amines and the states of sleep. Science 163:32-41. [taJAH, HK, PJM]

(1972) The role of monoamine and acetylcholine-containing neurons in the regulation of the sleep-wake cycle. Ergebnisse der Physiologie 64:166307. [taJAH]

(1978) Does a genetic programming of the brain occur during paradoxical sleep? In: Cerebral correlates of conscious experience, ed. P. Buser \& A. Rougeul-Buser. Elsevier. [taJAH]

(1984) Indolamines and sleep-inducing factores. In: Slcep mechanisms, ed. A. A. Borbély \& J. L. Valatx. Experimental Brain Research, Suppl. 8, Springer. [rAAB]

Jouvet, M., Bobillier, P., Pujol, J. F. \& Renault, J. (1966) Effets des lesions du systéme du raphé sur le sommeil et la serotonine cerebrale. Comptes Rendus de la Societé de Biologie (Paris) 160:2343-50. [WBM]

Jouvet, M. \& Delorme, F. (1965) Locus coerulus et sommeil paradoxical. Comptes Rendus de la Societé de Biologie 159:895-99. [taJAH]

Jouvet, M. \& Michel, F. (1959) Correlations électromyographiques du sommeil chez le chat décortique et mésencephalique chronique. Comptes Rendus de la Societé de Biologie (Paris) 153:422-25. [ta]AH]

Kanamori, N., Sakai, K. \& Jouvet, M. (1980) Neuronal activity specific to 
paradoxical sleep in the ventromedial medullary reticular formation of unrestrained cats. Brain Research 189:251-55. [ta]AH, KS]

Karczmar, A. G., Longo, V. G. \& De Carolis, A. S. (1970) A pharmacological model of paradoxical sleep: The role of cholinergic and monoamine systems. Physiology and Bchavior 5:175-82. [taJAH]

Kasamatsu, T. (1970) Maintained and evoked unit activity in the mesencephalic reticular formation of the freely behaving cat. Experimental Neurology 28:450-70. [ta]AH]

Katayama, Y., DeWitt, D. S., Becker, D. P. \& Hayes, R. L. (1984) Behavioral evidence for cholinoceptive pontine inhibitory area: Descending control of spinal motor output and sensory input. Brain Rescarch 296:241-62. [JMS]

Keller, E. L. (1974) Participation of medial pontine reticular formation in eye movement generation in the monkey. Journal of Neurophysiology 37:316-32. [taJAH]

Kilduff, T. S., Bowersox, S., Kaitin, K., Baker, T. L., Ciaranello, R. D. \& Dement, W. C. (1986) Muscarinic cholinergic receptors and the canine model of narcolepsy. Slccp 9 , in press. [TSK]

Kimura, H., McGeer, P. L., Peng, J. H. \& McGeer, E. G. (1981) The central cholinergic system studied by choline acetyltransferase immunohistochemistry in the cat. Journal of Comparative Neurology 200:151-201. [BEJ, JMS, PJS]

Kimura, H. \& Maeda, T. (1982) Aminergic and cholinergic systems in the dorsolateral pontine tegmentum. Brain Rescarch 9:403-99. [PJS]

Klein, D. C. (1983) Converging circadian rhythms could explain effects of the pineal gland: Implications for psychiatric disease. In: Psychobiology and psychopathology. vol. 2: Circadian rhythms in psychiatry, ed. T. A. Wehr \& F. K. Goodwin. Boxwood Press. [JRN]

Kornhuber, H. H. (1975) Cerebral cortex, cerebellum and basal ganglia: An introduction to their motor functions. In: The neurosciences: Third study program, ed. F. O. Schmitt \& F. G. Worden.MIT [JA]

Kostowski, W., Giacalone, E., Garattini, S. \& Valzelli, L. (1968) Studies on behavioural and biochemical changes in rats after lesions of midbrain raphe. European Journal of Pharmacology 4:371-76. [WBM]

Kraemer, H. C., Hole, W. T. \& Anders, T. F. (1984) The detection of behavioral state cycles and classification of temporal structure in behavioral states. Slecp 7:3-17. [taJAH]

Krnjevic, K. (1974) Chemical nature of synaptic transmission in vertebrates. Physiological Roviews 43:418-540. [CHV]

Krnjević, K., Pumain, R. \& Renaud, L. (1971) The mechanism of excitation by acetylcholine in the cerebral cortex. Journal of Physiology (London) 215:247-68. [MS]

Kubo, T. \& Misu, Y. (1981a) Changes in arterial blood pressure after microinjections of nicotine into the dorsal area of the medulla oblongata of the rat. Ncuropharmacology 20:521-24. [PJS]

(1981b) Cardiovascular response to microinjection of physostigmine and choline into the dorsal medullary site of the rat. Neuropharmacology 20:1091-95. [PJS]

Kubota, K. \& Kidokoro, Y. (1965) Excitability of the membrane of lumbar motor ncurons and natural slecp in the cat. Japancse Journal of Physiology 16:217-26. [taJAH]

Kuhn, T. S. (1962) The structure of scientific revolutions. University of Chicago Press. [rJAH]

Kulikowski, J. J. (1971) Information channels of the senses. In: Bionics: The nervous system as a control system, ed. R. Gawronski. Elsevier. [PAMvD]

Kupfer, D. J., Spyker, D. G., Coble, P. \& McPortland, R. J. (1978) Amitryptyline and EEG sleep in depressed patients, 1. Drug effect. Slccp 1:149-59. [SD]

Lamour, Y., Dutar, P. \& Jobert, A. (1983) Effects of neuropeptides on rat cortical neurons: Laminar distribution and interaction with the effect of acetylcholine. Neuroscience 10:107-17. [MS]

Laurent, J. P., Cespuglio, R. \& Jouvet, M. (1972) Delimitation des voies ascendantes responsables de l'activité ponto-géniculo-occipitale chez le chat. Expericntia 28:1174-75. [RC]

(1974) Delimitation des voies ascendantes de l'activité ponto-géniculooccipitale chez le chat. Brain Research 65:29-52. [RC]

Lavie, P. \& Kripke, D. F. (1981) Ultradian circa 11-hour rhythms: A multioscillatory system. Life Scicnces 29:2445-50. [taJAH]

Leger, L., Degueurce, A., Lundberg, J. J., Pujol, J. F. \& Møllgård, K. (1983) Origin and influence of the serotoninergic innervation of the subcommissural organ in the rat. Neuroscience 10:411-23. [JRN]

Leger, L., Wiklund, L., Descarries, L. \& Person, M. (1979) Description of an indolaminergic cell component in the cat locus coeruleus: A fluorescence histochemical and radioautographic study. Brain Research 168:43-56. [ta]AH]

Lehmann, J., Nagy, J. I., Atmadja, S. \& Fibiger, H. C. (1980) The nucleus basalis magnocellularis: The origin of a cholinergic projection to the neocortex of the rat. Neuroscience 5:1161-74. [CHV]

Lendrem, D. W. (1984) Sleeping and vigilance in birds, 2. An experimental study of the Barbary dove (Streptopelia risoria). Animal Behaviour $32: 243-48$. [SD]

Lidbrink, P. (1974) The effect of lesions of ascending noradrenaline pathways on sleep and waking in the rat. Brain Research 74:19-40. [JMM]

Llinas, R. \& Jahnsen, H. (1982) Electrophysiology of manmalian thalamic neurons in vitro. Nature 297:406-8. [WBM]

Llinas, R., Yarom, Y. \& Sugimori, M. (1981) Isolated mammalian brain in vitro: New technique for analysis of electrical activity of neuronal circuit function. Federation Proceedings 40:2240-45. [MS]

Lund, J. S., Lund, R. D., Henrickson, A. E., Bunt, A. H. \& Fuchs, A. F. (1975) The origin of efferent pathways from the primary visual cortex, area 17, of the macaque monkey as shown by retrograde transport of horseradish peroxidase. Journal of Comparative Neurology 164:287-304. [tajAH]

Lundberg, J. M., Hokfelt, T., Schultzberg, M., Uvnas-Wallenstein, K., Kohler, C., \& Said. S. I. (1979) Occurrence of vasoactive intestinal polypeptide (VIP)-like immunoreactivity in certain cholinergic neurons of the cat: Evidence from combined immunohistochemistry and acetylcholinesterase staining. Journal of Neuroscience 4:1539-59. [WF]

Lydic, R., McCarley, R. W. \& Hobson, J. A. (1982) Multiple sleep cycle averaging of dorsal raphe activity. Sleep Research 11:22. [r]AH]

(1983a) The time-course of dorsal raphe discharge, PCO waves, and muscle tone averaged across multiple sleep cycles. Brain Research 274:365-70. [taJAH]

(1983b) Enhancement of dorsal raphe discharge by medial pontine reticular formation stimulation depends on behavioral state. Neuroscience Letters 38:35-40. [taJAH]

(1984) Forced activity alters sleep cycle periodicity and dorsal raphe discharge rhythm. American Journal of Physiology 247:R 135-45. [SD, tarJAH]

(1985) Timing function of the dorsal raphe nucleus and the temporal organization of the ultradian sleep cycle. In: Expcrimental brain rescarch, Supplement 12, Ultradian rhythms in physiology and behavior, ed. $\mathrm{H}$. Schultz \& P. Lavie. Springer. Pp. 125-144. [ta]AH]

(1986a) Forced locomotor activity alters the temporal organization of sleep: The discharge frequency of dorsal raphe neurons and PGO waves. Manuscript submitted. [tar]AH]

(1986b) Forced locomotor activity alters the temporal organization of sleep: The time course profiles of dorsal raphe neuronal discharge, PGO waves, and behavioral states. Manuscript submitted. [tarJAH]

Lydic, R. \& Orem, J. (1979) Respiratory neurons of the pneumotaxic center during sleep and wakefulness. Neuroscience Letters 15:187-92. [taJAH]

McCarley, R. W. (1978) Control of sleep-waking state alterations in Felis domesticus. In: Neuroscience symposia, vol. 3, Aspects of behavioral neurobiology. Society for Neuroscience. [tajAH]

(1980) Pontine brainstem neuronal activity and REM sleep control mechanisms. Proceedings of the 28th International Congress of Physiological Sciences, Hungarian Academy of Scicnce, Advances in Physiological Science 1:293-99. [taJAH]

(1982) REM sleep and depression: Common neurobiological control mechanisms. American Journal of Psychiatry 139:565-70. [taJAH]

McCarley, R. W. \& Hobson, J. A. (1971) Single neuron activity in cat gigantocellular tegmental field: Selectivity of discharge in desynchronized sleep. Science 174:1250-52. [BE]]

(1975a) Discharge patterns of cat pontine brainstem neurons during desynchronized sleep. Journal of Neurophysiology 38:751-66. [taJAH]

(1975b) Neuronal excitability over the sleep cycle: A structural and mathematical model. Science 189:58-60. [AAB, tar]AH, BE], PJS]

McCarley, R. W. \& Hoffman, E. (1981) REM sleep dreams and the activation-synthesis hypothesis. American Journal of Psychiatry 138:90412. [taJAH]

McCarley, R. W. \& Ito, K. (1983) Intracellular evidence linking medial pontine reticular formation neurons to $\mathrm{PCO}$ wave generation. Brain Research 280:343-48. [WF, tajAH]

(1985) Desynchronized sleep-specific changes in membrane potential and excitability in medial pontine reticular formation neurons: Implications for concepts and mechanisms of behavioral state control. In Brain mechanisms of slecp, ed. D. J. McGinty, R. Drucker-Colin, A. Morrison \& P. L. Parmeggiani. Raven Press. [ZE]

McCarley, R. W. \& Massaquoi, S. (1985) A limit cycle model of the ultradian rhythm of REM sleep. In: Experimental brain research, Supplement 12, Ultradian rhythms, ed. H. Schultz \& P. Lavie. Springer. [JA, tarJAH]

McCarley, R. W. \& Massaquoi, S. (in press) A limit cycle mathematical model of the REM sleep oscillator system. American Journal of Physiology. [r]AH] 
McCarley, R. W., Nelson, J. P. \& Hobson, J. A. (1978) Ponto-geniculooccipital (PGO) burst neurons: Correlative evidence for neuronal generators of PGO waves. Science 201:269-72. [ta]AH]

McCarley, R. W., Winkelman. J. W. \& Duffy, F. H. (1983) Human cerebral potentials associated with REM sleep rapid eye movements: Link to PGO waves and waking potentials. Brain Research 274:359-64. [JA]

McGinty, D. J. (1985) Physiological equilibrium and the control of sleep states. In: Brain mechanisins of sleep, ed. D. J. McGinty, R. DruckerColin, A. Morrison \& P. L. Parmeggiani. Raven Press. [SJH, JMS, PJS]

McGinty, D. J. \& Harper, R. M. (1972) 5-HT-containing neurons: Unit activity during sleep. Sleep Research 1:27. [rJAH]

(1976) Dorsal raphe neurons: Depression of firing during sleep in cats. Brain Research 101:569-75. [tarJAH]

McGinty, D. J., Harper, R. M. \& Fairbanks, M. K. (1974) Neuronal unit activity and the control of sleep states. In: Advances in slecp research, ed. E. D. Weitzman. Spectrum Publications. [JMS]

McKenna, T., McCarley, R. W. \& Hobson, J. A. (1975) Pontine brain stem unit activity during naturally occurring desynchronized sleep episodes and their carbachol-induced analogues. Sleep Research 4:49. [taJAH]

Madison, D. V. \& Nicoll, R. A. (1982) Noradrenaline blocks accommodation of pyramidal cell discharge in the hippocampus. Nature (London) 299:636-38. [MS]

Maeda, T., Pin, C., Salvert, D., Ligier, M. \& Jouvet, M. (1973) Les neurones contenant des catecholamines du tegmentum pontique et leur voies de projection chez le chat. Brain Research 57:119-52. [taJAH]

Mancia, M. (1969) EEG and behavioral changes owing to splitting of the brainstem in cats. Electroencephalography and Clinical Neurophysiology 27:487-503. [taJAH]

Martin, R. F., Jordan, L. M. \& Willis, W. D. (1978) Differential projections of cat medullary raphe neurons demonstrated by retrograde labelling following spinal cord lesions. Journal of Comparative Neurology 182:7788. [taJAH]

Masserano, J. M. \& King, C. (1982a) Effects on sleep of acetylcholine perfusion of the locus coenuleus of cats. Neuropharnacology 21:1163-67. [tajAH]

(1982b) Effects on sleep of phentolamine and epinephrine infused into the locus coeruleus of cat. European Journal of Phannacology 84:199-204. [tajAH]

May, R. M. (1973) Stability and competition of model ecosystems. Princeton University Press. [taJAH]

Meader, A. (1916) The dream problem. Nervous and mental disease monograph no. 22. New York Publishing Co. [RG]

Mefford, I. N., Baker, T. L., Boehme, R., Foutz, A. S., Ciaranello, R. D., Barchas, J. D. \& Dement, W. C. (1983) Narcolepsy: Biogenic amine deficits in an animal model. Science 220:629-32. [tajAH]

Mergner, T. \& Pompeiano, O. (1978) Single unit firing patterns in the vestibular nuclei related to saccadic eye movements in the decerebrate cat. Archives Italiennes de Biologie 116:91-119. [taJAH]

Mesulam, M.-M., Mufson, E. J., Levey, A. I. \& Wainer, B. H. (1984) Atlas of cholinergic neurons in the forebrain and upper brainstem of the macaque based on monoclonal choline acetyltransferase immunohistochemistry and acetylcholinesterase histochemistry. Neuroscience 12:669-86. [ta]AH]

Mesulam, M. M., Mufson, E. J., Wainer, B. H. \& Levey, A. I. (1983) Central cholinergic pathways in the rat: An overview based on an alternative nomenclature (Chl-Ch6). Neuroscience 10:1185-1201. [PJS]

Michaelis, R. \& Hoffimann, E. (1973) Zur Phänomenologie und Ätiopathogenese der Hypersomnie bei endogen phäsischen Depressionen. In: The nature of slecp, ed. U. J. Janonovic. Gustav Fischer Verlag. [WBM]

Mitler, M. M. \& Dement, W. C. (1974) Cataplectic-like behavior in cats after microinjection of carbachol in pontine reticular formation. Brain Research 69:335-43. [ta]AH]

Monachon, M. A., Burkard, W. P., Jalfre, M. \& Haefely, W. (1972) Blockade of central 5-hydroxytryptamine receptors by methiothepin. Archives of Pharmacology 274:192-97. [WH]

Monaco, A. P., Baghdoyan, H. A., Nelson, J. P. \& Hobson, J. A. (1984) Cortical wave amplitude and eye movement direction are correlated in REM sleep but not in waking. Archices laliennes de Biologie 122:21323. [JA, RC, taJAH]

Monti, J. M. (1983) Catecholamines and the sleep-wake cycle. II. REM sleep. Life Sciences 32:1401-15. [JMM]

Monti, J. M., Pellejero, T., Jantos, H., D'Angelo, L., Barbeito, L. \& Abó, V. (submitted) Sleep-wake cycle and sensitivity to adrenoceptor agonists in the rat after DSP-4, a selective noradrenergic neurotoxin. [JMM]

Monti, J. M., Pellejero, T., Jantos, H. \& Pazos, S. (1985) Role of histamine in the control of sleep and waking. In: Sleep: Neurotransinitters and neuromodulators, ed. A. Wauquier, J. M. Monti, J. M. Gaillard \& M. Radulovacki. Raven Press. [JMM]

Moore, R. Y. (1982) The suprachiasmatic nucleus and the organization of a circadian system. Trends in Neuroscience 5:404-7. [ta]AH]

Moore, R. Y. \& Eichler, V. B. (1972) Loss of circadian adrenal corticosterone rhythm following suprachiasmatic lesions in the rat. Brain Rescarch 42:201-6. [rJAH]

Moore, R. Y., Halaris, A. E. \& Jones, B. E. (1978) Serotonin neurons of the midbrain raphe: Ascending projections. Journal of Comparative Neurology 180:417-38. [taJAH]

Morales, F. \& Chase, M. H. (1978) Intracellular recording of lumbar motoneuron membrane potential during sleep and wakefulness. Expcrimental Neurology 62:821-27. [ta]AH]

Morgane, P. J. \& Jacobs, M. S. (1979) Raphe projections to the locus coeruleus in the rat. Brain Rescarch Bullctin 4:519-34. [ta]AH]

Morgane, P. J. \& Stern, W. C. (1974) Chemical anatomy of brain circuits in relation to sleep and wakefulness. In: Adeances in slecp rescarch, vol. 1 , ed. E. Weitzman. Spectrum. [taJAH]

Mori, S., Ohta, Y., Matsuyama, K. \& Takakusaki, K. (1986) The setting of postural muscle tone: Its importance for behavior in freely moving, intact cats. In: Neuronal and endogenous chemical control mechanisms on Emotional Bchavior, ed. Y. Oomura. Japan Scientific Socictics/Springer. [SM]

Mori, S., Sakamoto, T. \& Ohta, Y. (1986) Neuronal mechanisms underlying plastic postural changes in decerebrate, reflexively standing cats. In: Neuronal mechanisms of conditioning, ed. C. D. Woody \& D. L. Alkon. Plenum. [SM]

Morilak, D. A. \& Jacobs, B. L. (1985) Noradrenergic modulation of sensorimotor processes in intact rats: The masseteric reflex as a model system. Journal of Neuroscience 5:1300-1306. [rJAH]

Morrison, A. R. (1979) Brainstem regulation of behavior during sleep and wakefulness. Progress in Neurobiology and Physiological Psychology 8:91-131. [taJAH]

(1983) A window on the sleeping brain. Scientific American 248:94-103. [ta]AH]

Morrison, A. R. \& Pompeiano, O. (1965a) An analysis of the supraspinal influences acting on motoneurons during sleep in the unrestrained cat. Responses of the alpha motoneurons to direct electrical stimulation during sleep. Archices Italiennes de Biologie 103:497-516. [ta]AH]

(1965b) Central depolarization of group la afferent fibers during desynchronized sleep. Archives Italiennes de Biologic 103:517-37. [ta]AH]

(1965c) Pyramidal discharge from somatosensory cortex and cortical control of primary afferents during sleep. Archives Italiennes de Biologic 103:538-68. [taJAH]

Moruzzi, G. (1960) Synchronizing influences of the brain stem and the inhibitory mechanisms underlying the production of sleep by sensory stimulation. Electroencephalography and Clinical Neurophysiology Supplement 13:231-56. [taJAH]

Moruzzi, G. \& Magoun, H. W. (1949) Brainstem reticular formation and activation of the EEG. Electroencephalography and Clinical Neurophysiology 1:455-73. [ta]AH, PJS]

Mosco, S. S., Haubrich, D. \& Jacobs, B. L. (1977) Serotonin afferents to the dorsal raphe nucleus: Evidence from HRP and synaptosomal uptake studies. Brain Research 119:269-90. [ta]AH]

Moss, M. S., Glazer, E. J. \& Basbaum, A. I. (1981) Enkephalinimmunoreactive perikarya in the cat raphe dorsalis. Neuroscience Letters 21:33-37. [taJAH]

Mouret, J., Coindet, J. \& Chouvet, G. (1974) Effet de la pinealectomie sur les états et rythmes de sommeil du rat malé. Brain Research 81:97-105. [JRN]

Nagatsu, I., Inagaki, S., Kondo, Y., Karasawa, N. \& Nagatsu, T. (1979) Acto Histochemica Cytochemica 12:20-37. [JMM]

Nakamura, S. (1975) Two types of inhibitory effects upon brain stem reticular neurons by low frequency stimulation of the raphe nucleus in the rat. Brain Research 3:140-44. [ta]AH]

Nakata, K. \& Kawamura, H. (1986) ECoG sleep-waking rhythms and bodily activity in the cerveau isole rat. Physiology and Behavior, in press. [HK]

Nauta, W. J. H. (1946) Hypothalamic regulation of sleep in rats: An experimental study. Journal of Neurophysiology 9:285-316. [HK]

Nauta, W. J. \& Kuypers, G. J. M. (1958) Some ascending pathways in the brain stem reticular formation. In: Reticular formation of the brain, ed. H. Jasper, L. D. Proctor, R. S. Knighton, W. S. Noshay \& R. T. Costello. Little, Brown. [taJAH]

Nelson, J. P., McCarley, R. W. \& Hobson, J. A. (1978) PGO burst neurons, 4. State-dependent stimulation from lateral geniculate nucleus. Slecp Research 7:38. [taJAH] 
(1983) REM sleep burst neurons, PGO waves, and eye movement information. Journal of Ncurophysiology 50:784-97. [JA, taJAH]

Netick, A., Orem, J. \& Dement, W. C. (1977) Neuronal activity specific to REM sleep and its relationship to breathing. Brain Research 120:197207. [ta]AH, KS]

Nicoll, R. A. \& Madison, D. V. (1982) General anesthetics hyperpolarize neurons in the vertebrate central nervous system. Science 217:1055-57. [ta]A H]

Nishikawa, T. \& Scatton, B. (1983) Evidence for a GABAergic inhibitory influence on serotonergic neurons originating from the dorsal raphe. Brain Rescarch 279:325-29. [ta]AH]

Normanton, J. R. \& Gent, J. P. (1983) Comparison of the effects of two "sleep" peptides, delta sleep-inducing peptide and arginine-vasotocin, on single neurons in the rat and rabbit brain stem. Neuroscience 8:107-14. [JRN]

North, R. A. \& Yoshimura, M. (1984) The actions of noradrenaline on neurones of the rat substantia gelatinosa in vitro. Journal of Physiology (London) 349:43-56. [PAMvD]

Ohta, Y., Nonaka, S. \& Mori, S. (1985) The suppression of postural muscle tone and its brainstem and spinal cord neuronal mechanisms in acute decerebrate, reflex standing cats. Neuroscience Letters Supplement 1:S66. [SM]

Ohta, Y., Sakamoto, T. \& Mori, S. (1984) The cells of origin projecting their axons to the dorsal part of the central tegmental field in the pons. Japancse Journal of Physiology 46:377. [SM]

(1985) Inhibitory effects of medial reticular neurons in the medulla oblongata upon alpha-motoneurons innervating hindlimb extensor muscles. Japanese Journal of Physiology 47:430. [SM]

Olpe, H. R., Jones, R. S. \& Steinmann, M. W. (1983) The locus coeruleus: Actions of psychoactive drugs. Experientia 39:242-49. [PJS]

Orem. J. (1980) Medullary respiratory neuron activity: Relationship to tonic and phasic REM sleep. Journal of Applied Physiology 48:5465. [ta]AH]

Orem, J., Montplaisir, J. \& Dement, W. C. (1974) Changes in the activity of respiratory neurons during sleep. Brain Research 82:309-15. [tajAH]

Oshima, T. (1983) Intracortical organization of arousal as a model of dynamic neuronal processes that may involve a set of movements. In: Motor control mechanisms in health and disease, ed. J. E. Desmedt. Raven Press. [MS]

Palkovits, M., Inke, G. \& Lukács, G. (1962) Topographische Beziehungen de menschlichen Subcommissuralorgans zur Epiphyse und ihre funktionelle Bedeutung. Endokrinologie 42:194-200. [JRN]

Palumbo, S. (1978) Dreaming and memory. Basic Books.

Pearlman, C. (1981) Rat models of the adaptive function of REM sleep. In: Slcep drcams and memory, ed. W. Fishbein. Spectrum Publications. [RG]

Panula, P., Yang, H. Y. T. \& Costa, E. (1984) Histamine-containing neurons in the rat hypothalamus. Procecdings of the National Academy Sciences (USA) 81:2572-76. [JMM]

Parker, I., Gundersen, C. B. \& Miledi, R. (1985) A transient inward current elicited by hyperpolarization during serotonin activation in Xenopus oocytes. Procecdings of the Royal Society (London) B223:279-92. [PAMvD]

Parmeggiani, P. L. (1981) Temperature regulation during sleep: A study in homeostasis. In: Physiology in sleep, ed. J. Orem \& C. D. Barnes. Academic Press. [taJAH]

Pasquier, D. A., Kemper, T. L., Forbes, W. B. \& Morgane, P. J. (1977) Dorsal raphe, substantia nigra and locus coeruleus: Interconnections with each other and the neostriatum. Brain Research Bulletin 2:323-39. [JMM]

Patton, H. D. (1965) Spinal reflexes and synaptic transmission. In: Neurophysiology, ed. T. C. Ruch, H. D. Patton, J. W. Woodburry \& A. L. Towe. Saunders. [PAMvD]

Pavel, S. (1978) Arginine vasotocin as a pineal hormone. Journal of Neural Transmission 13:135-55. [JRN]

(1979) The inechanism of action of vasotocin in the mammalian brain. Progress in Brain Rescarch 52:445-58. [JRN]

(1980) Presence of relatively high concentrations of arginine vasotocin in the cerebrospinal fuid of newborns and infants. Journal of Clinical Endocrinology and Mctabolism 50:271-73. [JRN]

Pavel, S. \& Eisner, C. (1984) A GABAergic habenulo-raphe pathway mediates both serotoninergic and hypnogenic effects of vasotocin in cats. Brain Rescarch Bulletin 13:623-27. [JRN]

Pavel, S. \& Coldstein, R. (1981) Narcoleptic-like alterations of sleep cycle in cats induced by specific vasotocin antiserum. Brain Research Bullctin 7:453-54. [JRN]

Pavel, S., Coldstein, R., Petrescu, M. \& Popa, M. (1981) REM sleep induction in prepubertal boys by vasotocin: Evidence for the involvement of serotonin containing neurons. Peptides 2:245-50. [JRN]

Pavel, S., Goldstein, R., Popoviciu, L., Corfariu, O., Foldes, A. \& Farkas, E. (1979) Pineal vasotocin: REM sleep dependent release into the cerebrospinal fluid of man. Waking and Sleeping 3:347-52. [JRN]

Pavel, S., Psatta, D. \& Goldstein, R. (1977) Slow-wave sleep induced in cats by extremely small amounts of synthetic and pineal vasotocin injected into the third ventricle of the brain. Brain Research Bulletin 2:251-54. [JRN]

Peterson, B. W. (1980) Participation of pontomedullary reticular neurones in specific motor activity. In: The reticular formation revisited, ed. J. A. Hobson \& M. A. B. Brazier. Raven Press. [taJAH]

Petitjean, F., Sakai, K., Blondaux, C. \& Jouvet, M. (1975) Hypersomnie par lésion isthmique chez le chat, 2 . Etude neurophysiologique et pharmacologique. Brain Research 88:439-53. [JMM]

Phillipson, E. A. (1978) Control of breathing during sleep. American Review of Respiratory Disease 118:909-39. [ta]AH]

Pickel, V. M., John, T. H. \& Reis, D. J. (1977) A serotonergic innervation of noradrenergic neurons in nucleus locus coeruleus: Demonstration by immunocytochemical localization of the transmitter specific enzymes tyrosine and tryptophan hydroxylase. Brain Research 131:197-214. [taJAH]

Pittendrigh, C. S. \& Daan, S. (1976) The functional analysis of circadian pacemakers in nocturnal rodents. Journal of Comparative Physiology 106:223-355. [rJAH]

Pivik, R. T., McCarley, R. W. \& Hobson, J. A. (1977) Eye movement associated discharge in brain stem neurons during desynchronized sleep. Brain Research 121:59-76. [ta]AH]

Polc, P., Schneeberger, J. \& Haefely, W. (1979) Effects of several centrally active drugs on the sleep-wakefulness cycle of cats. Ncuropharmacology 18:259-67. [WH]

Pompeiano, O. (1965) The neurophysiological mechanisms of the postural and motor events during desynchronized sleep. In: Association for Research in Nervous and Mental Disease, vol. 45, ed. S. K. Seymour, V. E. Edward \& L. W. Harold. Williams and Wilkins. [HK]

(1967) The neurophysiological mechanisms of the postural and motor events during desynchronized sleep. Proceedings of the Association of Research in Nervous and Mental Disease 45:351-423. [taJAH]

(1980) Cholinergic activation of reticular and vestibular mechanisms controlling posture and eye movements. In: The reticular formation revisited, ed. J. A. Hobson \& M. A. B. Brazier. Raven Press. [ta]AH]

Pompeiano, O. \& Hosino, K. (1976) Central control of posture: Reciprocal discharge by two pontine neuronal groups leading to suppression of decerebrate rigidity. Brain Research 116:131-38. [tarJAH]

Pompeiano, O. \& Valentinuzzi, M. (1976) A mathematical model for the mechanism of rapid eye movements induced by an anticholinesterase in the decerebrate cat. Archives Italiennes de Biologie 114:10354. [taJAH]

Popper, K. (1962) Conjectures and refutations. Basic Books. [taJAH] (1974) Replies to my critics. In: The philosophy of Karl Popper, Book 2, ed P. A. Schilpp. Open Court Publishers. [taJAH]

Prosser, R., Kittrell, E. M. W. \& Satinoff, E. (1984) Circadian body temperature rhythms in cats with suprachiasmatic nuclear lesions. In Thermal physiology, ed. J. R. S. Hales. Raven Press. [taJAH]

Puizillout, J. J., Gaudin-Chazal, G., Sayadi, A. \& Vigier, D. (1981) Serotoninergic mechanisms and sleep. Journal de Physiologic (Paris) 77:415-24. [JMM]

Ramón y Cajal, S. (1952) Histologic du système nerveux, vol. 1. Consejo Superior de Investigaciones Cientificas. [ta]AH]

Reppert, S. M., Artman, H. G., Swaminathan, S. \& Fisher, D. A. (1981) Vasopressin exhibits a rhythmic daily pattern in cerebrospinal fluid but not in blood. Science 213:1256-57. [tajAH]

Richter, C. P. (1965) Biological clocks in medicine and psychiatry. C. C. Thomas. [rJAH]

Riou, F., Cespuglio, R. \& Jouvet, M. (1982) Endogenous peptides and sleep in the rat. III. The hypnogenic properties of vasoactive intestinal polypeptide. Neuropeptides $2: 265-77$. [WF]

Roberts, M. H. T. \& Straughan, D. W. (1967) Excitation and depression of cortical neurons by 5-hydroxytryptamine. Journal of Physiology (London) 193:269-94. [CHV]

Robinson, T. E., Kramis, R. C. \& Vanderwolf, C. H. (1977) Two types of cerebral activation during active sleep: Relations to behavior. Brain Research 124:544-49. [CHV]

Roffwarg, H. (1979) Clossary of terms used in sleep disorders classification. Sleep 2:123-29. [ta]AH]

Roffwarg, H., Dement, W., Muzio, T. \& Fisher, C. (1962) Dream imagery Relationship to rapid eye movements. Archives of General Psychiatry 7:235-38. [RG] 
Roffwarg, H., Herman, J. \& Lamstein, S. (1975) The middle ear muscles: Predictability of their phasic activity in REM sleep and dream material. Sleep Research 4:165. [RG]

Roffwarg, H. P., Muzio, J. M. \& Dement, W. C. (1966) Ontogenetic development of the human sleep-dream cycle. Science 152:60419. [JRN]

Romo, R., Cheramy, A., Godehev, G. \& Glowinski, J. (1986) Physiological presynaptic facilitation of dopamine release in the cat candate nucleus. Journal of Physiology (Paris), in press. [rJAH]

Roney, K. J., Scheibel, A. B. \& Shaw, G. L. (1979) Dendrite bundles: Survey of anatomical experiments and physiological theories. Brain Research Reviews 1:225-71. [ABS]

Rønnekleiv, O. K., Kelly, M. J. \& Wuttke, W. (1980) Single unit recordings in the rat pineal gland: Evidence for habenulo-pineal neural connections. Experimental Brain Research 39:187-92. [JRN]

Rosenberg, P. A., Schweitzer, J. S. \& Dichter, M. C. (1985) Effects of norepinephrine on rat neocortical neurons in dissociated cell culture. Brain Research 344:369-72. [MS]

Rossi, G. F. \& Brodal, A. (1956) Corticofugal fibers to the brain stem reticular formation: An experimental study in the cat. Journal of Anatomy 90:42-62. [taJAH]

Ruch-Monachon, M. A., Jalfre, M. \& Haefely, W. (1976) Drugs and PGO waves in the lateral geniculate body of the curarized rat. I-V. Archives Internationales de Pharmacodynamia et de Therapie 219:251-346. [WH, PJM]

Ruch-Monachon, M. A., Jalfre, M. \& Haefely, W. (1976) Drugs and PGO waves in the lateral geniculate body of the curarized rat, 5. Synopsis of the role of central neurotransmitters on PGO wave activity. Archives Internationales de Pharmacoydnamie et de Thérapie 219:32646. [taJAH]

Saito, H., Sakai, K. \& Jouvet, M. (1977) Discharge patterns of the nucleus parabrachialis lateralis neurons of the cat during sleep and waking. Brain Research 134:59-72. [taJAH, MS]

Sakai, K. (1980) Some anatomical and physiological properties of pontomesencephalic tegmental neurons with special reference to the PGO waves and postural atonia during paradoxical sleep in the cat. In: The reticular formation revisited, ed. J. A. Hobson \& M. A. B. Brazier. Raven Press. [RC, taJAH, BEJ]

(1984) Central mechanisms of paradoxical sleep. In: Experimental brain research, Supplement 8, Sleep mechanisms, ed. A. Borbély \& J. L. Valatx. Springer. [taJAH]

(1985a) Anatomical and physiological basis of paradoxical sleep. In: Brain mechanisms of sleep, ed. D. J. McGinty, R. Drucker-Colin, A. Morrison \& L. Parmeggiani. Raven Press. [JMS, KS, MS, ZE]

(1985b) Neurons responsible for paradoxical sleep. In: Sleep: Neurotransmitters and neuromodulators, ed. A. Wauquier, J. M. Gaillard, J. M. Monti \& M. Radulovacki. Raven Press. [KS]

(1985c) Neurons responsible for paradoxical sleep. In: Sleep: Neurotransmitters and neuromodulators, ed. A. Wauguier, J. M. Manti, J. M. Gaillard \& M. Radulovacki. Raven Press. [tarJAH, BEJ]

Sakai, K., Kanamori, N. \& Jouvet, M. (1979) Activités unitaires spécifiques du sommeil paradoxal dans la formation réticulée bulbaires chez le chat nonrestreint. Comptes Rendues de l'Académie des Sciences, Série D 289:55761. $[\mathrm{KS}]$

Sakai, K., Salvert, D., Touret, M. \& Jouvet, M. (1977) Afferent connections of the nucleus raphe dorsalis in the cat as visualized by the horseradish peroxidase technique. Brain Research 137:11-35. [talAH, MS]

Sakai, K., Sastre, J.-P., Kanamori, N. \& Jouvet, M. (1981) State-specific neurons in the ponto-medullary reticular formation with special reference to the postural atonia during paradoxical sleep in the cat. In: Brain mechanisms and perceptual awareness, ed. O. Pompeiano \& $\mathrm{C}$. Ajmone Marsan. Raven Press. [taJAH]

Sakai, K., Sastre, J. P., Salvert, D., Touret, M., Tohyama, M. \& Jouvet, M. (1979) Tegmentoreticular projections with special reference to the muscular atonia during paradoxical sleep in the cat: An HRP study. Brain Research 176:233-54. [ta]AH]

Sakai, K., Touret, M., Salvert, D., Leger, L. \& Jouvet, M. (1977) Afferent projections to the cat locus coeruleus as visualized by the horseradish peroxidase technique. Brain Research 119:21-41. [taJAH]

Sakai, K., Vanni-Mercier, G. \& Jouvet, M. (1983) Evidence for the presence of PS-off neurons in the ventromedial oblongata of freely moving cats. Experimental Brain Research 49:311-14. [taJAH]

Sakakura, H. (1968) Spontaneous and evoked unitary activation of cat lateral geniculate neurons in sleep and wakefulness. Japanese Journal of Physiology 18:23-42. [ta]AH]

Sakamoto, T., Atsuta, Y. \& Mori, S. (1986) Long-lasting excitability changes of soleus alpha-motoneuron induced by midpontine stimulation in decerebrate, standing cat. Journal of Neurophysiology 55(3), in press. [SM]

Sallanon, M., Buda, C., Janin, M. \& Jouvet, M. (1985) Implication of serotonin in sleep mechanisms: Induction, facilitation? In: Slecp: Neurotransmitters and neuromodulators, ed. A. Wauquier, J. M. Monti, J. M. Gaillard \& M. Radulovacki. Raven Press. [JMM]

Sastre, J. P., Sakai, K. \& Jouvet, M. (1981) Are the gigantocellular tegmental field neurons responsible for paradoxical sleep? Brain Research 229:14761. [taJAH, WBM, JMS, PJS, RRV]

Satoh, K., Armstrong, D. M. \& Fibiger, H. C. (1983) A comparison of the distribution of central cholinergic neurons as demonstrated by acetylcholinesterase pharmacohistochemistry and choline acetyltransferase immunohistochemistry. Brain Research Bulletin 11:693-720. [BEJ]

Scheibel, M. E., Davies, T. \& Scheibel, A. B. (1973) Maturation of reticular dendrites: Loss of spines and development of bundles. Experimental Neurology 38:301-10. [ABS]

Scheibel, M. E. \& Scheibel, A. B. (1958) Structural substrates for integrative patterns in the brain stem reticular core. In: Reticular formation of the brain, ed. H. H. Jasper, L. D. Proctor, R. S. Knighton, W. S. Noshay \& R. T. Costello. Little, Brown. [taJAH, ABS]

(1973) The cellular basis of sleep cycle control. BIS Conference Report \#33. 14. [rJAH]

Scheibel, M. E., Tomiyasu, U. \& Scheibel, A. B. (1975) Do raphe nuclei of the reticular formation have a neurosecretory or vascular sensor function? Experimenial Neurology 47:316-29. [ABS]

Scherschlicht, R., Polc, P., Schneeberger, J., Steiner, M. \& Haefely, W. (1982) Selective suppression of rapid eye movement sleep (REMS) in cats by typical and atypical antidepressants. In: Typical and atypical antidepressants: Molecular mechanisms, ed. E. Costa \& G. Racagni. Raven Press. [WH]

Schulz, H., Dirlich, G., Balteskonis, S. \& Zulley, J. (1980) The REM-NREM sleep cycle: Renewal process or periodically driven process? Slecp 2:31928. [taJAH]

Segal, M. (1979) Serotonergic innervation of the locus coeruleus from the dorsal raphe and its action on responses to noxious stimuli. Journal of Physiology (London) 286:401-15. [taJAH]

Semba, J., Toru, M. \& Mataga, N. (1984) Twenty-four hour rhythms of norepinephrine and serotonin in nucleus suprachiasmaticus, raphe nuclei, and locus coeruleus in the rat. Sleep 7:211-18. [taJAH]

Shimazu, H. (1983) Neuronal organization of the premotor system controlling horizontal conjugate eye movements and vestibular nystagmus. In: Motor control mechanisms in health and disease, ed. J. E. Desmedt. Raven Press. [rJAH]

Shiromani, P. (1983) Long-term cholinergic stimulation of pontine nuclei: Effects on paradoxical sleep and memory. Ph.D. diss., City University of New York. [PJS]

Shiromani, P., Armstrong, D. M., Groves, P. M. \& Gillin, J. C. (1986) Combined neurophysiological and immunohistochemical mapping of the medial-lateral pontine reticular formation: Relationship of REM-on cells with respect to cells containing choline-acetyltransferase. Sleep Research 15 , in press. [PJS]

Shiromani, P., Barnett, J. \& Fishbein, W. (1982) Cholinergic stimulation of pontine cells by mini-pump infusion augments paradoxical sleep for fourteen days. Society for Neuroscience Abstracts 8:845. [WF]

Shiromani, P. \& Fishbein, W. (1980) Cholinergic stimulation of pontine brainstem cells by mini-pump infusion augments paradoxical sleep for five days. Society for Neuroscience Abstracts 6:708. [WF]

(1986) Continuous pontine cholinergic microinfusion via mini-pump induces sustained alterations in rapid eye movement sleep. Submitted for publication. [WF]

Shiromani, P. \& McGinty, D. J. (1983) Pontine sites for cholinergic PGO waves and atonia: Localization and blockade with scopolamine. Society for Neuroscience Abstracts 9:1203. [tajß H, PJS]

(1986) Pontine neuronal response to local cholinergic infusion: Relation to REM sleep. Brain Research, accepted for publication. [PJS]

Shiromani, P., Siegel, J. M. \& McGinty, D. J. (1984) Pontine carbachol microinfusions produce REM related decreases in blood pressure. Socicty for Neuroscience Abstracts 10:504. [ta]AH]

Shiromani, P., Siegel, J., Tomaszewski, K. \& McGinty, D. (1986) Alterations in blood pressure and REM sleep following pontine carbachol microinfusion. Experimental Neurology 91:285-92. [PJS]

Siegel, J. M. (1979a) Behavioral functions of the reticular formation. Brain Research Revicws 1:69-105. [PJS]

(1979b) Unit activity in the reticular formation and REM sleep. In: The functions of sleep, ed. R. Drucker-Colin, M. B. Sterman \& M. Shkurovich. Academic Press. [JMS]

Siegel, J. M. \& McGinty, D. J. (1977) Pontine reticular formation neurons: 
Relationship of discharge to motor activity. Science 196:678-80. [ta]AH, BEJ, JMS, PJS, WBM]

Siegel, J. M., McGinty, D. J. \& Breedlove, S. M. (1977) Sleep and waking activity of pontine gigantocellular field neurons. Experimental Neurology 56:533-73. [taJAH, RRV, JMS]

Siegel, J. M., Nienhuis, R. \& Tomaszewski, K. S. (1984) REM sleep signs rostral to chronic transections at the pontomedullary junction. Neuroscience Letters 45:241-46. [JMS]

Siegel, J. M. \& Tomaszewski, K. S. (1983) Behavioral organization of reticular formation: Studies in the unrestrained cat. I. Cells related to axial, limb, eye, and other movements. Journal of Neurophysiology 50:696-716. [JMS]

Siegel, J. M., Tomaszewski, K. S. \& Nienhuis, R. (1986) Behavioral states in the chronic medullary and mid-pontine cat. Electroencephalography and Clinical Ncurophysiology 63:279-88. [JMS]

Siegel, J. M., Tomaszewski, K. S. \& Wheeler, R. L. (1983) Behavioral organization of reticular formation: Studies in the unrestrained cat, 2. Cells related to facial movements. Journal of Neurophysiology 50:717-23. [JMS]

Silberman, E. K., Vivaldi, E., Gasfield, J., McCarley, R. W. \& Hobson, J. A. (1980) Carbachol triggering of desynchronized sleep via small volume infusions. Brain Rescarch 191:215-24. [KS]

Simon, R. P., Gershon, M. P. \& Brooks, D. C. (1973) The role of the raphe nuclei in the regulation of ponto-geniculo-occipital wave activity. Brain Rescarch 58:313-30. [taJAH]

Sitaram, N., Nurnberger, J. 1., Jr., Gershon, E. S. \& Gillin, J. C. (1982) Cholinergic regulation of mood and REM sleep: Potential model and marker of vulnerability to affective disorder. American Journal of Psychiatry 139:571-76. [taJAH]

Sitaram, N., Wyatt, R. J., Dawson, S. \& Gillin, J. C. (1976) REM sleep induction by physostigmine infusion during sleep. Science 191:128183. [taJAH]

Skagerberg, G., Björklund, A., Lindvall, O. \& Schmidt, R. H. (1982) Origin and termination of the diencephalo-spinal dopamine system in the rat. Brain Rescarch Bulletin 9:237-44. [JMM]

Sladek, J. R., Jr. (1971) Difference in the distribution of catecholamine varicosities in cat and rat reticular formation. Science 174:410-12. [tajAH]

Slozarska, M. \& Zernicki, B. (1973) Chronic pretrigeminal and cerveau isole cats. Acta Neurobiologiae Experimentalis 33:811-27. [H K]

Sofroniew, M. V. (1983) Morphology of vasopressin and oxytocin neurones and their central and vascular projections. Progress in Brain Research 60:101-14. [JRN]

Sofroniew, M. V., Priestley, J. V., Consolazione, A., Eckenstein, F. \& Cuello, A. C. (1985) Cholinergic projections from the midbrain and pons to the thalamus in the rat, identified by combined retrograde tracing and choline acetyltransferase immunohistochemistry. Brain Research 329:21323. [taJAH]

Speck, D. F. \& Feldman, J. C. (1982) The effects of microstimulation and microlesions in the ventral and dorsal respiratory groups in mcdulla of cat. Journal of Neuroscience 2:744-57. [ta]AH]

Sperry, R. W. (1950) Neural basis of the spontaneous optokinetic response produced by visual inversion. Journal of Comparative Physiology and Psychology 43:482-39. [ta]AH]

Sprague, J. M. (1966) Interaction of cortex and superior colliculus in mediation of visually guided behavior in the cat. Science 153:1544-47. [tajAH]

Steinfels, G. F., Heym, J., Strecker, R. E. \& Jacobs, B. L. (1983) Raphe unit activity in freely moving cats is altered by manipulations of central but not peripheral motor systems. Brain Research 279:77-84. [taJAH]

Steriade, M. (1978) Cortical long-axoned cells and putative interneurons during the sleep-waking cycle. Behavioral and Brain Sciences 3:465-514. [tarJAH]

(1981) Mechanisms underlying cortical activation: Neuronal organization and properties of the midbrain reticular core and intralaminar thalamic nuclei. In: Brain mechanisms and perceptual awareness, ed. O. Pompeiano \& C. Ajmone Marsan. Raven Press. [taJAH]

(1984) The excitatory-inhibitory response sequence in thalamic and neocortical cells: State related changes and regulatory systems. In: Dynamic aspects of neocortical function, ed. G. M. Edelman, W. E. Gall \& W. M. Cowan. Wiley. [taJAH]

Steriade, M. \& Deschenes, M. (1974) Inhibitory and interneuronal apparatus in motor cortex during sleep and waking, 2. Recurrent and afferent inhibition of pyramidal tract neurons. Journal of Neurophysiology 37:1093-1113. [ta]AH]

(1984) The thalamus as a neuronal oscillator. Brain Research Reviews 8:163. [tajAH]
Steriade, M., Deschenes, M. \& Oakson, G. (1974) Inhibitory processes and interneuronal apparatus in motor cortex during sleep and waking, 1. Background firing and responsiveness of pyramidal tract neurons and interneurons. Journal of Neurophysiology 37:1065-92. [taJAH]

Steriade, M. \& Glenn, L. L. (1982) Neocortical and caudate projections of intralaminar thalamic neurons and their synaptic excitation from midbrain reticular core. Journal of Neurophysiology 48:352-71. [taJAH]

Steriade, M. \& Hobson, J. A. (1976) Neuronal activity during the sleepwaking cycle. Progress in Neurobiology 6:153-376. [tarJAH]

Steriade, M., Iosif, G. \& Apostol, V. (1969) Responsiveness of thalamic and motor relays during arousal and various stages of sleep. Journal of Neurophysiology 32:251-65. [ta]AH]

Steriade, M., Kitsikis, A. \& Oakson, G. (1978) Thalamic inputs and subcortical targets of cortical neurons in areas 5 and 7 of cat. Experimental Neurology 60:420-42. [taJAH]

(1979) Excitatory-inhibitory processes in parietal association neurons during reticular activation and sleep-waking cycle. Sleep 1:339-55. [taJAH]

Steriade, M., Oakson, G. \& Ropert, N. (1982) Firing rates and patterns of midbrain reticular neurons during steady and transitional states of the sleep-waking cycle. Experimental Brain Research 46:37-51. [taJAH]

Steriade, M., Ropert, N., Kitsikis, A. \& Oakson, G. (1980) Ascending activation neuronal networks in midbrain reticular core and related rostral systems. In: The reticular formation revisited, ed. J. A. Hobson \& M. A. B. Brazier. Raven Press. [taJAH]

Steriade, M., Sakai, K. \& Jouvet, M. (1984) Bulbo-thalamic neurons related to thalamocortical activation processes during paradoxical sleep. Experimental Brain Research 54:463-75. [ta]AH, ZE]

Sterman, M. B., McGinty, D. J. \& Iwamura, Y. (1974) Modulation of trigeminal reflexes during the REM state in brain transected cats. Archives of Italian Biology 112:278-97. [JMS]

Stewart, D. J., MacFabe, D. F. \& Vanderwolf, C. H. (1984) Cholinergic activation of the electrocorticogram: Role of the substantia innominata and effects of atropine and quinuclidinyl benzilate. Brain Research 322:219-32. [CHV]

Strahlendorf, J. C., Strahlendorf, H. K., Kingsley, R. E., Gintautus, J. \& Barnes, C. D. (1980) Facilitation of the lumbar monosynaptic reflexes by locus coeruleus stimulation. Neuropharmacology 19:225-30. [rJAH]

Sun, Y.-a. \& Poo, M.-m. (1985) Non-quantal release of acetylcholine at a developing neuromuscular synapse in culture. Journal of Neuroscience 5:634-42. [ [JAH]

Swabb, D. F., Fliers, E., \& Partiman, T. S. (1985) The suprachiasmatic nucleus of the human brain in relation to sex, age and senile dementia. Brain Research 342:37-44. [WF]

Swanson, L. W. (1976) The locus coeruleus: A cytoarchitectonic Golgi and immunohistochemical study in the albino rat. Brain Research 110:39-56. [tajAH]

Szafarczyk, A., Ixart, G., Malaval, F., Nouguier-Soule, J. \& Assenmacher, I. (1979) Effects of lesions of the suprachiasmatic nuclei and of $p$ chlorophenylalanine on the circadian rhythms of adreno-corticotrophic hormone and corticosterone in the plasma, and on locomotor activity of rats. Journal of Endocrinology 83:1-16. [ta]A H]

Szymusiak, R. \& Satinoff, E. (1981) Maximal REM sleep time defines a narrower thermoneutral zone than does minimal metabolic rate. Physiology \& Behavior 26:687-90. [SD]

Taber-Pierce, E., Foote, W. E. \& Hobson, J. A. (1976) The efferent connection of the nucleus raphe dorsalis. Brain Research 107:13744. [taJAH]

Tobler, I. (1984) Evolution of sleep processes: A phylogenetic approach. In: Experimental brain research, Supplement 8, Slecp mechanisms, ed. A. Borbely \& J. L. Valatx. Springer. [ta]AH]

Tohyama, M., Sakai, K., Salvert, D., Touret, M. \& Jouvet, M. (1979) Spinal projections from the lower brain stem in the cat as demonstrated by the horseradish peroxidase technique, 1 . Origins of the reticulospinal tracts and their funicular trajectories. Brain Research 173:383-403. [tajAH]

Tohyama, M., Sakai, K., Touret, M., Salvert, D. \& Jouvet, M. (1979) Spinal projections from the lower brainstem in the cat as demonstrated by the horseradish peroxidase technique, 2. Projections from the dorsolateral pontine tegmentum and raphe nucleus. Brain Research 176:21531. [taJAH]

Trulson, M. E. \& Jacobs, B. L. (1979) Raphe unit activity in freely moving cats: Correlation with level of behavioral arousal. Brain Rescarch 163:135-50. [tajAH, JMM]

(1981) Activity of serotonin-containing neurons in freely moving cats. In: Serotonin neurotransmission and behavior, ed. B. L. Jacobs \& A. Gelperin. MIT Press. [CHV]

Trulson, M. E., Jacobs, B. L. \& Morrison, A. R. (1981) Raphe unit activity 
during REM sleep in normal cats and in pontine cats displaying REM sleep without atonia. Brain Research 226:75-91. [taJAH]

Trulson, M. E., Preussler, D. W. \& Howell, G. A. (1981) Activity of substantia nigra units across the sleep-waking cycle in freely moving cats. Neuroscience Letters 26:183-88. [JMM]

Ursin, R. \& Sterman, M. B. (1981) A manual for standardized scoring of sleep-waking states in the adult cat. Los Angeles: Brain Information Service, Brain Research Institute, University of California. [taJAH]

Valverdi, F. (1961) Reticular formation of the pons and medulla oblongata. A Golgi study. Journal of Comparative Neurology 116:71-99. [taJAH]

VanderMaelen, C. P. \& Aghajanian, G. K. (1980) Intracellular studies showing modulation of facial motoneurone excitability by serotonin. Nature 287:346-47. [PAMvD]

(1983) Electrophysiological and pharmacological characterization of serotonergic dorsal raphe neurons recorded extracellularly and intracellularly in rat brain slices. Brain Research 289:109-20. [PAMvD]

Vanderwolf, C. H. (1983) The role of the cerebral cortex and ascending activating systems in the control of behavior. In: Handbook of behavioral neurobiology, ed. E. Satinoff \& P. Teitelbaum. Plenum Press. [CHV]

(1984) Aminergic control of the electrocorticogram. In: Neurobiology of the trace amines, ed. A. A. Boulton, G. B. Baker, W. G. Dewhurst \& M. Sandler. Humana Press. [CHV]

Vanderwolf, C. H. \& Baker, G. B. (in press) Evidence that serotonin mediates non-cholinergic neocortical low voltage fast activity, noncholinergic hippocampal rhythmical slow activity and contributes to intelligent behavior. Brain Research. [CHV]

Vanderwolf, C. H. \& Robinson, T. E. (1981) Reticulo-cortical activity and behavior: $A$ critique of the arousal theory and a new synthesis. Behavioral and Brain Sciences 4:459-514. [CHV]

Van Dongen, P. A. M. (1981) The central noradrenergic transmission and the locus coeruleus: $A$ review of the data and their implications for neurotransmission and neuromodulation. Progress in Neurobiology 16:117-43. [PAMvD]

Van Dongen, P. A. M., Broekkamp, C. L. E. \& Cools, A. R. (1978) Atonia after carbachol microinjection near the locus coeruleus in cats. Pharmacology, Biochemistry and Behaviour 8:527-32. [PAMvD]

Van Dongen, P. A. M., Grillner, S. \& Hökfelt, T. (1986) 5Hydroxytryptamine (serotonin) causes a reduction in the afterhyperpolarization following the action potential in lamprey motoneurons and premotor interneurons. Brain Research, in press. [PAMvD]

Vertes, R. P. (1977) Selective firing of rat pontine gigantocellular neurons during movement and REM sleep. Brain Research 128:146-52. [taJAH, JMS, RRV]

(1979) Brain stem gigantocellular neurons: Patterns of activity during behavior and sleep in the freely moving rat. Journal of Neurophysiology 42:214-28. [RPV]

(1980) Brain stem activation of the hippocampus: A role for the magnocellular reticular formation and the MLF. Electroencephalography and Clinical Neurophysiology 50:48-58. [RPV]

(1981) An analysis of ascending brain stem systems involved in hippocampal synchronization and desynchronization. Journal of Neurophysiology 46:1140-59. [RPV]

(1982) Brain stem generation of the hippocampal EEG. Progress in Neurobiology 19:159-86. [RPV]

(1984) Brainstem control of the events of REM sleep. Progress in Neurobiology 22:241-88. [ZE, ta]AH, RRV]

Viala, D. \& Buser, P. (1969) The effect of DOPA and 5-HTP on rhythmic efferent discharges in hindlimb nerves in the rabbit. Brain Research 12:437-43. [rJAH]

Videen, T. O., Daw, N. W. \& Raider, R. K. (1984) The effect of norepinephrine on visual cortical neurons in kittens and adult cats. Journal of Neuroscience 4:1607-17. [rJAH]

Vijayashankar, N. \& Brody, H. (1979) Quantitative study of the pigmented neurons in the nuclei locus coeruleus and subcoeruleus in man as related to aging. Journal of Neuropathology and experimental Neurology 38:49097. [PAMvD]

Villablanca, J. (1962) Electroencephalogram in the permanently isolated forebrain of the cat. Science 138:44-46. [HK]

(1965) The electrocorticogram in the chronic cerveau isolé cat. Electroencephalography and Clinical Neurophysiology 19:576-86. [HK]

Vincent, S. R., Satoh, K., Armstrong, D. M. \& Fibiger, H. C. (1983) Substance $P$ in the ascending cholinergic reticular system. Nature 306:688-91. [ta]AH, MS]
Vivaldi, E. \& Hobson, J. A. (1981) Cholinergic REM sleep induction by the muscarinic agonist dioxolane. Sleep Research 10:104. [taJAH]

Vivaldi, E., McCarley, R. W. \& Hobson, J. A. (1980) Evocation of desynchronized sleep signs by chemical microstimulation of the pontine brainstem. In: The reticular formation revisited, ed. J. A. Hobson \& M. A. B. Brazier. Raven Press. [taJAH]

Vogel, G. W., Vogel, F., McAbee, R. S. \& Thurmond, A. J. (1980) Improvement of depression by REM sleep deprivation: New findings and a theory. Archives of General Psychiatry 37:247-53. [taJAH]

Walberg, F. (1974) Crossed reticulo-reticular projections in the medulla, pons and mesencephalon. Zeitschrift für Anatomie und Entwicklungsgeschichte 143:127-34. [tajAH]

Wamsley, J. K., Lewis, M. S., Young, W. S. \& Kuhar, M. J. (1981) Autoradiographic localization of muscarinic cholinergic receptors in rat brainstem. Journal of Neuroscience 1:176-91. [PJS]

Wang, R. Y. \& Aghajanian, G. K. (1977a) Physiological evidence for habenula as major link between forebrain and midbrain raphe. Science 197:8991. [JRN]

(1977b) Antidromically identified serotonergic neurons in the rat midbrain raphe: Evidence for collateral inhibition. Brain Research 132:18693. [taJAH]

Wang, R. Y., Gallager, D. W. \& Aghajanian, G. K. (1976) Stimulation of pontine reticular formation suppresses firing of serotonergic neurons in the dorsal raphe. Nature (London) 12:365-68. [taJAH]

Waterhouse, B. D., Moises, H. C. \& Woodward, D. J. (1981) Alpha-receptormediated facilitation of somatosensory cortical neuronal responses to excitatory synaptic inputs and iontophoretically applied acetylcholine. Neuropharmacology 20:907-20. [PAMvD]

Waterhouse, B. D., Moises, H. C., Yeh, H. H. \& Woodward, D. J. (1982) Norepinephrine enhancement of inhibitory synaptic mechanisms in cerebellum and cerebral cortex: Mediation by beta adrenergic receptors. Journal of Pharmacology and Experimental Therapeutics 221:495506. [PAMvD]

Webster, H. H., Friedman, L. \& Jones, B. E. (1986) Modification of paradoxical sleep following transections of the reticular formation at the pontomedullary junction. Sleep 9:1-23. [BEJ]

Wehr, T. A., Wirz-Justice, A., Goodwin, F. K., Duncan, W. \& Gillin, J. C. (1979) Phase advance of the circadian sleep-wake cycle as an antidepressant. Science 206:710-13. [taJAH]

Wever, R. A. (1984) Properties of human sleep-wake cycles: Parameters of internally synchronized free-running thythms. Sleep 7(1):27-51. [WF]

Wiesendanger, M. (1969) The pyramidal tract: Recent investigations on its morphology and function. Ergebnisse der Physiologie 61:73136. [taJAH]

Wilson, P. (1985) A photographic perspective on the origins, form, course and relations of the acetylcholinesterase-containing fibres of the dorsal tegmental pathway in the rat brain. Brain Research Reviews 10:85118. [PJS]

Wolf, N. J., Eckenstein, F. \& Butcher, L. L. (1984) Cholinergic systems in the rat brain, 1. Projections to the limbic telencephalon. Brain Research Reviews 13:751-84. [PJS]

Wood, J. D. \& Mayer, C. J. (1979) Serotonergic activation of tonic-type enteric neurons in guinea-pig small bowel. Journal of Neurophysiology 42:582-93. [PAMvD]

Yamaoka, S. (1980) Modification of circadian sleep rhythms by gonadal steroids and the neural mechanisms involved. Brain Research 185:38598. [WF]

Yezierski, R. P., Bowker, R. M., Kevetter, G. A., Westlund, K. N., Coulter, J. D. \& Willis, W. D. (1982) Serotonergic projections to the caudal brain stem: $A$ double label study using horseradish peroxidase and serotonin immunocytochemistry. Brain Research 239:258-64. [taJAH]

Zepelin, H. \& Rechtschaffen, A. (1974) Mammalian sleep, longevity, and energy metabolism. Brain, Behavior, and Evolution 10:425-70. [SD, rJAH]

Zigmond, R. E., Baldwin, C. \& Bowers, C. W. (1985) Rapid recovery of pineal function after partial denervation: A possible role for heteroneuronal uptake of transmitters in modulating synaptor efficacy. Joumal of Neuroscience 5:142-50. [rJAH]

Zulley, J. (1980) Distribution of REM sleep in entrained 24 hour and freerunning sleep-wake cycles. Sleep 2:377-89. [SD]

Zulley, J., Wever, R. \& Aschoff, J. (1981) The dependence of onset and duration of sleep on the circadian rhythm of rectal temperature. Pflugers Archives 391:314-18. [taJAH] 